DEUTSCHES ELEKTRONEN-SYNCHROTRON in der HELMHOLTZ-GEMEINSCHAFT

DESY-THESIS-2004-035

August 2004

General Search for New Phenomena in $e p$ Scattering at HERA

by

M. Wessels

ISSN $1435-8085$

NOTKESTRASSE 85 - 22607 HAMBURG 
DESY behält sich alle Rechte für den Fall der Schutzrechtserteilung und für die wirtschaftliche Verwertung der in diesem Bericht enthaltenen Informationen vor.

DESY reserves all rights for commercial use of information included in this report, especially in case of filing application for or grant of patents.

To be sure that your reports and preprints are promptly included in the HEP literature database send them to (if possible by air mail):

\begin{tabular}{||l|l||}
\hline DESY & DESY \\
Zentralbibliothek & Bibliothek \\
Notkestraße 85 & Platanenallee 6 \\
22607 Hamburg & 15738 Zeuthen \\
Germany & Germany \\
\hline
\end{tabular}




\title{
General Search for New Phenomena in $e p$ Scattering at HERA
}

\author{
Von der Fakultät für Mathematik, Informatik und Naturwissenschaften \\ der Rheinisch-Westfälischen Technischen Hochschule Aachen \\ zur Erlangung des akademischen Grades eines Doktors \\ der Naturwissenschaften genehmigte Dissertation
}

\author{
vorgelegt von \\ Diplom-Physiker \\ Martin Wessels \\ aus Hannover
}

Berichter: Universitätsprofessor Dr. Ch. Berger apl. Professor Dr. W. Braunschweig

Tag der mündlichen Prüfung: 29. Juli 2004

Diese Dissertation ist auf den Internetseiten der Hochschulbibliothek online verfügbar. 



\section{Abstract}

A model-independent search for deviations from the Standard Model prediction is performed in $e^{+} p$ and $e^{-} p$ collisions at HERA using H1 data corresponding to an integrated luminosity of $117 \mathrm{pb}^{-1}$. For the first time all event topologies involving isolated electrons, photons, muons, neutrinos and jets with high transverse momenta are investigated in a single analysis. Events are divided into exclusive event classes according to their final state. A novel statistical algorithm is used to search for deviations from the Standard Model in the distributions of the scalar sum of transverse momenta and invariant mass of final state particles and to quantify their significance. A good agreement with the Standard Model prediction is observed in most of the event classes and one interesting event is measured with four jets and an electron. The most significant deviation is found in a topology containing an isolated muon, missing transverse momentum and a jet, where a deviation has been previously reported.

\section{Kurzfassung}

In dieser Arbeit wird eine modellunabhängige Suche nach Abweichungen von der Vorhersage des Standardmodells in $e^{+} p$ und $e^{-} p$ Kollisionen bei HERA durchgeführt. Die analysierten H1-Daten entsprechen einer integrierten Luminosität von $117 \mathrm{pb}^{-1}$. Erstmals werden alle Ereignistopologien, die isolierte Elektronen, Photonen, Myonen, Neutrinos und Jets mit hohen Transversalimpulsen enthalten, in einer einzigen Analyse untersucht. Entsprechend ihres Endzustandes werden alle Ereignisse in exklusive Ereignisklassen unterteilt. Ein neuartiger statistischer Algorithmus wird benutzt, der in den Verteilungen der skalaren Summe der Transversalimpulse und der invarianten Masse nach Abweichungen vom Standardmodell sucht und deren Signifikanzen quantifiziert. In den meisten Ereignisklassen wird eine gute Übereinstimmung mit der Standardmodellvorhersage beobachtet, und ein interessantes Ereignis mit vier Jets und einem Elektron wird gemessen. Die signifikanteste Abweichung wird in einer Ereignistopologie mit einem isolierten Myon, fehlendem Transversalimpuls und einem Jet festgestellt, einer Topologie, in der schon in früheren Analysen Abweichungen beobachtet wurden. 



\section{Contents}

$\begin{array}{ll}\text { Introduction } & 1\end{array}$

1 Standard Model Physics at HERA 5

1.1 Inclusive Electron-Proton Scattering . . . . . . . . . . . . . 5

1.1 .1 Kinematics . . . . . . . . . . . . . . . . 6

1.1 .2 Cross Sections . . . . . . . . . . . . . . . . 7

1.1.3 Virtual Photon-Proton Scattering . . . . . . . . . . . . 9

1.1.4 Structure Functions in QPM and QCD . . . . . . . . . . . 11

1.2 Exclusive Final States . . . . . . . . . . . . . . . . . . 13

1.2 .1 Jet Production . . . . . . . . . . . . . . . . . . . . . 13

1.2.2 Photon Production . . . . . . . . . . . . . . . . . . 14

1.2.3 Lepton Pair Production . . . . . . . . . . . . . . . . . . . 18

$1.2 .4 \quad W$ Production . . . . . . . . . . . . . . . . . . 19

2 The H1-Experiment at HERA $\quad 21$

2.1 The Electron-Proton Accelerator HERA . . . . . . . . . . . . . . . . 21

2.2 The $\mathrm{H} 1$ Detector . . . . . . . . . . . . . . . . . . . . . . . . 24

2.2 .1 General Layout . . . . . . . . . . . . . . . . . . . . . 24

2.2.2 Inner Tracking System . . . . . . . . . . . . . . . . . . 26

2.2 .3 Calorimetry . . . . . . . . . . . . . . . . . . . . 29

2.2.4 Central Muon Detector . . . . . . . . . . . . . . . . . 31

2.2.5 Luminosity System . . . . . . . . . . . . . . . . . . . 32

2.3 Trigger and Data Acquisition . . . . . . . . . . . . . . . . . . . 33

2.4 Reconstruction of the Kinematics . . . . . . . . . . . . . . . . . 34

3 Monte Carlo Simulation $\quad 37$

3.1 Monte Carlo Generation . . . . . . . . . . . . . . . . . . . 37

3.2 Simulation . . . . . . . . . . . . . . . . . . . . . . 39

4 Data Selection and Classification $\quad 41$

4.1 Analysis Strategy . . . . . . . . . . . . . . . . . . . . . . 41

4.2 Event Selection . . . . . . . . . . . . . . . . . . . . . . . . 43

4.3 Particle Identification . . . . . . . . . . . . . . . . . . . . . . 44 
4.3 .1 Electrons . . . . . . . . . . . . . . . . . . . 45

4.3 .2 Photons . . . . . . . . . . . . . . . . . . . 54

4.3 .3 Muons . . . . . . . . . . . . . . . . . . . . . . . . . . 59

4.3 .4 Jets . . . . . . . . . . . . . . . . . . . 61

4.3 .5 Neutrinos . . . . . . . . . . . . . . . . . . . . 63

4.4 Event Classification . . . . . . . . . . . . . . . . . . . . . . . 64

$5 \quad$ Experimental Performance $\quad 67$

5.1 Energy Measurement and Calibration . . . . . . . . . . . . . . 67

5.1.1 Electromagnetic Energy Scale . . . . . . . . . . . . . . 67

5.1 .2 Hadronic Energy Scale . . . . . . . . . . . . . . . . 68

$5.2 \quad$ Trigger Efficiencies . . . . . . . . . . . . . . . . . 73

5.3 Limitations of the Measurement . . . . . . . . . . . . . . . 75

5.3.1 The $e-\nu$ Event Class . . . . . . . . . . . . . . . . . . . 75

5.3 .2 The $\mu-\nu$ Event Class . . . . . . . . . . . . . . . . . . . . . 77

5.4 Resolutions . . . . . . . . . . . . . . . . . . . . . 80

5.5 Purities and Efficiencies . . . . . . . . . . . . . . . . 82

5.6 Systematic Uncertainties . . . . . . . . . . . . . . . . . . . . . . . 84

5.7 Multi-Jet Topologies . . . . . . . . . . . . . . . . . . . . . . . 87

6 Experimental Results $\quad 91$

$7 \begin{array}{ll}\text { Search for Deviations } & 101\end{array}$

7.1 Search Algorithm . . . . . . . . . . . . . . . . . . . . . . . 101

7.2 Monte Carlo Experiments and Global Significance . . . . . . . . . . 105

7.3 Sensitivity to New Physics Signals . . . . . . . . . . . . . . . 107

7.4 Search Results . . . . . . . . . . . . . . . . . . . . . . . . 112

$\begin{array}{ll}\text { Summary } & 121\end{array}$

$\begin{array}{ll}\text { A Resolutions } & 123\end{array}$

$\begin{array}{ll}\text { B Purities and Efficiencies } & 127\end{array}$

$\begin{array}{ll}\text { C Systematic Uncertainties } & 131\end{array}$

D Event Display and Tables of the Results 133

$\begin{array}{ll}\text { List of Figures } & 137\end{array}$

$\begin{array}{ll}\text { List of Tables } & 141\end{array}$

$\begin{array}{ll}\text { Bibliography } & 143\end{array}$ 


\section{Introduction}

Today, the knowledge gained by scientists about the structure of matter is summarised in the Standard Model of particle physics, which has been developed in the second half of the last century and has proved very successfully in describing all experimentally results in the field of high energy physics. The basic assumptions of the Standard Model are simple and can be written down in a few lines.

Matter is composed of elementary fermions with spin $\frac{1}{2}$, quarks and leptons, each occurring in three families consisting of two particles. Each of the 12 particles has a corresponding anti-particle with opposite charge but otherwise identical properties.

$$
\text { leptons: }\left(\begin{array}{c}
\nu_{e} \\
e
\end{array}\right)\left(\begin{array}{c}
\nu_{\mu} \\
\mu
\end{array}\right)\left(\begin{array}{c}
\nu_{\tau} \\
\tau
\end{array}\right) \quad \text { quarks: }\left(\begin{array}{l}
u \\
d
\end{array}\right)\left(\begin{array}{l}
c \\
s
\end{array}\right)\left(\begin{array}{l}
t \\
b
\end{array}\right) \text {. }
$$

These elementary particles are subject to three fundamental forces, which are the strong, the electromagnetic and the weak force. Gravity, the forth known fundamental interaction, has not yet been included in the Standard Model. The interactions between the particles composing matter are mediated by bosons carrying spin 1 . These exchange particles are the massless photon for the electromagnetic force, the massive $Z^{0}$ and $W^{ \pm}$ bosons for the weak force and eight massless gluons mediating the strong interaction. The third kind of particle in the Standard Model is the Higgs boson, which is responsible for the creation of particle masses.

Towards a grand unification of the fundamental forces, the electromagnetic and weak force are combined in the Standard Model into the electroweak interaction. The gauge theory describing the strong interaction is the Quantum Chromodynamics.

The complex experiments nowadays arranged within the field of high energy physics to verify the predictions of the Standard Model are based on the same principle, which has already been utilised by Rutherford and his assistants Geiger and Marsden in their experiments revolutionising physics at the start of the 20th century. Aiming alpha particles on a thin gold foil, Rutherford deduced from the rate at which the alpha particles have been scattered at specific angles, that gold atoms must be largely made up of empty space containing a small heavy core, which carries nearly all of the mass and the total positive charge of the gold atom. The atomic nucleus had finally been discovered.

Since then our understanding of nature has improved, finally resulting in the conception of the Standard Model, and experimental technics have grown more complex. However, the basic principle of using point-like particles as a probe to investigate another particle 
that scatters the first has not changed for the recent experiments. Beneath these scattering experiments the investigation of electron-proton interactions has already played an important role in revealing the structure of the proton and establishing Quantum Chromodynamics as the theory of strong interactions. This successful story is continued at the $\mathrm{H} 1$ and ZEUS experiments at the Hadron-Elektron-Ring-Anlage HERA, which is the only accelerator colliding electrons on high-energetic protons worldwide.

At HERA electrons and protons collide with a centre-of-mass energy of up to $319 \mathrm{GeV}$. These high-energy electron-proton collisions provide a unique testing ground for the Standard Model. Although no deviation from the Standard Model has been established, it is widely believed that the Standard Model is incomplete and that new physics signals may appear below energies of $1 \mathrm{TeV}$. A large variety of extensions to the Standard Model has been constructed during the last decades, predicting various phenomena appearing at high energies or large transverse momenta. HERA data have been used to test some of these models of new processes by analysing their anticipated experimental signatures, and limits on their parameters have been derived [1].

Contrary to these dedicated analyses, the approach described in this thesis consists of a comprehensive and generic search for deviations from the Standard Model prediction at large transverse momenta $P_{T}$ in all final state topologies with at least two objects. For the first time all high- $P_{T}$ final state configurations involving electrons, muons, jets, photons or neutrinos are systematically investigated in a coherent analysis.

The presented analysis is based on the complete HERA-I data sample recorded at the H1 experiment between 1994 and 2000. All selected events are classified into exclusive event classes according to the number and types of objects detected in the final state. For all event classes the invariant mass and the scalar sum of transverse momenta distributions of the high $P_{T}$-final state objects are systematically researched for the appearance of new physics signals.

The analysis covers phase space regions where the Standard Model prediction is sufficiently precise to detect anomalies and does not rely on assumptions concerning the characteristics of any Standard Model extension. An in this spirit called model-independent search might therefore be able to discover unexpected manifestations of new physics. It addresses the important question of whether evidence of new physics might still be hidden in the data recorded at collider experiments.

The preliminary results of this analysis are reported in $[2,3]$. The prepared publication contains the result plots presented in this thesis.

In the first chapter of this thesis a theoretical overview of the scattering process of electrons on protons is given. In particular the production mechanism of the various final state topologies of the $e p$ interaction are discussed.

In the second chapter the HERA collider and the H1 experiment will be described. The third chapter introduces the Monte Carlo generators used in this analysis. Since a general search for deviations from the Standard Model requires a precise estimate of all 
relevant $e p$ scattering processes, multiple event generators are used.

The event selection and the quality criteria of the considered objects are described in chapter 4. Special emphasis will be placed on the electron and photon identification. Moreover the classification of events into exclusive event classes is introduced.

Chapter 5 is concerned with the performance of the experimental measurement. Both the electromagnetic and hadronic energy scale uncertainty are verified and the resolutions of the later on researched quantities are derived. Purities and efficiencies for each event class are determined and the systematic uncertainties considered are discussed. Finally, the feasibility of a measurement of multi-jet final states is investigated.

In chapter 6 the experimental results of the data measurement are introduced. The global event yields of the event classes are compared to the Standard Model expectation and the invariant mass and the scalar sum of transverse momenta distributions of the high- $P_{T}$ final state objects are discussed.

Finally in the seventh chapter, these distributions are systematically investigated within the framework of an statistical analysis using a novel algorithm, which locates the regions with the largest deviation between the data and the Standard Model prediction. In order to quantitatively determine the level of agreement between the data and the Standard Model expectation, the probability of occurrence of such a deviation is derived. 


\section{Chapter 1}

\section{Standard Model Physics at HERA}

At the Hadron-Elektron-Ring-Anlage HERA electrons ${ }^{1}$ and protons collide with a centreof-mass energy of up to $319 \mathrm{GeV}$. These high-energetic electron-proton interactions provide an unique testing ground for the Standard Model and allow for the investigation of complementary aspects compared to electron-positron annihilation and proton-antiproton scattering processes.

In the first part of this chapter the theoretical foundations on the inclusive scattering process of electrons and protons are introduced. Subsequently the production processes of exclusive final states are described. Most of the arguments presented in the following are derived from the discussions on electron-proton scattering as given in $[4,5,6,7,8,9]$.

\subsection{Inclusive Electron-Proton Scattering}

The investigation of electron-proton scattering processes has already played an important role in revealing the structure of the proton and in establishing Quantum Chromodynamics as the theory of the strong interaction between elementary particles.

At moderate energies the scattering of electrons on protons is essentially an elastic process, where the proton recoils from the impact but is actually resumed intact after the interaction. The differential cross section for the electron-proton elastic scattering has been calculated for the first time in 1950 and is known as Rosenbluth formula. It is dominated by so-called form factors, which determine how the scattering process is reduced from its value for a pointlike proton. In 1954 physicists supplied the experimental evidence that protons have a measurable diameter.

Raising the momentum transfer between the electron and proton and thus increasing the spatial resolution, the structure of the proton is resolved. Within this high-energetic probing process the proton is getting likely to break up, referred to as inelastic electron-proton scattering, where no restriction is applied on the type of the hadronic final state. In 1964 Gell-Mann and Zweig postulated that all hadrons are composed of pointlike particles, which Gell-Mann called quarks. From that point of view the elastic electron-quark scat-

\footnotetext{
${ }^{1}$ In this thesis "electron" refers to both electrons and positrons, if otherwise not stated.
} 

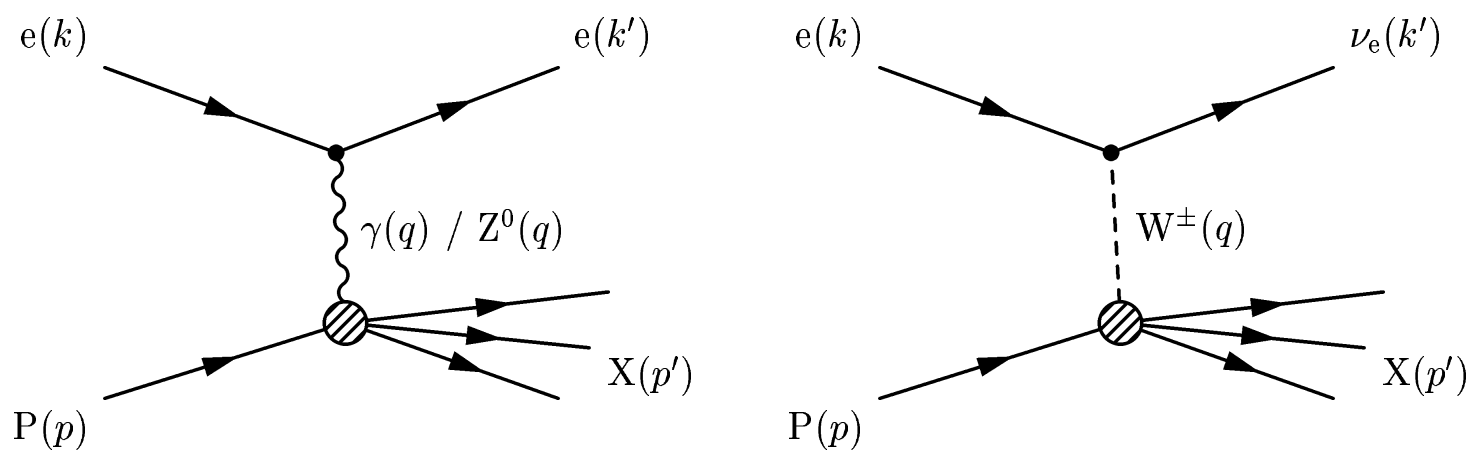

Fig. 1.1: The lowest-order diagrams of electron-proton scattering for neutral current processes (left) and charged current processes (right). The four-momenta of the particles or particle systems are given in parentheses.

tering is getting accessible within the inelastic scattering process of electrons on protons. Finally, the early 1970's saw the discovery of the quarks in experiments performed at the Stanford Linear Accelerator SLAC.

\subsubsection{Kinematics}

Within the Standard Model of particle physics the theoretical description of the scattering process of electrons on protons is based on the theory of electroweak interactions and the Quantum Chromodynamics. In the most general case the amount of possible reactions is subdivided into two kinds of processes according to the type of the intermediate bosons as depicted in figure 1.1.

The incoming electron with the four-momentum $k$ interacts with the proton carrying the four-momentum $p$ via the exchange of an electroweak gauge boson transferring the fourmomentum $q$. Since the lepton number has to be conserved, the final state consist of a scattered lepton with the four-momentum $k^{\prime}$, while the struck proton might fragment into the hadronic system $X$ carrying the four-momentum $p^{\prime}$. In case of neutral current (NC) processes $e p \rightarrow e X$ the exchanged boson is a neutral particle $\left(\gamma\right.$ or $\left.Z^{0}\right)$, while charged particles $\left(W^{+}\right.$or $\left.W^{-}\right)$mediate the interaction in charged current (CC) processes $e p \rightarrow \nu X$ resulting in the outgoing lepton being a neutrino.

Based on the four-momenta of the initial electron, the final state lepton and the incoming proton the kinematics of the electron-proton scattering are determined at the lowest-order by the following three Lorentz-invariant variables:

$$
\begin{aligned}
Q^{2} & =-q^{2}=-\left(k-k^{\prime}\right)^{2}, \\
x & =\frac{Q^{2}}{2(p \cdot q)}, \\
y & =\frac{p \cdot q}{p \cdot k} .
\end{aligned}
$$


$Q^{2}$ defined as the negative square of the four-momentum transfer $q$ is a measure of the virtuality of the intermediate boson and determines the hardness or resolving power of the interaction. The non-dimensional variables $x$ and $y$ are ranging between zero and one and are best understood in dedicated frames of reference. $x$ is called the Bjørken scaling variable. Its value evaluated in the infinite momentum frame of the proton can be interpreted in the framework of the Quark Parton Model as the proton momentum fraction carried be the struck quark (cf. section 1.1.4). In the rest frame of the proton $y$ determines the relative energy loss of the scattered electron and gives thus a measure of the inelasticity of the interaction ${ }^{2}$.

The square of the centre-of-mass energy $s$ and the square of the invariant mass $W^{2}$ of the hadronic system $X$ recoiling against the scattered lepton are given by

$$
s=(k+p)^{2} \quad \text { and } \quad W^{2}=(p+q)^{2}=p^{2} .
$$

Based on the invariant mass $W$ of the hadronic system the mentioned transition from the elastic to the inelastic regime of electron-proton scattering can be formulated more quantitatively. In case of elastic interactions where the proton does not break up the value of $W$ apparently amounts to the mass of the proton. For increasing energy transfers the proton might be excited into a resonant state, such as the $\Delta^{+}$. In this case of the socalled quasi-elastic scattering the invariant mass $W$ reaches values in the order of the mass of the according resonance. Entering the regime of inelastic electron-proton scattering, the complicated multi-particle final states with large invariant masses result in a smooth distribution of $W$.

Neglecting the electron and proton masses the square of the centre-of-mass energy is connected with the energies of the incoming electron $E_{k}$ and proton $E_{p}$ via the relation

$$
s=4 E_{k} E_{p}
$$

Under the same assumption the following equations are obtained, relating the introduced kinematic variables $Q^{2}, x, y, W^{2}$ and $s$ :

$$
Q^{2}=\operatorname{sxy} \quad W^{2}=Q^{2} \frac{1-x}{x} .
$$

Thus in case of fixed centre-of-mass energies $\sqrt{s}$, as it is true for the HERA accelerator, only two of those four Lorentz-invariant variables $Q^{2}, x, y, W^{2}$ are independent and sufficient to determine the kinematics of the electron-proton scattering.

\subsubsection{Cross Sections}

As discussed in the previous section the inclusive differential cross section, integrated over all possible hadronic final states of the electron-proton scattering process, is a function

\footnotetext{
${ }^{2}$ In the proton rest frame $y$ is given by $y=1-\frac{E_{k^{\prime}}}{E_{k}}$, where $E_{k}$ and $E_{k^{\prime}}$ denote the energies of the incoming electron and scattered lepton in this frame of reference.
} 
of two independent variables uniquely determining the kinematics of the events. In anticipation of the structure of the proton the variables $x$ and $Q^{2}$ are typically chosen for parameterisation.

Based on the diagrams presented in figure 1.1 the double differential cross section for inelastic electron-proton scattering is given at lowest order in electroweak interactions in the most general formulation by the convolution of a leptonic tensor $L_{\mu \nu}$ and a hadronic tensor $W^{\mu \nu}$, which describe the currents at the corresponding vertices [5]:

$$
\frac{d^{2} \sigma}{d x d Q^{2}} \propto \epsilon_{V} L_{\mu \nu}^{V}(k, q) W_{V}^{\mu \nu}(p, q)
$$

The subscript $V$ labels the exchanged vector bosons $\gamma$ and $Z^{0}$ for neutral current processes and $W^{ \pm}$in case of charged current interactions. The according couplings of the intermediate bosons to the particles involved in the scattering process are given by $\epsilon_{V}$. Whereas the leptonic part of the diagrams is calculable within the electroweak theory the tensor $W_{V}^{\mu \nu}$ serves to parametrise the ignorance of the form of the currents at the hadronic vertex. Exploiting the Lorentz structure of the hadronic tensor and the restriction of current conservation the double differential cross section for unpolarised electron-proton scattering can finally be expressed as [4]

$$
\frac{d^{2} \sigma^{e^{ \pm} p}}{d x d Q^{2}}=\mathcal{A}\left[\frac{y^{2}}{2} 2 x \mathcal{F}_{1}\left(x, Q^{2}\right)+(1-y) \mathcal{F}_{2}\left(x, Q^{2}\right) \mp\left(y-\frac{y^{2}}{2}\right) x \mathcal{F}_{3}\left(x, Q^{2}\right)\right] .
$$

The factor $\mathcal{A}$ varies for neutral and charged current processes and is given by

$$
\mathcal{A}^{N C}=\frac{4 \pi \alpha^{2}}{x Q^{4}} \quad \text { and } \quad \mathcal{A}^{C C}=\frac{G_{F}^{2}}{2 \pi x}\left(\frac{M_{W}^{2}}{Q^{2}+M_{W}^{2}}\right)^{2},
$$

where $M_{W}$ denotes the mass of the $W$ boson and $G_{F}$ is the Fermi constant.

The unknown interaction at the proton side is absorbed in the definition of the generalised proton structure functions $\mathcal{F}_{i}\left(x, Q^{2}\right)$, which are sensitive to the structure of the proton and are in general functions of the kinematics of the scattering process [10]. The generalised structure functions are process dependent, since they encode the contributions arising from the exchange of the various electroweak bosons. In such a way the expected $Z^{0}$ propagator is completely absorbed in case of neutral current interactions. The structure function $\mathcal{F}_{3}$ is non-zero only for weak interactions as it is generated by the parity violating interactions.

Typically, the longitudinal structure function $\mathcal{F}_{L}$ is introduced as

$$
\mathcal{F}_{L}\left(x, Q^{2}\right)=\mathcal{F}_{2}\left(x, Q^{2}\right)-2 x \mathcal{F}_{1}\left(x, Q^{2}\right)
$$

and the double differential cross section for the inelastic electron-proton scattering is expressed in terms of the structure functions $\mathcal{F}_{2}, \mathcal{F}_{3}$ and $\mathcal{F}_{L}$. 
The dependence of the inelastic cross sections on $Q^{2}$ is principally formed by the propagator terms, which are given by $1 / Q^{4}$ for the neutral current process and $1 /\left(Q^{2}+M_{W}^{2}\right)^{2}$ for charged current scattering. For neutral current interactions the contribution arising from the exchange of $Z^{0}$ bosons becomes important at $Q^{2} \gtrsim M_{Z}^{2}$ with $M_{Z}$ denoting the mass of the $Z^{0}$ boson.

At low values of $Q^{2}$ the electron-proton interaction is dominated by the neutral current scattering process, whose cross section is about three orders of magnitude larger than that of charged current interactions in this kinematic region. But while the neutral current cross section decreases very rapidly with increasing values of $Q^{2}$ the charged current cross section falls much less steeply up to values of $Q^{2}$ in the order of $M_{W}^{2}$. In this kinematic regime, which is in fact the regime of electroweak unification, the charged current cross section measures up to the cross section of neutral current scattering and the characteristics of both cross sections become similar. For reasons becoming apparent in section 1.1.4 the electron-proton scattering at HERA is referred to as deep-inelastic in the kinematic region of $Q^{2} \gtrsim 4 \mathrm{GeV}^{2}$.

The electron-proton scattering is dominated by processes with very small momentum transfers. In this kinematic domain the weak interactions mediated by $Z^{0}$ and $W^{ \pm}$exchange can be neglected compared to the dominant cross section of the electromagnetic scattering process. In the limit $Q^{2} \rightarrow 0 \mathrm{GeV}^{2}$ the exchanged photon adopts the character of real photons and the electron-proton interaction can be considered as photon-proton scattering. The electron-proton scattering is then referred to as photoproduction, in analogy to the denotation of the process of hadron production in the scattering of real photons on nucleons $\gamma+N \rightarrow X$.

The following section describes the approach of virtual photon-proton scattering which allows for the transition from the regime of deep-inelastic electron-proton scattering to the region of photoproduction.

\subsubsection{Virtual Photon-Proton Scattering}

Apart from the approach presented in section 1.1.2 the scattering process of electrons on protons can also be described as the convolution of a flux of virtual bosons radiated from the electron with the absorption cross section of a virtual boson by the proton. In the following discussion only the dominant electromagnetic process, the scattering of virtual photons on protons, is considered:

$$
\gamma^{*}(q)+P(p) \rightarrow X\left(p^{\prime}\right)
$$

The virtual photon is treated as a massive spin 1 particle. Thus the cross section of the electron-proton scattering is split into two independent parts, one for the absorption of transversely polarised photons $\sigma_{T}$ and one for longitudinally polarised photons $\sigma_{L}[6]$ :

$$
\begin{aligned}
\frac{d^{2} \sigma_{N C}}{d y d Q^{2}} & =\Gamma_{T} \sigma_{T}\left(Q^{2}, W^{2}\right)+\Gamma_{L} \sigma_{L}\left(Q^{2}, W^{2}\right) \\
& =\Gamma_{T}\left[\sigma_{T}\left(Q^{2}, W^{2}\right)+\epsilon \sigma_{L}\left(Q^{2}, W^{2}\right)\right]=\Gamma_{T} \sigma_{\gamma^{*} p}^{e f f}\left(y, Q^{2}, W^{2}\right) .
\end{aligned}
$$


The functions $\Gamma_{T}$ and $\Gamma_{L}$ denote the according fluxes of transversely and longitudinally polarised photons and are given by

$$
\begin{gathered}
\Gamma_{T}\left(y, Q^{2}\right)=\frac{1}{\epsilon(y)} \Gamma_{L}\left(y, Q^{2}\right)=\frac{\alpha}{2 \pi Q^{2} y}\left(1+(1-y)^{2}\right) \\
\text { with } \epsilon(y)=\frac{2(1-y)}{1+(1-y)^{2}} .
\end{gathered}
$$

The photon polarisation $\epsilon=\Gamma_{T} / \Gamma_{L}$ is given by the ratio of the longitudinal to the transverse photon flux and $\sigma_{\gamma^{*} p}^{e f f}=\sigma_{T}+\epsilon \sigma_{L}$ is known as the effective $\gamma^{*} p$ cross section.

The structure functions of the proton $\mathcal{F}_{1}, \mathcal{F}_{2}$ and $\mathcal{F}_{3}$ are connected with the absorption cross sections $\sigma_{T}$ and $\sigma_{L}$ by the following relations:

$$
2 x \mathcal{F}_{1}=\frac{Q^{2}}{4 \pi^{2} \alpha} \sigma_{T} \quad \mathcal{F}_{2}=\frac{Q^{2}}{4 \pi^{2} \alpha}\left(\sigma_{T}+\sigma_{L}\right) \quad \mathcal{F}_{L}=\frac{Q^{2}}{4 \pi^{2} \alpha} \sigma_{L}
$$

The total virtual photon-proton cross section $\sigma_{\gamma^{*} p}^{t o t}$ is defined by the sum of the transverse and longitudinal absorption cross sections and is given by

$$
\sigma_{\gamma^{*} p}^{\text {tot }}=\sigma_{T}\left(Q^{2}, W^{2}\right)+\sigma_{L}\left(Q^{2}, W^{2}\right)=\frac{4 \pi^{2} \alpha}{Q^{2}} \mathcal{F}_{2}\left(Q^{2}, W^{2}\right)
$$

Contrary to the effective $\gamma^{*} p$ cross section $\sigma_{\gamma^{*} p}^{\text {eff }}$ the total cross section as derived above depends only on the variables $Q^{2}$ and $W^{2}$.

Within the approach of virtual photon-proton scattering the cross section for the electronproton inelastic scattering in the kinematic regime of photoproduction at $Q^{2} \approx 0 \mathrm{GeV}^{2}$ can be derived. In the limit $Q^{2} \rightarrow 0$ the longitudinal component of the electron-proton cross section vanishes, since the mediated photon adopts the character of real photons, which are only transversely polarised. The transverse absorption cross section $\sigma_{T}\left(Q^{2}, W^{2}\right)$ converges to the photoproduction cross section $\sigma_{\gamma p}\left(W^{2}\right)$. The differential electron-proton scattering cross section as a function of $y$ is given within this approach by

$$
\frac{d \sigma}{d y}=f_{\gamma / e} \sigma_{\gamma p}
$$

The flux of the photons $f_{\gamma / e}$ as derived in the Weizsäcker-Williams approximation $[11,12]$ is obtained by integrating (1.11) over $Q^{2}$ :

$$
f_{\gamma / e}\left(y, Q_{\max }^{2}\right)=\frac{\alpha}{2 \pi y}\left[\left(1+(1-y)^{2}\right) \ln \frac{(1-y) Q_{\max }^{2}}{m_{e}^{2} y^{2}}-2(1-y)\right],
$$

where $m_{e}$ denotes the mass of the electron and the value of $Q_{\max }^{2}$ is determined by the maximal angle allowed for the scattered electron. Compared to the integral of relation (1.13) the additional term $2(1-y)$ within the obtained expression for the photon flux arises from the consideration of terms in the order of $m_{e}$. 


\subsubsection{Structure Functions in QPM and QCD}

Already in the 1960's the proton was assumed to be composed of a small number of pointlike constituents, the so-called partons, which have later on be identified with quarks and gluons. Since the structure functions $\mathcal{F}_{i}$ parameterise the proton structure, they are suited to explore the properties of the partons. The physical interpretation of the structure functions within the Quark Parton Model (QPM) and the impact of the Quantum Chromodynamics (QCD) are presented in the following.

In the late 1960's Bjørken predicted that in the regime of high energies the proton structure functions are independent of the transfered momentum $Q^{2}$ and functions of the kinematic variable $x$ alone ${ }^{3}$ :

$$
\mathcal{F}_{i}\left(x, Q^{2}\right) \rightarrow \mathcal{F}_{i}(x) \quad \text { Bjørken scaling . }
$$

In 1969 Callan and Gross proposed that in case of scaling structure functions the longitudinal structure function $\mathcal{F}_{L}$ would vanish, resulting from the following relation between $\mathcal{F}_{1}(x)$ and $\mathcal{F}_{2}(x)$ :

$$
\mathcal{F}_{L}=0 \Leftarrow 2 x \mathcal{F}_{1}(x)=\mathcal{F}_{2}(x) \quad \text { Callan-Gross relation } .
$$

Both predictions have impressively been confirmed by experiments at the SLAC in the early 1970's and result directly from the interpretation of the electron-proton scattering within the Quark Parton Model, which was proposed by Feynman in 1969.

\section{Quark Parton Model}

In a reference frame where the proton momentum is large (infinite momentum frame) the partons composing the proton have only a small transverse momentum. Thus the proton is described by a beam of collinear partons, each carrying a certain fraction of the total proton momentum.

In the Quark Parton Model the proton is viewed to be composed of three pointlike partons with spin $\frac{1}{2}$, identified with two up-quarks and one down-quark. The electron-proton interaction is expressed as the incoherent scattering of the electron on free quarks inside the proton, each carrying the momentum fraction $x$.

Introducing the quark momentum distributions $q(x)$ the proton structure functions $\mathcal{F}_{i}$ are given within this approach by the following relations [4]:

$$
\begin{aligned}
& \mathcal{F}_{1}=\frac{1}{2} \sum_{f} q_{f}(x)\left(v_{f}^{2}+a_{f}^{2}\right), \\
& \mathcal{F}_{2}=\sum_{f} x q_{f}(x)\left(v_{f}^{2}+a_{f}^{2}\right), \\
& \mathcal{F}_{3}=2 \sum_{f} q_{f}(x) v_{f} a_{f},
\end{aligned}
$$

\footnotetext{
${ }^{3}$ The so-called Bjørken-Limit denotes the transition $Q^{2} \rightarrow \infty$ and $p \cdot q \rightarrow \infty$ with fixed values for $x=Q^{2} /(2 p \cdot q)$. In this kinematic regime the electron-proton scattering is referred to as deep-inelastic.
} 
where the sum runs over all quark and antiquark flavours $f$ which participate in the interaction. Depending on the underlying process the according vector and axial-vector couplings of the electroweak gauge bosons on the quarks are given by $v_{f}$ and $a_{f}$, respectively.

For neutral current processes mediated by photons only, the couplings $v_{f}$ amount to the charges of the quarks $e_{f}$ while the axial-vector couplings $a_{f}$ equal to zero. In case of charged current interactions the vector and axial-vector couplings are given by $v_{f}=a_{f}=1$ for quarks and $v_{f}=-a_{f}=1$ for antiquarks. The couplings have a more complicated structure for neutral current interactions involving the $Z^{0}$ exchange and its interference with the photon contribution. The explicit expressions for the structure functions in those cases are given for instance in [13].

Apparently, the Bjørken scaling is realised within the Quark Parton Model, since no dependence of the structure functions on the momentum transfer $Q^{2}$ is derived. This behaviour results from the assumption, that the proton is composed of pointlike but non-interacting constituents. The completion of the Callan-Gross relation, which is also directly deducible from the given relations for the structure functions, is a direct consequence of the considerations of charged partons with spin $\frac{1}{2}$.

\section{The Impact of Quantum Chromodynamics}

Both the Bjørken scaling and Callan-Gross relation are violated when leaving the naive approach of the Quark Parton Model and taking into account the strong interactions between the quarks as described by the Quantum Chromodynamics.

Within the description of QCD the quarks are bound in the proton due to interactions mediated by gluons, involving processes such as gluon radiation $(q \rightarrow q g)$ and the conversion of gluons into quark-antiquark pairs $(g \rightarrow q \bar{q})$. The emission and reabsorption of gluons by the quarks modifies the transverse quark momenta and allows the quarks to couple on longitudinally polarised bosons. Thus the contribution of the longitudinal absorption cross section $\sigma_{L}$ (cf. section 1.1.3) can not be neglected any longer, resulting in the violation of the Callan-Gross relation.

In addition the radiation of gluons results in a logarithmic dependence of the structure functions on $Q^{2}$, representing the violation of Bjørken scaling. As a consequence of the conversion of gluons into quark-antiquark pairs, which themselves may radiate gluons creating further quark-antiquark pairs and so on, the proton content is modified. Contrary to the approach of the QPM the proton exists no longer only of three valence quarks, but contains also a fraction of gluons and sea-quarks, which carry small momentum fractions $x$. Depending on the momentum of the probing gauge boson this partonic structure of the proton is resolved. 


\subsection{Exclusive Final States}

The previous chapter has concentrated on the inelastic scattering process of electrons on protons abstracting away from the composition of the generated final state. The measurements of the inclusive cross sections give directly access to the proton structure functions, which reveal the nature of the partons and allow in particular for the investigation of Quantum Chromodynamics.

Anyhow, the pattern of perturbative QCD is maybe even better accessible in the exploration of the hadronic final state. Although coloured partons cannot be observed directly, their fragmentation produces jets of hadrons collimated around the primal direction of the partons. In such a way the gluon has been directly observed for the first time in the measurement of three-jet events at the PETRA accelerator at DESY in 1979.

The investigation of exclusive final states allows for the search for signals of new physics which would manifest themselves in dedicated event topologies. Moreover, the variety of final state topologies reflects the physics of elementary particles at large and their exploration allows therewith for a general verification of the Standard Model.

The following paragraphs introduce the theoretical foundations on the formation of exclusive final states in electron-proton scattering at HERA.

\subsubsection{Jet Production}

In case of the electron-proton scattering being inelastic, the proton is breaking up within the probing process and the final state is expected to include jets of hadronic particles arising from the partons composing the proton.

At the lowest order of the strong coupling constant $\alpha_{s}$, which is actually $\mathcal{O}\left(\alpha_{s}^{0}\right)$, the final state of a typical deep-inelastic electron-proton interaction consist of a jet of hadrons originating from the struck quark, called the current or leading jet, which balances the transverse momentum of the scattered lepton. The remnant of the proton also fragments into hadrons, which remain in the centre-of-mass frame collimated around the primal direction of the incoming proton. Thus the hadronic final state of events of this kind is referred to as being composed of $(1+1)$ jets. The corresponding diagram is shown in figure 1.2 (a) for the dominant electromagnetic ep scattering. The diagrams for processes involving the exchange of $Z^{0}$ or $W^{ \pm}$bosons look similar.

At the first order of $\alpha_{s}(2+1)$ jet final states are produced. A gluon may split into a pair of quarks with large relative transverse momenta, before one of the quarks absorbs the virtual boson. This processes is known as Boson-Gluon fusion and shown in figure 1.2 (b). Another process resulting in two high transverse momentum jets is the so-called QCD Compton scattering, in which the quark radiates a hard gluon (before) absorbing the virtual boson as shown in figure 1.2 (c). Even higher jet multiplicities are produced taking into account further QCD radiation effects.

The production of jets in the inelastic electron-proton scattering is dominated by photoproduction processes. As discussed in section 1.1.3 the incoming electron can be regarded 


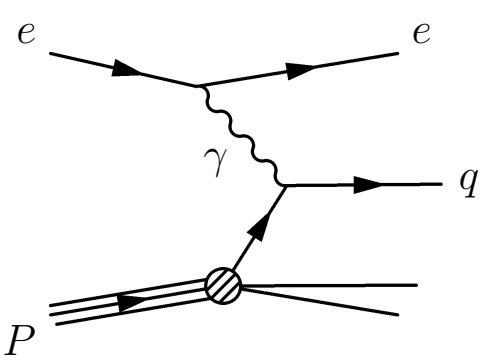

(a)

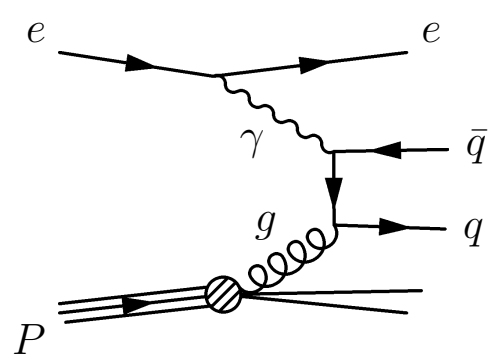

(b)

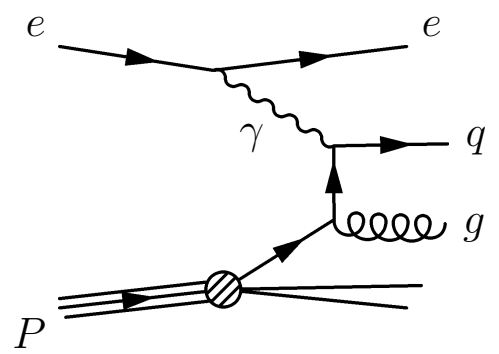

(c)

Fig. 1.2: Diagrams for jet production at lowest (a) and first order (b-c) of the strong coupling constant $\alpha_{s}$ for the inelastic electromagnetic ep scattering. The processes displayed in figure (b) and (c) are known as Boson-Gluon fusion and QCD Compton scattering, respectively. For both processes only the $t$-channel contribution is shown.

as a source of photons, which are favoured to have quasi-real character due to the characteristics of the photon propagator. Resulting from the conservation of four-momentum the production of $(1+1)$ jet final states as displayed in figure 1.2 (a) is not realised in the regime of photoproduction for photons on the mass shell. As a further consequence of its low virtuality the photon may fluctuate before the interaction into quark pairs, resulting in a partonic structure of the photon.

Consequently the photoproduction of jets is described either by the direct scattering of a real photon on a parton of the proton (direct photoproduction) or the interaction between one of the partons inside the photon with one of the partons of the proton (resolved photoproduction). Apart from the virtuality of the photon, the direct photoproduction of jets is in the same way as for the deep-inelastic regime in leading order of $\alpha_{s}$ described by the diagrams presented in figure 1.2 (b) and (c). Although the hard subprocess of the resolved photoproduction of $(2+1)$ jets is in the order of $\alpha_{s}^{2}$ the impact of the parton density function of the photon provokes both processes to contribute roughly in the same order of magnitude in the regime of high transverse momenta. The leading-order diagrams for the resolved photoproduction of jets are displayed in figure 1.3.

\subsubsection{Photon Production}

Photons in the final state of the electron-proton interaction arise either from direct production processes or from the fragmentation process of the partons. In the latter case the photons are typically included in hadronic jets and thus in general not identifiable as isolated particles. The following paragraphs describe the production of hard photons in electron-proton scattering. 

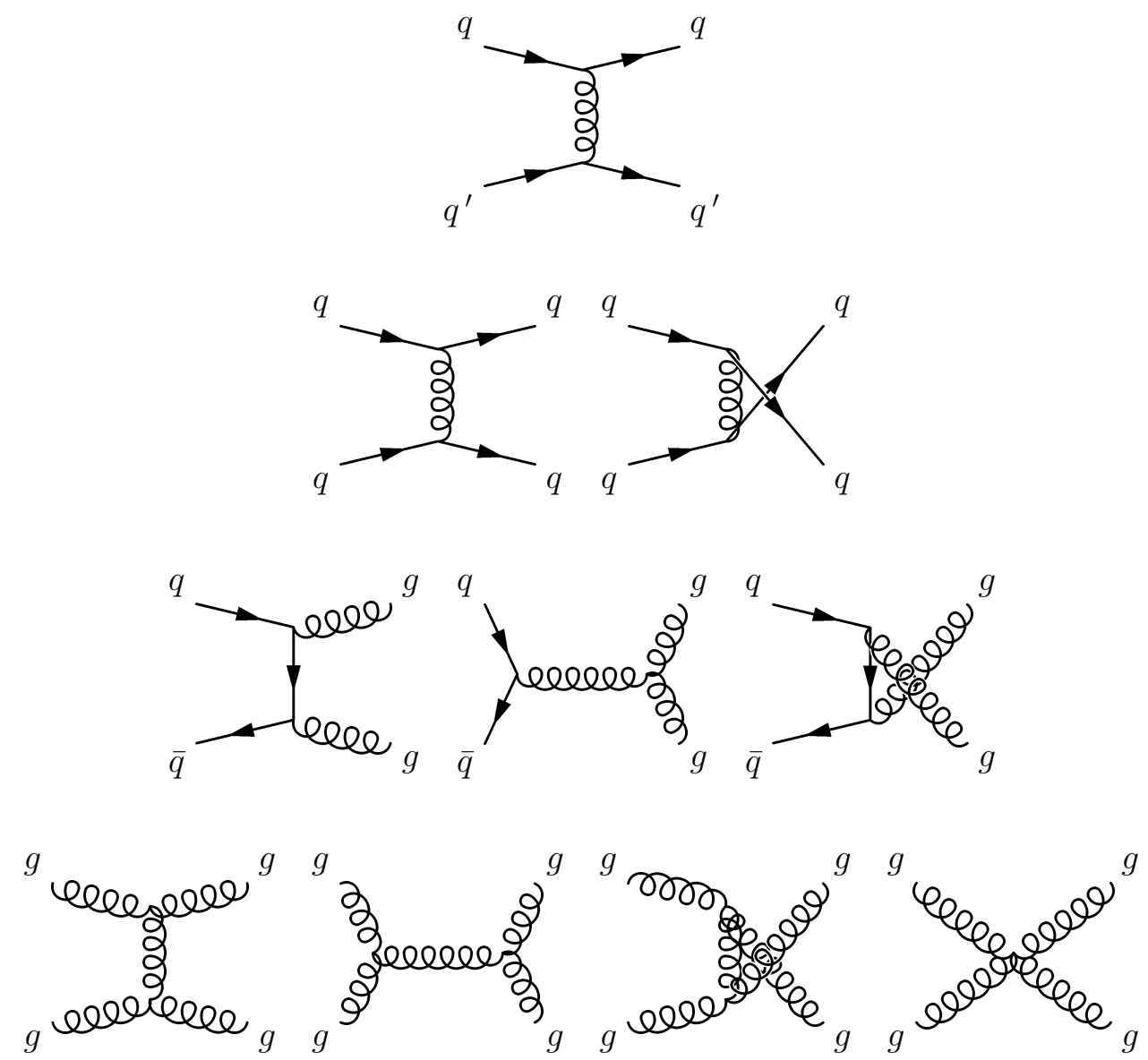

Fig. 1.3: Leading-order diagrams for resolved photoproduction of jets, corresponding to the parton scattering processes $q q^{\prime} \rightarrow q q^{\prime}, q q \rightarrow q q, q \bar{q} \rightarrow g g$ and $g g \rightarrow g g$.

\section{Prompt Photon Production}

In the regime of photoproduction the production of photons in hard interactions is referred to as prompt photon production. Both direct and resolved photoproduction processes contribute. The process of prompt photon production is sensitive to the partonic structure of the proton and due to the resolved component also to the parton content of the photon. Example diagrams of prompt photoproduction at leading order are displayed in figure 1.4.

\section{Radiative Processes}

Apart from the production in hard interactions of photoproduction processes, hard photons may arise from the next-to-leading order QED corrections on the electron-proton scattering process involving the radiation of real photons.

Figure 1.5 shows the four diagrams contributing to the neutral current scattering, corresponding to the initial and final state radiation from both the electron and quark lines. 


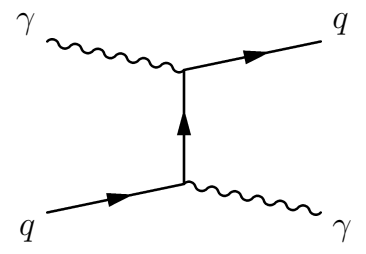

(a)

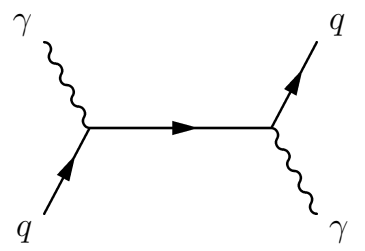

(b)

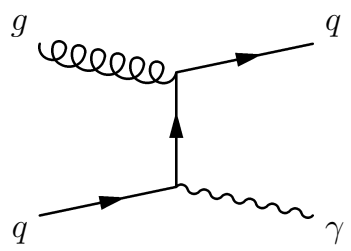

(c)

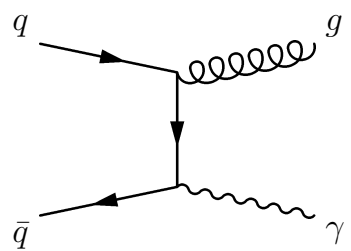

(d)

Fig. 1.4: Example diagrams for direct (a-b) and resolved (c-d) prompt photon production processes at leading order.

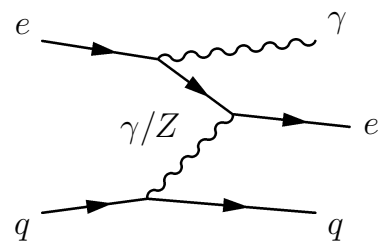

(a)

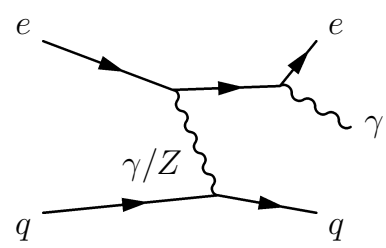

(b)

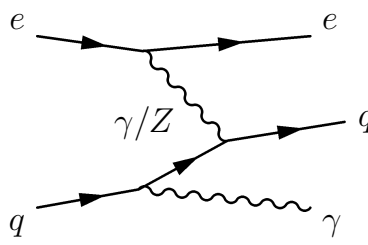

(c)

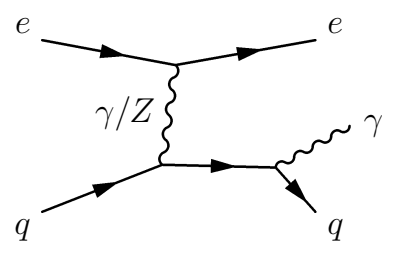

(d)

Fig. 1.5: The diagrams for real photon radiation in neutral current $e p$ scattering. Figure (a) and (b) represent the initial and final state photon radiation from the electron line, diagrams (c) and (d) the corresponding processes for the quark line.

The diagrams for charged current interactions look similar, except for the fact that photon radiation from the electron line can only proceed in the initial state. Since the cross section for the emission of real photons depends inversely proportional at least on the logarithm of the mass of the radiating particle, the radiation processes from the quark lines are suppressed due to the relative large effective quark masses.

The radiation of real photons proceeds in both elastic and inelastic scattering processes. Depending on the kinematics of the interaction the radiated photon receives large transverse momentum and is thus directly observable in the measurement.

Figure 1.6 shows the diagrams for the initial and final state radiation processes in electromagnetic ep scattering, now including the assignment of the four-momenta to the participating particles. With the following definitions of the four-momenta transfered by the intermediate photon and the virtual electron states

$$
\begin{aligned}
\hat{Q}^{2} & =-\hat{q}^{2}=-\left(p^{\prime}-p\right)^{2} \\
Q^{\prime 2} & =-q^{\prime 2}=-(k-g)^{2} \\
Q^{\prime \prime 2} & =-q^{\prime \prime 2}=\left(k^{\prime}+g\right)^{2}
\end{aligned}
$$

the double differential cross sections for the initial and final state radiation processes in electromagnetic ep scattering are determined by

$$
\frac{d^{2} \sigma}{d \hat{Q}^{2} d Q^{\prime 2}} \propto \frac{1}{\hat{Q}^{2}\left(Q^{\prime 2}+m_{e}^{2}\right)} \quad \text { and } \quad \frac{d^{2} \sigma}{d \hat{Q}^{2} d Q^{\prime \prime 2}} \propto \frac{1}{\hat{Q}^{2}\left(Q^{\prime \prime 2}+m_{e}^{2}\right)} \quad \text {. }
$$



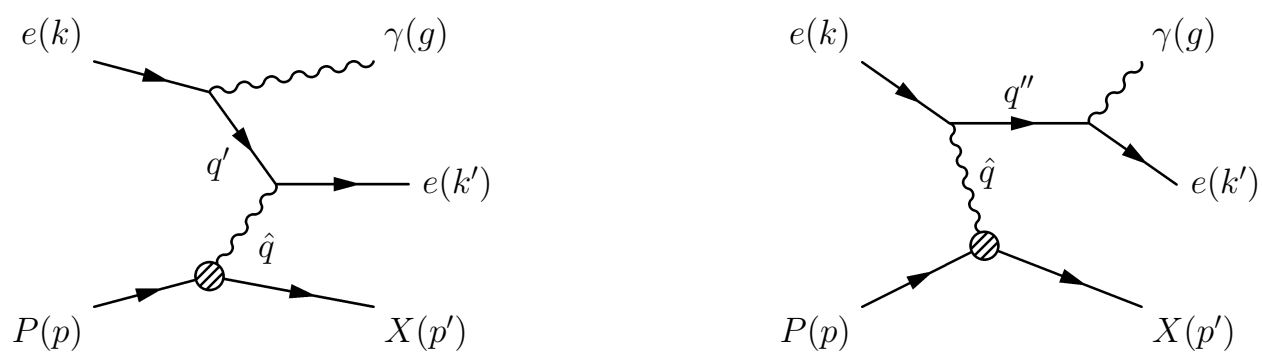

Fig. 1.6: The diagrams for the initial and final state photon radiation in electromagnetic ep scattering.

The cross sections are getting large close to the poles of the virtual photon and virtual electron states. Moreover, the size of the transfered momenta determines the relative transverse momenta of the scattered electron and radiated photon and thus the event signature in the measurement.

Based on the relative values of the transfered momenta $Q^{\prime 2}, Q^{\prime \prime 2}$ and $\hat{Q}^{2}$ the following processes are distinguished:

Small Angle Bremsstrahlung $\left({Q^{\prime 2}}^{2}, Q^{\prime \prime 2} \rightarrow 0 ; \hat{Q}^{2} \rightarrow 0\right)$ As a result of the small transfered momenta both the scattered electron and radiated photon arise at very small angles. In the elastic case $e p \rightarrow e \gamma p$ the process is known as Bethe-Heitler process and is accurately computable within the QED. Since further the event rate is large, BetheHeitler events are used at the H1 experiment to experimentally measure the luminosity (cf. section 2.2.5). The according inelastic bremsstrahlung processes represent radiative photoproduction processes.

QED Compton Scattering $\left(Q^{\prime 2}, Q^{\prime 2} \gg 0 ; \hat{Q}^{2} \rightarrow 0\right)$ Bremsstrahlung processes, where the transfered momentum $\hat{Q}^{2}$ is small $\left(\lesssim 4 \mathrm{GeV}^{2}\right)$ but the scattered electron and radiated photon emerge at large angles, are referred to as QED Compton scattering. Consequently these processes are also known as wide angle bremsstrahlung. The QED Compton scattering is dominated by elastic electron-proton interactions where the mediated photon features quasi-real character. These processes exhibit a typical event signature, since they include two electromagnetic particles which are balanced in their transverse momenta.

Radiative Deep-Inelastic Scattering $\left(Q^{\prime 2}, Q^{\prime \prime 2} \gtrsim 0 ; \hat{Q}^{2} \gg 0\right) \quad$ In this kinematic domain the diagrams shown in figure 1.6 represent the real QED corrections at the electron line to the deep-inelastic electromagnetic scattering process. Due to the characteristics of the propagator terms in (1.27) the photon emission proceeds for both the initial and final state radiation processes preferably collinear to the direction of the incoming and scattered electron, respectively $\left(Q^{\prime 2}, Q^{\prime \prime 2} \approx 0\right)$. If the photon is radiated in the initial state the electron momentum is decreased, resulting in a reduction of the effective centre-of- 


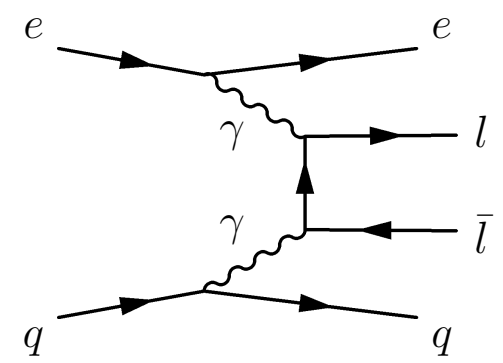

(a)

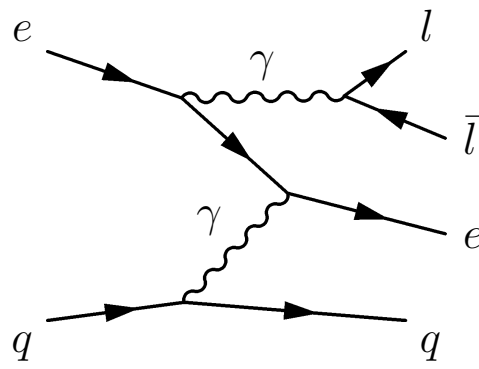

(b)

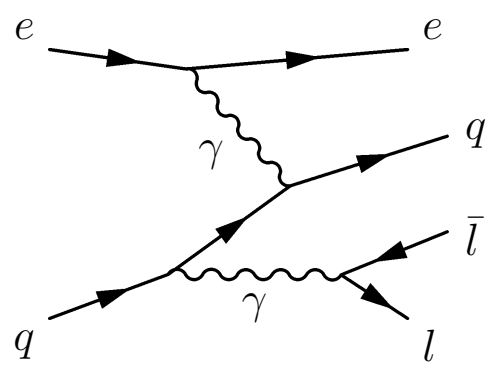

(c)

Fig. 1.7: Diagrams for lepton pair production in inelastic electromagnetic ep scattering. Figure (a) shows the t-channel diagram of the two-photon process. The radiation processes displayed in figure (b) and (c) are known as Cabibbo-Paresi and Drell-Yan process, respectively.

mass energy available in the subsequent scattering process. The radiated photon mostly remains undetected in the primal direction of the electron. In case of final state radiation both electromagnetic particles typically form a common energy cluster preventing the experimental discrimination between the electron and photon. For both processes the photon becomes observable in the measurement with increasing values of $Q^{\prime 2}$ and $Q^{\prime \prime 2}$.

\subsubsection{Lepton Pair Production}

Lepton pairs are produced in electroweak electron-proton interactions in both elastic and inelastic scattering processes. The dominant production mechanism is the two-photon reaction as depicted in figure 1.7 (a). Due to the characteristics of the photon propagator the momentum transfered to the scattered particles is generally small.

The cross section of this process can be calculated in good approximation from the convolution of the photon fluxes at the electron and proton side with the cross section of the central two-photon subprocess. While the photon flux from the electron has already been presented in section 1.1.3 the description of the flux of photons radiated from the proton is more complicated since the partonic structure of the proton has to be taken into account. Its explicit calculation can for instance be found in [14].

Another less efficient source of lepton pairs are radiation processes involving the conversion of the radiated photon or $Z^{0}$ boson into lepton pairs. Figure 1.5 has already introduced the four possible diagrams of real photon radiation in neutral current interactions, corresponding to the initial and final state radiation from the electron and quark lines. In the general electroweak case all photon propagators in the diagrams can be replaced by the propagator term of a $Z^{0}$ boson.

For both the radiation processes from the electron and quark lines the initial state radiation producing lepton pairs dominates the corresponding final state processes. In case of the boson being radiated from the initial electron, the process corresponds to an $e^{+} e^{-}$ 
annihilation into lepton pairs, in which one of the incident electrons is emitted from a photon radiated from the proton. This mechanism is known as Cabibbo-Paresi process and displayed in figure 1.7 (b). The size of the corresponding cross section and therewith its contribution to the total lepton pair production cross section depends on the flavour of the produced leptons.

When the boson is radiated from the initial quark line the underlying lepton pair production process can be regarded as the annihilation of a quark pair into a lepton pair. This process is referred to as Drell-Yan process and the corresponding diagram is shown in figure 1.7 (c). Its total contribution is small compared with the two-photon and CabibboParesi processes, nevertheless it gains in importance at high transverse momenta of the leptons.

\subsubsection{W Production}

Real $W$ bosons are produced in the inelastic electron-proton scattering either via the process $e p \rightarrow e W X$ or the process $e p \rightarrow \nu W X$. The $W$ production is dominated by the $e W$ process, which has a cross section roughly an order of magnitude larger than the process $e p \rightarrow \nu W X$ [15]. Subsequent to its production the $W$ boson decays into a fermion pair, either a charged lepton and its corresponding neutrino or a quark pair.

At the parton level the process $e p \rightarrow e W X$ is described by the diagrams presented in figures 1.8 (a-c) and figures 1.8 (f-g) and the crossing related diagrams with an antiquark in the initial state. The processes displayed in diagrams (a) and (b) can be viewed as neutral current interactions with an additional radiation of a $W$ boson from the quark in the initial or final state, respectively. Due to the presence of both the photon $t$-channel and the fermion $u$-channel in diagram (a) this particular production mechanism involving the initial state $W$ radiation dominates already the total cross section.

Diagram (c) includes a $W W \gamma$ triple boson vertex. Diagrams (f) and (g) feature a $W$ boson radiated from the lepton line, both processes are strongly suppressed by the presence of a second $W$ propagator term. Finally, diagrams (d) and (e) are needed in order to preserve electromagnetic gauge invariance when considering the subsequent decay of the $W$ boson into a fermion pair. 


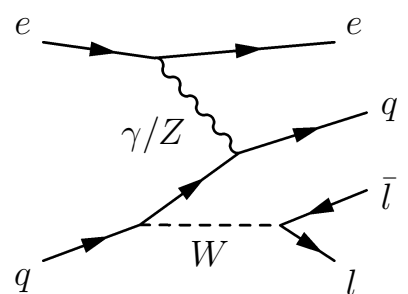

(a)

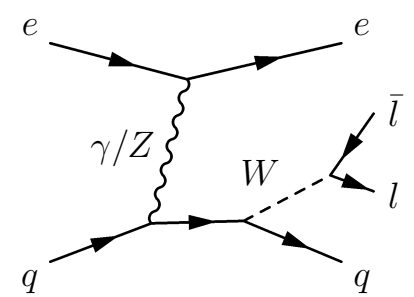

(b)

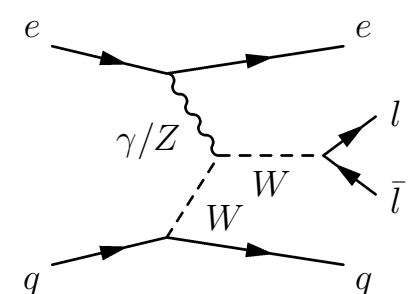

(c)

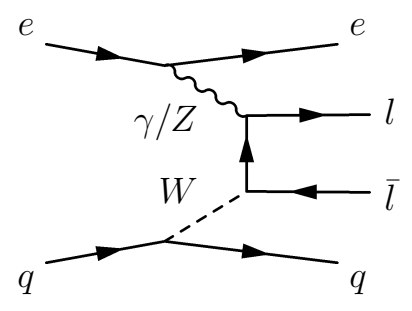

(d)

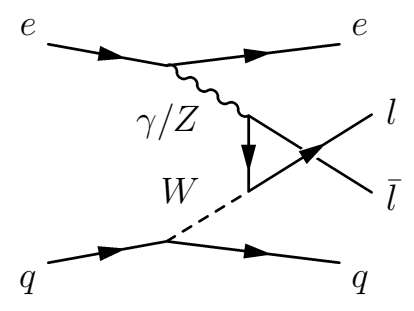

(e)

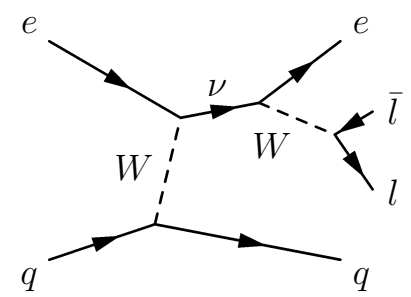

(f)

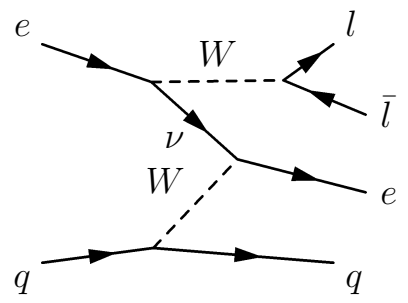

$(\mathrm{g})$

Fig. 1.8: Diagrams for real $W$ production via the process $e p \rightarrow e W X$, including the subsequent decay into fermion pairs. 


\section{Chapter 2}

\section{The H1-Experiment at HERA}

The Deutsches Elektronen-Synchrotron DESY was founded in 1959 at Hamburg in Germany as an autonomous foundation of common law with the objective of basic research in natural science. While initially established as a particle physics facility for German universities it has developed into a research centre of international renown.

The emphases of the present work at DESY are the investigation of fundamental properties of matter and forces within the scope of high energy physics as well as the research with synchrotron radiation within various fields of activity, such as solid state physics, material science, chemistry, molecular biology and medical science.

High energy physics experiments at DESY were taken up in 1964 at the electron synchrotron, which has given its name to the research centre until today. Up to the beginning of the eighties the research program continued at the $e^{+} e^{-}$storage rings DORIS $^{1}$ and PETRA $^{2}$. One of the most important discoveries at DESY, the direct evidence of gluons, succeeded at PETRA by the observation of three-jet events in 1979. Both facilities are operated for the research with synchrotron radiation today.

Apart from its operational mode as synchrotron radiation source the PETRA collider is used as pre-accelerator for the storage ring HERA, which has been commissioned in 1991 . Until this day the research in high energy physics at DESY is taking place at HERA, the first and only accelerator colliding electrons on high-energetic protons worldwide.

\subsection{The Electron-Proton Accelerator HERA}

At HERA electrons with an energy of $27.6 \mathrm{GeV}$ collide with protons at an energy of $920 \mathrm{GeV}$. The resulting centre-of-mass energy of $319 \mathrm{GeV}$ exceeds those reached in previous experiments colliding leptons on fixed proton targets by more than a factor of ten and provides therewith a much deeper insight into the proton.

As a result of the different particle masses the electron and proton beams of HERA are stored in two separate magnetic rings, which are placed in a tunnel of $6336 \mathrm{~m}$ circumfer-

\footnotetext{
${ }^{1}$ Doppel-Ring-Speicher

2 Positron-Elektron-Tandem-Ring-Anlage
} 

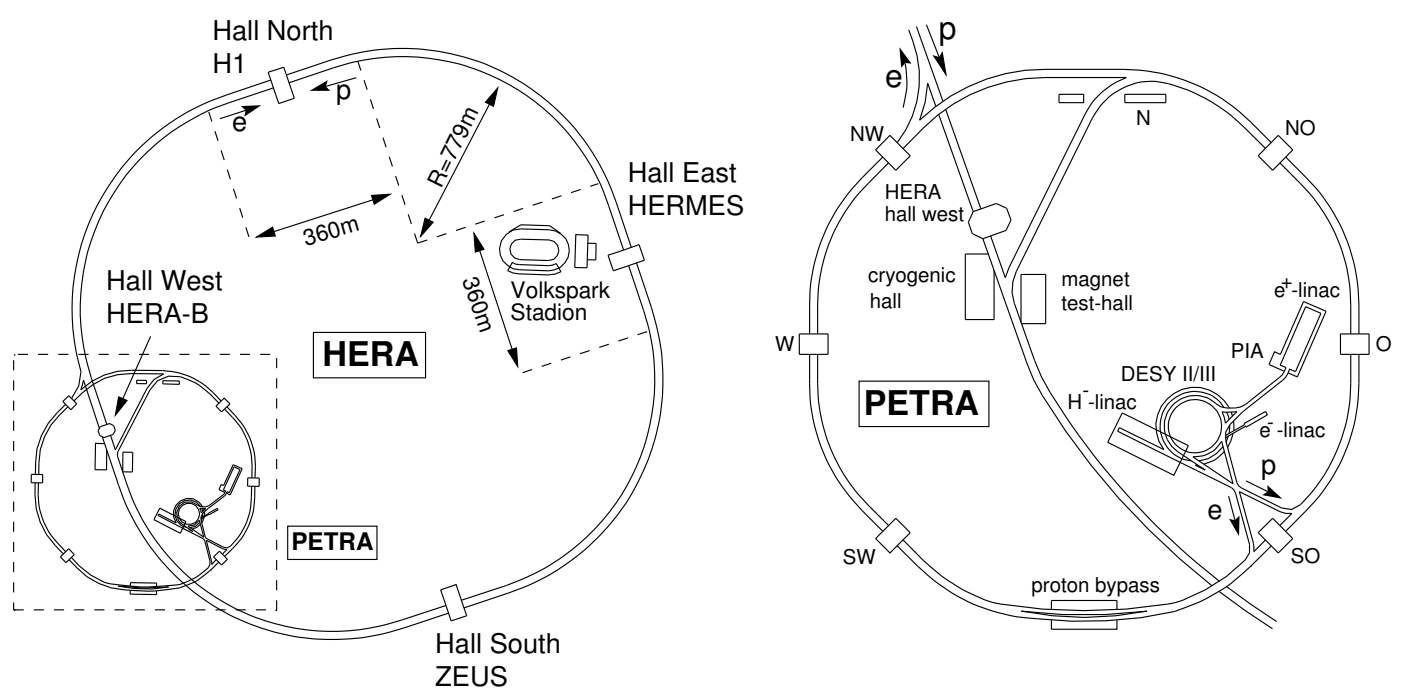

Fig. 2.1: The electron proton storage ring HERA (left) and the pre-accelerators at the DESY terrain (right). Electrons: linear accelerator LINAC II $(450 \mathrm{MeV})$, synchrotron DESY II $(8 \mathrm{GeV})$, synchrotron PETRA II $(12 \mathrm{GeV})$. Protons: linear accelerator LINAC II $(50 \mathrm{MeV})$, synchrotron DESY III $(7.5 \mathrm{GeV})$, synchrotron PETRA II $(40 \mathrm{GeV})$.

ence between 10 and $25 \mathrm{~m}$ below grade. Before being injected into HERA the electron and proton beams are passed through several pre-accelerators situated at the DESY terrain. Experimental halls are located at four places of the HERA tunnel including the detectors of the experiments H1 (Hall North), ZEUS (Hall South), HERMES (Hall East) and HERA-B (Hall West). An overview of the HERA storage ring and its pre-accelerators is presented in figure 2.1 .

While the colliding experiments $\mathrm{H} 1$ and ZEUS investigate the high-energetic scattering process of electrons and protons, either the electron or the proton beam of HERA are used by the fixed target experiments HERMES and HERA-B. The HERMES experiment started operations in 1995 and studies phenomena related to the spin of nucleons in collisions of the longitudinal polarised electron beam with polarised gas targets. The HERA-B experiment finalised data taking in 2003. It studied collisions of high-energetic protons with the nuclei of target wires positioned in the halo of the HERA proton beam. The experiment was optimised to measure a violation of the combined charge conjugation and parity conservation (CP) in decays of $B$ mesons.

The achievable energy of the electron beam is restricted by the loss resulting from synchrotron radiation. Since the amount of radiated energy is scaling with the fraction of particle energy and mass to the power of four, no significant increase of the electron energy above $30 \mathrm{GeV}$ can be reached at HERA.

The proton energy is mainly restricted by the reachable strength of the magnetic fields guiding the beam, since the energy loss due to radiation can be neglected for heavy parti- 


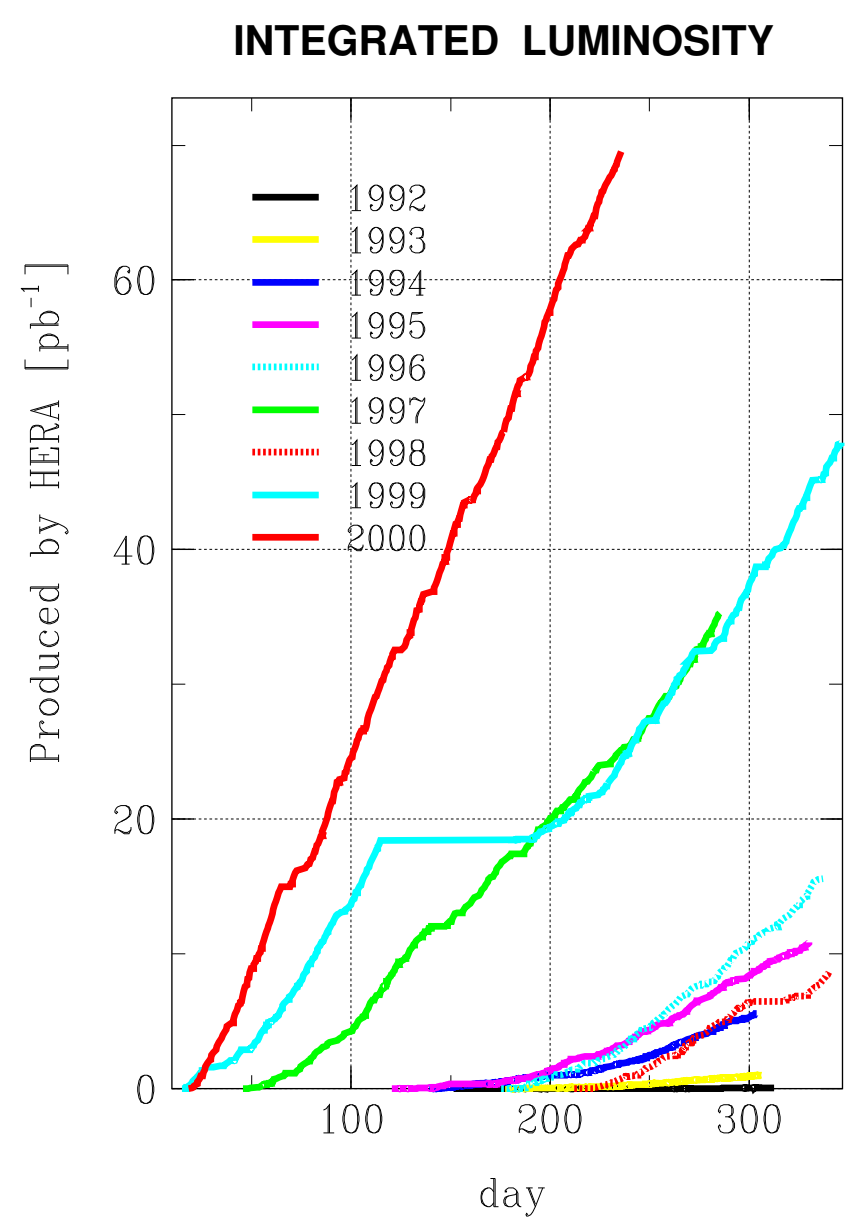

Fig. 2.2: The integrated luminosity delivered by HERA as a function of the running time during the first data taking period between 1992 and 2000 (HERA-I).

cles $^{3}$. The proton storage ring at HERA is solely equipped with superconducting magnets in order to store high-energetic protons. The main dipole magnets deliver a nominal field of $4.7 \mathrm{~T}$, about three times as strong as those attainable using normal iron yokes and copper coils.

As a result of the acceleration by cavity resonators the electron and proton beams consist of single bunches, each containing $10^{10}-10^{11}$ particles. About 180 bunches are stored at the same time, resulting in typical currents of $40 \mathrm{~mA}$ for electrons and $90 \mathrm{~mA}$ for protons. The design bunch spacing at HERA amounts to $96 \mathrm{~ns}$ corresponding to a bunch crossing rate of $10.4 \mathrm{MHz}$.

Since the HERA collider started operations in 1992 the delivered luminosity has steadily increased (cf. figure 2.2). With a specific luminosity of $\mathcal{L}=0.2 \times 10^{32} \mathrm{~cm}^{-2} \mathrm{~s}^{-1}$ HERA has exceeded its design luminosity in its first data taking period (HERA-I), which ended in autumn 2000. In order to increase the luminosity by roughly a factor of four and to give

\footnotetext{
${ }^{3} \Delta E_{e} / \Delta E_{p} \approx 10^{13} / 1$ assuming equal beam energies.
} 
the colliding experiments H1 and ZEUS access to rare physics processes, an upgrade of the interaction regions has been implemented providing a stronger focusing of the beams. HERA has successfully resumed operations end of 2003.

The presented analysis is based on the HERA-I data taken between 1994 and 2000 with the H1 experiment.

\subsection{The H1 Detector}

The basic research objectives pursued at the $\mathrm{H} 1$ experiment are the measurement of the internal structure of the proton, the study of fundamental forces acting between particles as well as the search for new physics beyond the Standard Model. In order to distinguish between the wide variety of physics processes and to obtain accurate measurement results, the identification of all reaction products must be ensured.

The universal layout of the $\mathrm{H} 1$ detector allows for the precise measurement of electrons and photons as well as muons and hadronic jets. The particles are classified according to the degree of interaction they have with the detector material. The analysis of electron-proton reactions producing weakly interacting particles, such as neutrinos arising from charged current processes, requires a hermetic detector layout in order to infer the existence of those particles indirectly from the conservation of energy and momentum.

As a result of the different beam energies the centre-of-gravity of the electron-proton system is boosted in direction of the proton beam, leading to an asymmetric detector layout. Figure 2.3 presents an isometric view of the $\mathrm{H} 1$ detector. The asymmetric layout is reflected by the enforced amount of material in the forward direction.

The origin of the H1 coordinate system is the nominal electron-proton interaction point, with the direction of the proton beam defining the positive $z$-axis (forward region). The $x$-axis and the $y$-axis point towards the centre of the HERA ring and in upward direction, respectively.

The following section gives an overview of the layout of the H1 detector. Subsequently the main detector components are described in more detail.

\subsubsection{General Layout}

The universal detector of the H1 experiment [16] is designed in order to record the maximum number of electron-proton reactions and to detect as many of the reaction products as possible. The interaction point where the particle beams collide is thus almost entirely surrounded by layers of highly sensitive detecting equipment.

The innermost layer of the H1 detector is build up by a system of tracking devices, which detects the interaction point as well as the decay positions of short lived particles with high precision and records the tracks left behind by charged particles. A magnetic field deflects the flight direction of those charged particles and allows their momentum to be determined. The inner tracking system, subdivided into a forward (fig. 2.3, (3)), central 


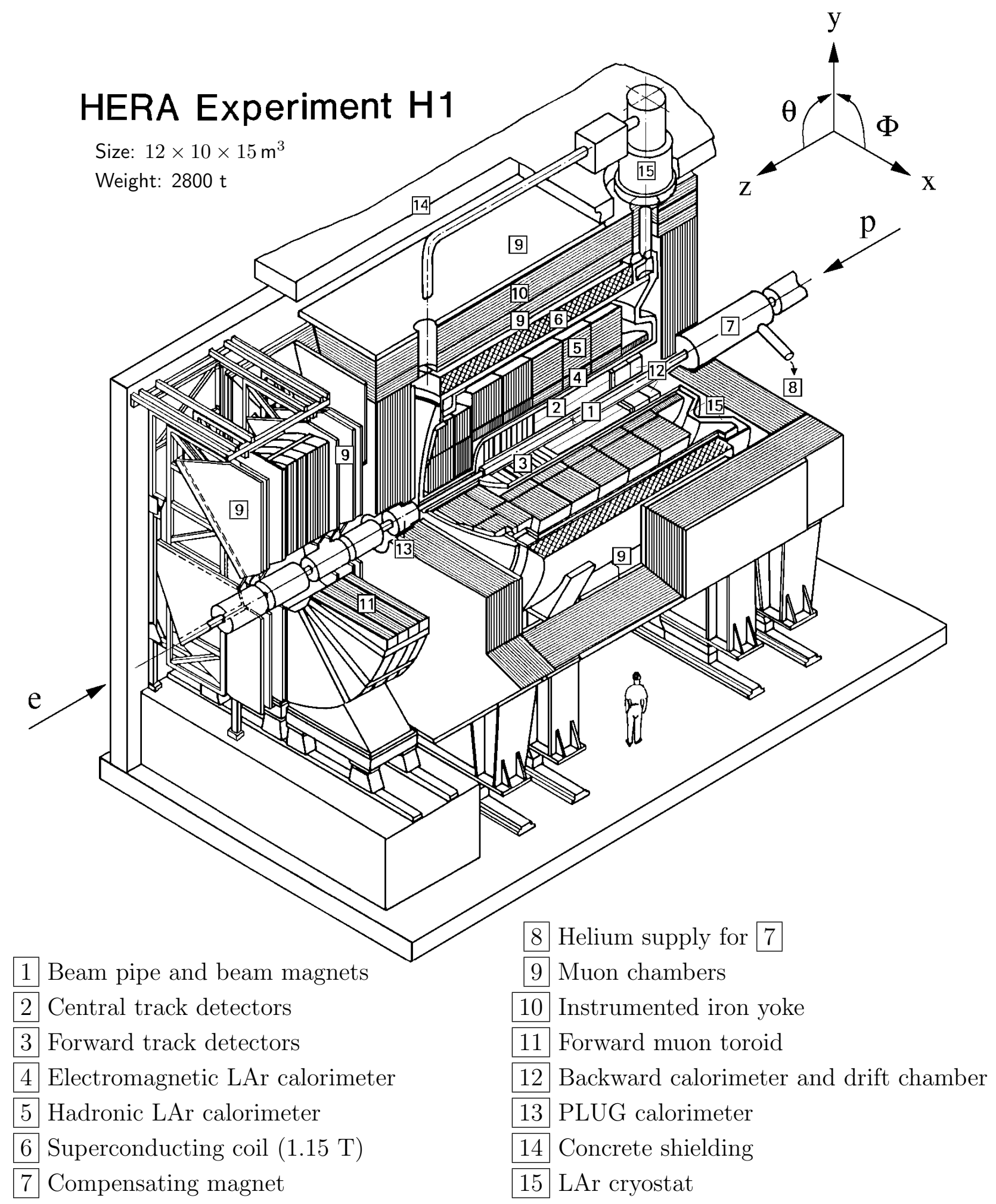

Fig. 2.3: Isometric view of the H1 detector at HERA. 
(fig. 2.3, (2)) and backward part (fig. 2.3, (12)), consists of drift chambers and multiwire-proportional chambers. In addition silicon detectors are used since 1997 in order to increase the resolution of the track measurement.

The inner tracking system is enclosed by the main calorimeter of the H1 detector, the liquid argon (LAr) calorimeter, which provides the energy measurement of the final state particles in the central region. Typically, the innermost electromagnetic section (fig. 2.3, (4)) measures the particle showers produced by electrons and photons in the detector material, while the outer section (fig. 2.3, (5)) detects the remaining hadrons.

In order to measure hadrons scattered under small angles the PLUG calorimeter (fig. 2.3, (13)) is assembled in forward direction and completes the hadronic calorimetry of the H1 detector close to the beam pipe. Electrons scattered under small angles and hadronic activity in backward direction are detected by the $\mathrm{BEMC}^{4}$ (fig. 2.3, (12)) up to the end of 1994. This electromagnetic lead-scintillator sampling calorimeter has been replaced by the $\mathrm{SpaCal}^{5}$, a lead-scintillating fibre calorimeter providing a larger polar acceptance as well as higher energy resolution.

The calorimetry of the $\mathrm{H} 1$ detector is completed by the so-called Tail Catcher calorimeter, whose components are implemented in the Instrumented Iron Yoke (fig. 2.3, (10)) and measure hadronic showers leaking out of the LAr calorimeter.

The magnetic field needed for the momentum measurement of tracks is provided by a large superconducting solenoid (fig. 2.3, (6)), which encloses the LAr calorimeter and inner tracking system. The solenoid is assembled in a helium cryostat and provides a magnetic field of $1.15 \mathrm{~T}$ strength aligned parallel to the beam direction. The iron yoke is used to channel the fields in the coils and their interference with the beam is prevented by a compensator magnet (fig. 2.3, (7)) assembled close to the beam pipe in backward direction.

The outermost layer of the $\mathrm{H} 1$ detector is equipped with components detecting muons, which can penetrate dense layers of material without being absorbed. The muon system is subdivided into the Forward Muon Spectrometer, consisting of a toroidal magnet (fig. 2.3, (11)) surrounded by drift chambers (fig. 2.3, (9)), and the Central Muon Detector, whose limited streamer tubes are embedded in the Instrumented Iron Yoke (fig. 2.3, (10)) enclosing all major components of the $\mathrm{H} 1$ detector. The muon detection is completed by muon chambers (fig. 2.3, (9)) mounted on both sides of the iron yoke.

\subsubsection{Inner Tracking System}

The demands on the inner tracking detectors are the precise measurement of momentum and flight direction of charged particles as well as the accurate determination of the interaction point and secondary vertices. Since the final state particles of the interaction are typically collimated in forward direction, the inner tracking system is subdivided into three major components optimised for the measurement in different polar angle re-

\footnotetext{
${ }^{4}$ Backward Electromagnetic Calorimeter

${ }^{5}$ Spaghetti Calorimeter
} 


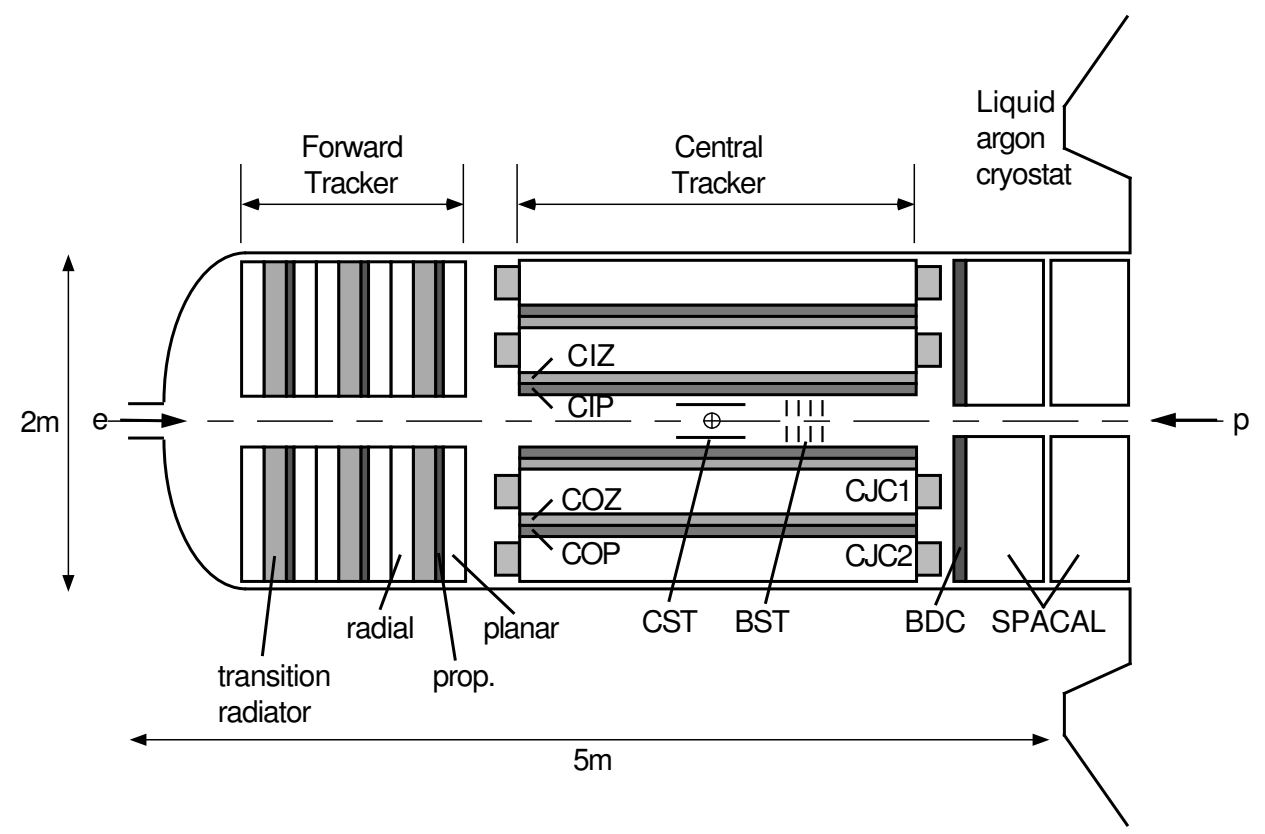

Fig. 2.4: Side view of the inner tracking system ( $r z$-plane).

gions. The inner tracking system covers nearly the complete range in polar angle with full azimuthal acceptance. A general view of the system is shown in figure 2.4.

\section{Forward Tracking System}

Tracks in the forward direction are measured by the Forward Tracker, which covers the polar angle range of $7^{\circ}<\theta<25^{\circ}$. The detector consists of three so-called super-modules, each composed of planar drift chambers, a multi-wire-proportional chamber, transition radiation detectors as well as a radial drift chamber.

\section{Backward Tracking System}

The proportional chamber BWPC has been used to measure tracks in backward direction up to the end of 1994. Along with the backward calorimeter (cf. section 2.2.3) the BWPC has been replaced the Backward Drift Chamber BDC, which extended the polar acceptance to $153^{\circ}<\theta<177.5^{\circ}$.

\section{Central Tracking System}

The central tracking system is subdivided into an inner and outer section, each consisting of a large drift chamber, a proportional chamber as well as a so-called $z$-chamber. Since 1997 two micro-vertex detectors are used in order to improve the resolution of track reconstruction and accuracy of the vertex determination. 


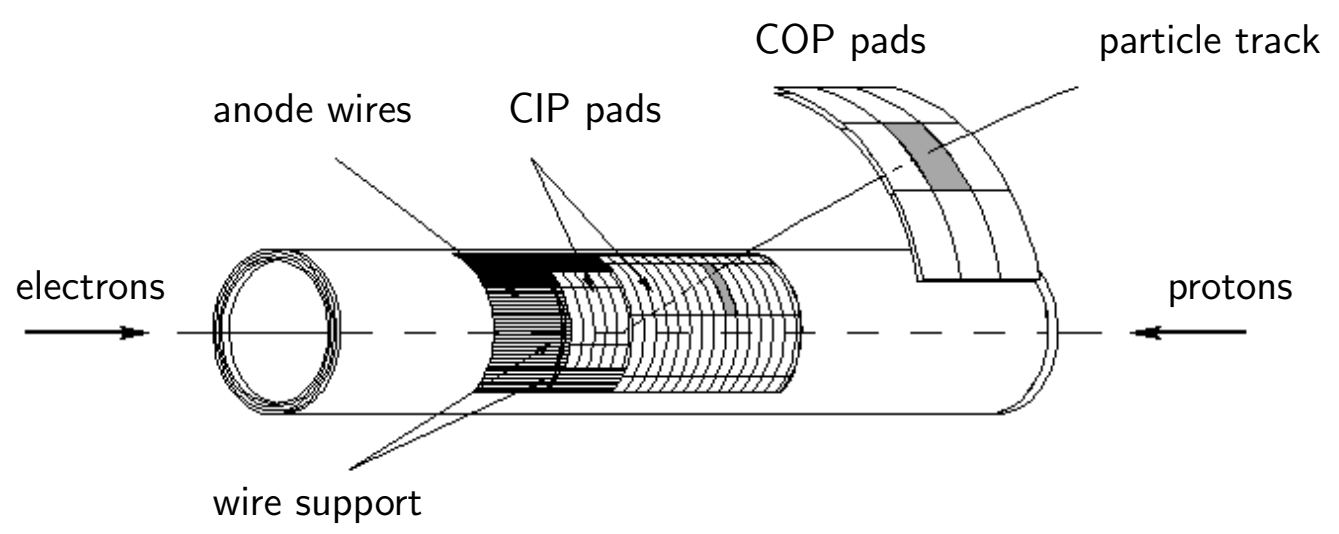

Fig. 2.5: Isometric view of the CIP detector.

Central Jet Chambers The track reconstruction in the central region is based on the measurement of the Central Jet Chambers CJC1 and CJC2. These two cylindrical drift chambers cover a polar angle range of $15^{\circ}<\theta<165^{\circ}$. In total 2640 sense wires aligned parallel to the beam axis ensure a high accuracy in the position determination of charged tracks in the $r \phi$-plane, resulting in a transverse momentum resolution of $\delta p_{T} / p_{T}<0.01 p_{T} / \mathrm{GeV}$. Based on the drift time measurement the interaction time $T_{0}$ of the event is determined with a precision of one nano second. Particle identification is provided by the measurement of the specific energy loss $d E / d x$.

Central $\boldsymbol{Z}$-Chambers The measurement of the $z$-coordinate of charged tracks is improved by the information delivered from the inner and outer $z$-chambers CIZ and COZ. These two drift chambers with wires aligned perpendicular to the beam axis enclose the inner jet chamber CJC1 and provide a typical resolution of $300 \mu \mathrm{m}$.

Central Proportional Chambers The central $z$-chambers are surrounded by the inner and outer proportional chambers CIP and COP. These multi-wire-proportional chambers deliver fast trigger signals and support the reconstruction of the $z$-coordinate of the interaction vertex. In addition the information obtained from the CIP is used in this analysis to improve the electron and photon identification (cf. section 4.3.1 and 4.3.2) by either the requirement or applying a veto on hits left behind. The CIP covers the polar angle range of $5^{\circ}<\theta<175^{\circ}$ and consist of two active chamber layers centred around the beam axis in the radius of $15.7 \mathrm{~cm}$ and $16.2 \mathrm{~cm}$, respectively. The read out cathodes of each layer are separated into 480 pads with a length of $3.6 \mathrm{~cm}$ in $z$-direction and a azimuthal coverage of $45^{\circ}$. Both layers are rotated with respect to each other by an angle of $11.25^{\circ}$ in azimuthal direction, each layer contains 480 anode wires aligned parallel to the beam axis. 
Micro-Vertex Detectors The central tracking system is completed by two micro-vertex detectors, which are mounted closest to the interaction point inside the Central Tracker, fully commissioned in 1997. The Central Silicon Tracker CST consist of two cylindrical layers equipped with silicon strip detectors, concentrically surrounding the beam axis in the central region. The CST is supported by a second micro-vertex detector situated in backward direction. The Backward Silicon Tracker BST consists of eight layers of silicon strip detectors oriented perpendicular to the beam direction. The high spatial resolution of silicon detectors significantly improves the measurement of the interaction vertex as well as the resolution of the track reconstruction and allows for the determination of secondary vertices.

\subsubsection{Calorimetry}

The calorimetry section of the $\mathrm{H} 1$ detector provides the energy measurement of the final state by as complete as possible absorption of the particles within the detector material. In order to cover the full solid angle the calorimeter system is divided into various subdetectors.

The main component is represented by the LAr calorimeter providing the energy measurement in the forward and central polar angle range of $4^{\circ}<\theta<154^{\circ}$. The backward region was covered by the BEMC up to the end of 1994, which has then been replaced by the SpaCal with an polar acceptance of $153^{\circ}<\theta<177.5^{\circ}$. Hadronic activity in the very forward direction is measured by the PLUG calorimeter closing the acceptance gap between the LAr calorimeter and the beam pipe.

The calorimetry system of the H1 detector is completed by the components of the Tail Catcher calorimeter (cf. section 2.2.4), embedded in the iron yoke, and the detectors of the luminosity system (cf. section 2.2.5), located downstream of the interaction point far away in the HERA tunnel. The latter calorimeters provide apart from their main task, i.e. the determination of the luminosity, the tagging of photoproduction events as well as the energy measurement of small-angle scattered electrons and radiative photons from initial state radiation. Between 1998 and end of 2000 the $\mathrm{VLQ}^{6}$ calorimeter allowed for the measurement of electrons scattered in backward direction into the polar angle range of $178.3<\theta<179.4$.

\section{LAr Calorimeter}

As well as most other calorimeters of the $\mathrm{H} 1$ detector the LAr calorimeter is assembled in sampling structure, denoting the alternation of absorber plates and active material. Intruding particles are induced to shower while penetrating the absorber material. The outcoming secondary particles ionise the active material and produce further showers within the absorber plates. The electrons released in the ionisation are forced to readout

\footnotetext{
${ }^{6}$ Very Low $Q^{2}$
} 


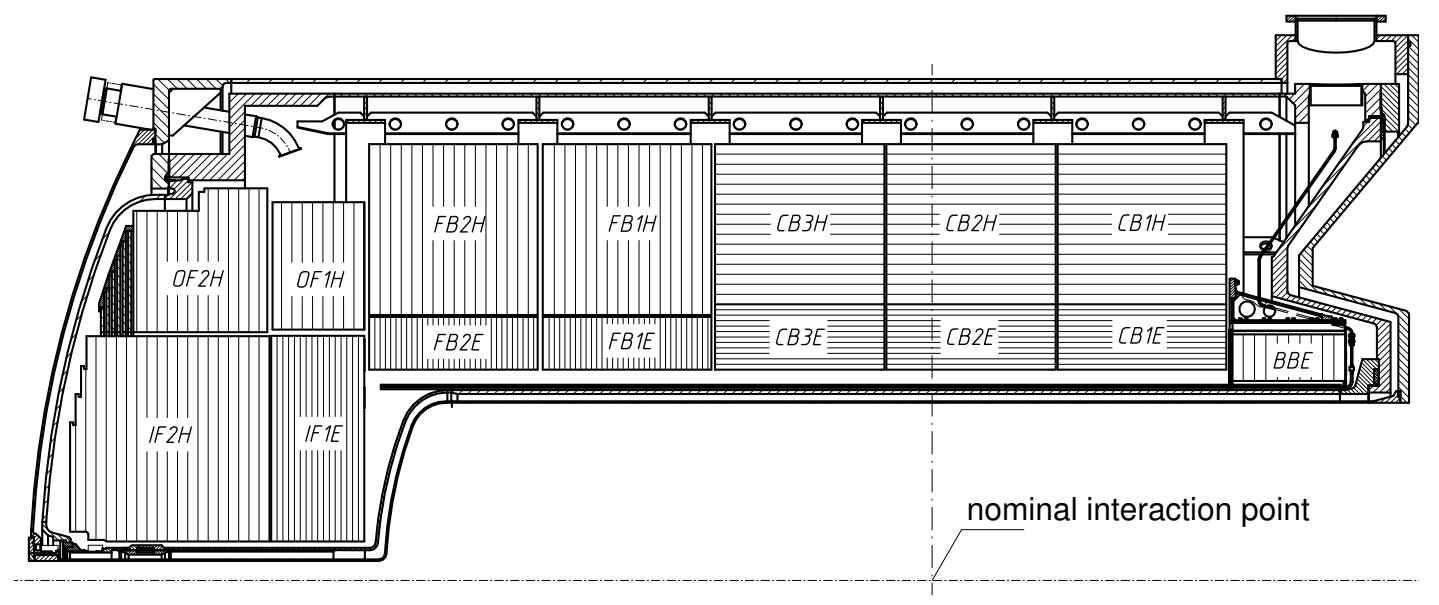

IF

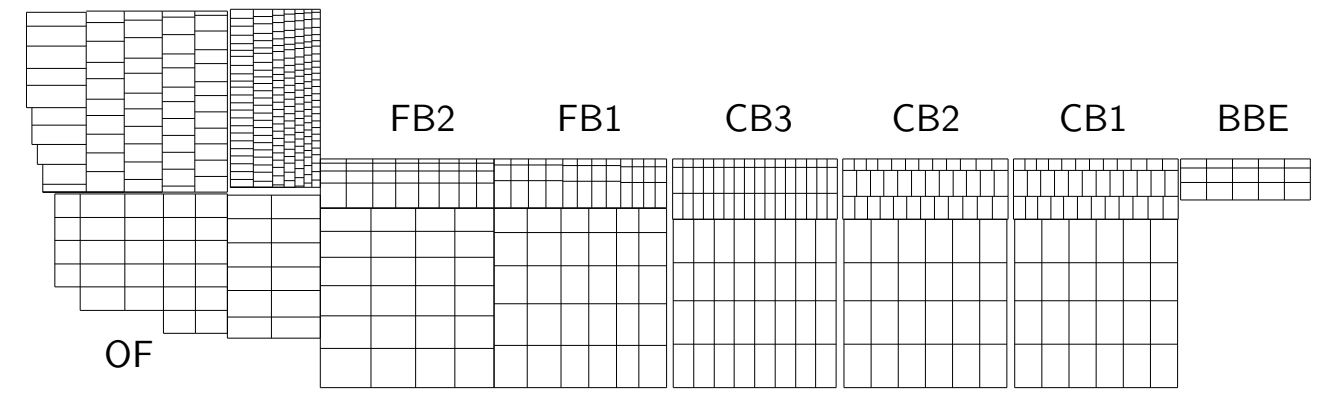

Fig. 2.6: Side view of the LAr calorimeter ( $r z$-plane). The eight wheels are labelled with BBE, CB1, CB2, CB3, FB1, FB2, OF and IF. The modules of the inlying electromagnetic section and the outer hadronic section are distinguished by the labels $(\mathrm{E})$ and $(\mathrm{H})$, respectively. The orientation of the absorber plates is displayed in the upper part of the figure while the lower part shows the cell configuration used in the read out.

planes by a strong electric field, producing signals proportional to the energy of the intruding particles.

The LAr calorimeter is composed of an inner electromagnetic section enclosed by an outer hadronic part. Liquid argon at $90^{\circ} \mathrm{K}$ is used as active material in both sections, ensuring stable performance, ease of calibration as well as homogeneity in the calorimeter response. In the electromagnetic section the absorbing material is made of lead, while steel absorber plates are used in the hadronic section. The absorber plates are mounted such that the enclosing angle of penetrating particles exceeds $45^{\circ}$ in each region of the calorimeter. The orientation of the absorber plates is displayed in the upper part of figure 2.6.

The LAr calorimeter consists of eight self-supporting wheels, each composed of eight modules (cf. figure 2.6). These calorimeter wheels are contained in a single cryostat. In order to minimise the amount of dead material in front of the calorimeter and to improve the energy resolution, the LAr calorimeter is mounted inside the superconducting coil providing the magnetic field for the track measurement.

Both sections of the LAr calorimeter are non-compensating, where compensation denotes the balance of the signal response to electrons and hadrons with the same energy. There- 
fore an off-line weighting technique is applied on the measured energies to account for the non-compensating nature of the LAr calorimeter.

\section{Backward Calorimeter SpaCal}

Since 1995 the SpaCal provides the energy measurement in the backward region of the $\mathrm{H} 1$ detector. Compared to the former installed BEMC it covers a larger polar angle range and offers a higher energy resolution.

Likewise the LAr calorimeter the SpaCal consists of an inner electromagnetic and outer hadronic section, each with an active depth of $25 \mathrm{~cm}$ centred around the beam axis (cf. figure 2.3 and 2.4). The front of the calorimeter has a diameter of $160 \mathrm{~cm}$ and is situated at a $z$-position of $-1.50 \mathrm{~m}$. Scintillating fibres oriented parallel to the beam axis are used as active material, densely enclosed by an absorber matrix made of lead.

The electromagnetic section consists of 1192 cells with a surface of $4.05 \times 4.05 \mathrm{~cm}^{2}$. Each cell is connected to a photo-multiplier, forming a single readout unit. The hadronic section contains 136 cells with a surface of $12.00 \times 12.00 \mathrm{~cm}^{2}$. The innermost part of the SpaCal is formed by a special insert composed of 16 calorimeter cells, which measures the amount of energy leaking out of the electromagnetic section.

The SpaCal is characterised by the high energy resolution of its electromagnetic section, reaching values below $2 \%$ for energies around $30 \mathrm{GeV}$.

\subsubsection{Central Muon Detector}

The Central Muon Detector CMD forms the outermost hermetic component of the H1 detector and provides the measurement of muons exceeding a minimum momentum between 1 and $2 \mathrm{GeV}$. The sensitive components of the system are embedded in the slits as well as on the inner and outer surface of the Instrumented Iron Yoke. As a results of the layout of the iron yoke the CMD is geometrically subdivided into four sectors, the two end-caps and the forward and backward barrel. Each of those sub-components consists of 16 detector modules (cf. upper part of figure 2.7).

While the measures of the modules depend on the location in the detector, their general layout is the same. The cross section of a module located in the backward barrel sector is presented in figure 2.7. Each module contains at least 9 sensitive layers situated between the plates of the iron yoke. In order to improve the track reconstruction and to cover the edges of the detector, each three additional layers contained in aluminium boxes may be mounted inside and outside the iron yoke for dedicated modules.

Two combined types of sensitive units are used for the detection of penetrating muons. Layers composed of limited streamer tubes deliver the two-dimensional spatial information, while strip electrodes installed perpendicular to the direction of the wires contained in the streamer tubes provide the third coordinate.

Additional pad segmented electrode foils installed on dedicated layers provide a rough measurement of deposited energy. Those pads form the so-called Tail Catcher calorimeter 


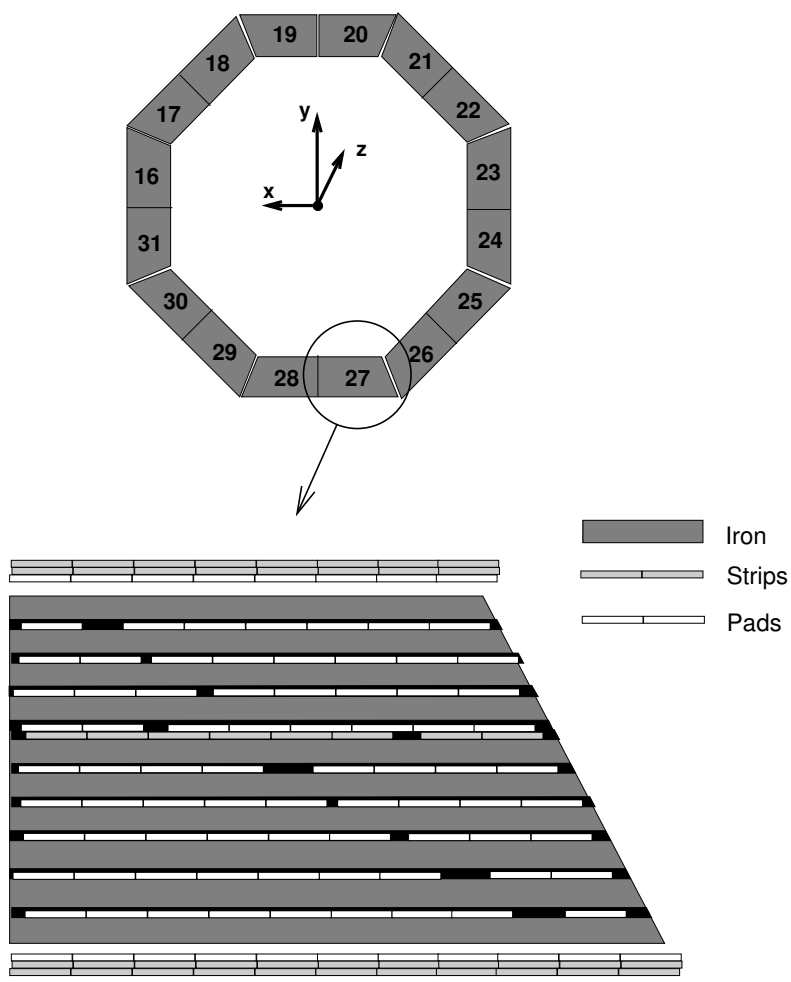

Fig. 2.7: Cross section of a module located in the backward barrel sector of the Central Muon Detector.

(cf. section 2.2.3) and measure the energy leaking out of the LAr calorimeter.

\subsubsection{Luminosity System}

The integrated luminosity is determined for a given physical process from the relation between the cross section and the number of observed events by

$$
\mathcal{L}_{\text {int }}=\frac{N_{\text {obs }}}{\sigma_{\text {process }}}
$$

In order to experimentally measure the luminosity the so-called Bethe-Heitler process $e p \rightarrow e p \gamma$ is used at HERA (cf. section 1.2.2). Since the cross section of this small-angle bremsstrahlung process is large and theoretically precisely computable, the luminosity can be determined from the accurate measurement of the according event rate.

Contrary to the central components of the $\mathrm{H} 1$ detector described above the sub-detectors used for the luminosity measurement are situated up to $100 \mathrm{~m}$ away from the interaction point in the HERA tunnel. The luminosity system consists of the electron tagger (ET) at a $z$-position of $-33.4 \mathrm{~m}$ and the photon detector (PD) at $-102.8 \mathrm{~m}$, which measure the scattered electron and collinear radiated photon from Bethe-Heitler events in coincidence. Both detectors use Čerenkov-crystal-calorimeters, which are read out by photomultipliers. The side view of the luminosity system is shown in figure 2.8 . 


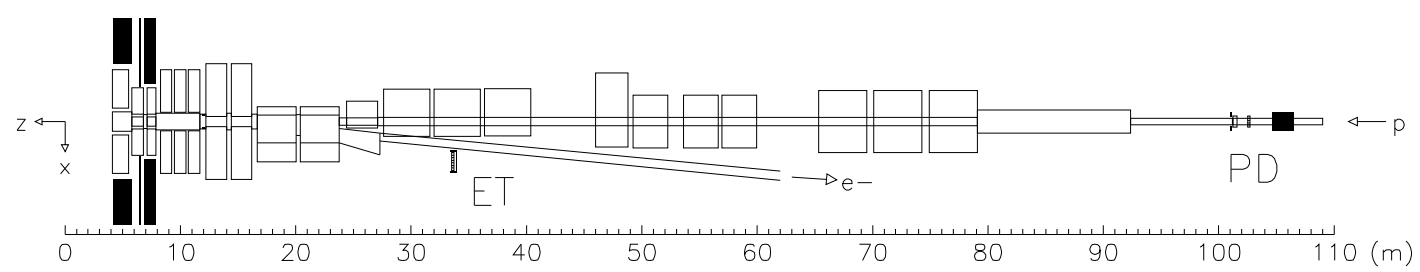

Fig. 2.8: Side view of the luminosity system of the H1 detector.

\subsection{Trigger and Data Acquisition}

The spectra of physics processes accessible in the observation of electron-proton collisions at HERA covers multiple orders of magnitude in cross section. The resulting event rates range from $20-30 \mathrm{~Hz}$ in the region of photoproduction down to approximately $10^{-5} \mathrm{~Hz}$ for the production of $W$ bosons $^{7}$. Anyhow, the highest rates in the order of $50 \mathrm{kHz}$ arise from background reactions such as synchrotron radiation of the electron beam and interactions of the protons with either the wall of the beam pipe (beam-wall events) or with remaining gas atoms inside the beam pipe (beam-gas events). In order to heavily reduce the amount of recorded background events and to adjust the rate at which certain event signatures are selected, a fast trigger system is needed choosing those events to be stored permanently on magnetic tapes for further analyses.

Most of the H1 sub-detectors provide binary coded signals to be used on trigger level. These so-called trigger elements are combined to physics subtriggers in oder to specify the event signature and to extract those events of particular physics interest from the large amount of background.

The trigger system of the $\mathrm{H} 1$ detector consists of four levels, which reduce the incoming rate of $10.4 \mathrm{MHz}$ determined by the HERA bunch spacing stepwise down to $5-10 \mathrm{~Hz}$.

Trigger Level L1 The first trigger level L1 processes the incoming data without dead time. Since the trigger decision on L1 takes about $2 \mu \mathrm{s}$ in time, the read out of the subdetectors is organised using a pipeline structure, typically storing the information on the last 32 events. In case of being accepted by at least one subtrigger the continuous read out of the detector front end electronics is stopped, initiating the dead time of the experiment since no new event is recorded.

Trigger Level L2 If having passed the first level the event is further analysed by the second trigger level L2 within $20 \mu \mathrm{s}$. The L2 system is composed of the two independent components L2TT and $\mathrm{L}_{2} \mathrm{NN}^{8}$, applying a more sophisticated analysis based on the information of different sub-detectors available at this stage. If the event is accepted by

\footnotetext{
7 The given values are based on the HERA design luminosity of $\mathcal{L}=1.5 \cdot 10^{31} \mathrm{~cm}^{-2} s^{-1}$.

${ }^{8}$ Level 2 Topological Trigger and Neural Network
} 
L2 the full read out of all sub-detectors is initiated. At the end of the read out after approximately $1 \mathrm{~ms}$ the $\mathrm{H} 1$ data taking is resumed and the dead time completed.

Trigger Level L3 The third trigger level L3 allows for further investigations of the events within a time of $2 \mathrm{~ms}$, but it was not used during the HERA-I data taking period.

Trigger Level L4 The reduced incoming rate of $30-40 \mathrm{~Hz}$ is handled by the forth trigger level L4, providing a nearly full event reconstruction carried out by a processor farm. In contrast to the first and second trigger level the data processing on L4 proceeds no more in time with the HERA clock. Selection cuts are applied on the reconstructed quantities, assigning the accepted events into certain physical classes.

The full reconstruction of the events selected by the trigger system is carried out by the $\mathrm{H} 1$ reconstruction software package H1REC. Based on the raw data information delivered by the $\mathrm{H} 1$ sub-detectors physical quantities such as particle tracks or calibrated energies are calculated. The final processed data are stored on magnetic tapes in the form of POT's ${ }^{9}$, including the full event information, and in a more compressed format as so-called DST's ${ }^{10}$ and are then available to the physics working groups for further analyses.

\subsection{Reconstruction of the Kinematics}

The kinematic quantities $Q^{2}, x, y$ and $W^{2}$ used in the theoretical description of the scattering process of electrons on protons (cf. section 1.1.1) allow for a precise and relativistic invariant formulation of the subjects matter but are not directly accessible to a measurement. At a given centre-of-mass energy $\sqrt{s}$ the kinematics of the deep-inelastic electron-proton scattering is determined by two independent variables.

The $\mathrm{H} 1$ detector provides the measurement of both the energy and angle of the scattered electron as well as the energy and angle of the particles included in the hadronic final state. Utilising this redundancy of the measurement various possibilities exist in order to reconstruct the event kinematics from these four independently determined observables. At least two of them are combined by a certain reconstruction method to determine two independent Lorentz invariant variables. As a result of the varying precision at which the input quantities are determined in the measurement the resolutions of the kinematic quantities differ for various reconstruction methods.

In the following the Electron Method, the Hadron Method and the Double Angle Method are exemplarily presented.

\footnotetext{
${ }^{9}$ Production Output Tape

${ }^{10}$ Data Summary Tape
} 


\section{The Electron Method}

The Electron Method [17] is based on the measurement of the energy $E_{e}$ and polar angle $\theta_{e}$ of the scattered electron and is thus only qualified for the reconstruction of neutral current events. The kinematic variables are given by

$$
\begin{aligned}
Q_{e}^{2} & =2 E_{0} E_{e}\left(1+\cos \theta_{e}\right), \\
y_{e} & =1-\frac{E_{e}}{2 E_{0}}\left(1-\cos \theta_{e}\right), \\
x_{e} & =\frac{Q_{e}^{2}}{y_{e} s}
\end{aligned}
$$

where $E_{0}$ denotes the electron beam energy. While the Electron Method provides a precise reconstruction of $Q^{2}$ over the full kinematic range, the resolutions of $x$ and $y$ significantly decrease at low values of $y$.

\section{The Hadron Method}

The Hadron Method [18] also known as Jacquet-Blondel Method relies only on the measurement of the hadronic final state in order to reconstruct the full event kinematics:

$$
\begin{aligned}
Q_{\text {had }}^{2} & =\frac{\left(\sum_{h} p_{x, h}\right)^{2}+\left(\sum_{h} p_{y, h}\right)^{2}}{1-y_{\text {had }}}, \\
y_{\text {had }} & =\frac{\sum_{h}\left(E_{h}-p_{z, h}\right)}{2 E_{0}}, \\
x_{\text {had }} & =\frac{Q_{\text {had }}^{2}}{y_{\text {had }} s}
\end{aligned}
$$

where the sum runs over all particles detected in the final state and $E$ and $p_{x}, p_{y}, p_{z}$ denote their energy and momentum components, respectively. Even though the resolutions of the kinematic quantities are typically poor compared to the Electron Method, the Hadron Method allows for the reconstruction of processes where the scattered lepton escapes undetected from the measurement, as it is the case for charged current events or in the regime of photoproduction.

\section{The Double Angle Method}

The Double Angle Method [17] uses only the polar angle of the scattered electron and the angle $\gamma$ which characterises the hadronic final state:

$$
\begin{aligned}
Q_{d a}^{2} & =4 E_{0}^{2} \frac{\sin \gamma\left(1+\cos \theta_{e}\right)}{\sin \gamma+\sin \theta_{e}-\sin \left(\theta_{e}+\gamma\right)} \\
y_{d a} & =\frac{\sin \theta_{e}(1-\cos \gamma)}{\sin \gamma+\sin \theta_{e}-\sin \left(\theta_{e}+\gamma\right)} \\
x_{d a} & =\frac{Q_{d a}^{2}}{y_{d a} s}
\end{aligned}
$$


The hadronic angle $\gamma$ is defined by

$$
\cos \gamma=\frac{\left(\sum_{h} p_{x, h}\right)^{2}+\left(\sum_{h} p_{y, h}\right)^{2}-\left(\sum_{h}\left(E_{h}-p_{z, h}\right)\right)^{2}}{\left(\sum_{h} p_{x, h}\right)^{2}+\left(\sum_{h} p_{y, h}\right)^{2}+\left(\sum_{h}\left(E_{h}-p_{z, h}\right)\right)^{2}}
$$

where the sum runs over all particles of the hadronic final state. This reconstruction method has the advantage, that it does not rely on the precise knowledge of the absolute calorimetric energy calibration. However, variations in the energy scale with the polar angle will distort the measurement of the hadronic angle $\gamma$. 


\section{Chapter 3}

\section{Monte Carlo Simulation}

The basic intention of analyses accomplished in the field of high energy physics is to improve the understanding of the fundamental interactions between elementary particles by comparing theoretical predictions, manifested in the Standard Model, with the results from measurements. Since any experimental measuring device is limited in acceptance and resolution, its influence on the physical observables must be considered in order to draw universal conclusions from the results of a specific experiment.

Within the so-called Monte Carlo technique extensive software packages are used to simulate dedicated physical processes and the according detector response. Based on theoretically calculated cross sections Monte Carlo event generators deliver the four-momenta of the final state particles. Processes not computable within the framework of perturbative theory, such as the fragmentation of quarks and gluons into hadrons, are approached using phenomenological models. Subsequent to the generation of events the detector response is determined by the simulation software.

\subsection{Monte Carlo Generation}

A general search for deviations from the Standard Model at HERA, as presented in this analysis, requires a precise and reliable estimate of all relevant electron-proton scattering processes. Since no single Monte Carlo event generator containing all processes is available, several programs are used to generate events for all dominant SM contributions, carefully avoiding double-counting of processes.

All processes are generated in the dominantly contributing regions of phase space with a luminosity significantly higher than that of the analysed data sample. In order to ensure a precise description up to the borders of phase space, the generated luminosity is significantly increased in those kinematic regions.

The following paragraphs describe the considered processes and their Monte Carlo generation. This analysis investigates the phase space region of high transverse momenta. In this kinematic regime the dominating SM processes are the photoproduction of two jets and the neutral current deep-inelastic scattering. 
In the following the abbreviation $X$ denotes the system of the reaction products not belonging to a high transverse momentum final state composed of electrons $(e)$, photons $(\gamma)$, muons $(\mu)$, jets $(j)$ or neutrinos $(\nu)$. Processes with additional jets (e.g. ep $\rightarrow j j j X$ or $e p \rightarrow e j j j X)$ are accounted for in all generators using leading logarithmic parton showers or the colour dipole approach as representation of higher order QCD radiation. The prediction of neutral current, charged current and photoproduction processes with two or more high transverse momentum jets (e.g. $e p \rightarrow j j X$ or $e p \rightarrow e j j X$ ) is scaled by a factor of 1.2 to re-weight the normalisation of the leading order Monte Carlos to that of next-to-leading order QCD calculations [19].

\section{Photoproduction of Jets and Photons}

The PYTHIA event generator [20] is used to simulate the direct and resolved photoproduction of jets $e p \rightarrow j j X$, the production of prompt photons $e p \rightarrow \gamma j X$ and the tiny cross section of resolved photoproduction of photon pairs $e p \rightarrow \gamma \gamma X$. Light (uds) and heavy flavours (cb) are generated. The simulation contains the Born level hard scattering matrix elements and radiative QED corrections. Higher order QCD effects are accounted for by leading logarithmic parton showers. The hadronization of the outcoming partons is based on the Lund String model [21]. The parton density function (pdf) GRV LO [22] is used on the photon side and the CTEQ5L [23] pdf is chosen for the proton.

\section{Neutral Current Deep-Inelastic Scattering}

The Born, QCD Compton and Boson Gluon Fusion matrix elements are implemented in the RAPGAP [24] event generator to model neutral current DIS events. QED radiative effects due to real photon emission from both the incoming and outcoming electron as well as one-loop virtual corrections are simulated using the HERACLES [25] generator. Hence the neutral current DIS prediction contains the processes ep $\rightarrow e j X$, ep $\rightarrow$ ejjX and $e p \rightarrow e \gamma j X$. In case of the electron having low transverse momentum RAPGAP contributes to $e p \rightarrow j j X$ and $e p \rightarrow \gamma j X$. The generation of parton cascades is based on the leading logarithmic parton shower approach, accounting for higher order QCD effects. The CTEQ5L pdf is used on the proton side to evaluate the nominal NC cross section.

\section{Charged Current Deep-Inelastic Scattering}

Charged current DIS events are calculated using the DJANGOH [26] program, which includes first order QED corrections based on HERACLES. This prediction contains the processes $e p \rightarrow \nu j X, e p \rightarrow \nu j j X$ and processes with an additional photon radiation. Parton cascades are generated using the colour dipole model in ARIADNE [27]. The CTEQ5L pdf is chosen to evaluate the nominal CC cross section. 


\section{QED Compton Scattering}

Elastic and quasi-elastic Compton processes $e p \rightarrow e \gamma X$ are simulated with the WABGEN [28] generator. The inelastic contribution is already included in the NC DIS RAPGAP sample.

\section{Electroweak Production of Lepton Pairs}

Multi-lepton events are generated with GRAPE [29], which includes all exact electroweak matrix elements at tree level. Multi-lepton production via $\gamma \gamma, \gamma Z$ and $Z Z$ collisions as well as internal photon conversion and via the decay of virtual or real $Z$ bosons is considered. Initial and final state QED radiation is simulated in leading logarithmic approximation. The complete hadronic final state is obtained via interfaces to PYTHIA and SOPHIA [30] for the inelastic and quasi-elastic regime, respectively. Consequently GRAPE predicts $e p \rightarrow \mu \mu X$ and $e p \rightarrow e e X$ and $e p \rightarrow e \mu \mu X$ and $e p \rightarrow e e e X$, if the scattered electron is detected, as well as processes with additional photon radiation.

\section{W Production}

The production of $W$ bosons $e p \rightarrow W X$ and $e p \rightarrow W j X$ is modelled using the EPVEC [15] event generator. Next-to-leading order QCD corrections [31] are taken into account by re-weighting the events as a function of the transverse momentum and rapidity of the $W$ boson [32]. The ACFGP [33] pdf is chosen on the photon side while the CTEQ4M [34] pdf is used for the proton. The simulation of final state parton showers is based on the PYTHIA software package.

\subsection{Simulation}

All generated events are passed through a full detector simulation using the H1 simulation program H1SIM based on the GEANT [35] software package. Taking into account the geometrical setup and material parameters of each sub-component the response of the $\mathrm{H} 1$ detector on penetrating particles is determined. All secondary particles produced are tracked stepwise in their way through the detector and the interaction with the material is simulated based on the according cross sections.

In order to reduce the amount of computing time the simulation of electromagnetic and hadronic showers in the calorimeters is performed using the H1FAST mode. The most important simplifications therein are the replacement of detailed geometrical structures by homogenous material distributions as well as the description of electromagnetic energy deposits using parameterised energy density functions [36].

Likewise the real data the simulated events are reconstructed using the H1 software package H1REC and subject to the same analysis chain in order to compare the measurement with the prediction provided by the Standard Model.

A summary of the Monte Carlo samples used in this analysis is presented in table 3.1. 


\begin{tabular}{|c|c|c|c|c|}
\hline Process(es) & Generator & Phase Space & Subprocess & $\mathcal{L}_{\mathrm{MC}} / \mathcal{L}_{\text {data }}$ \\
\hline Photoproduction & PYTHIA & $\begin{array}{l}\hat{P}_{T}>5 \mathrm{GeV} \\
\hat{P}_{T}>10 \mathrm{GeV} \\
\hat{P}_{T}>10 \mathrm{GeV} \\
\hat{P}_{T}>15 \mathrm{GeV} \\
\hat{P}_{T}>25 \mathrm{GeV} \\
\hat{P}_{T}>25 \mathrm{GeV} \\
\hat{P}_{T}>40 \mathrm{GeV} \\
\hat{P}_{T}>75 \mathrm{GeV} \\
\hat{P_{T}}>95 \mathrm{GeV}\end{array}$ & $j-j-j-j(*)$ & $\begin{array}{r}0.4 \\
1.5 \\
1.5 \\
15 \\
150 \\
600 \\
150 \\
2200 \\
75000\end{array}$ \\
\hline Prompt Photon & PYTHIA & $\begin{array}{l}\hat{P_{T}}>10 \mathrm{GeV} \\
\hat{P_{T}}>20 \mathrm{GeV} \\
\hat{P_{T}}>40 \mathrm{GeV}\end{array}$ & & $\begin{array}{r}75 \\
500 \\
20000 \\
\end{array}$ \\
\hline $\mathrm{NC}$ & RAPGAP & $\begin{array}{l}Q^{2}>4 \mathrm{GeV}^{2} \\
Q^{2}>100 \mathrm{GeV}^{2} \\
Q^{2}>400 \mathrm{GeV}^{2} \\
Q^{2}>400 \mathrm{GeV}^{2}, \hat{P}_{T}>20 \mathrm{GeV} \\
Q^{2}>400 \mathrm{GeV}^{2}, \hat{P}_{T}>30 \mathrm{GeV} \\
Q^{2}>400 \mathrm{GeV}^{2}, \hat{P}_{T}>35 \mathrm{GeV} \\
Q^{2}>400 \mathrm{GeV}^{2}, \hat{P}_{T}>35 \mathrm{GeV} \\
Q^{2}>1000 \mathrm{GeV}^{2} \\
Q^{2}>2500 \mathrm{GeV}^{2} \\
Q^{2}>5000 \mathrm{GeV}^{2} \\
Q^{2}>10000 \mathrm{GeV}^{2} \\
Q^{2}>20000 \mathrm{GeV}^{2}\end{array}$ & $\begin{array}{c}e-j-j-j^{(*)} \\
e-j-j-j-j^{(*)}\end{array}$ & $\begin{array}{r}10 \\
7 \\
40 \\
1000 \\
1200 \\
26000 \\
122000 \\
12 \\
27 \\
100 \\
540 \\
9600\end{array}$ \\
\hline $\mathrm{CC}$ & DJANGOH & $\begin{array}{l}Q^{2}>100 \mathrm{GeV}^{2} \\
Q^{2}>10000 \mathrm{GeV}^{2}\end{array}$ & & $\begin{array}{r}245 \\
13000 \\
\end{array}$ \\
\hline $\begin{array}{l}\text { Lepton Pair } \\
\text { Production }\end{array}$ & GRAPE & $\begin{array}{l}M_{\mu \mu}>60 \mathrm{GeV} \\
M_{\mu \mu}>100 \mathrm{GeV}\end{array}$ & $\begin{array}{l}e-e \\
\mu-\mu \\
\mu-\mu \\
\mu-\mu \\
\tau-\tau\end{array}$ & $\begin{array}{r}450 \\
750 \\
6000 \\
85000 \\
1500\end{array}$ \\
\hline QED Compton & WABGEN & $\begin{aligned} M_{e \gamma} & >10 \mathrm{GeV} \\
M_{e \gamma} & >50 \mathrm{GeV} \\
M_{e \gamma} & >100 \mathrm{GeV}\end{aligned}$ & & $\begin{array}{r}10 \\
340 \\
5000 \\
\end{array}$ \\
\hline W Production & EPVEC & & & 1500 \\
\hline
\end{tabular}

Tab. 3.1: Monte Carlo samples used in this analysis. All processes are generated for proton energies of $820 \mathrm{GeV}$ and $920 \mathrm{GeV}$. For the ratio $\mathcal{L}_{\mathrm{MC}} / \mathcal{L}_{\text {data }}$ the smallest value found is given. The RAPGAP sample is simulated separately for the run periods $e^{+} p$ and $e^{-} p$. The subprocesses distinguished by the superscript $(*)$ have been extracted by additional requirements while generating. 


\section{Chapter 4}

\section{Data Selection and Classification}

The approach presented in this thesis consists of a comprehensive and generic search for deviations from the SM prediction at large transverse momenta. All high- $P_{T}$ final state configurations involving electrons, muons, jets and neutrinos are systematically investigated in a common analysis for the first time.

The motivation and strategy of the presented analysis are introduced in more detail in the first part of this chapter. Subsequently the event selection on which the analysis is based is presented.

The main part of the chapter is related to the experimental measurement of the final state with the H1 Detector. The identification criteria of the considered particles and the classification of events into exclusive event classes are described.

\subsection{Analysis Strategy}

The H1 experiment at HERA has accumulated data corresponding to more than $100 \mathrm{pb}^{-1}$ of integrated luminosity in the first running period between 1994 and 2000 (HERA-I) and provides therewith a complete and well understood data set.

One important goal at HERA is the search for new physics beyond the Standard Model. Although no deviation from the SM has been established, it is widely believed that the $\mathrm{SM}$ is incomplete and that new physics signals may appear below energies of $1 \mathrm{TeV}$.

A large variety of extensions to the SM has been constructed during the last decades predicting various phenomena appearing at high energies or large transverse momenta. HERA data have been used to test some of these models of new processes by analysing their anticipated experimental signatures and limits on their parameters have been derived [37, 38].

Unlike analyses searching for signals in dedicated channels predicted by specific theoretical models, the approach described in this work consists of a general search for deviations from the SM in all accessible final state configurations of ep interactions. The analysis covers phase space regions where the SM prediction is sufficiently precise to detect anomalies and does not rely on assumptions concerning the characteristics of a SM extension. 


\begin{tabular}{|c|c|c|c|c|c|}
\hline year & $\begin{array}{c}\text { data } \\
\text { sample }\end{array}$ & $\begin{array}{c}\text { electron } \\
\text { energy }\end{array}$ & $\begin{array}{c}\text { proton } \\
\text { energy }\end{array}$ & $\begin{array}{c}\text { centre-of-mass } \\
\text { energy }\end{array}$ & $\begin{array}{c}\text { integrated } \\
\text { luminosity }\end{array}$ \\
\hline \hline $1994-1997$ & $e^{+} p$ & $27.6 \mathrm{GeV}$ & $820 \mathrm{GeV}$ & $301 \mathrm{GeV}$ & $36.4 \mathrm{pb}^{-1}$ \\
$1998-1999$ & $e^{-} p$ & $27.6 \mathrm{GeV}$ & $920 \mathrm{GeV}$ & $319 \mathrm{GeV}$ & $13.8 \mathrm{pb}^{-1}$ \\
$1999-2000$ & $e^{+} p$ & $27.6 \mathrm{GeV}$ & $920 \mathrm{GeV}$ & $319 \mathrm{GeV}$ & $66.4 \mathrm{pb}^{-1}$ \\
\hline
\end{tabular}

Tab. 4.1: The HERA-I data samples used in the analysis. The integrated luminosities are calculated with respect to the used run selection and high voltage corrections.

An in this spirit called model-independent search might therefore be able to discover unexpected manifestations of new physics and give an answer to the important question, if new physics signals might be hidden in the HERA-I data.

The analysis is based on a proper and unambiguous definition of objects, which are defined by the type of particles identified in the final state using the criteria of this analysis and a requirement applied on their phase space. The considered objects are electron $(e)$, muon $(\mu)$, photon $(\gamma)$, jet $(j)$ and neutrino $(\nu)$ (or any number of non-interacting particles). A common phase space is required for all objects. The transverse momentum $P_{T}$ must exceed $20 \mathrm{GeV}$, and the polar angle $\theta$ of all particles except for the neutrino has to be reconstructed within the range of $10^{\circ}<\theta<140^{\circ}$.

In order to avoid bias, the object identification criteria are defined according to the knowledge of detector performances and signatures of SM processes only. No additional criteria related to specific final state configurations enters the event selection.

Based on the complete HERA-I data sample all events are classified into exclusive event classes according to the number and types of objects detected in the final state. All configurations including at least two isolated objects are considered (e.g. $e-j, j-j, e-j-\nu$ ). Exclusive event classes ensure a clear separation of final states and later on an unambiguous statistical interpretation of deviations.

In a first step of the analysis the global yields of the event classes are compared to the SM expectation and the distributions of the invariant mass $M_{\text {all }}$ and the scalar sum of transverse momenta $\sum P_{T}$ of the identified high- $P_{T}$ final state objects are discussed (cf. section 6). Both kinematical quantities are chosen since they are sensitive to new physics signals as well as easy to measure:

$$
M_{\text {all }}=\sqrt{\left(\sum_{i} p_{i}\right)^{2}} \quad \text { and } \quad \sum P_{T} \equiv \sum_{i} P_{T, i}=\sum_{i} \sqrt{P_{x, i}^{2}+P_{y, i}^{2}} \quad,
$$

where the sum runs over all high- $P_{T}$ objects belonging to an event class and $p_{i}, P_{T, i}$ and $P_{x, i}, P_{y, i}$ denote the four-momentum, the transverse momentum and the $x$ and $y$ momentum components of each object. Assuming that new physics will appear at the highest energies a first sign may be visible as an excess or a deficit in one of these distributions. Once a deviation has been detected its origin can be explored in dedicated analyses. In a second step these distributions are systematically investigated (cf. chapter 7). A new statistical algorithm is presented which locates the region of largest deviation from the 


\begin{tabular}{|c|}
\hline 1994-2000 data, $\mathcal{L}=116.6 \mathrm{pb}^{-1}$ \\
good, medium runs \\
major systems operational: \\
LAr, CJC1-2, Luminosity System, SpaCal, CIP and COP \\
\hline$-36<z_{v t x}<34 \mathrm{~cm}(1994-1997)$ \\
$-35<z_{v t x}<35 \mathrm{~cm}(1998-2000)$ \\
\hline$\sum_{i}\left(E_{i}-P_{z, i}\right)<75 \mathrm{GeV}$ \\
\hline timing CJC: $-11.4 \mathrm{~ns}<T_{0}<11.4 \mathrm{~ns}$ \\
timing LAr: $-67.2 \mathrm{~ns}<T_{0}<67.2 \mathrm{~ns}$ \\
\hline QBGFMAR bits 0-8 false for all classes (except $\mu$ event classes) \\
QBGFMAR bits 0-9 false for $\nu$ event classes (except $\mu$ event classes) \\
all 26 QBGFMAR bits false for $j-\nu$ and $\nu-\gamma$ event classes \\
\hline
\end{tabular}

Tab. 4.2: Basic event selection criteria.

SM prediction in the distributions. To quantitatively determine the level of agreement between the data and the SM prediction the probability of occurrence of such a deviation is derived.

\subsection{Event Selection}

At HERA positrons collide with an energy of $27.6 \mathrm{GeV}$ with protons at an energy of $920 \mathrm{GeV}$ resulting in a centre-of-mass energy $\sqrt{s}$ of $319 \mathrm{GeV}$. Before 1997 the proton energy amounted to $820 \mathrm{GeV}$ resulting in a reduced centre-of-mass energy of $301 \mathrm{GeV}$. In addition the positron beam, used in the years 1994 to 1997 and since midyear 1999 again, has been changed to electrons between 1998 and the first half of 1999 .

Combining these three data sets the examined event sample contains the full 1994-2000 HERA-I data, corresponding to an integrated luminosity of $116.6 \mathrm{pb}^{-1}$. An overview of the used sub-samples is presented in table 4.1.

In order to ensure stable detector conditions only runs with good and medium quality are extracted out of the amount of recorded data. In addition all major systems which are essential for the analysis are required to be operational. These sub-detectors are the luminosity system, the LAr and SpaCal calorimeters, the Central Jet Chambers CJC1 and CJC2 as well as the Central Proportional Chambers CIP and COP.

The basic data selection requires at least one isolated electromagnetic cluster, jet or muon to be found anywhere in the detector acceptance. Energy deposits in the calorimeters and tracks measured in the central tracking system are combined to reconstruct the hadronic energy of the events.

To reduce non-ep background events it is demanded that the event vertex is reconstructed within a distance of $35 \mathrm{~cm}$ around the nominal $z$ position of the vertex ${ }^{1}$, and the longi-

\footnotetext{
${ }^{1}$ This is not required for the event classes containing only photons (and neutrino).
} 

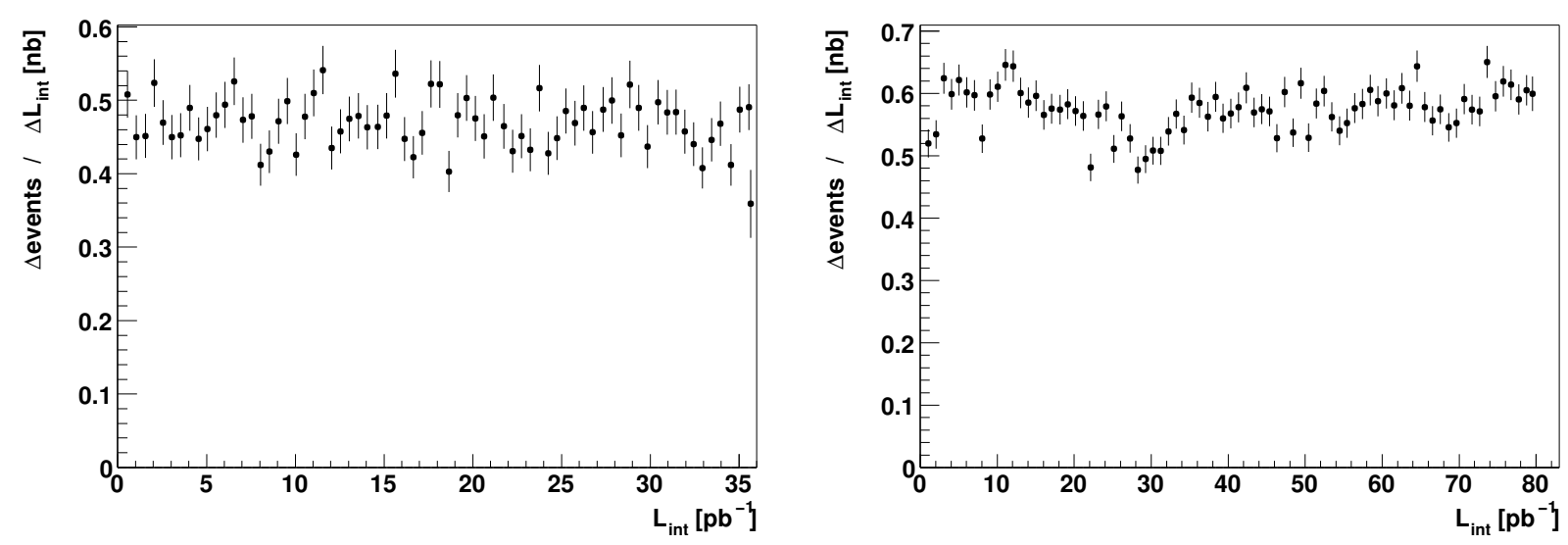

Fig. 4.1: The event yields as a function of the integrated luminosity for the run period 1994-1997 (left side) and 1998-2000 (right side) [41].

tudinal momentum balance $\sum_{i}\left(E_{i}-P_{z, i}\right)$ is restricted to values below $75 \mathrm{GeV}$, where $E_{i}$ denotes the particle energy and $P_{z, i}$ the $z$ component of the momentum. The sum runs over all hadronic energy deposits, electromagnetic clusters and muons found in the event. Due to energy-momentum conservation a HERA event is expected to have a value of $\sum_{i}\left(E_{i}-P_{z, i}\right)$ equal to $55.2 \mathrm{GeV}$, i.e. twice the electron beam energy, if the complete final state has been detected or if only longitudinal momentum along the proton direction has been undetected.

Further background is rejected by searching for event topologies typical for cosmic ray and beam-induced background events. Therefore the software package QBGFMAR [39] is used, providing 26 background finders. Since the efficiencies of these finders vary for different event topologies the requirement of the background finder bits is adjusted depending on the final state configuration [40].

Moreover the timing of the event is required to coincide with the ep bunch crossing. Both, the information from the Central Jet Chambers and the LAr calorimeter are considered.

A summary of the basic event selection criteria is presented in table 4.2. The event yield, i.e. the ratio of selected events and integrated luminosity, is shown in figure 4.1 as a function of the integrated luminosity, separately for the data samples with different proton momenta recorded in 1994-1997 and 1998-2000, respectively.

\subsection{Particle Identification}

This analysis investigates all final state configurations of $e p$ interactions with at least two objects with transverse momenta $P_{T}>20 \mathrm{GeV}$ in the polar angle range $10^{\circ}<\theta<140^{\circ}$. Considered objects are electrons, muons, photons, jets and neutrinos (or any number of non-interacting particles). In order to ensure a clear separation of the final state configurations all objects have to be isolated towards each other by a minimum distance of 
$R=\sqrt{(\Delta \eta)^{2}+(\Delta \phi)^{2}}>1$ in the pseudo-rapidity-azimuth $(\eta-\phi)$ plane $^{2}$.

Furthermore all events with an isolated high- $P_{T}$ object which is not clearly identified as electron, photon or jet are rejected to minimise false classification of events. E.g. events with electron candidates, which no track can be assigned to but also fail the photon identification criteria, are rejected.

The identification criteria for each type of particle are based on previous analyses performed on specific final states [10, 37, 38, 42]. Additional requirements were chosen to ensure an unambiguous identification of particles, still keeping high efficiencies.

The following paragraphs describe the identification criteria.

\subsubsection{Electrons}

The electron identification is based on the measurement of a compact and isolated electromagnetic shower in the LAr calorimeter associated with a track in the inner tracking system. The transverse momentum of the electron is reconstructed using the energy and polar angle information provided by the LAr calorimeter.

Electron candidates in the LAr calorimeter are identified in the present analysis using the search algorithm QECFWD [43], which is part of the electron finder QESCAT [44]. In order to discriminate electrons (and photons, cf. section 4.3.2) from hadronic particles, several conditions are applied on topological estimator variables. These conditions are related to the shape and the size of the electromagnetic shower profile of the electron candidate.

All estimators and the appropriate cut values used for the preselection of the electron candidates are summarised in table 4.3. The parameters EAEM, EAHN and EATR ensure the compactness of the selected object while the level of isolation is tested by the estimators EAIF and EAHD. If more than one electron exists in the event the candidates are chosen in decreasing order of the reconstructed transverse momentum $P_{T}$.

After the preselection of candidates by QECFWD harsher cuts are applied on the calorimetric isolation to ensure an unambiguous identification. Electron candidates reconstructed in inefficient regions of the LAr calorimeter, such as gaps between wheels ( $z$-cracks) or modules ( $\phi$-cracks), are excluded from the analysis.

A track linked to the electron cluster is required to reduce the misidentification of photons and hadrons. Thus certain $\phi$-regions are excluded from the electron identification depending on the run periods in 1997 and 1999 affected by hardware problems of the Central Jet Chambers.

Since the acceptance of the central track detector is limited in the forward region $\left(\theta<37^{\circ}\right)$ the applied cluster and track criteria depend on the reconstructed polar angle of the electron candidate.

In the very forward region $\left(10^{\circ}<\theta<20^{\circ}\right)$ only the sub-detectors CJC1 and FTD contribute to the track measurement. Since the radiation length of the dead material is large in this polar angle region the probability for electrons to shower gets high. The short

\footnotetext{
${ }^{2}$ The pseudo-rapidity is defined as a function of the polar angle: $\eta=-\log (\tan (\theta / 2))$.
} 


\begin{tabular}{|c|c|c|}
\hline estimator & description & cut value \\
\hline ETOT & cluster energy & $>5 \mathrm{GeV}$ \\
\hline NCEL & $\begin{array}{l}\text { number of cells assigned to the } \\
\text { electron candidate }\end{array}$ & $>3$ \\
\hline EAEM & $\begin{array}{l}\text { energy fraction in first } 2 \text { layers } \\
\text { of the electromagnetic section } \\
(3 \text { layers in IF) }\end{array}$ & $>0.94+0.05 \cos (2 \theta)$ \\
\hline EAHN & hot core fraction & $\begin{array}{l}>0.4 \text { in forward region } \\
>0.8 \text { in backward region }\end{array}$ \\
\hline EATR & transverse dispersion & $\begin{array}{l}<3 \text { in forward region } \\
<7.5 \text { in forward region }\end{array}$ \\
\hline EAIF & $\begin{array}{l}\text { fraction of the cluster energy to the } \\
\text { total energy within an isolation cone } \\
\text { with radius } \\
R=\sqrt{\left(\eta_{e}-\eta_{\text {cell }}\right)^{2}+\left(\phi_{e}-\phi_{\text {cell }}\right)^{2}}<0.25\end{array}$ & \multirow[t]{2}{*}{$\begin{array}{l}\text { EAIF }>0.95 \text { if } \\
\text { EAHD-ETOT }(1-E A E M)<300 \mathrm{MeV} \\
\text { EAIF }>0.98 \text { otherwise }\end{array}$} \\
\hline EAHD & hadronic energy in isolation cone & \\
\hline
\end{tabular}

Tab. 4.3: Estimators and cut values evaluated by QECFWD to identify electron candidates.

length of the track projection in the $x-y$ plane leads to a limited quality of the transverse momentum measurement.

For polar angles $\theta>25^{\circ}$ the acceptance of the Central Jet Chamber CJC2 is reached, improving the track measurement in the transition region $\left(20^{\circ}<\theta<37^{\circ}\right)$.

The central region $\left(37^{\circ}<\theta<140^{\circ}\right)$ is fully covered by both Central Jet Chambers CJC1 and CJC2. The radiation length of the dead material in this region is short and the track projection in the $x-y$ plane is developed to maximum length, providing a precise reconstruction and measurement of charged tracks.

\section{Cluster Criteria}

In the central region $\left(\theta>37^{\circ}\right)$ the fraction of the electron energy to the total energy within a cone of a radius $R=0.75$ in the pseudo-rapidity-azimuth $(\eta-\phi)$ plane around the electron direction must exceed $97.5 \%$. In the region not fully covered by the central drift chambers a tighter isolation is required by extending the cone radius to $R=1$.

The rejection power of the applied cuts has been studied using an inclusive electron sample in which all cuts have been applied one after another. Based on the preselection of the run period 1998-2000 (cf. section 4.2) events including at least one electron candidate with $P_{T}>20 \mathrm{GeV}$ located in the polar angle range $10^{\circ}<\theta<140^{\circ}$ are extracted.

Figure 4.2 presents the fraction of the electron energy in the isolation cone $R=0.75$ and $R=1.00$ for the electron candidate with the highest $P_{T}$, separately for the forward and central region. Shown are the distributions of the data and the dominantly contributing SM processes. The main contamination arises from photoproduction events, 

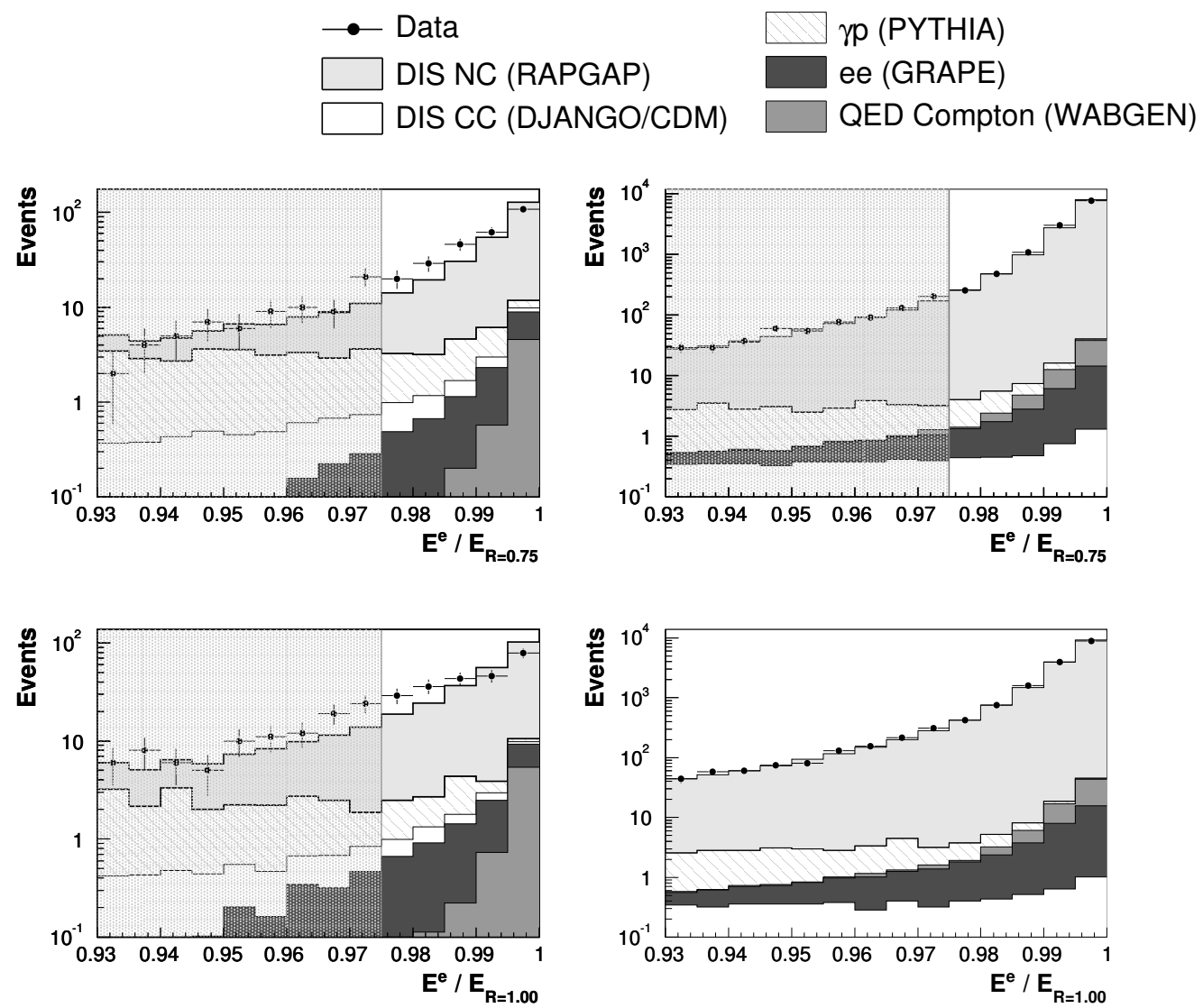

Fig. 4.2: The fraction of the electron energy in the isolation cone $R=0.75$ and $R=1.00$ for electron candidates in the forward (left side) and central (right side) region.

where hadrons are falsely identified as electron candidates. The rate of this contribution is efficiently reduced by the introduced cut.

\section{Track Criteria}

Another source for the misidentification of electrons are photons e.g. originating from $\pi^{0}$-decays. Since photons leave similar shower profiles in the electromagnetic section of the LAr calorimeter they cannot be distinguished from electrons on the basis of the cluster parameters. Therefore an isolated high quality track ${ }^{3}$ is required to match the electromagnetic cluster within a distance of closest approach $D C A_{c l}^{t r}<12 \mathrm{~cm}$. Tracks from the BOS [46] banks DTRA and DTNV are taken into account. While the DTRA bank contains only tracks fitted to the primary event vertex, tracks not associated with a vertex are included in the DTNV bank. No other good track is allowed around the electron direction within a distance $D_{t r}^{t r}<0.5$ in the $\eta-\phi$ plane.

Figure 4.3 presents the track-cluster distance $D C A_{c l}^{t r}$ and the distance between the cluster and the nearest good track $D_{t r}^{t r}$ for the inclusive electron sample. At this point the cluster

\footnotetext{
${ }^{3}$ The track selection is performed by the H1 software package QHQTRK [41, 45].
} 

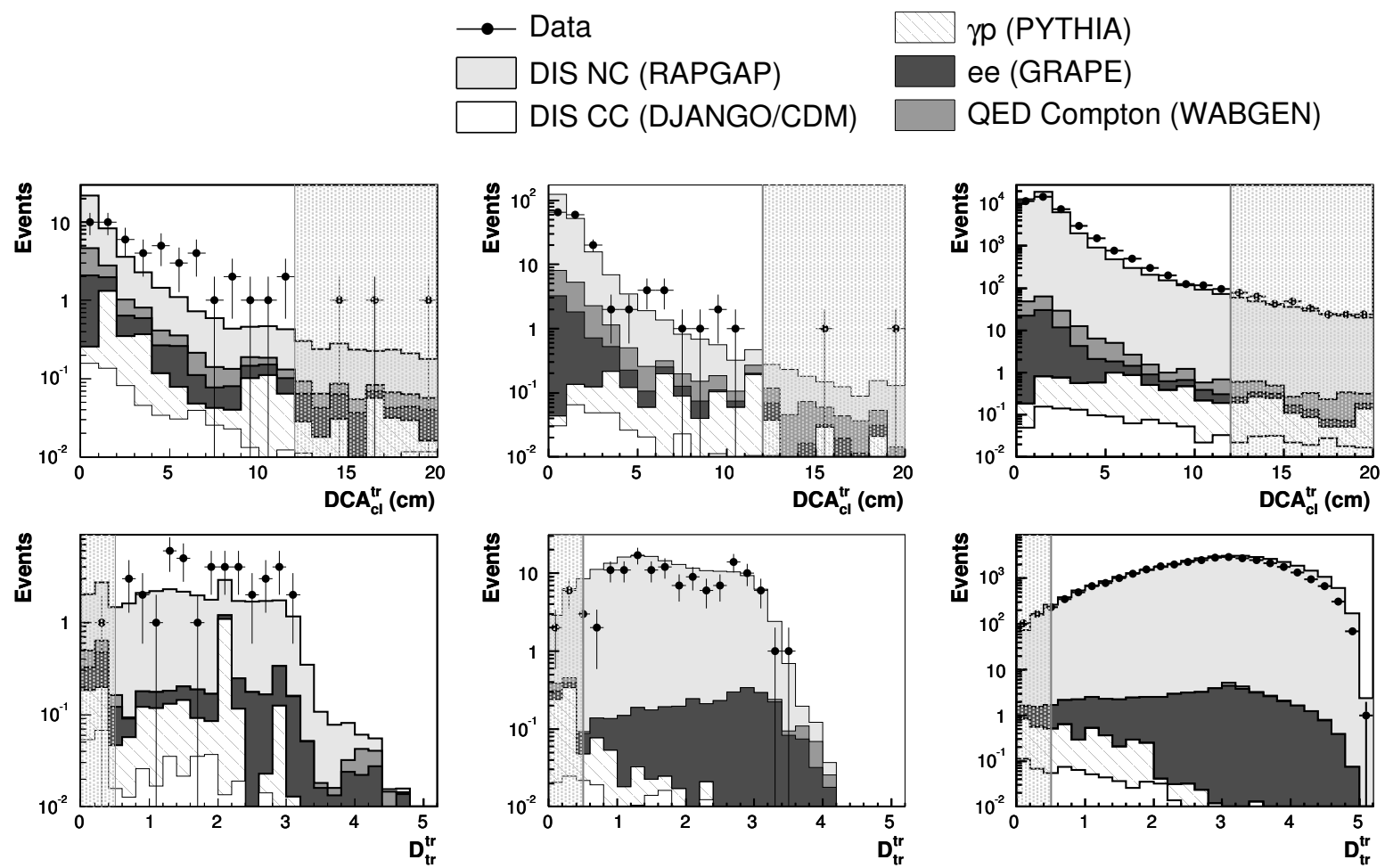

Fig. 4.3: The track cluster distance $D C A_{c l}^{t r}$ and the distance between the electron track and the nearest good track $D_{t r}^{t r}$ for electron candidates in the very forward (left side), transition (middle) and central (right side) region.

criteria are already required. The cuts are applied in all three polar angle regions to ensure an unambiguous association of a well isolated track to the electron cluster. It is obvious that the track isolation decreases in the forward region while the background contribution increases.

A remaining source of background arises from photons which convert to $e^{+} e^{-}$pairs. This reaction preferably takes place where the density of material is high, mainly degrading the electron identification in the forward region $\left(\theta<37^{\circ}\right)$. To suppress this kind of background, hits in either the inner or outer layer of the CIP are demanded for electron candidates within a distance of $\Delta z_{C I P}<10 \mathrm{~cm}$ to the extrapolated $z$-impact of the electromagnetic cluster to the CIP surface. The distance $\Delta z_{C I P}$ is given by

$$
\Delta z_{C I P}=\left(z_{c l}-z_{v t x}\right) \frac{R_{C I P}}{r_{c l}}-z_{v t x}+z_{C I P},
$$

where $z_{v t x}, z_{c l}$ and $r_{c l}$ denote the $z$ position of the event vertex, the $z$-coordinate of the electron cluster and the radial distance between the cluster and the beam axis. The fixed radii of the inner or outer layer of the CIP are given by $R_{C I P}$ while $z_{C I P}$ denotes the reconstructed $z$-coordinate of the nearest active CIP pad. Only active pads matching the azimuth angle of the electron cluster are considered, resulting in a resolution of $45^{\circ}$ (cf. section 2.2.2). 
In order to reject photons that convert late in the central tracker material the radial distance between the first measured point in the Central Jet Chambers and the beam axis $R_{\text {start }}$ is required to be below $30 \mathrm{~cm}$.

Figure 4.4 presents the discussed observables to reject background arising from photon conversion. The upper diagrams show the distributions of the $z$-distance for the outer and inner CIP layer, respectively, each under the condition that no hit within $\Delta z_{C I P}<10 \mathrm{~cm}$ has been reconstructed in the other layer. The figures indicate that most of the electrons produce hits in one of the CIP layers at small distances, while the background accumulates at larger values, mainly contributing in the forward region where the cut is applied. Further details on electron identification using the CIP can be found in [47].

The radial distance $R_{\text {start }}$ between the track and the beam axis is presented in the lower diagrams of figure 4.4. In the very forward region, only partially covered by the Central Jet Chambers, this quantity is not available.

The presented track requirements suppress the background arising from uncharged particles misidentified as electrons very efficiently. Nevertheless, the small fraction of background left nearly eliminates the identification of multi-electron events. It mainly originates from photons wrongly assigned to a track which satisfies the above criteria.

The fraction of those events can be reduced by the requirement, that the transverse momentum reconstructed from the associated track $P_{T}^{t r}$ matches the calorimetric measurement $P_{T}^{c l}$ of the electron cluster within $1 / P_{T}^{t r}-1 / P_{T}^{c l}<0.02 \mathrm{GeV}^{-1}$. To minimise systematic errors the reciprocal value of the transverse momentum is used, which depends directly proportional on the measured track curvature.

Figure 4.5 shows the distribution of the $P_{T}$-match value for the leading electron candidates of the inclusive sample. Since the quality of the transverse momentum measurement is limited in the very forward region the cut is only applied for polar angles $\theta>20^{\circ}$.

A summary of the electron identification criteria is given in table 4.4.

The rejection power of the cuts is corroborated in figure 4.6. Presented are the distributions of the transverse momentum $P_{T}$ and the polar angle $\theta$ of the first and second electron candidate of the inclusive electron sample before and after applying the identification criteria.

While the main background to the distributions of the leading electron candidate arises from photoproduction and CC interactions, the dominant contribution to the second candidate comes from NC and QED Compton events, where hadrons or photons are misidentified as electrons. After the application of the identification criteria the major rates in the distributions solely arise from signal processes, i.e. NC and QED Compton events for the leading electron, while the distributions of the second electron are strongly dominated by the electroweak production of di-electron pairs.

The selection efficiency for electrons is dominated by the track association, whose efficiency is determined in the following section. A quantitative specification of the efficiency and purity for various event configurations including electrons is given in section 5.5. 

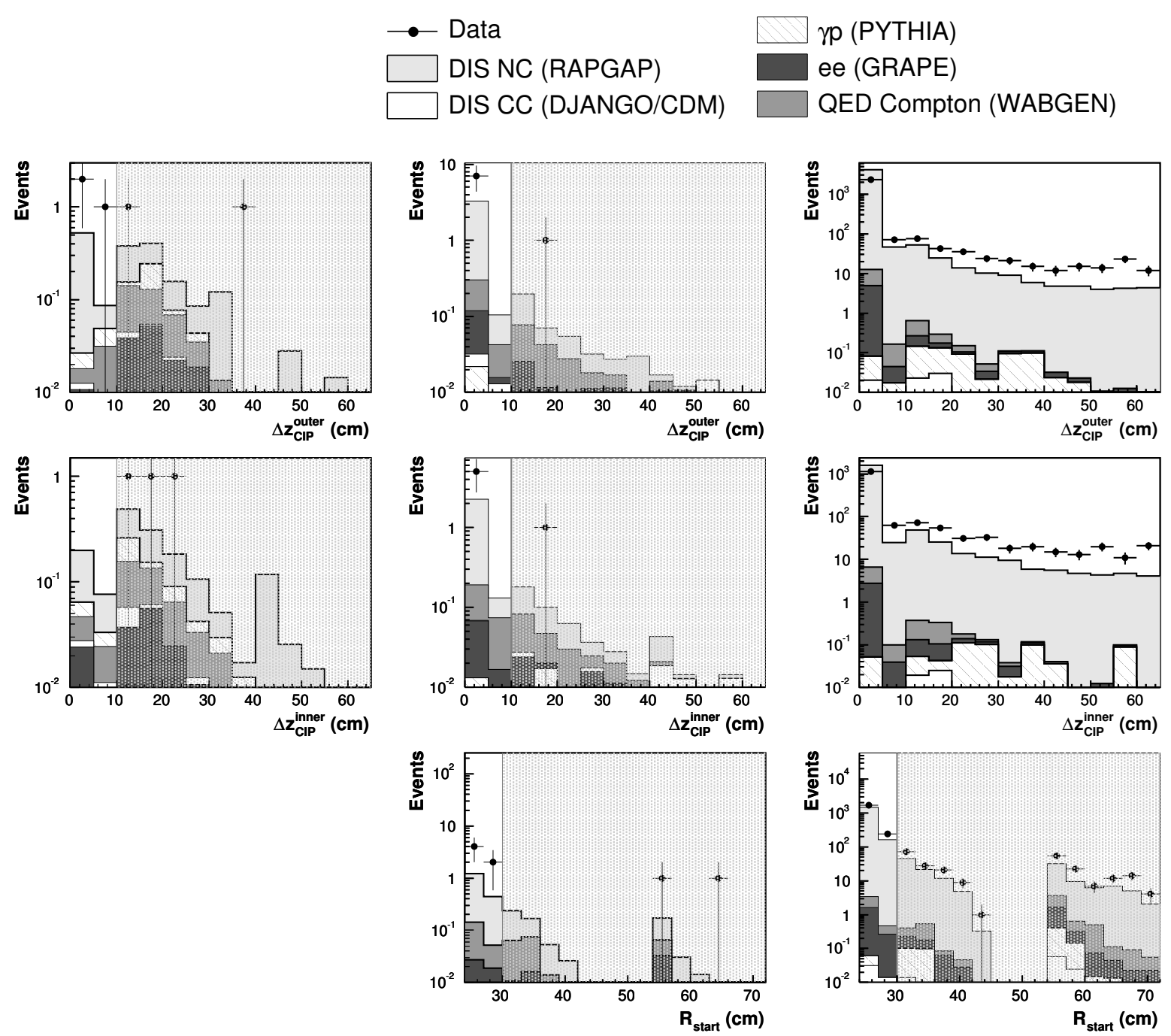

Fig. 4.4: The distance $\Delta z_{C I P}$ for the outer and inner CIP layer and the radial distance $R_{\text {start }}$ between the track and the beam axis for electron candidates in the very forward (left side), transition (middle) and central (right side) region.
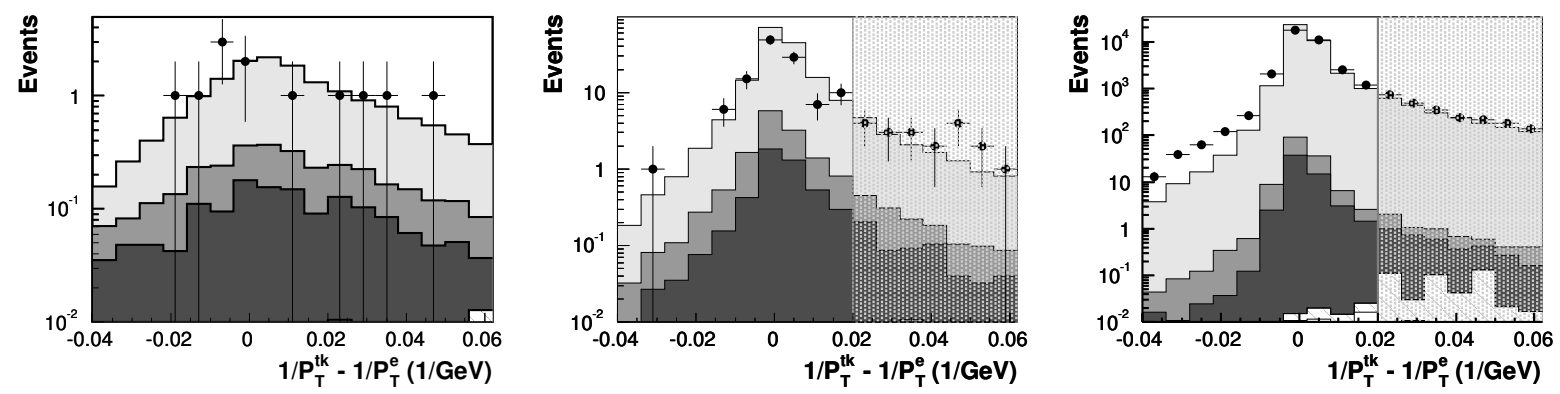

Fig. 4.5: The $P_{T}$-match value for electron candidates in the very forward (left side), transition (middle) and central (right side) region. 

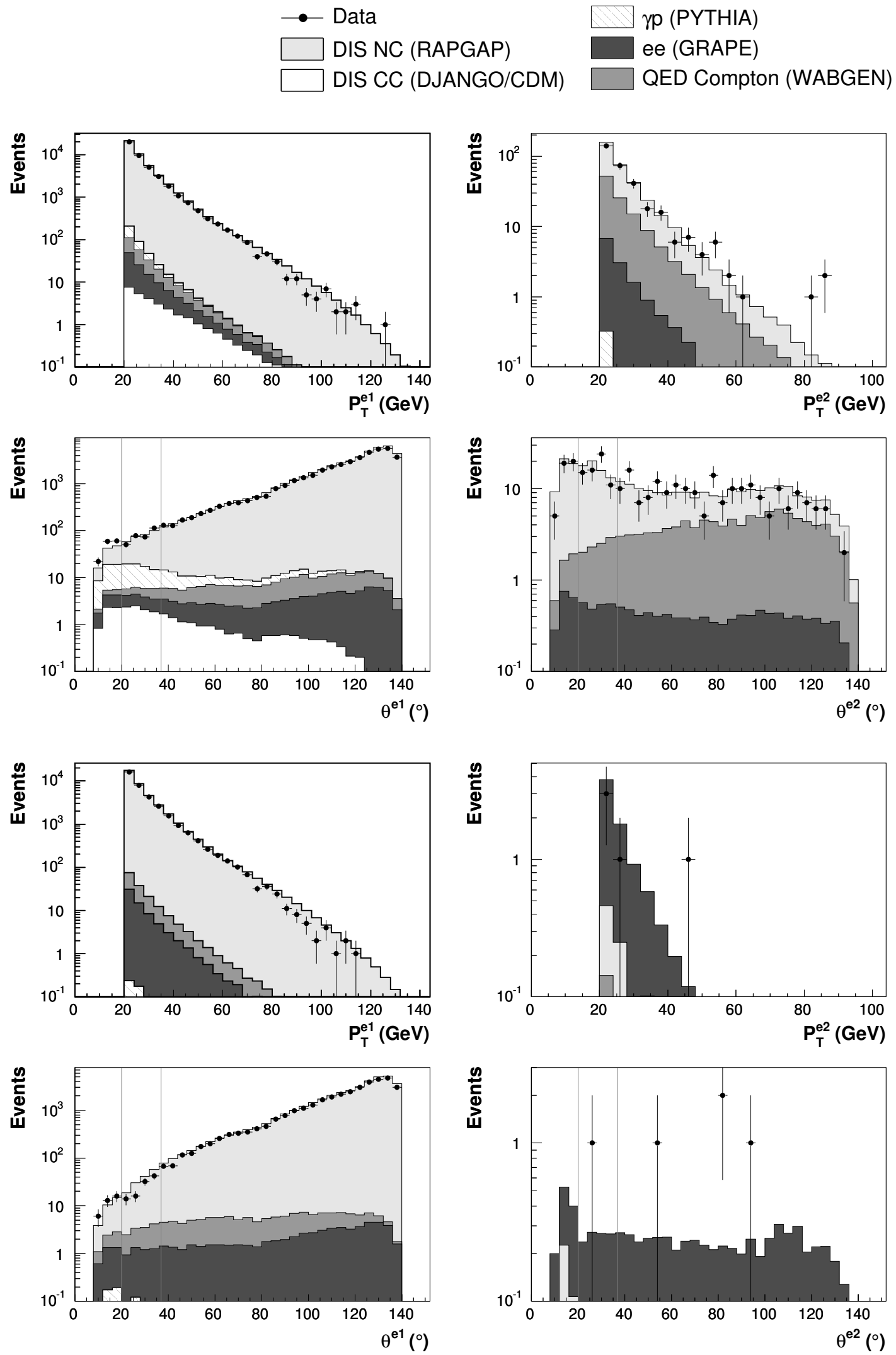

Fig. 4.6: The $P_{T}$ and $\theta$ distributions for the first (left side) and second (right side) electron candidate before (upper figures) and after (lower figures) applying the electron identification criteria. The vertical lines in the $\theta$ distributions indicate the three different polar angle regions. 


\begin{tabular}{|c|c|}
\hline \multicolumn{2}{|l|}{ phase space } \\
\hline \multicolumn{2}{|l|}{ acceptance } \\
\hline \multicolumn{2}{|c|}{$\begin{array}{l}z>-180 \mathrm{~cm} \text { and } z \notin[-70,-65] \mathrm{cm} \text { and } z \notin[15,25] \mathrm{cm} \\
\left|\phi-\phi_{\text {crack }}\right|>2^{\circ} \text { with } \phi_{\text {crack }} \in\left[0^{\circ}, 45^{\circ}, 90^{\circ}, 135^{\circ}, 180^{\circ}, 225^{\circ}, 270^{\circ}, 315^{\circ}\right] \\
\phi \notin\left[230^{\circ}, 250^{\circ}\right] \text { for } \text { run } \in[177920,200407] \\
\phi \notin\left[190^{\circ}, 280^{\circ}\right] \text { for } \text { run } \in[257590,261338]\end{array}$} \\
\hline \multicolumn{2}{|c|}{ electron cluster criteria } \\
\hline $\begin{array}{l}10^{\circ}<\theta<37^{\circ} \\
37^{\circ}<\theta<140^{\circ}\end{array}$ & $\begin{array}{l}E_{R=1.00} / E^{e} \leq 2.5 \% \\
E_{R=0.75} / E^{e} \leq 2.5 \%\end{array}$ \\
\hline \multicolumn{2}{|c|}{ electron track criteria } \\
\hline $37^{\circ}<\theta<140^{\circ}$ & $\begin{array}{l}D C A_{c l}^{t r}<12 \mathrm{~cm} \quad \text { and } \quad D_{t r}^{t r}>0.5 \\
R_{\text {start }}<30 \mathrm{~cm} \\
1 / P_{T}^{t r}-1 / P_{T}^{e}<0.02\end{array}$ \\
\hline $20^{\circ}<\theta<37^{\circ}$ & $\begin{array}{l}D C A_{c l}^{t r}<12 \mathrm{~cm} \quad \text { and } \quad D_{t r}^{t r}>0.5 \\
R_{\text {start }}<30 \mathrm{~cm} \\
1 / P_{T}^{t r}-1 / P_{T}^{e}<0.02 \\
\Delta z_{C I P}<10 \mathrm{~cm} \quad \text { and } \quad \Phi_{C I P}<45^{\circ}\end{array}$ \\
\hline $10^{\circ}<\theta<20^{\circ}$ & $\begin{array}{l}D C A_{c l}^{t r}<12 \mathrm{~cm} \text { and } D_{t r}^{t r}>0.5 \\
\Delta z_{C I P}<10 \mathrm{~cm} \text { and } \Phi_{C I P}<45^{\circ}\end{array}$ \\
\hline
\end{tabular}

Tab. 4.4: Electron identification criteria.

\section{Electron Track Association Efficiency}

The efficiency of the track association to the electron cluster plays a decisive role within the scope of electron identification, since it dominates the total electron finding efficiency (cf. section 5.5). Furthermore it must be ensured that the simulation precisely describes the efficiency obtained from the data.

In order to determine the track association efficiency depending on the electron track criteria a clean NC event sample is selected. Based on the preselection presented in section 4.2 events including at least one electron candidate with $P_{T}>20 \mathrm{GeV}$ in the polar angle range $10^{\circ}<\theta<140^{\circ}$ are selected by applying only the cluster criteria. The events are required to be well balanced in longitudinal as well as transverse momentum,

$$
45 \mathrm{GeV}<\sum_{i}\left(E_{i}-P_{z, i}\right)<65 \mathrm{GeV} \text { and } 0.6<P_{T}^{\text {had }} / P_{T}^{e}<1.2,
$$

in order to suppress non-ep and photoproduction background. Furthermore the inelasticity $y_{e}$ is restricted to values

$$
y_{e}<0.9 \text {. }
$$

Background arising from QED Compton interactions is suppressed by rejecting events with more than one electron candidate. 

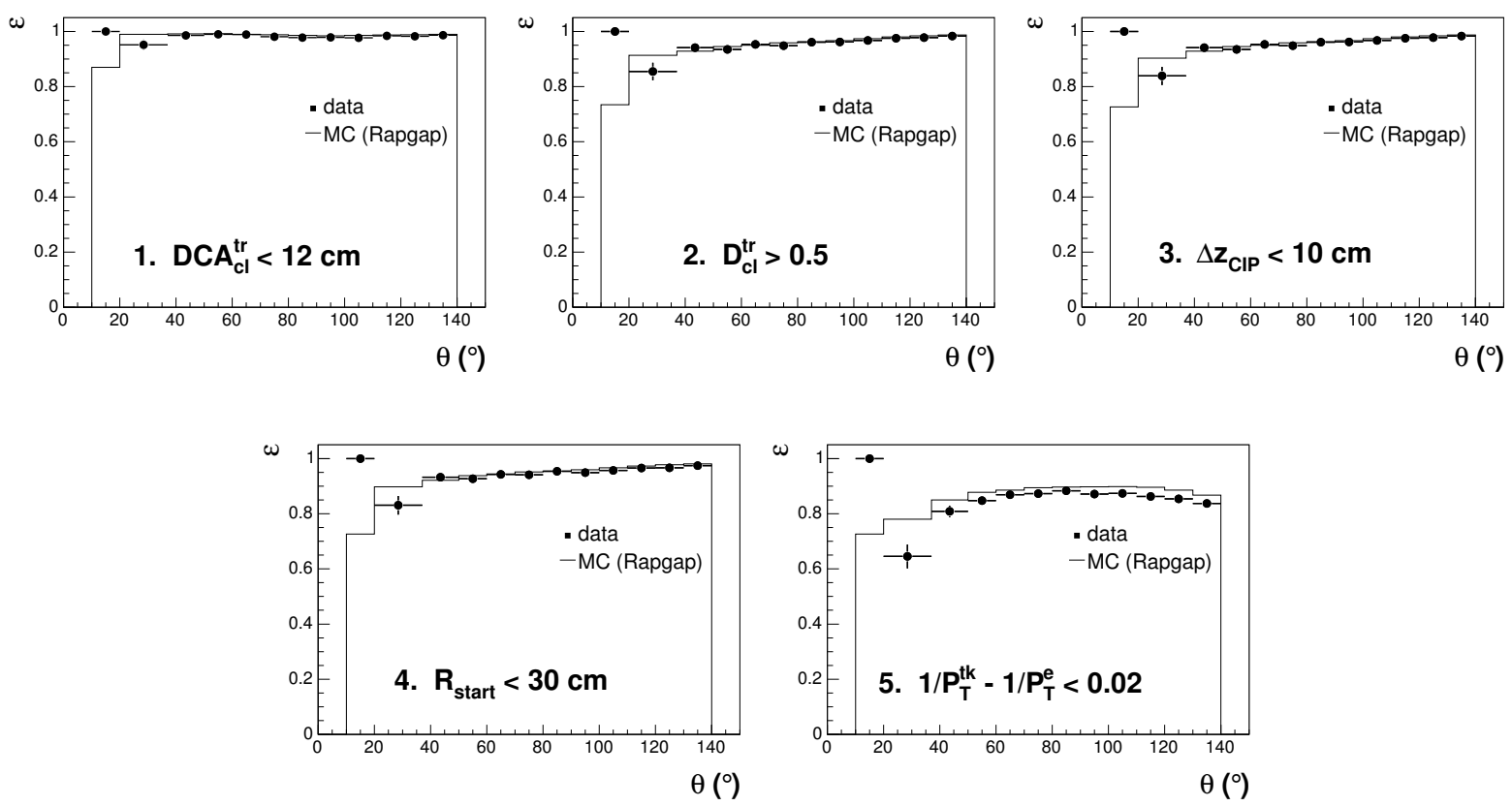

Fig. 4.7: The electron track association efficiency as a function of the polar angle $\theta$ depending on the electron track criteria.

Figure 4.7 presents the electron track association efficiency as a function of the polar angle $\theta$. The efficiency is determined in dependence of the electron track criteria applied one after another.

Starting from values close to $100 \%$ in case of only demanding a track linked to the cluster (fig. 4.7 (1)), the efficiency decreases stepwise to approximately $85 \%$ in the central region and $65 \%$ in the forward region ${ }^{4}$ (fig. $4.7(5)$ ). It is incidental that the $P_{T}$-match requirement contributes most to the decrease of efficiency, but since the cut is essential for the identification of multi-electron events it must be compromised.

The efficiency predicted by the simulation slightly overshoots the final value determined by data. Therefore the Monte Carlo has been re-weighted by artificially removing tracks within the electron identification. A re-weighting factor of $2 \%$ is applied for the run period 1994-1997, while 4\% of associated Monte Carlo tracks are removed in 1998-2000. To cope with the uncertainty of the re-weighting procedure absolute errors of $2 \%$ and $7 \%$ are considered for the track association efficiency in the central and forward region, respectively. It is obvious that this efficiency correction also affects the photon identification, since electrons without an associated track convert to further photon candidates (cf. section 4.3.2).

Figure 4.8 presents the finally obtained track association efficiency as a function of the transverse momentum $P_{T}$ and the polar angle $\theta$ of the electron. The values predicted by

\footnotetext{
${ }^{4}$ Since the determination of the efficiency in the very forward region $\left(\theta<20^{\circ}\right)$ is based on only a single event the value predicted by data is not reliable in this bin.
} 

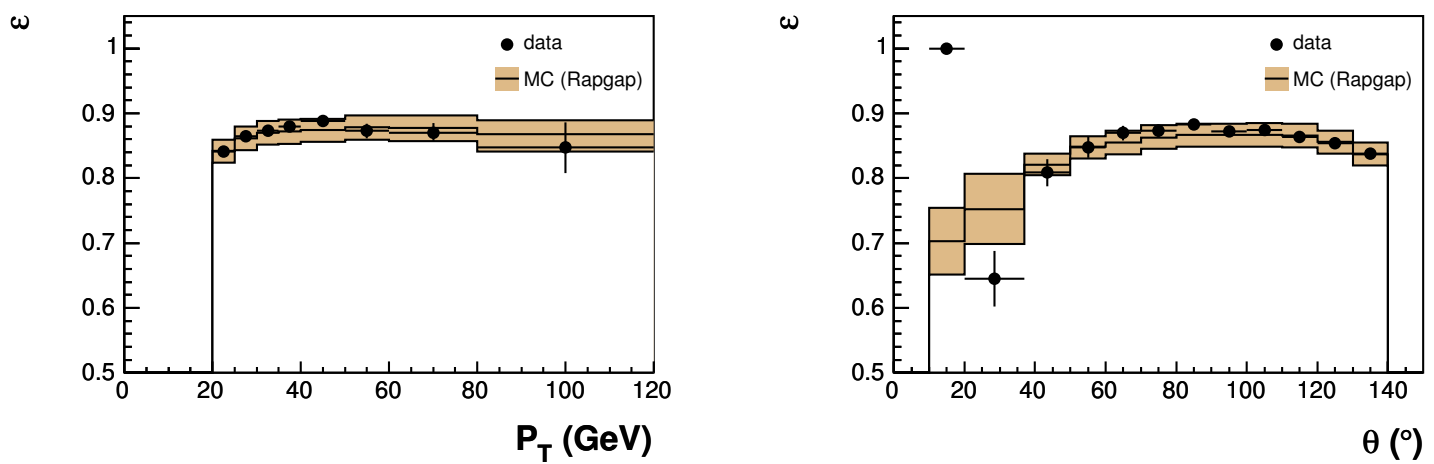

Fig. 4.8: The final electron track association efficiency as a function of the transverse momentum $P_{T}$ (left side) and the polar angle $\theta$ (right side).

the data are well described by the simulation within the considered systematic uncertainty.

\subsubsection{Photons}

The photon identification is based on the same measurement of a compact and isolated electromagnetic shower as the electron identification. Identical cluster criteria are applied on the objects selected by QECFWD to suppress hadronic background. To explicitly avoid misidentifications of photons originating from jets a minimum distance of $R>1$ in the $\eta-\phi$ plane is required between the direction of the photon candidate and any jet with $P_{T}>5 \mathrm{GeV}$. The same acceptance requirements are demanded to ensure an unambiguous identification of photon candidates.

In order to distinguish between photons and charged particles vetoes on any track pointing to the electromagnetic cluster are applied. Neither DTRA nor DTNV tracks are allowed around the photon direction within a distance $D_{c l}^{t r}<0.5$. Additionally the distance of closest approach $D C A_{c l}^{t r}$ between any track and the electromagnetic cluster must exceed the value of $24 \mathrm{~cm}$.

A veto on hits in any layer of the CIP within a distance $\Delta z_{C I P}$ to the extrapolated $z$-impact of the electromagnetic cluster to the CIP surface is ideally suited to reject misidentified electrons, actually the largest background contribution. Since the CIP is operated independently a hit veto accounts for inefficiencies of the Central Jet Chambers. The CIP requirement gets vitally important in the forward region where the acceptance of the CJC is limited.

The rejection power of the applied photon identification criteria is studied in the same manner as for the electron cuts. Based on the preselected inclusive electron event sample (cf. section 4.3.1) the photon identification criteria are applied one after another. Figure 4.9 presents the distributions of the corresponding observables.

Starting from the initial sample, selected by the application of the cluster criteria only, 

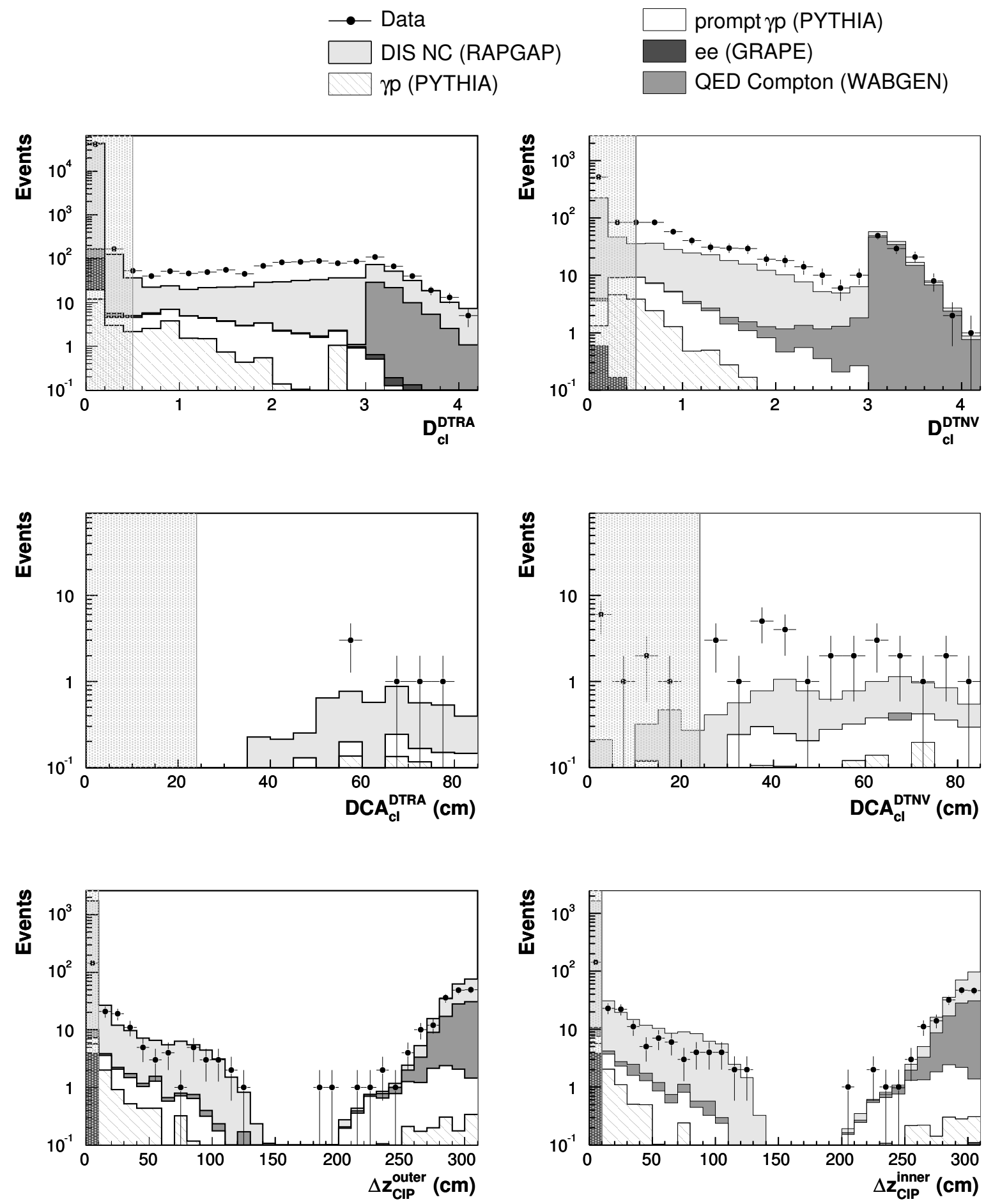

Fig. 4.9: The distances between the photon cluster and the nearest tracks $D_{c l}^{t r}$, the track cluster distance $D C A_{c l}^{t r}$ and the distance $\Delta z_{C I P}$ for the outer and inner CIP layer for photon candidates of an inclusive event sample. 


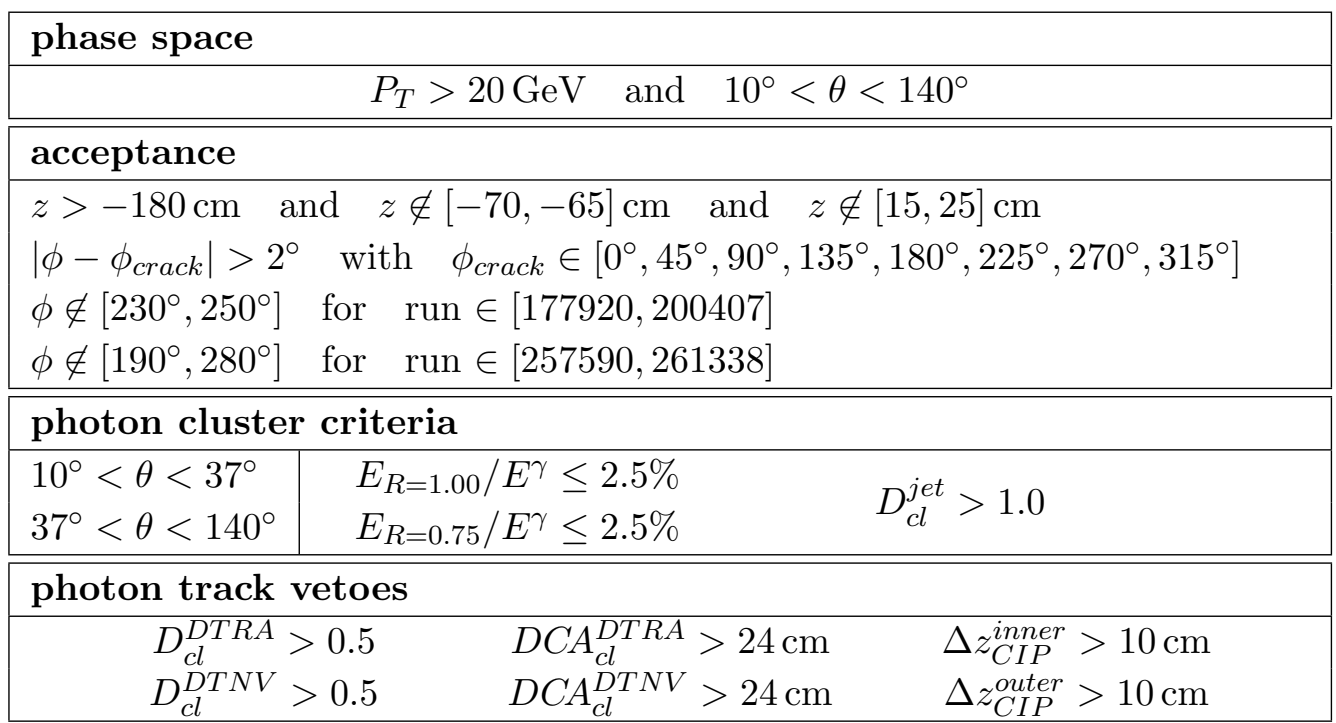

Tab. 4.5: Photon identification criteria.

background arising from $\mathrm{NC}$ interactions contributes dominantly to the distributions of the track-cluster distances $D_{c l}^{D T R A}$ and $D_{c l}^{D T N V}$. These background events accumulate at small values while signal events from prompt photon production and QED Compton interactions populate the region of larger distances. Since the radiation of real photons is accounted for within the event generator RAPGAP, a fraction of neutral current DIS events contributes to the photon signal, too ${ }^{5}$.

After the isolation of the electromagnetic cluster from tracks in the $\eta-\phi$ plane only a small fraction of tracks geometrically matches the electromagnetic cluster within a distance of closest approach $D C A_{c l}^{t r}<24 \mathrm{~cm}$. In most of the events even no track is pointing close to the cluster centre-of-gravity, which are then not included in the presented distributions of $D C A_{c l}^{D T R A}$ and $D C A_{c l}^{D T N V}$.

The distributions of the $z$-distance for the outer and inner CIP layer illustrate the expected behaviour of charged and uncharged particles crossing the CIP. While charged particles produce hits in pads close to the point of impact at the CIP surface, naturally no activity is noticed for uncharged particles as photons. If so, the $\Delta z_{C I P}$-distribution reflects the distribution of the $z$-coordinate of the event vertex centred around the default value of $300 \mathrm{~cm}$. The requirement $\Delta z_{C I P}>10 \mathrm{~cm}$ for both, the inner and outer layer of the CIP, efficiently reduces the contribution of misidentified electrons.

A summary of the photon identification criteria is given in table 4.5. Further details on the photon identification can be found in [47].

The quality of the applied cuts is demonstrated in figure 4.10. Presented are the trans-

\footnotetext{
${ }^{5}$ Since the signal and background contributions within the NC DIS sample are not separated, the plots presented in this section may just give a first impression of the quality of the applied cuts. The quantitative examination of the identification efficiencies and purities for all event classes investigated in this analysis is undertaken in section 5.5.
} 

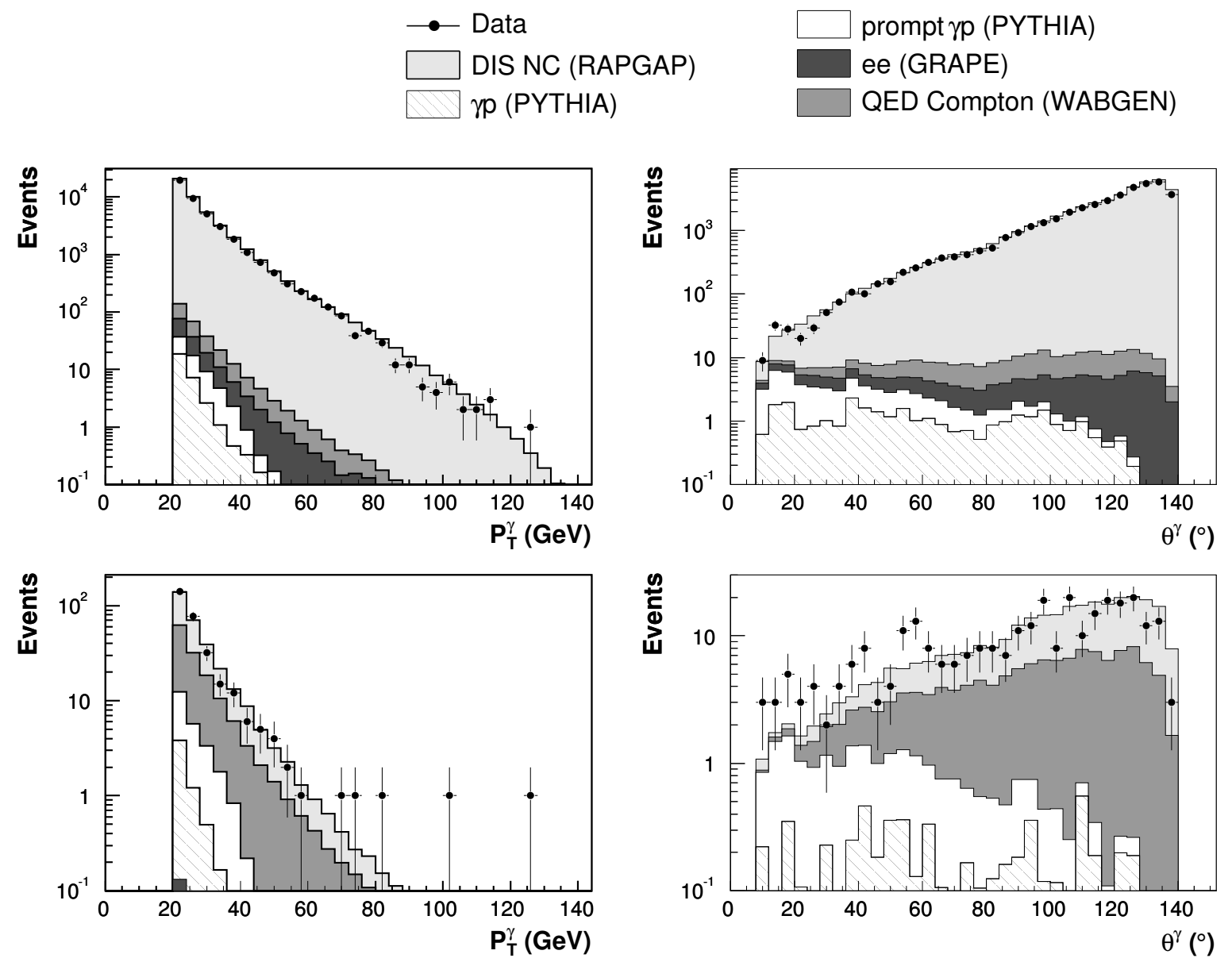

Fig. 4.10: The $P_{T}$ and $\theta$ distributions of the photon candidate before (upper figures) and after (lower figures) applying the identification criteria.

verse momentum $P_{T}$ and the polar angle $\theta$ distributions of the selected photon candidates before and after the implementation of the identification criteria.

While the initial event sample is strongly dominated by misidentified electrons from NC interactions, the rate of photons originating from signal processes as prompt photon production, QED Compton processes and the emission of real photons from electrons outweighs the background contributions.

\section{Photon Identification Efficiencies}

The Central Inner Proportional chamber CIP is used in this analysis to distinguish charged and uncharged particles. It plays a decisive role within both the electron and photon identification. Therefore a careful investigation of the CIP efficiency is essential. Furthermore the misidentification probability for electrons strongly affects the photon identification. Both quantities are studied based on the clean sample of NC events introduced in section 4.3.1 and will be discussed in the following paragraphs. 

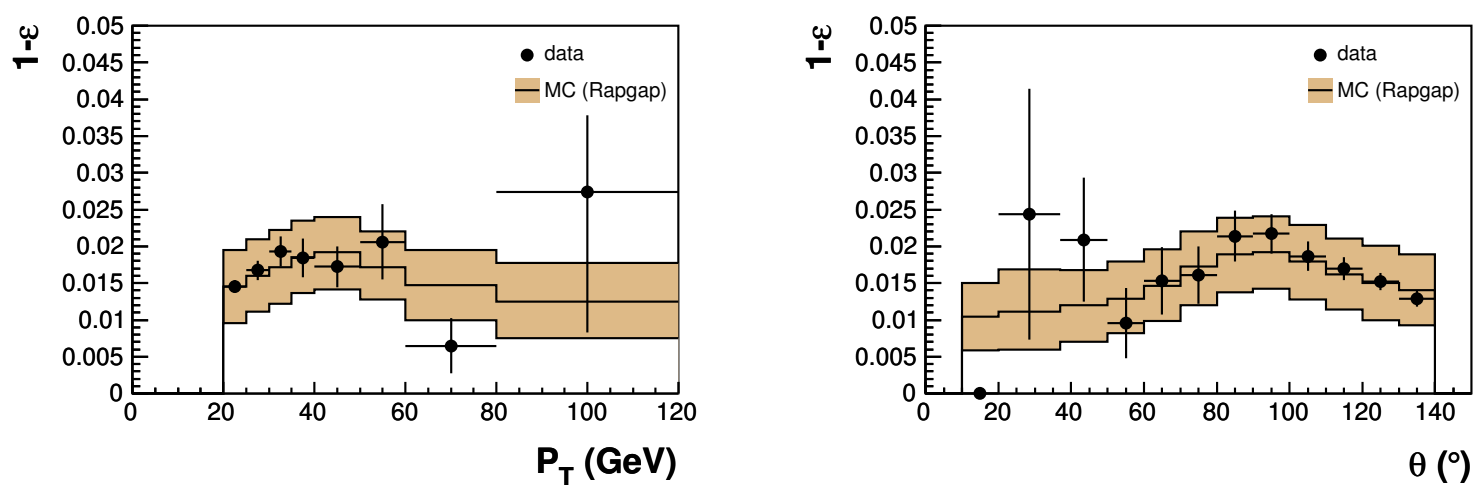

Fig. 4.11: The CIP inefficiency as a function of the transverse momentum $P_{T}$ and the polar angle $\theta$ of the electron

Efficiency of the CIP The efficiency of the CIP can be determined from data by the ratio of the number of electrons, which pass all track selection criteria, to the number of electrons identified without consideration of the CIP requirement. The values obtained by this method are satisfactorily high, ranging between $98 \%$ and $99 \%$ slightly depending on the transverse momentum and the polar angle of the electron.

It turned out that the simulation predicts an slight overshoot at this level of precision. Therefore the Monte Carlo has been re-weighted by artifically removing hits from the CIP for $1 \%$ of the electron candidates. An absolute error of $0.05 \%$ is applied on the hit efficiency of the CIP.

Figure 4.11 presents the distributions of the finally obtained CIP efficiency as a function of the transverse momentum $P_{T}$ and the polar angle $\theta$ of the electron. Shown are the difference of the obtained values to unity, representing the inefficiency of the CIP. The measured and simulated values agree well within the systematic error quoted.

Electron Misidentification Probability The misidentification probability of electrons is of particular importance for the photon identification since all unidentified electrons convert to further photon candidates. Due to the large cross section of the neutral current scattering process already small inefficiencies within the electron identification may eliminate the measurement of photon events. A precise description of the simulation must be guaranteed to ensure a non-ambiguous description of the photon background by the Monte Carlo. Since the Central Jet Chambers as well as the CIP are involved in the electron identification, hardware inefficiencies of each sub-detector may contribute.

Based on the inclusive event sample the electron misidentification probability is determined by the ratio of the number of electrons misidentified as photons to the total number of electron candidates selected by the cluster criteria only. Figure 4.12 presents the obtained results. As expected the statistics from data are low.

The upper diagrams show the misidentification probability as a function of the transverse 

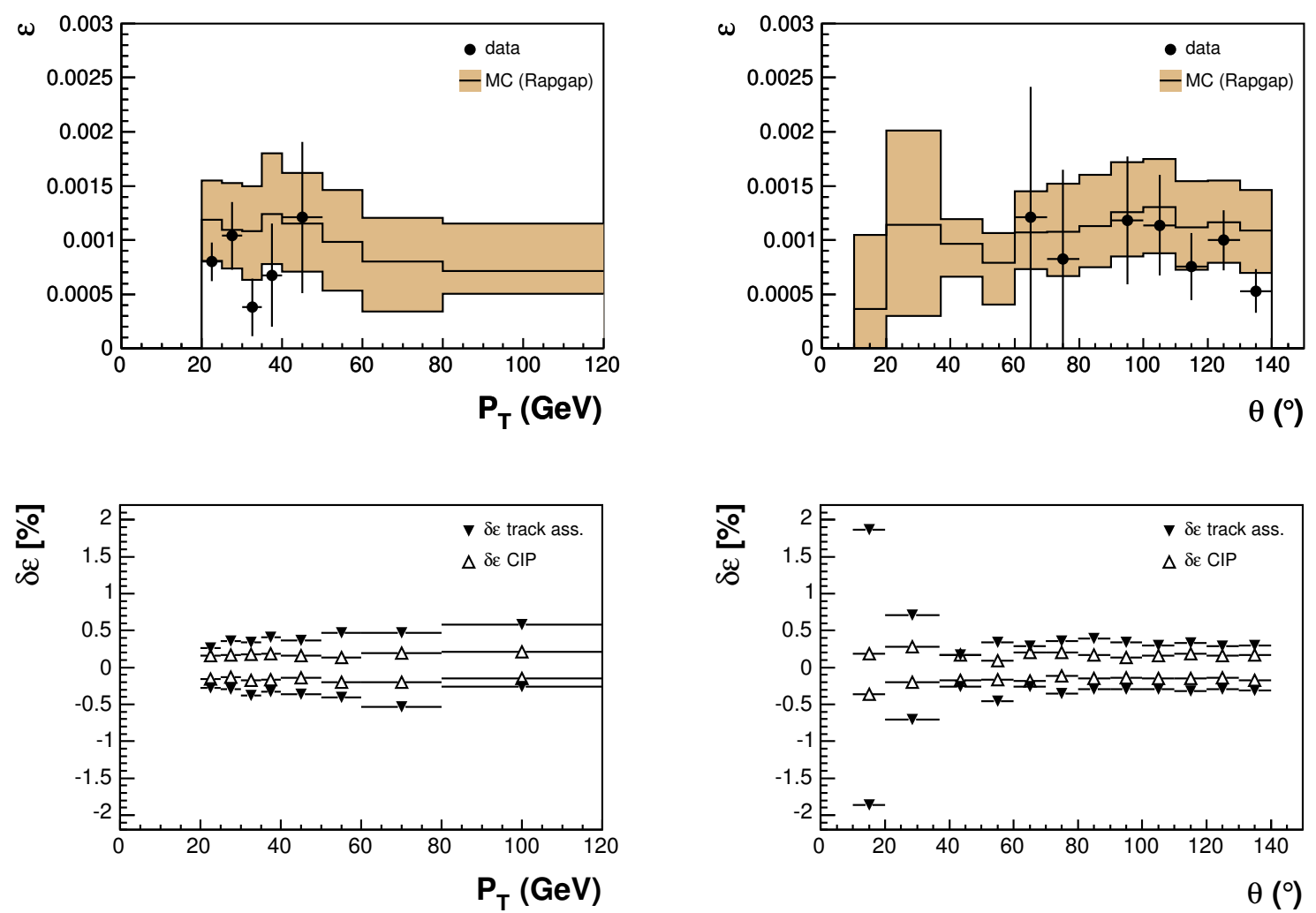

Fig. 4.12: The electron misidentification probability as a function of the transverse momentum $P_{T}$ and the polar angle $\theta$ (upper figures) and the contributing systematic errors of the electron track association and the CIP efficiencies (lower figures).

momentum $P_{T}$ and the polar angle $\theta$ of the electron. Only in the order of $0.1 \%$ of electron candidates are misidentified as photons. The derived values from data are well described by the simulation within the systematic uncertainties considered for the electron track association and CIP efficiencies. Both contributing errors are presented separately in the lower diagrams of figure 4.12. The total systematic uncertainty is derived by addition in quadrature.

\subsubsection{Muons}

This section briefly summarises the muon identification. A detailed description of further studies can be found in [41].

The muon identification is based on the reconstruction of a charged track in the inner tracking systems associated with either a track in the Forward Muon Detector or a track segment in the Instrumented Iron or some energy deposit in the Tail Catcher calorimeter. In the region of small polar angles $\theta<12.5^{\circ}$ muon candidates not linked to a central track are selected in the Forward Muon Detector, too. 


\begin{tabular}{|c|}
\hline phase space \\
\hline$P_{T}>20 \mathrm{GeV}$ and $10^{\circ}<\theta<140^{\circ}$ \\
\hline \hline isolation \\
\hline$D_{\mu}^{\text {jet }}>1 \quad D_{\mu}^{t r}>1 \quad E_{R=0.5}^{L A r}<8 \mathrm{GeV}$ \\
\hline \hline cosmic and beam halo background rejection cuts \\
\hline
\end{tabular}

Tab. 4.6: Muon identification criteria.

The muon momentum is measured by the track curvature in the toroidal or solenoidal magnetic field. The track selection in the inner tracking system is performed by the $\mathrm{H} 1$ software package QHQTRK and based on standard quality requirements [45]. Further basic muon identification criteria depend on the involved sub-detectors and the type of the muon candidate.

Forward Muon Detector Tracks reconstructed in the Forward Muon Detector must fulfil a certain track quality and the $z$ coordinate of the initial point is restricted to the first drift chamber layers.

Instrumented Iron The extrapolation of track segments reconstructed in the Instrumented Iron is required to geometrically match the $z$-coordinate of the event vertex within a certain distance. Furthermore a minimum number of streamer tube layers must have been activated.

Tail Catcher In order to suppress muon misidentification arising from hadronic showers leaking into the Tail Catcher, a minimum depth of LAr energy deposited in a cylinder centred on the muon direction is required. In addition at least one LAr cluster must contribute to the measurement.

After the preselection of muon candidates in the considered phase space harsher identification criteria are applied to ensure an unambiguous identification.

The main background results from muons originating from cosmic radiation. It dominantly contributes in multi-muon events and can efficiently be suppressed on the basis of topological event criteria, such as collinearity and timing conditions [42].

In di-muon events the track opening angle $\alpha$ is restricted to values below $165^{\circ}$. For large opening angles $\alpha>150^{\circ}$ additionally the polar angle sum $\theta_{\mu 1}+\theta_{\mu 2}$ of the muon-pair must be centred around $180^{\circ}$ within $10^{\circ}$.

Furthermore stronger timing requirements are demanded. The event timing (cf. section 4.2) for muon events is restricted to values $\left|\Delta T_{0}\right|<4.8 \mathrm{~ns}$ and an upper threshold of $3.8 \mathrm{~ns}$ is applied on the track timing difference between track segments in the upper and lower part of the Central Muon Detector. 


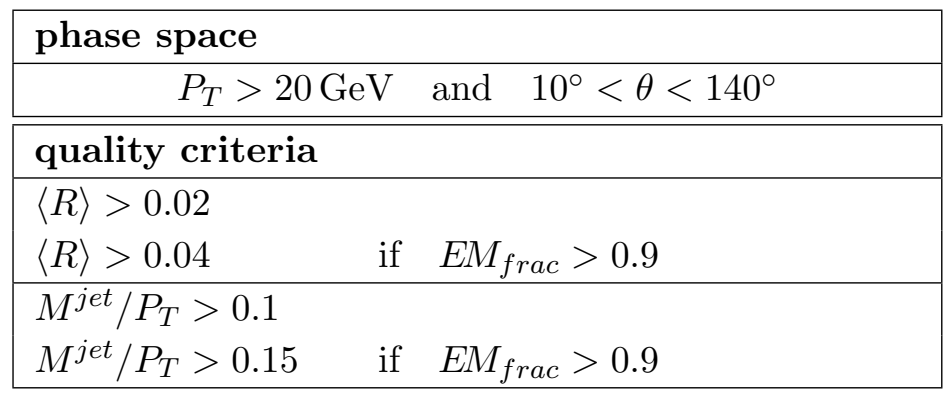

Tab. 4.7: Jet identification criteria.

The remaining background from cosmic muons is rejected by visual scanning.

Another background contribution arises from the misidentification of hadrons. Especially pions and kaons may either enter the Central Muon Detector or decay collinear in a muon in the inner tracking system. Finally energy leaking out of the LAr calorimeter may fake muon candidates.

In order to suppress the hadronic background tight isolation criteria are applied. No other good track is allowed around the muon direction within a distance of $D_{\mu}^{t r}<0.5$ in the $\eta-\phi$ plane. The deposit in the LAr calorimeter within a cylinder of radius $R=0.5$, centred on the muon direction, is restricted to energies smaller than $8 \mathrm{GeV}$. Furthermore muon candidates must be separated from the closest jet with $P_{T}>5 \mathrm{GeV}$ by $R>1$ unit in the $\eta-\phi$ plane.

The muon identification is completed by the rejection of beam halo events. Since beam halos typically transverse the detector parallel to the $z$-axis they are suppressed by the requirement of muon candidates having to originate from the event vertex.

The muon identification criteria are summarised in table 4.6.

\subsubsection{Jets}

This section briefly summarises the jet identification. A detailed description presenting further studies can be found in [41].

Jet candidates are defined using the inclusive $k_{\perp}$ algorithm $[48,49]$. The algorithm is applied in the laboratory frame with a separation parameter of 1 within a $P_{T}$ weighted recombination scheme in which the jets are treated as massless. The jet algorithm is run on all energy deposits not previously identified as electron or photon candidates.

Subsequent to the identification of jet candidates by the jet algorithm cuts are applied to adjust the background contribution. Although the inefficiencies of the electron search algorithm QECFWD are small (cf. section 4.3.1), the scattered electron may fake or be part of identified jets, essentially in regions with sizable amount of dead material or limited acceptance of the LAr calorimeter. The background rejection of misidentified electrons is 

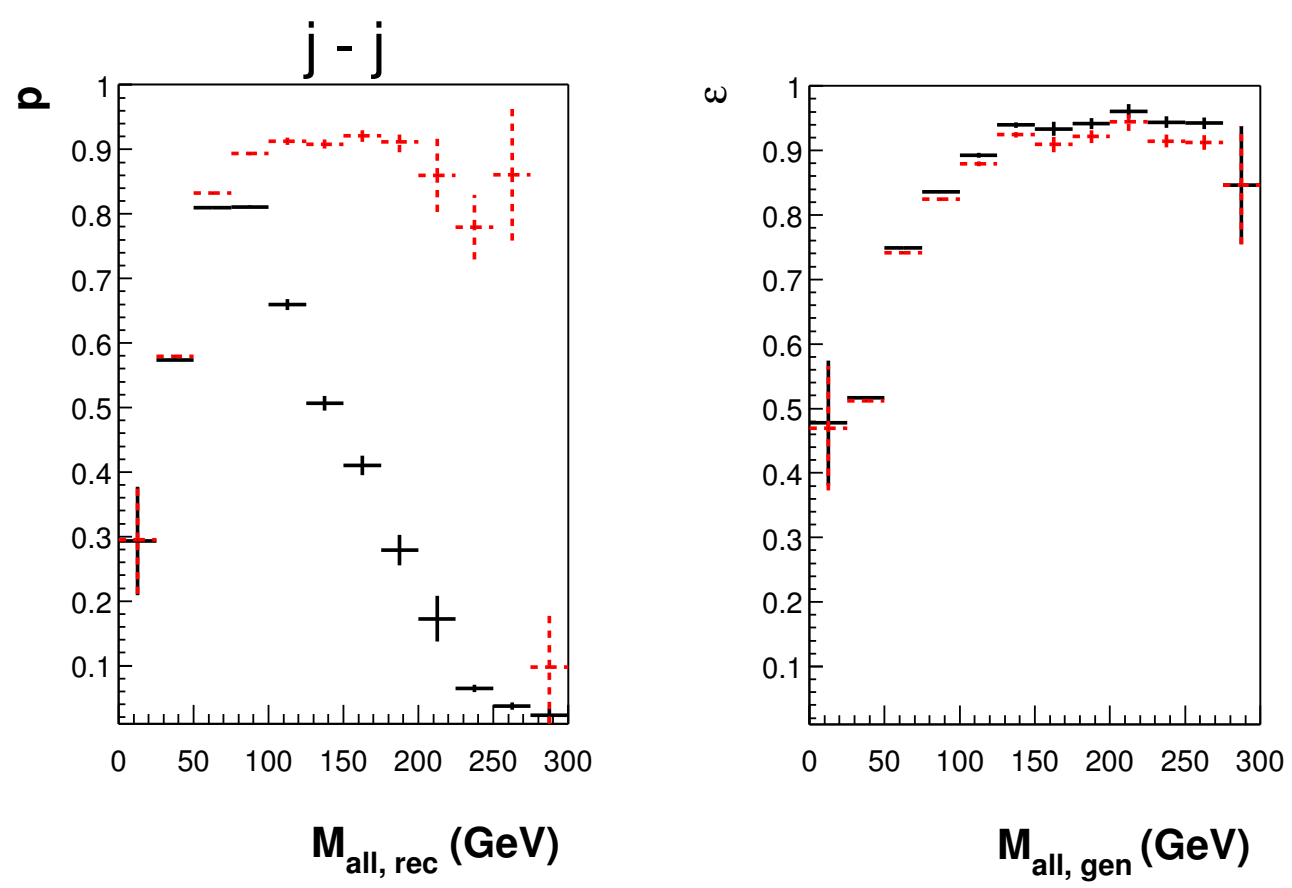

Fig. 4.13: Purity (left side) and efficiency (right side) before (solid line) and after (dashed line) the application of the jet identification criteria as a function of $M_{\text {all }}$ of the selected jets in the $j$ - $j$ event class. The subscripts rec and gen denote the reconstructed and generated quantity [41].

of particular importance, since it mainly contributes to regions of high transverse momenta or invariant jet masses in multi-jet topologies.

Extensive studies have been undertaken in order to suppress the rate of misidentified electrons [41]. Since jets faked by electrons are mostly very collimated and dominated by energy deposits in the electromagnetic section of the LAr calorimeter, jet candidates are required to have a radial moment greater than 0.02 . In case of very high electromagnetic fractions $E M_{\text {frac }}>0.9$ the threshold of the cut is raised to 0.04 . The radial moment of a jet is given by

$$
\langle R\rangle=\frac{\sum_{i} P_{T, i} R_{i}}{\sum_{i} P_{T, i}}
$$

where the sum runs over all objects belonging to the jet and $R_{i}$ denotes the distance between the individual objects and the jet axis in the $\eta-\phi$ plane [19].

The ratio between the invariant mass of the jet $M^{\text {jet }}$ and its transverse momentum $P_{T}$ must exceed a value of 0.1 . In the same way as for the applied condition on the radial moment the cut is tightened to 0.15 , if the fraction of jet energy contained in the electromagnetic section of the LAr calorimeter is greater than 0.9. The invariant mass $M^{\text {jet }}$ is derived by

$$
M^{\text {jet }}=\sqrt{\left(\sum_{i} p_{i}\right)^{2}},
$$

where the sum runs over the four-momenta $p_{i}$ of all objects belonging to the jet. 


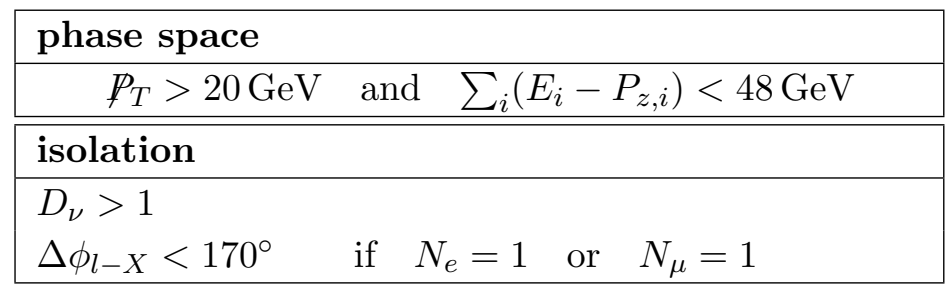

Tab. 4.8: Neutrino identification criteria.

The rejection power of the applied cuts is illustrated in figure 4.13. It shows the purity and efficiency before and after the application of the jet identification criteria as a function of the invariant mass $M_{\text {all }}$ of the selected jets in events with exactly two jets. The purity (efficiency) is defined as the ratio of SM events with two generated as well as reconstructed jets to the total number of events with exactly two reconstructed (generated) jets (cf. section 5.5). About $85 \%$ of non-genuine jets but only $3 \%$ of the genuine jets are rejected by the applied requirements.

The jet identification criteria are summarised in table 4.7 .

\subsubsection{Neutrinos}

A neutrino candidate is defined in events where the missing transverse momentum $\not_{T}$ exceeds a value of $20 \mathrm{GeV}$. The missing momentum is derived from all identified particles and energy deposits in the event.

The four-vector of the neutrino can be calculated under the condition $\sum_{j}\left(E_{j}-P_{z, j}\right)$ equals twice the electron beam energy of $27.6 \mathrm{GeV}$. Furthermore it is assumed that the missing momentum is carried away by only one neutrino in the event. The Energy $E_{\nu}$ and polar angle $\theta_{\nu}$ of the neutrino is then given by

$$
E_{\nu}=\frac{P_{T}^{2}+\left(E-P_{z}\right)_{\nu}^{2}}{2\left(E-P_{z}\right)_{\nu}} \quad \text { and } \quad \cos \theta_{\nu}=\frac{P_{T}^{2}-\left(E-P_{z}\right)_{\nu}^{2}}{P_{T}^{2}+\left(E-P_{z}\right)_{\nu}^{2}} \quad \text {, }
$$

where $\left(E-P_{z}\right)_{\nu}=2 E_{0}-\sum_{i}\left(E_{i}-P_{z, i}\right)$ and $E_{0}$ denotes the electron beam energy [50].

Energy leakage through the beam pipe in the forward region of the H1 detector strongly degrades the neutrino identification. Therefore the phase space of the neutrino is restricted by the requirement $\sum_{i}\left(E_{i}-P_{z, i}\right)<48 \mathrm{GeV}$, efficiently suppressing falsely identified neutrino candidates from $\mathrm{NC}$ interactions.

In order to reduce misidentifications arising from the mismeasurement of other particles, the neutrino candidate is isolated against all identified high- $P_{T}$ objects by a minimum distance $D_{\nu}>1$ in the $\eta-\phi$ plane.

Furthermore events from NC interactions and lepton pair production may contribute as background, if one particle's energy is mismeasured. In those events the azimuthal angles of the charged lepton and the system $X$ built out of all other reconstructed particles 
are typically balanced, resulting in values $\Delta \phi_{l-X} \approx 180^{\circ}$. Therefore a neutrino is only reconstructed in events including an identified electron or muon if $\Delta \phi_{l-X}<170^{\circ}$.

The identification criteria are summarised in table 4.8. Further details on the neutrino selection can be found in [41].

\subsection{Event Classification}

The common phase space of electrons, photons, muons and jets is defined by the polar angle range $10^{\circ}<\theta<140^{\circ}$ and the transverse momentum requirement $P_{T}>20 \mathrm{GeV}$. The neutrino phase space is defined by missing transverse momentum above $20 \mathrm{GeV}$ and the condition $\sum_{i}\left(E_{i}-P_{z, i}\right)<48 \mathrm{GeV}$ on the longitudinal transverse momentum balance. These values are chosen to retain high selection and trigger efficiencies.

All high- $P_{T}$ particles including the neutrino defined by its reconstructed four-vector, are required to be isolated towards each other by a minimum distance $R=1$ in the $\eta-\phi$ plane to ensure the unambiguous identification of the final state topology.

Based on these object definitions the events are classified into exclusive event classes, according to the number and types of the selected objects.

Events with an isolated calorimetric object in the considered phase space which is not identified as photon, electron or jet are discarded from the analysis in order to minimise false classifications of events.

Figure 4.14 exemplarily presents the sum of transverse momenta distributions for the obtained $e-j, j-\nu$ and $j-j$ event classes ${ }^{6}$. These final state topologies originate from the dominating processes at HERA, i.e. neutral and charged current deep-inelastic scattering as well as the photoproduction of jets.

Shown are the measured number of data events and the individual distributions of the contributing SM processes. A good description of the data spectra by the prediction is observed, representing the good understanding of Standard Model physics up to the borders of phase space. The results for all event classes are discussed in section 6 .

\footnotetext{
${ }^{6}$ Potential detector corrections such as energy calibration and trigger efficiencies are already considered within the presented distributions (cf. chapter 5).
} 

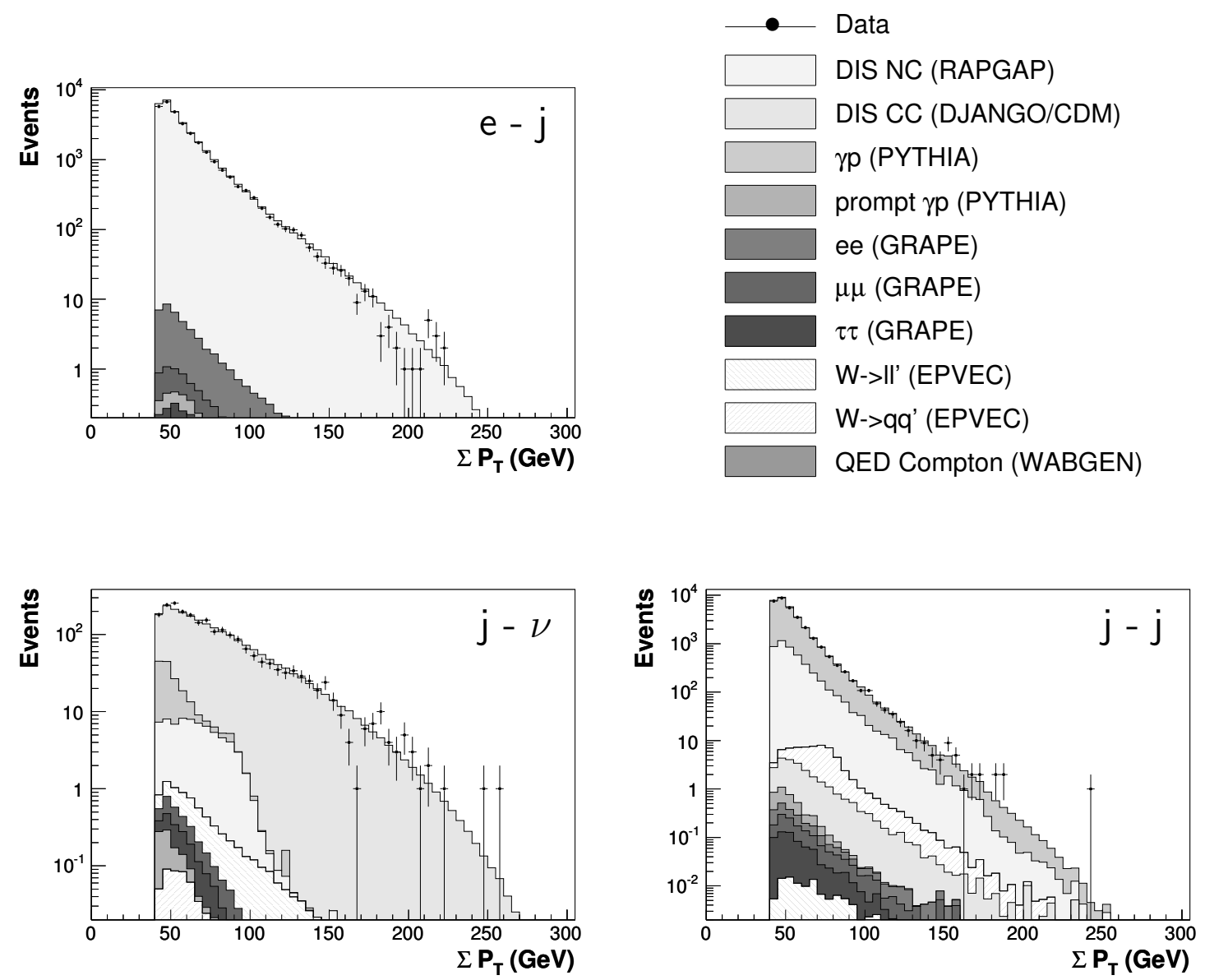

Fig. 4.14: The distributions of the sum of transverse momenta $\sum P_{T}$ for the $e-j$ (upper left), the $j-\nu$ (lower left) and the $j$ - $j$ (lower right) event classes. 


\section{Chapter 5}

\section{Experimental Performance}

A search for deviations between data and the SM expectation relies on a precise replication of the experimental measurement by the Monte Carlo simulation. In addition a thorough determination of the systematic uncertainties must be ensured. This chapter describes the studies pursued on the experimental performance and resulting detector corrections.

Both, the electromagnetic and hadronic energy scale uncertainty are investigated and the trigger efficiencies for the individual event configurations are considered. In addition some limitations of the measurement in dedicated event classes are discussed.

Subsequently the resolutions of the later on researched quantities $\sum P_{T}$ and $M_{\text {all }}$ are determined and purities as well as efficiencies for each event class are derived.

Finally the systematic uncertainties considered are discussed and the feasibility of a measurement of multi-jet final states is investigated.

\subsection{Energy Measurement and Calibration}

The following paragraphs describe the measurement of the electron and hadronic energy. The calibration methods are presented and the systematic errors on the electromagnetic and hadronic energy scale are determined.

\subsubsection{Electromagnetic Energy Scale}

The electron energy is determined by the energy deposit of the corresponding isolated and compact electromagnetic shower in the LAr calorimeter. A cone of radius $7.5^{\circ}$ originating from the event vertex around the centre-of-gravity of any calorimeter cluster defines the shower boundary. The total cluster energy is derived from all cells in the electromagnetic section and in the first layer of the hadronic section included in this cone.

The high- $Q^{2}$ electron energy calibration used in this analysis is based on the method described in [51] and is part of the electron identification software QESCAT (cf. section 4.3.1). Year dependent calibration factors are applied on both data and simulated Monte Carlo 


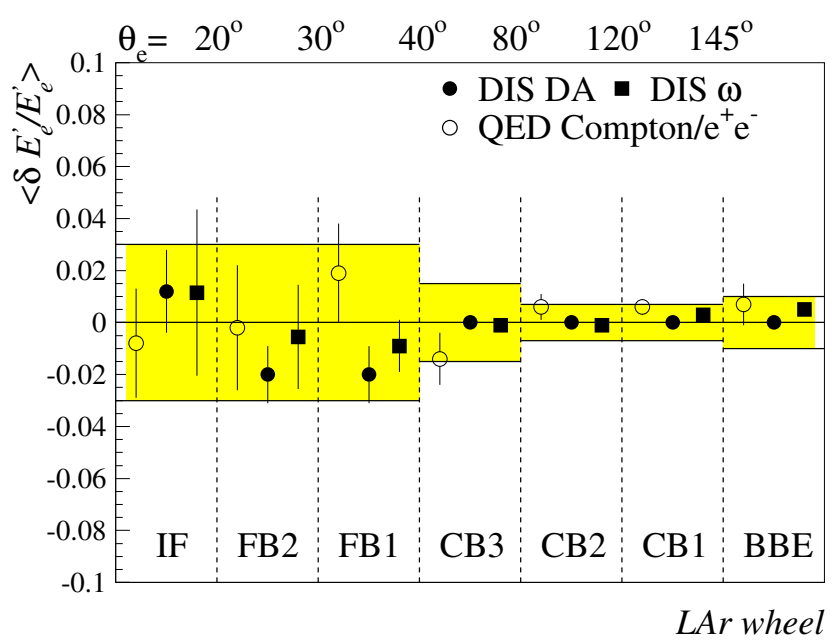

Fig. 5.1: Comparison of the electromagnetic energy scale as determined by different calibration methods. Shown is the mean fractional energy shift of the different methods from the absolute energy scale. The shaded error band shows the systematic uncertainty on the energy scale quoted on this analysis [51].

events as a function of the impact position of the electron to account for the $z$-dependent separation into wheels and the $\phi$-dependent octant structure of the LAr calorimeter.

Exploiting the over-constraint of the NC event kinematics the calibration factors are obtained by the comparison of the energies measured in the calorimeter with independently reconstructed values (cf. section 2.4). The most precise numbers are achieved using the double-angle reconstruction method as reference scale. The total uncertainty of the electromagnetic energy scale varies from $0.7 \%$ in the backward part of the calorimeter to $3 \%$ in the forward region, where the statistics is limited. The individual values depending on the region of the LAr calorimeter are presented in figure 5.1.

The quoted numbers on the electromagnetic energy scale uncertainty have been verified within the framework of this analysis. Further details can be found in [52].

\subsubsection{Hadronic Energy Scale}

The measurement of the hadronic final state is based on calorimeter as well as tracking information, exploiting the complementary precision of the momentum determination. Deposits in the LAr, SpaCal and Tail Catcher calorimeters are combined with the energies of low transverse momentum tracks to reconstruct the energy of the hadronic final state. Thereby the energy measurement in the LAr calorimeter contributes most.

Since the LAr calorimeter is non-compensating, software weighting methods are applied in order to improve the energy resolution. Therefore the recently developed "New Energy Weighting Scheme" [53] is used in the presented analysis. By this some deficits of the 
current method in the low energy regime are overcome.

Within a non-iterative procedure tabulated correction factors are applied on the measured energies to derive the reconstructed energies in each calorimeter cell. The weighting factors have been determined wheel-wise and separately for the electromagnetic and hadronic section of the LAr calorimeter using a detailed detector simulation of single $\pi$ events. In addition noise and dead material corrections are accounted for within this method.

In a second step calibration factors obtained from neutral current DIS data are applied, exploiting the over-constraint of the kinematics. Based on the precise electron energy calibration (cf. figure 5.1) these factors have been determined by adjusting the average values of the transverse momentum balance $P_{T}^{\text {had }} / P_{T}^{e}$ for both, data and Monte Carlo, to unity.

Since the central track detector provides a more precise measurement of low momentum particles than the LAr calorimeter, the energy resolution is further improved using a combination of the energies of low momentum tracks $\left(P_{T}<2 \mathrm{GeV}\right)$ originating from the event vertex and the energies deposited by other particles of the hadronic final state in the calorimeters. In order to avoid double counting, the amount of energy measured in the electromagnetic and hadronic section of the LAr calorimeter within a cylinder of radius $25 \mathrm{~cm}$ and $50 \mathrm{~cm}$, respectively, centred on the track direction is compared to the track energy. If the total energy in these cylinders exceeds the energy of the track, the calorimetric measurement is used solely. Otherwise those clusters with the smallest distance of closest approach to the track are discarded from the measurement until their energy contribution approximately matches the track energy.

In the framework of this analysis the calibration of the detector response to hadrons is finalised by the application of re-weighting factors on the transverse momenta of the reconstructed jets in order to further improve the energy resolution [41]. These final calibration factors are derived from the investigation of simulated neutral current DIS Monte Carlo events by adjusting the reconstructed jet transverse momenta $P_{T}^{j e t}$ to their generated values $P_{T}^{g e n}$. The obtained values of the transverse momentum balance $P_{T}^{\text {jet }} / P_{T}^{\text {gen }}$ were determined to match unity within $2 \%$ for the dependence on the transverse momentum as well as the polar angle of the reconstructed jet.

In order to confirm the derived hadronic energy scale uncertainty of $2 \%$ real neutral current DIS data are investigated. The electron transverse momentum is compared to the transverse momentum of both the reconstructed jet and the complete hadronic system in $e-j$ events, exploiting the precise calibration of the electron energy.

Based on the preselection presented in section 4.2 a clean NC event sample is selected including exactly one reconstructed electron with $P_{T}>15 \mathrm{GeV}$ as well as exactly one high transverse momentum jet identified in the considered polar angle range by the selection criteria described in section 4.3.1 and 4.3.4, respectively. A veto on any additional low transverse momentum jet with $P_{T}<5 \mathrm{GeV}$ is applied and at least $15 \mathrm{GeV}$ is demanded for the transverse momentum of the hadronic final state reconstructed with the Hadron 


\begin{tabular}{|c|}
\hline$P_{T}^{e}>15 \mathrm{GeV}$ and $10^{\circ}<\theta_{e}<140^{\circ}$ \\
\hline$N_{e}=1$ and $N_{\text {jet }}=1$ \\
\hline$P_{T}^{\text {had }}>15 \mathrm{GeV}$ \\
\hline $45 \mathrm{GeV}<\sum_{i}\left(E_{i}-P_{z, i}\right)<65 \mathrm{GeV}$ \\
\hline$y_{e}<0.9$ \\
\hline$P_{T}<15 \mathrm{GeV}$ \\
\hline
\end{tabular}

Tab. 5.1: The selection criteria for the neutral current DIS event sample used for the verification of the hadronic energy calibration.

method (cf. section 2.4).

In order to reject non-ep and photoproduction background the longitudinal momentum is demanded to be well balanced and the region of very high inelasticities $y_{e}$ is excluded. Missing transverse momentum is restricted to values below $15 \mathrm{GeV}$. The selection criteria are summarised in table 5.1.

Figure 5.2 presents the average values of the transverse momentum balance $P_{T}^{j e t} / P_{T}^{\text {da }}$ determined from both data and Monte Carlo as a function of the electron transverse momentum $P_{T}^{d a}$ reconstructed with the double-angle method and of the jet polar angle $\theta_{j e t}$, separately for the run periods 1994-1997 and 1998-2000. For both data and Monte Carlo the transverse momentum of the jet slightly undershoots the reconstructed electron value except for the region of small $P_{T}^{d a}$ where the impact of resolution causes a slow rise of the mean momentum balance. The uncertainty of the hadronic energy scale is derived from the level of agreement between the distributions of the transverse momentum balance determined from data and Monte Carlo. Besides the distributions of the average values the relative hadronic energy scale, i.e. the cross-ratio of $\left(P_{T, b a l}\right)^{M C}$ and $\left(P_{T, b a l}\right)^{\text {data }}$, is presented in figure 5.2. The diagrams corroborate that the differences between data and Monte Carlo amount to less than $2 \%$ over the whole transverse momentum and polar angle range for both the run period 1994-1997 and 1998-2000.

Since not all particles of the hadronic final state must be assigned to hard jets the transverse momentum of the electron has also been compared to the transverse momentum of the complete hadronic system reconstructed by the Hadron method. Figure 5.3 presents the obtained transverse momentum balance $P_{T}^{h a d} / P_{T}^{d a}$ as a function of $P_{T}^{d a}$ and $\theta_{j e t}$ as well as the cross-ratio of $\left(P_{T, \text { bal }}\right)^{M C}$ and $\left(P_{T, \text { bal }}\right)^{\text {data }}$. The values of the relative hadronic energy scale are again compatible with unity within an uncertainty of $2 \%$.

The uncertainty of the energy scale has also been investigated in multi-jet events mostly arising from photoproduction processes. While the NC DIS regime offers the possibility to adjust the hadronic energy to a precise scale determined by the electron calibration, the investigation of multi-jet events allows for an independent verification.

Using both a di-jet and three-jet photoproduction sample the transverse momentum balance of the two leading jets as well as the transverse momentum balance of the leading jet 

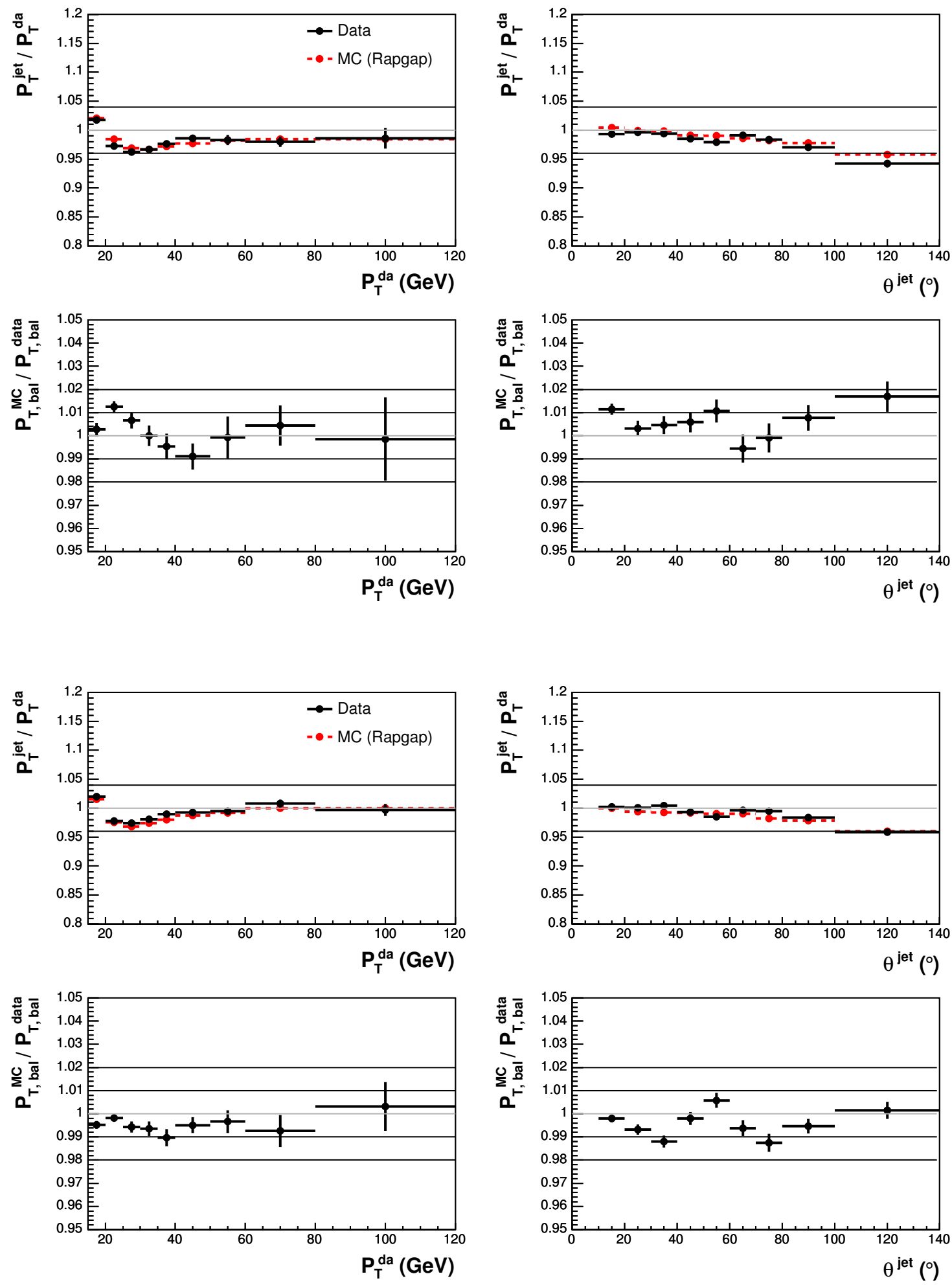

Fig. 5.2: The average values of the transverse momentum balance $P_{T}^{j e t} / P_{T}^{\text {da }}$ as a function of $P_{T}^{d a}$ and $\theta_{j e t}$ determined from data and Monte Carlo as well as the relative hadronic energy scale for the run period 1994-1997 (upper figures) and 1998-2000 (lower figures). 

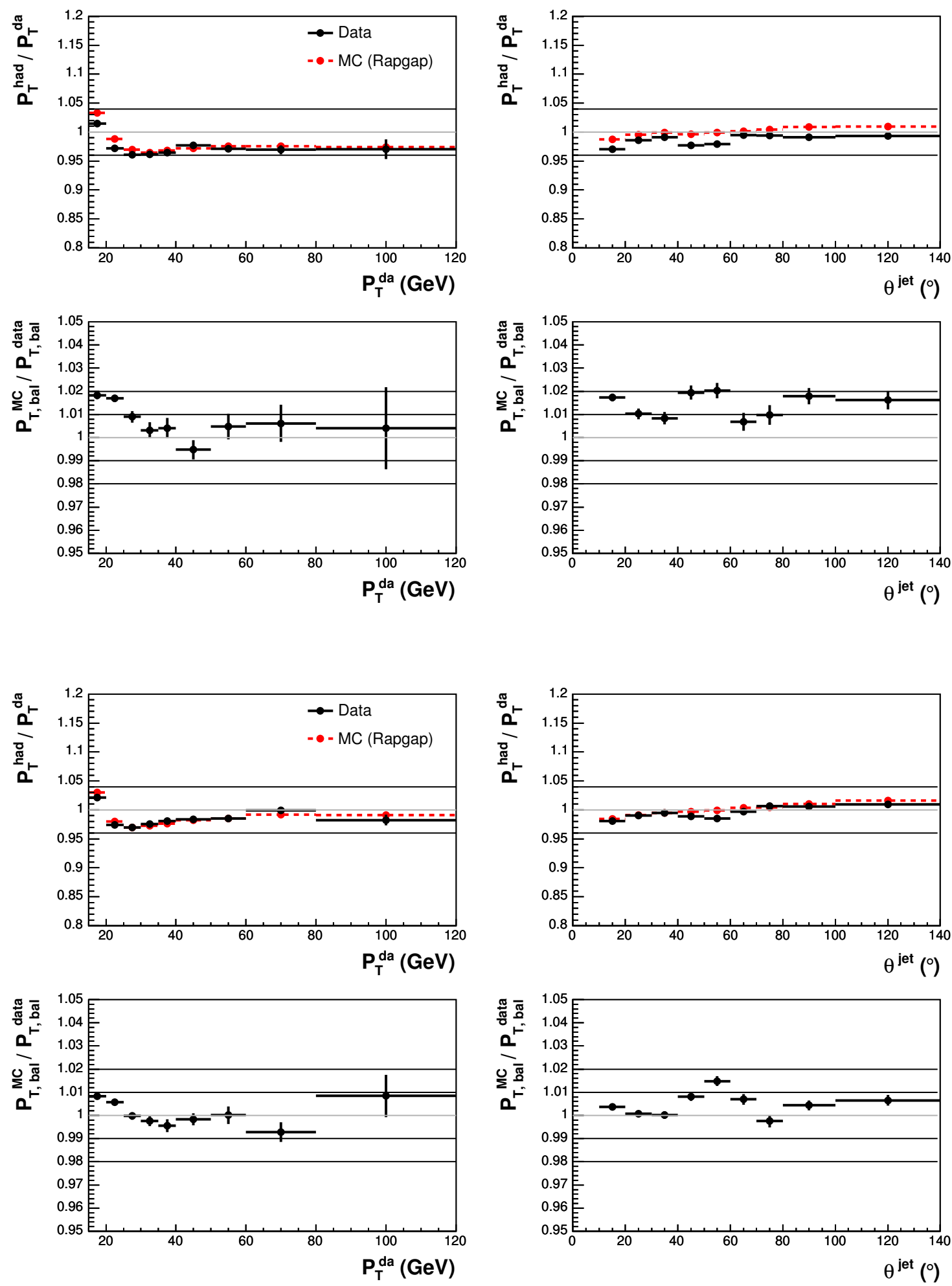

Fig. 5.3: The average values of the transverse momentum balance $P_{T}^{h a d} / P_{T}^{d a}$ as a function of $P_{T}^{d a}$ and $\theta_{j e t}$ determined from data and Monte Carlo as well as the relative hadronic energy scale for the run period 1994-1997 (upper figures) and 1998-2000 (lower figures). 
with the hadronic rest has been investigated within the framework of this analysis [41]. The studies pursued approve a systematic uncertainty of the hadronic energy scale in the LAr calorimeter of $2 \%$.

\subsection{Trigger Efficiencies}

The common phase space region of high transverse momentum ensures high trigger efficiencies for all event classes. Nevertheless remaining inefficiencies have been accounted for by correcting the Monte Carlo prediction according to the relevant trigger efficiencies determined in previous analyses on specific final states.

Since the trigger efficiencies determined in dedicated analyses are derived from specific final state topologies, the application to individual event classes considered in this analysis must not always be unambiguously defined. Anyhow, the classification of events in exclusive classes consisting of at least two high transverse momentum objects ensures high efficiencies, which can be accounted for by the disjunction of the relevant subtriggers and according efficiencies. In particular the trigger efficiencies for most event classes including more than two particles is assumed to amount close to $100 \%$.

The following paragraphs describe the individual subtriggers and their combined efficiencies considered for the measurement of the relevant event classes.

\section{Electrons and Photons}

Events including high electromagnetic energy deposits in the LAr calorimeter are efficiently triggered by the subtriggers S67 and S75, which are combined from LAr trigger tower signals $[16,54]$ with timing information from both the LAr calorimeter as well as the forward and central proportional chambers. Multiple calorimeter cells are summarised to so-called trigger towers, which are consecutively arranged in radial direction originating from the nominal event vertex. Caused by the detection of high energy deposited in one of the towers a trigger signal is delivered.

The combination of the subtriggers S67 and S75 is fully efficient for electromagnetic energies above $11 \mathrm{GeV}[10]$. Hence, the trigger efficiency is stated to be $100 \%$ in all event classes containing at least one electron or photon.

\section{Muons}

The muon subtriggers S19, S22, S34 and S56 are based on single muon signatures detected in different regions of the Central Muon Detector, which are combined with trigger signals from the Central Jet Chambers mainly specifying the track multiplicity and giving a rough estimate of the event vertex.

The efficiency of the combination of those subtriggers becomes relevant in events where no significant activity in the LAr calorimeter is measured. It has been determined to be typically $70 \%$ for di-muon events, well described by the detector simulation [42]. The 


\begin{tabular}{|l|c|}
\hline trigger & subtrigger \\
\hline \hline muon & $\mathrm{S} 19$ || S22 || S34 || S56 \\
jet & $\mathrm{S} 64$ || S67 || S75 || S77 \\
$P_{T}$ & $\mathrm{~S} 66$ || S67 || S71 || S75 || S77 \\
\hline
\end{tabular}

\begin{tabular}{|c|c|}
\hline event class & trigger condition \\
\hline \hline$\mu-\mu$ & muon trigger \\
$j-j$ & jet trigger \\
$\mu-j$ & muon or jet trigger \\
$j-\nu$ & $\not_{T}$ trigger \\
$\mu-j-\nu$ & muon or $P_{T}$ trigger \\
\hline
\end{tabular}

Tab. 5.2: The configuration of the muon, jet and $\not_{T}$ trigger (left side) and their assignment to event classes (right sight).

mentioned subtriggers are therfore required in this analysis for both data and Monte Carlo events divided into the $\mu-\mu$ event class.

\section{Jets}

Multi-jet events are triggered by the subtriggers S64, S67, S75 and S77. Although S67 and S75 are optimised for electrons they are also able to detect high-energetic jets. The subtriggers S64 and S77 are based on high transverse energy deposits and on the detection of missing transverse momentum in the LAr calorimeter, respectively, in coincidence with vertex and timing information.

The according efficiency of the combination of those subtriggers determined from a di-jet event sample amounts to $90 \%$ for $P_{T}>20 \mathrm{GeV}$ and nearly $100 \%$ for $P_{T}>25 \mathrm{GeV}[55,56]$. The quoted inefficiency is accounted for in the measurement of the $j$ - $j$ event class by requiring the mentioned subtriggers within the data measurement and re-weighting the Monte Carlo prediction depending on the transverse momentum and the pseudo-rapidity of the leading jet. Furthermore the resulting efficiency of the disjunction of the muon triggers with the jet subtriggers is considered for events populating the $\mu-j$ class.

\section{Neutrinos}

The subtriggers S66 and S77 are designed to trigger events with large missing transverse momentum, which is identified on trigger level by an imbalance in transverse momentum $P_{T}^{\text {calo }}$ determined from the vector sum of the LAr trigger towers. This information is combined with timing information delivered from both the LAr calorimeter as well as the forward and central proportional chambers. The efficiency is enhanced by additionally making use of the subtriggers S71 as well as S67 and S75, which are either mainly based on track activity in the Central Jet Chambers or primarily developed for the selection of events originating from neutral current processes.

The combined trigger efficiency of those subtriggers has been determined to be $\sim 90 \%$ for $P_{T}^{\text {calo }}>20 \mathrm{GeV}[10]$. It is considered in this analysis for events populating the $j-\nu$ class by the requirement of the mentioned subtriggers for the data measurement and the according adjustment of the Monte Carlo prediction depending on the transverse momentum $P_{T}^{\text {had }}$ and the inclusive angle $\gamma$ of the hadronic final state. The resulting efficiency of 
the disjunction of the $P_{T}$ subtriggers with the muon subtriggers is accounted for in the measurement of the $\mu-j-\nu$ event class.

The configuration of the muon, jet and $P_{T}$ trigger and their consideration in this analysis is summarised in table 5.2.

\subsection{Limitations of the Measurement}

In the presented analysis all final states with at least two high- $P_{T}$ particles arising from $e p$ interactions have been studied for the first time at HERA. The identification criteria have been chosen to ensure an unambiguous identification of all considered particles.

Nevertheless, two event classes are affected by limitations of the measurement. Though the cause for the restrictions originates from different aspects, the event classes concerned are those including missing transverse momentum and a high- $P_{T}$ lepton.

The following paragraphs describe those limitations and their impact on the analysis.

\subsubsection{The $e-\nu$ Event Class}

In the $e-\nu$ event class 9 data events are measured while 19.9 events are predicted by the Standard Model. Though this topology is characteristic for events arising from $W$ production, the dominant contribution to the SM expectation comes from neutral current DIS events, where fluctuations in the hadronic energy measurement or limited detector acceptance can produce missing transverse momentum.

The large size of the discrepancy observed is caused by limitations in the simulation of low-energetic hadronic energy deposits in the forward region of the $\mathrm{H} 1$ detector. In order to quantify the disagreement between the measurement and simulation an inclusive low$P_{T}$ electron event sample is studied. Based on the preselection described in section 4.4 events including electrons with $P_{T}>10 \mathrm{GeV}$ are collected. Furthermore the longitudinal momentum balance $\sum_{i}\left(E_{i}-P_{z, i}\right)$ is restricted to values below $48 \mathrm{GeV}$ in order to match the phase space considered for the neutrino identification.

Figure 5.4 presents the missing transverse momentum distributions derived from the selected event sample. The diagram on the left side shows the $P_{T}$ distribution obtained from the complete inclusive sample. It illustrates an overshoot of the NC DIS prediction for high values of $\mathbb{P}_{T}$. Only in the region of very high missing transverse momenta, where the rate of events originating from $W$ production measures up to the contribution of NC DIS events, the number of data events roughly agrees with the SM expectation.

In order to verify the discrepancy observed and to determine a re-weighting factor those events including signal electrons, i.e. electrons with $P_{T}>20 \mathrm{GeV}$, are excluded from the inclusive event sample. The obtained $P_{T}$ distribution is shown in the diagram on the right side of figure 5.4. Even though the data statistics is limited it corroborates the overshoot of the NC DIS prediction at high values of $P_{T}$.

Resulting from the studies of the presented distributions the prediction of NC DIS events 

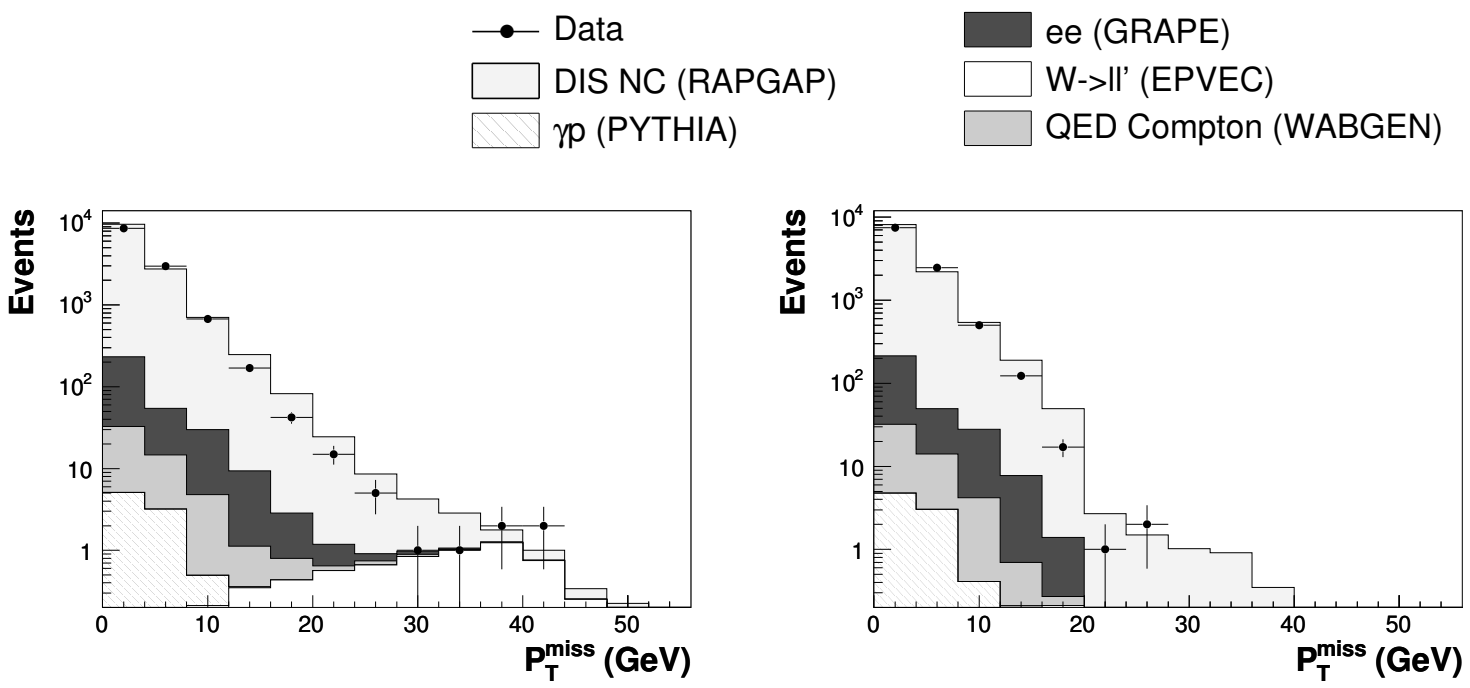

Fig. 5.4: The $P_{T}$ distributions for events with $\sum_{i}\left(E_{i}-P_{z, i}\right)<48 \mathrm{GeV}$ obtained from an low- $P_{T}$ electron sample. While the left figure is based on the inclusive sample, only events including electrons with $P_{T}<20 \mathrm{GeV}$ are considered for the distribution presented on the right side.
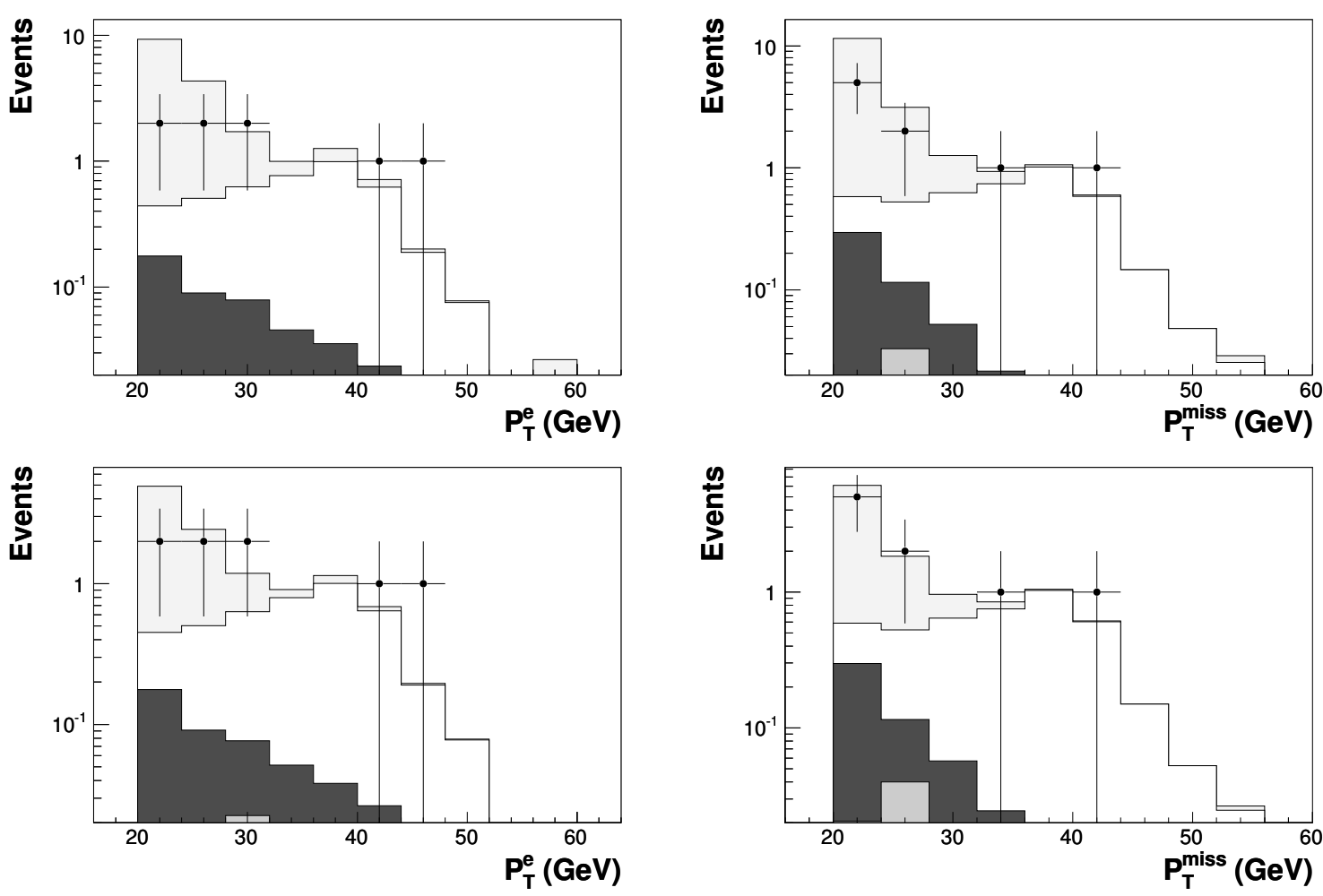

Fig. 5.5: The distributions of $P_{T}^{e}$ and $\not_{T}$ for the $e-\nu$ event class before (upper figures) and after (lower figures) re-weighting the NC DIS prediction. 
containing missing transverse momentum above $20 \mathrm{GeV}$ and a high- $P_{T}$ electron is lowered to its half. To account for the uncertainty of this procedure a systematic error of $50 \%$ is considered.

Figure 5.5 presents the $P_{T}$ distribution of the electron and the $P_{T}$ distribution of the neutrino for the $e-\nu$ class, before and after the application of the re-weighting procedure. After re-weighting the NC DIS contribution the total number of predicted events amounts to $12.9 \pm 4.5$, still dominated from background.

\subsubsection{The $\mu-\nu$ Event Class}

A large discrepancy between the data and the expectation is observed in the $\mu-\nu$ event class. In total 93 data events are measured while only 5.6 events are obtained from the used Monte Carlo event samples (cf. table 3.1). Within this expectation about 4 events arise from photoproduction. In addition 0.97 events originating from di-muon production processes contribute and 0.51 events from $W$ production are predicted. The obtained distributions of the transverse momenta as well as azimuth and polar angles of the muon and neutrino reconstructed in the $\mu-\nu$ event class are presented in figure 5.6.

The large size of the deviation between the data and the expectation reveals a fundamental problem in the measurement of this event class, whose reason might in general be explained by two possibilities: Either the data contain a significant fraction of non-ep background events or not all possible physics processes are contained or well simulated in the prediction.

The investigation of the experimental signatures of the observed data events suggest that the $\mu-\nu$ event class is dominated by background arising from low- $P_{T}$ photoproduction processes. Within a small fraction among the large number of low- $P_{T}$ photoproduction events the track of a low- $P_{T}$ muon contained in the hadronic final state might wrongly be reconstructed, producing high missing transverse momentum. Thus these events are satisfying the conditions of the $\mu-\nu$ event class.

This assumption is corroborated by figure 5.7, which shows the distribution of the transverse momentum of the leading jet for those events of the $\mu-\nu$ event class, where a jet has been reconstructed by the jet algorithm ${ }^{1}$. The distribution reveals that most of the data events have either no jet or a jet with a transverse momentum below $10 \mathrm{GeV}$ as it is expected for low- $P_{T}$ photoproduction events.

In this analysis the SM prediction for low- $P_{T}$ photoproduction is considered down to $\hat{P}_{T}$ values of $5 \mathrm{GeV}$, already with a low luminosity half as much as the luminosity of the data for $\hat{P}_{T}$ values below $10 \mathrm{GeV}$ (cf. chapter 3 ). Since the computing technology is even today not yet capable to cope with the large amount of Monte Carlo events which would be needed for the coverage of the inclusive low- $P_{T}$ photoproduction regime, the $\hat{P}_{T}$ require-

\footnotetext{
${ }^{1}$ A minimal transverse momentum of $3 \mathrm{GeV}$ is required by the jet algorithm. It might further be noted that also high- $P_{T}$ jets not reconstructed within the considered polar angle range of $10^{\circ}<\theta<140^{\circ}$ enter the presented distribution.
} 

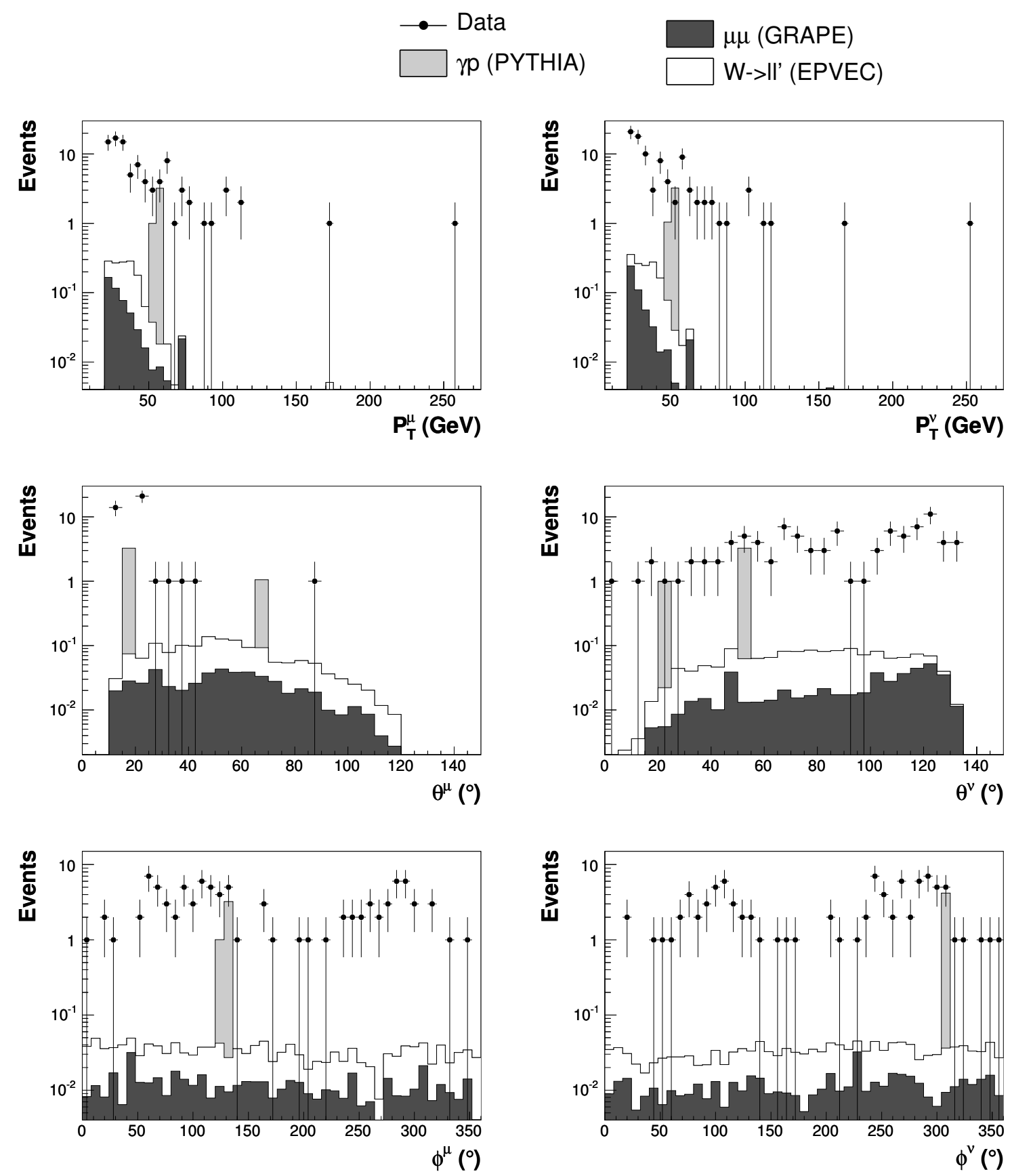

Fig. 5.6: The distributions of the transverse momenta $P_{T}$, the azimuth angles $\phi$ and the polar angles $\theta$ for the muon (left side) and neutrino (right side), reconstructed in the $\mu-\nu$ event class. 


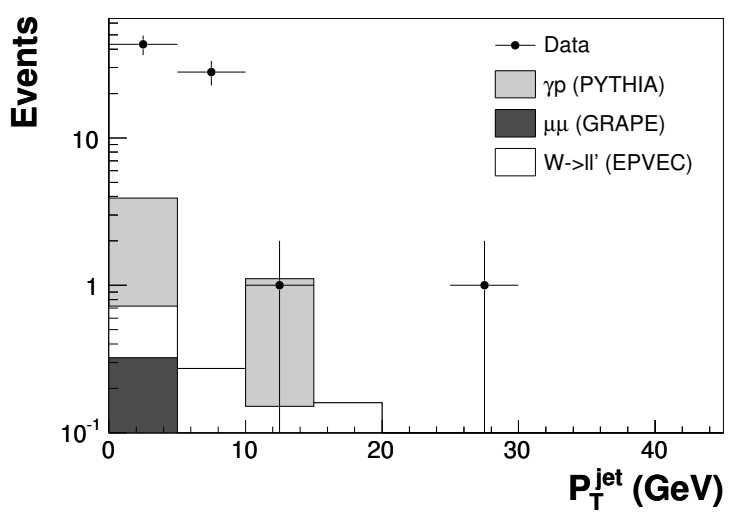

Fig. 5.7: The distribution of the transverse momentum $P_{T}$ of the leading jet for the $\mu-\nu$ event class.

ment could not further been lowered in the event generation ${ }^{2}$.

Comparing the number of 4 predicted events with the value of the photoproduction cross section for $\hat{P}_{T}>5 \mathrm{GeV}$ in the order of $2 \cdot 10^{5} \mathrm{pb}$, only about 1 out of $6 \cdot 10^{6}$ events are expected to comprise a muon with a wrongly reconstructed transverse momentum greater than $20 \mathrm{GeV}$. Assuming a photoproduction cross section between $10^{6} \mathrm{pb}$ and $10^{7} \mathrm{pb}$ for $\hat{P}_{T} \gtrsim 2 \mathrm{GeV}$, which is approximately the value needed for the muon to be observable within the H1 detector, between 20 and 200 photoproduction events would be expected to contribute to the $\mu-\nu$ event class. These values are roughly in agreement with the number of 93 observed data events.

Consequently, the deviation between the data and expectation might be explained by a not considered background contribution from low- $P_{T}$ photoproduction processes, where a low- $P_{T}$ muon track is wrongly reconstructed. Anyhow, besides the fact that it is technically not feasible to give a better estimation of the photoproduction background in the $\mu-\nu$ event class within this analysis, the uncertainty remains if the detector simulation is able to reproduce the data measurement up to this high precision of $\mathcal{O}\left(10^{-6}\right)$.

Apart from the contribution of imprecisely known low- $P_{T}$ processes, another source of background might contaminate the measurement of this event topology.

Investigating the azimuth angle distribution of the muons selected in the data, a conspicuous accumulation of events is observed for values around $90^{\circ}$ and $270^{\circ}$. This signature is typically produced by muons from cosmic radiation, vertically crossing the detector. On the other hand the distribution of the polar angle $\theta$ shows, that most of the muons are located in forward region $\theta<25^{\circ}$, stronger arguing for the assumption of wrongly reconstructed muon tracks in low- $P_{T}$ photoproduction events.

Anyhow, it can not be fully excluded that a fraction of the data are photoproduction events with an overlaid cosmic muon, which has neither been identified by the topological

\footnotetext{
${ }^{2}$ Assuming a cross section in the order of $10^{7} \mathrm{pb}$ about $10^{9}$ events must be simulated just to obtain a luminosity of the Monte Carlo sample in the order of the data luminosity.
} 
background finders (cf. sections 4.2 and 4.3.3) nor by the intensive visual scanning of events performed in particular for this event class.

For both discussed reasons it is decided to discard the $\mu-\nu$ event class from further analyses. Nevertheless, it might be noted that both kinds of background contributions are negligible for the according event class including an additional high transverse momentum jet, represented by the $\mu-j-\nu$ event class.

Assuming, that in the same way as for the $\mu-\nu$ event class photoproduction events with mismeasured muons or overlaid muons from cosmic radiation contribute to the $\mu-j-\nu$ event class, these events must now originate from high- $P_{T}$ photoproduction processes. Taking into account the derived rate in the order of $10^{-6}$, with that this background topology is expected to appear, the contribution to the $\mu-j-\nu$ event class can by estimated from the total number of $\sim 3 \cdot 10^{4}$ events measured in the $j$ - $j$ event class. This approximation results in a small value of 0.03 events. Moreover, due to the physics process of photoproduction this scenario would typically manifest itself in the existence of a second jet with a transverse momentum just under $20 \mathrm{GeV}$. This has not been observed in any of the data events in the $\mu-j-\nu$ event class.

Since a contribution of this kind of background in photoproduction is excluded for the $\mu-j-\nu$ event class, one might finally assign the same consideration on events including already real missing transverse momentum, as it is the case for events arising from charged current processes. But since the rate observed in the $j-\nu$ events class is more than a factor of 10 smaller than that for di-jet events, also charged current events including a mismeasured muon can be excluded to contribute to the $\mu-j-\nu$ event class.

\subsection{Resolutions}

In order to search for new physics signals hidden in the data a dedicated algorithm is presented in chapter 7 , which locates the region of largest deviation from the SM expectation in the sum of transverse momenta and invariant mass distributions. To account for statistical fluctuations the minimal size of the considered phase space is restricted to a size in the order of twice the resolution of the researched observable.

The resolution of $\sum P_{T}$ and $M_{\text {all }}$ for all event classes with a sizeable SM expectation are determined from simulation by comparing the reconstructed and generated values. In addition to the classification on reconstruction level all events are therefore subdivided into exclusive classes based on generator information. In the same way as for the reconstructed objects only particles identified in the common phase space region specified in section 4.4 are considered for the classification on generator level.

Furthermore a matching criterion is introduced, according to which a reconstructed particle has to be uniquely assigned to a generated one and vice versa. Taking into account the different types of the particles a minimum distance of $R<1.5$ in the $\eta-\phi$ plane is required in order to match the generated and reconstructed particle. 

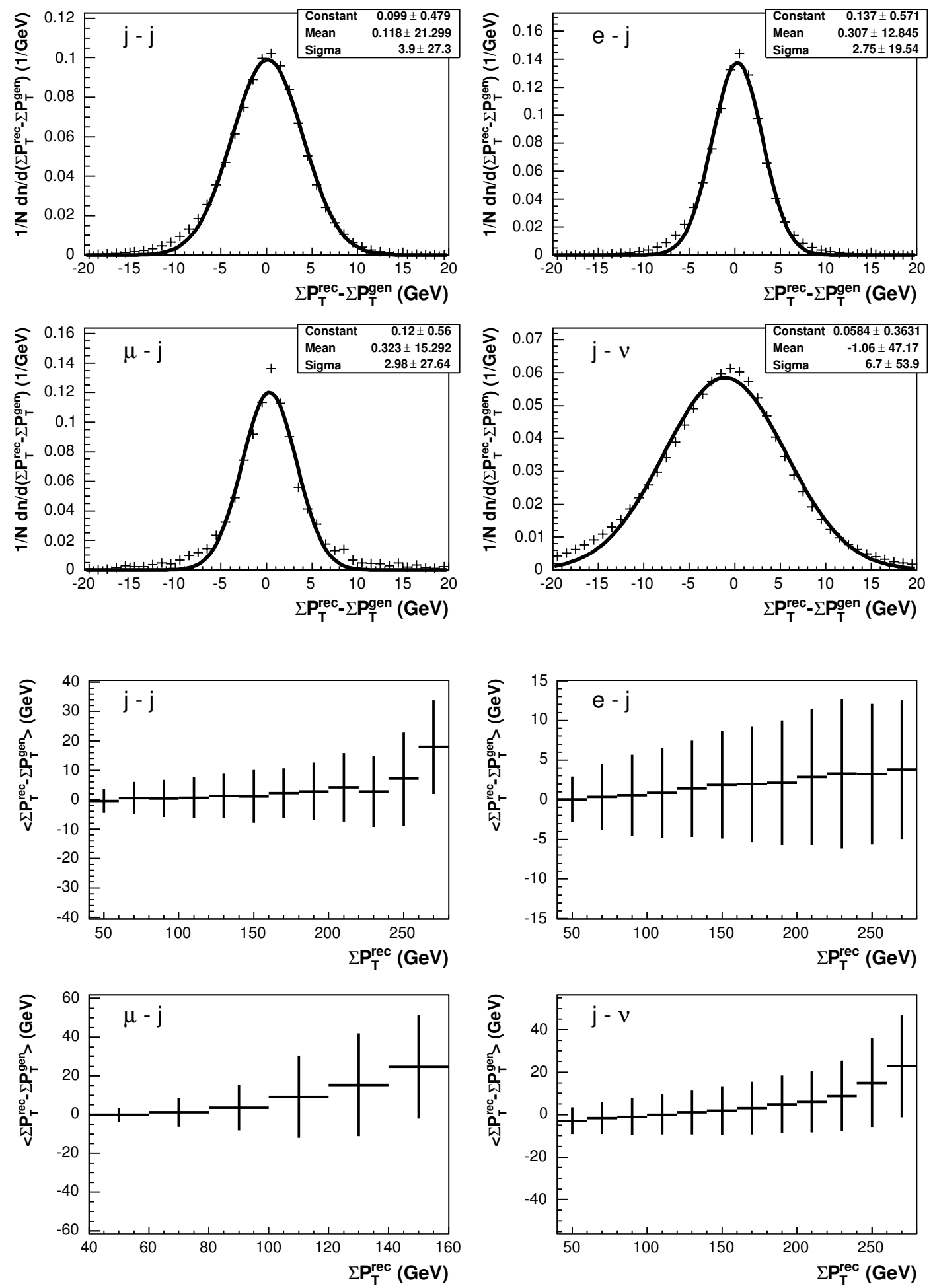

Fig. 5.8: The resolution of $\sum P_{T}$ (upper figures) and its dependence on the reconstructed values of $\sum P_{T}$ (lower figures) for the $j-j, e-j, \mu-j$ and $j-\nu$ event classes. 
For the events with matched particles and subdivided into the same event class on reconstruction and generator level the resolution is determined by fitting a Gaussian function to the distributions of the difference between the generated and reconstructed $\sum P_{T}$ and $M_{\text {all }}$ values, respectively. The obtained resolutions are depicted in the upper part of figure 5.8 exemplarily for four different event classes. The distributions for all event classes containing data events are presented in appendix A.

Table A.1 summarises the determined resolutions $\delta$ of $\sum P_{T}$ and $M_{\text {all }}$. Values in the order of $2 \mathrm{GeV}$ are found in event classes with two electromagnetic particles only $(e-e, e-\gamma)$, while a resolution of $14 \mathrm{GeV}$ is derived for the invariant mass in the $\mu-j-\nu$ class.

In addition to the absolute values of the resolution their dependence on the reconstructed sum of transverse momenta and invariant mass has been investigated, exemplarily presented in the lower part of figure 5.8. For increasing values of $\sum P_{T}$ and $M_{\text {all }}$ worse resolutions are found compared to the absolute values, which are dominated by the statistically high populated regions of low $\sum P_{T}$ and $M_{\text {all }}$ values.

Though a slight variation of the resolutions as a function of $\sum P_{T}$ and $M_{\text {all }}$ has been noticed, the consideration of fixed values used by the search algorithm has been found to be a sufficient approximation. In order to derive a quantitative estimate of the resolutions over the whole range of $\sum P_{T}$ and $M_{\text {all }}$ both the arithmetic mean $\langle\delta\rangle$ and event weighted mean $\langle\delta\rangle_{w}$ have been calculated, likewise presented in table A.1. Based on these average values the minimal region size considered by the search algorithm is determined for all event classes.

\subsection{Purities and Efficiencies}

In order to study the rejection power of the particle identification criteria (cf. section 4.3) and to evaluate the unambiguousness of the event classification purities and efficiencies are derived for all event classes. Furthermore the determination of selection efficiencies provides the opportunity to quantify the discovery potential of the analysis and can be used to set exclusion limits on new physics signals.

Though presenting an interesting approach the latter will not be covered in the framework of the present work. Instead of that the sensitivity to new physics signals is directly been probed by mixing the prediction of specific models to the SM expectation and the research of the obtained distributions using the developed algorithm (cf. section 7.3).

Purities and efficiencies for all event classes with a sizeable SM expectation are determined from simulation by comparing the number of events subdivided into the according event classes on generator and reconstruction level. Compared to the method described in section 5.4 no matching criterion based on the spatial distance is applied on the identification of the individual reconstructed and generated particles.

The purity $\mathcal{P}$ for each event class is defined as the ratio of SM events reconstructed in the specific class in which they have been generated to the total number of reconstructed events in that class. Likewise the efficiency $\mathcal{E}$ is given by the number of events both gener- 

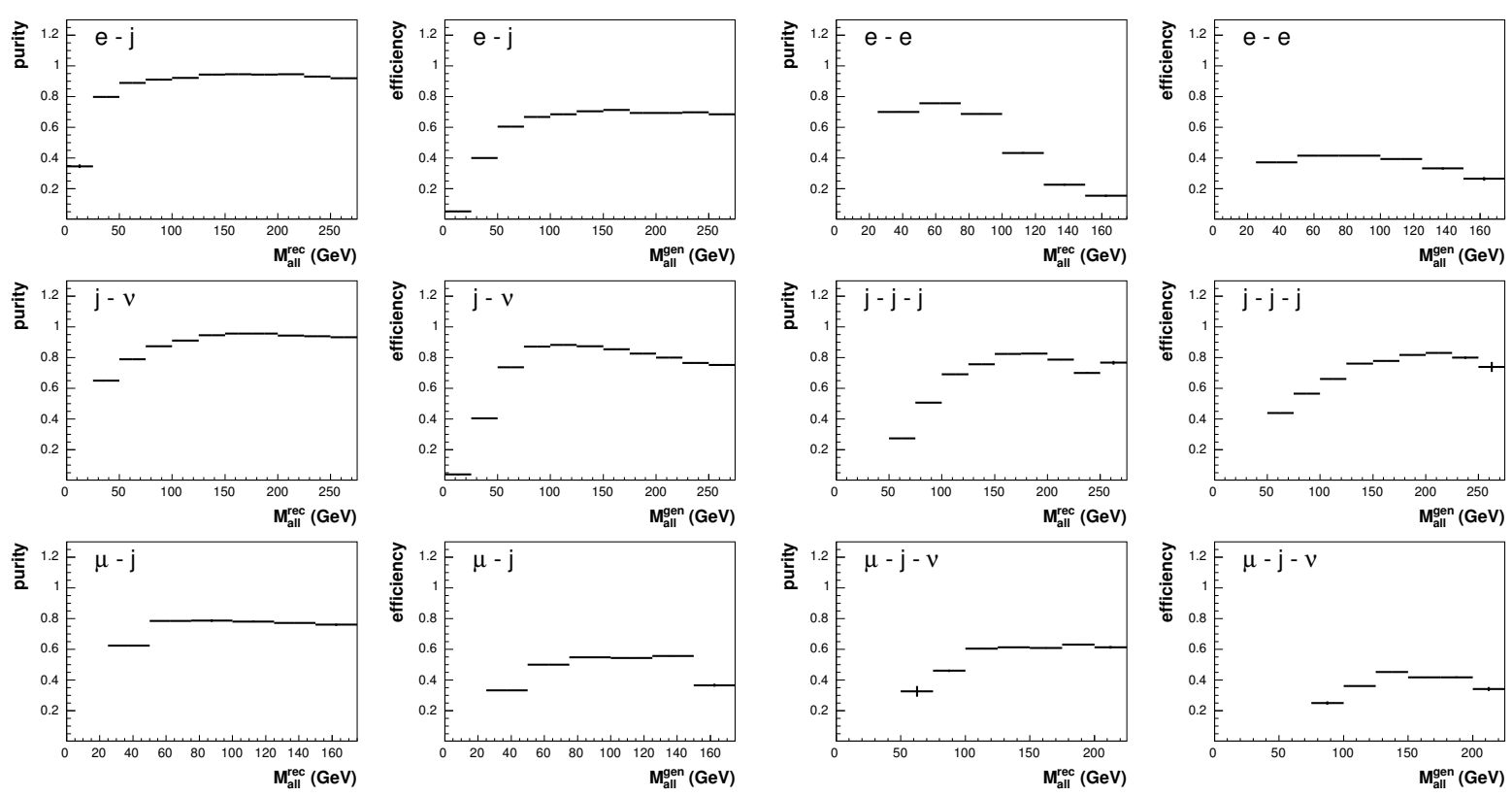

Fig. 5.9: Purities and efficiencies as a function of the invariant mass $M_{\text {all }}$ for various event classes.

ated and reconstructed in a common event class divided by the total number of generated events in that class:

$$
\mathcal{P}=\frac{N_{\text {rec } \wedge \text { gen }}(\text { class })}{N_{\text {rec }}(\text { class })} \quad \mathcal{E}=\frac{N_{\text {rec } \wedge \text { gen }}(\text { class })}{N_{\text {gen }}(\text { class })} .
$$

The purity quantifies therefore the contribution of genuine events ${ }^{3}$ to an event class while the efficiency measures the finding potential. Both quantities are derived as a function of the sum of transverse momenta and the invariant mass, the purity depending on the reconstructed and the efficiency depending on the generated values.

In order to correct for the impact of geometrical acceptance both generated and reconstructed particles have to be identified in the common phase space defined in section 4.4, accepting limitations arising from migration effects at the cut boundaries. In addition to the actual misidentification of particles the loss of purity and efficiency resulting from the exclusion of inefficient detector regions as well as from the not fully hermetical design of the Central Muon Detector remains unaffectedly considered.

As already discussed in section 4.3 .4 a high purity of $90 \%$ for invariant masses above $100 \mathrm{GeV}$ is obtained for di-jet events still ensuring a high selection efficiency of $95 \%$.

Further distributions of purities and efficiencies as a function of the invariant mass $M_{\text {all }}$ for various event classes are presented in figure 5.9. Even higher purities of up to $95 \%$ are found in the $e-j$ and $j-\nu$ event class. Strongly dominated by the track association efficiency a selection efficiency of $70 \%$ is obtained in the $e-j$ event class, while the efficiency

\footnotetext{
${ }^{3}$ Assuming the absence of new physics signals.
} 
in the $j-\nu$ class amounts to $90 \%$, slightly decreasing for high masses.

Resulting from limitations of the geometrical acceptance an efficiency of $55 \%$ is found in the $\mu-j$ event class, where the contribution of genuine events amounts to $80 \%$.

As expected a selection efficiency of roughly half the value obtained from $e-j$ events is found for the $e$-e event class. The reinforced loss of purity at high invariant mass indicates a remaining background contribution arising from QED Compton and NC DIS events due to electron misidentification in the forward polar angle region.

In the same way as for di-jet events a high purity and efficiency is found in the $j-j-j$ event class. Both distributions reach values of up to $85 \%$. Furthermore the gradual reduction in the region of low invariant masses illustrates the impact of migration, increasing with the number of particles contained in the event classes.

The selection efficiency for $\mu-j-\nu$ events is found to be $\sim 40 \%$ for invariant masses above $100 \mathrm{GeV}$, compatible with values derived in [37]. The contribution of genuine events to this class amounts to $65 \%$.

The purities and efficiencies as a function of both the sum of transverse momenta and the invariant mass for all event classes containing data events are presented in appendix B. Most purities are found to be above $60 \%$ reaching $100 \%$ in dedicated event classes. The highest efficiencies are found in the already presented $j-j, j-\nu$ and $j-j-j$ event class. In case of observing deviations between the data and SM expectation in regions of low purity this has to be considered in the interpretation of the results.

\subsection{Systematic Uncertainties}

This section describes the sources of systematic uncertainties considered. All experimental uncertainties are determined from data based on studies either performed in the framework of this analysis or in dedicated measurements on specific final states. The systematic uncertainties arising from the measurement of the particles are summarised in table 5.3.

\section{Uncertainty in the Electron and Photon Reconstruction}

As illustrated in section 5.1.1 the high- $Q^{2}$ electron calibration, primarily developed for the measurement of the inclusive neutral current cross section [10], is used in this analysis. The uncertainty of the electromagnetic energy scale varies from $0.7 \%$ in the backward part to $3 \%$ in the forward region, depending on the calorimeter wheel in which the impact point of the electromagnetic particle is reconstructed (cf. figure 5.1).

The systematic uncertainty in the polar angle measurement of electromagnetic clusters has been determined to range from $1 \mathrm{mrad}$ for $\theta>135^{\circ}$ to $3 \mathrm{mrad}$ for $\theta<120^{\circ}$ [10].

The identification efficiencies for electrons and photons have been studied in section 4.3.1 and 4.3.2. Uncertainties arise from discrepancies in the simulation of the electron track association and the hit efficiency of the CIP. The absolute error of the track efficiency varies depending on the polar angle of the electromagnetic cluster from $2 \%$ in the central 


\begin{tabular}{|l|c|c|c|}
\hline object & energy scale & $\theta(\mathrm{mrad})$ & identification efficiency \\
\hline \hline electron & $0.7-3 \%$ & $1-3$ & $2-7 \% \oplus 0.5 \%$ \\
photon & $0.7-3 \%$ & $1-3$ & $2-7 \% \oplus 0.5 \%$ \\
muon & $5 \%$ & 3 & $5 \%$ \\
jet & $2 \%$ & $5-10$ & - \\
\hline
\end{tabular}

Tab. 5.3: The systematic uncertainties attributed to the particle measurements.

region $\left(\theta>37^{\circ}\right)$ to $7 \%$ in the forward part. The systematic uncertainty of the CIP hit efficiency amounts to $0.5 \%$ over the whole polar angle range.

\section{Uncertainty in the Muon Reconstruction}

The systematic uncertainty in the transverse momentum measurement for muons amounts to $5 \%$, determined from the $P_{T}$ balance using a di-muon data sample [57]. The absolute error on the polar angle measurement is $3 \mathrm{mrad}$ and an uncertainty of $5 \%$ is considered for the muon identification efficiency [42, 57].

\section{Uncertainty in the Hadronic Final State Measurement}

The hadronic energy measurement has been probed in detail within the framework of the this analysis (cf. section 5.1.2). An uncertainty of $2 \%$ is determined for the hadronic energy scale of the LAr calorimeter, corroborated from studies of neutral current DIS events as well as from the investigation of multi-jet events in the kinematic regime of photoproduction. The systematic uncertainty on the jet polar angle measurement amounts to $5 \mathrm{mrad}$ for $\theta<30^{\circ}$ and to $10 \mathrm{mrad}$ otherwise.

\section{Uncertainty in the Trigger Efficiency}

The consideration of trigger efficiencies has been presented in section 5.2. Uncertainties are taken into account according to the particle with the highest trigger efficiency. An error of $3 \%$ is applied for events triggered by a jet while $5 \%$ are considered for events triggered by muons. Otherwise the uncertainty in the trigger efficiency is negligible.

\section{Uncertainty in the Luminosity Determination}

The uncertainty in the luminosity determination is $1.5 \%$ introducing a corresponding overall normalisation error.

\section{Statistical Uncertainties}

Statistical uncertainties arise from the finite number of Monte Carlo events. Since all SM processes are generated with an integrated luminosity significantly higher than that of the data sample, the impact of statistical uncertainties is small. 


\begin{tabular}{|lll|c|}
\hline process $(\mathrm{es})$ & & & uncertainty \\
\hline \hline$e p \rightarrow j j X$ & and & $e p \rightarrow j \gamma X$ & $15 \%$ \\
$e p \rightarrow j \nu X$ & and & $e p \rightarrow j e X$ & $10 \%$ \\
$e p \rightarrow j j \nu X$ & and & $e p \rightarrow j j e X$ & $15 \%$ \\
$e p \rightarrow \mu \mu X$ & and & $e p \rightarrow e e X$ & $3 \%$ \\
$e p \rightarrow W X$ & and & $e p \rightarrow W j X$ & $15 \%$ \\
$e p \rightarrow e \gamma X$ & and & $e p \rightarrow e \gamma j$ & $10 \%$ \\
$e p \rightarrow e \gamma p$ & & & $5 \%$ \\
\hline
\end{tabular}

Tab. 5.4: Theoretical uncertainties attributed to different SM processes.

\section{Theoretical Uncertainties}

Depending on the dominant production process different theoretical uncertainties are considered, listed in table 5.4 .

An error of $15 \%$ is attributed to the photoproduction processes of jets and photons $(e p \rightarrow j j X, e p \rightarrow j \gamma X)$ including uncertainties in the parton distribution functions and those arising from missing higher order corrections [19, 58].

For the same reasons an uncertainty of $10 \%$ is considered for the neutral and charged current deep-inelastic scattering processes on Born level $(e p \rightarrow j e X$, ep $\rightarrow j \nu X)$ while an error of $15 \%$ is applied on the QCD Compton and Boson Gluon Fusion processes $e p \rightarrow j j e X$ and $e p \rightarrow j j \nu X$.

Mainly resulting from uncertainties in the structure functions an error of $3 \%$ is attributed to the electroweak lepton pair production processes $e p \rightarrow \mu \mu X$ and $e p \rightarrow e e X$ [38, 42].

The uncertainty in the QED Compton cross section has been estimated to amount to $5 \%$ for elastic $(e p \rightarrow e \gamma p)$ and $10 \%$ for the inelastic production $(e p \rightarrow e \gamma X, e p \rightarrow e \gamma j)$.

The error of $15 \%$ on the $W$ production cross section is mainly arising from missing higher order corrections [37].

An additional theoretical error of $20 \%$ is applied for each jet dominantly produced by parton shower processes (e.g. $j-j-j$ event class) to account for differences between the parton shower and dipole model approach as well as higher order QCD calculations.

A model uncertainty of $50 \%$ is added to neutral current DIS events including an identified high- $P_{T}$ electron together with large missing transverse momentum above $20 \mathrm{GeV}$ (cf. section 5.3.1).

All systematic errors are added in quadrature. Since the algorithm searching for deviations between data and the SM prediction (cf. chapter 7) relies on smooth distributions, the resulting uncertainties considered as a function of the researched quantities are fitted. Figure 5.10 exemplarily presents the obtained systematic uncertainties for the $e-j$ and $j-j$ event class as a function of $\sum P_{T}$ and $M_{\text {all }}$, respectively.

The total error for $e-j$ events ranges from $10 \%$ at low up to $50 \%$ at high $\sum P_{T}$ values. The uncertainty amounts to $25 \%$ for values around $220 \mathrm{GeV}$, the region populated by the 

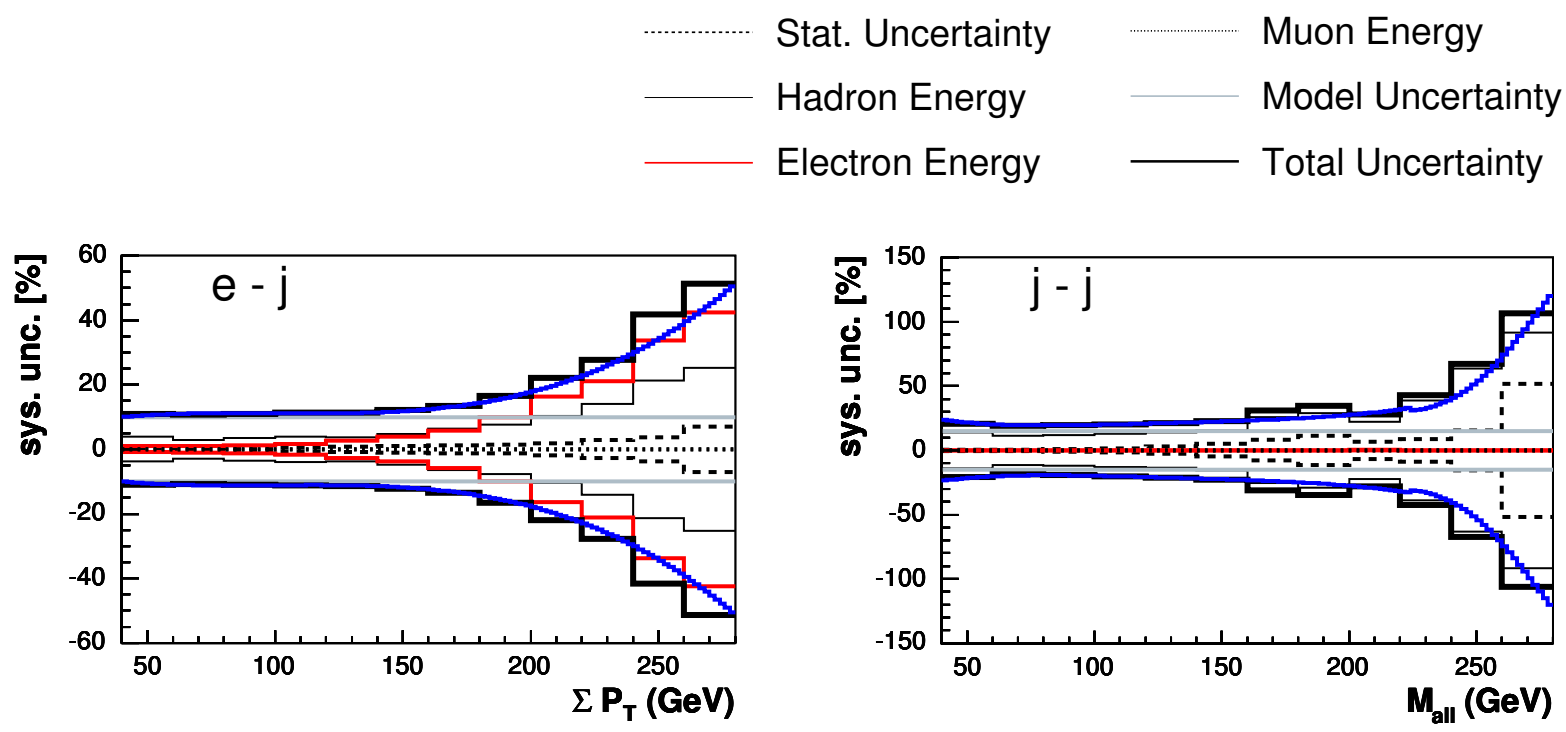

Fig. 5.10: The systematic uncertainty on the predicted number of events for the $e-j$ and $j-j$ event class as a function of the sum of transverse momenta $\sum P_{T}$ and the invariant mass $M_{\text {all }}$, respectively. Only the total uncertainty and the corresponding fit as well as the uncertainties arising from the energy measurement and the Monte Carlo statistics are shown.

highest values measured in data. In the $j$ - $j$ event class the error varies from $20 \%$ for low up to $50 \%$ for high invariant masses of around $250 \mathrm{GeV}$.

The distributions of the systematic uncertainties for all event classes containing data events are presented in appendix C.

\subsection{Multi-Jet Topologies}

The dominant production processes for multi-jet events in $e p$ interactions are NC and CC deep-inelastic scattering as well as photoproduction, including additional jet production due to higher order QCD processes.

The exact matrix elements are used for the simulation of the $\mathcal{O}\left(\alpha_{s}\right)$ processes, Boson Gluon Fusion and QCD Compton scattering, while further jets are produced using leading logarithmic parton showers or the colour dipole model approach. In order to adjust the normalisation of the leading order Monte Carlos to that of next-to-leading order QCD calculations the prediction of processes with two or more high transverse momentum jets is scaled by a factor of 1.2 (cf. section 3.1).

In the present analysis final states including four high transverse momentum jets are measured in the $j-j-j-j$ and $e-j-j-j-j$ event class, where the dominant SM contribution contains two jets produced via parton showers. An overshoot of the data compared to the $\mathrm{SM}$ prediction is found in both classes (cf. section 6). Since so far only up to three-jet 

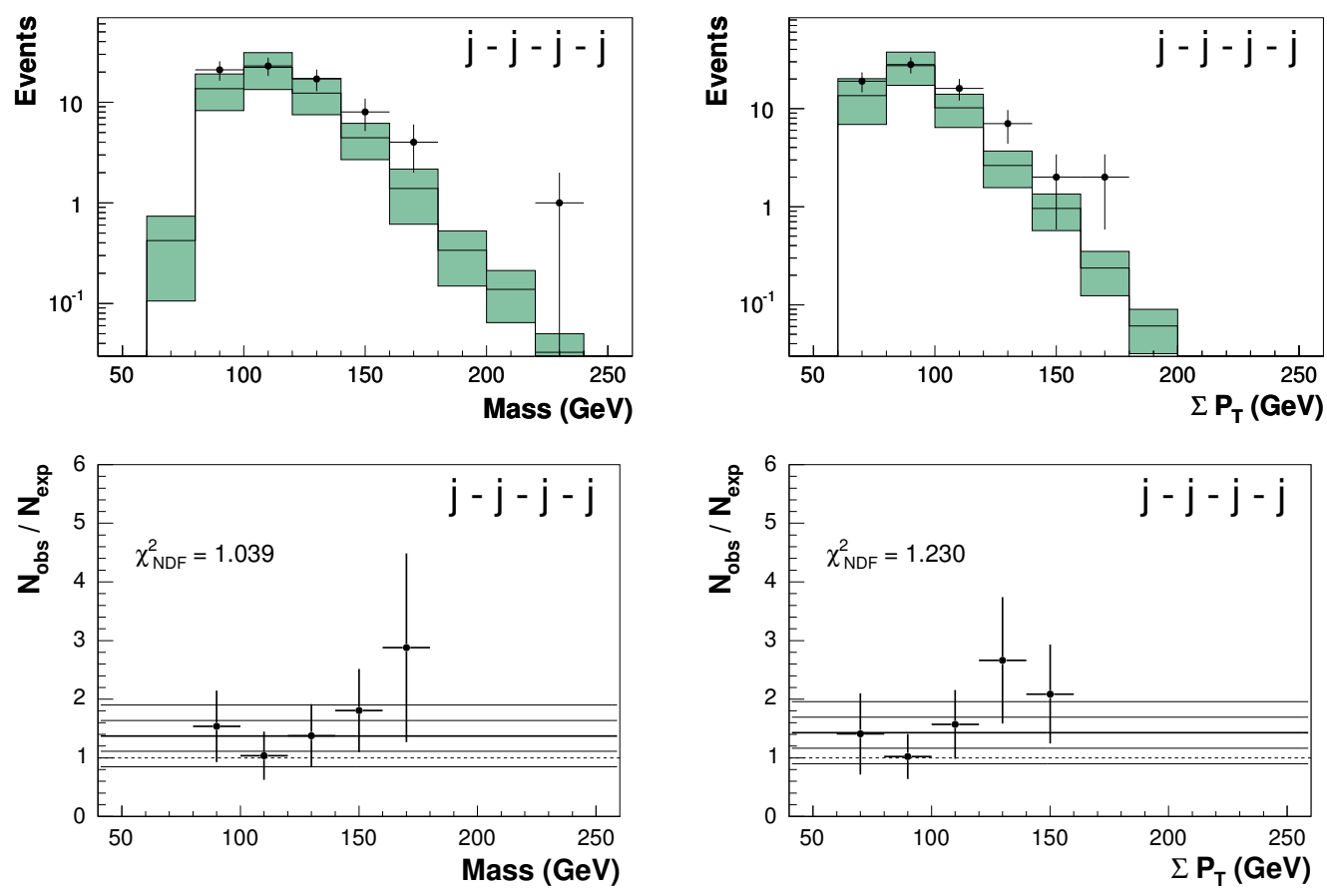

Fig. 5.11: The distributions of the invariant mass $M_{\text {all }}$ and the sum of transverse momenta $\sum P_{T}$ for the $j-j-j-j$ event class obtained from a low- $P_{T}$ jet sample (upper figures) and the ratio of the observed and expected number of events (lower figures). A constant function is fitted on the ratio distribution. Presented are the mean value as well as the regions containing one and two standard deviations.

cross sections have been published in photoproduction and neutral current DIS by the H1 collaboration $[19,59,60]$, further investigation on the feasibility of the measurement of those event topologies is performed.

The measured cross section for inclusive three-jet production in photoproduction has been investigated in [55]. The shape of the invariant three-jet mass distribution has been found to be well described by the rescaled prediction obtained from the event generator PYTHIA, even better than by leading order QCD calculations ${ }^{4}$.

Four-jet production in neutral current DIS events has been studied in [61] and the cross section as a function of the invariant four-jet mass has been compared to QCD calculations in leading order, i.e. $\mathcal{O}\left(\alpha_{s}^{3}\right)$. While the perturbative QCD prediction slightly overshoots the data, a good description has been found when hadronization corrections are considered.

In the present analysis the ability of the used leading order Monte Carlos to describe topologies including four high transverse momentum jets is investigated in neutral current DIS and photoproduction events. The distributions of the invariant mass and the

\footnotetext{
${ }^{4}$ Both the direct results of the $\mathcal{O}\left(\alpha_{s}^{2}\right)$ QCD calculation and its prediction including hadronization corrections have been considered.
} 
sum of transverse momenta are studied for both the $j-j-j-j$ and $e-j-j-j-j$ class, obtained from event samples with reduced transverse momentum requirements.

The SM prediction in the $j-j-j$ - $j$ class has been tested on events including at least one jet with $P_{T}>20 \mathrm{GeV}$ to ensure a high trigger efficiency, while the $P_{T}$ requirement on further jets is lowered to $15 \mathrm{GeV}$. In total 74 data events are measured compared to an expectation of $55.0 \pm 19.4$ events. Even though at the upper edge, the event yield is described within one standard deviation of the number predicted by the SM. The quoted error denotes the total systematic uncertainty in the expectation.

The obtained invariant mass and sum of transverse momenta distributions are presented in the upper part of figure 5.11. Both shape and normalisation are reasonably well described within the systematic error considered except for the highest $M_{\text {all }}$ and $\sum P_{T}$ bin, where a slight data overshoot is observed.

In order to quantify the level of agreement between the data and SM prediction, the according ratios of the observed and expected number of events as a function of $M_{\text {all }}$ and $\sum P_{T}$ are shown in the lower diagrams of figure 5.11. The error quoted on the values is obtained using the systematic uncertainty in the SM prediction only.

A constant function is fitted on the ratio distribution to estimate the overall normalisation, obtaining a value of $1.4 \pm 2.6$ for the invariant mass and $1.4 \pm 2.7$ for the sum of transverse momenta distribution, respectively.

The SM description of four-jet production in neutral current DIS has been tested using an exclusive event sample including one electron with $P_{T}>10 \mathrm{GeV}$ and four jets with $P_{T}>5 \mathrm{GeV}$. In total 169 data events are selected while 212.2 events are predicted from the SM for this event topology.

Figure 5.12 presents the transverse momentum and polar angle distributions of the second, third and forth jet, ordered by decreasing values of $P_{T}$. Both the normalisation and shape of all distributions are reasonably well described by the SM prediction.

The distributions of the invariant mass and the sum of transverse momenta for the selected events are shown in the upper diagrams of figure 5.13. The highest values measured in data amount to $262 \mathrm{GeV}$ for $M_{\text {all }}$ and $207 \mathrm{GeV}$ for $\sum P_{T}$. Both numbers arise from the same event, which is the only event selected in the $e-j-j-j-j$ event class of the final analysis.

In order to quantify the uncertainty of the SM prediction in this high $M_{\text {all }}$ and $\sum P_{T}$ region, a linear function is fitted on the distribution of the according ratios of the observed and expected number of events, presented in the lower diagrams of figure 5.13. The error quoted on the obtained ratios arises from the statistical error of the data measurement. A maximum value for the underestimation of the SM prediction is conservatively obtained by increasing both the slope and intercept of the fitted straight line by twice the according standard deviations at the same time. This variation would cause an approximately threefold SM expectation in the region of high invariant masses and high values of transverse momenta, respectively. 

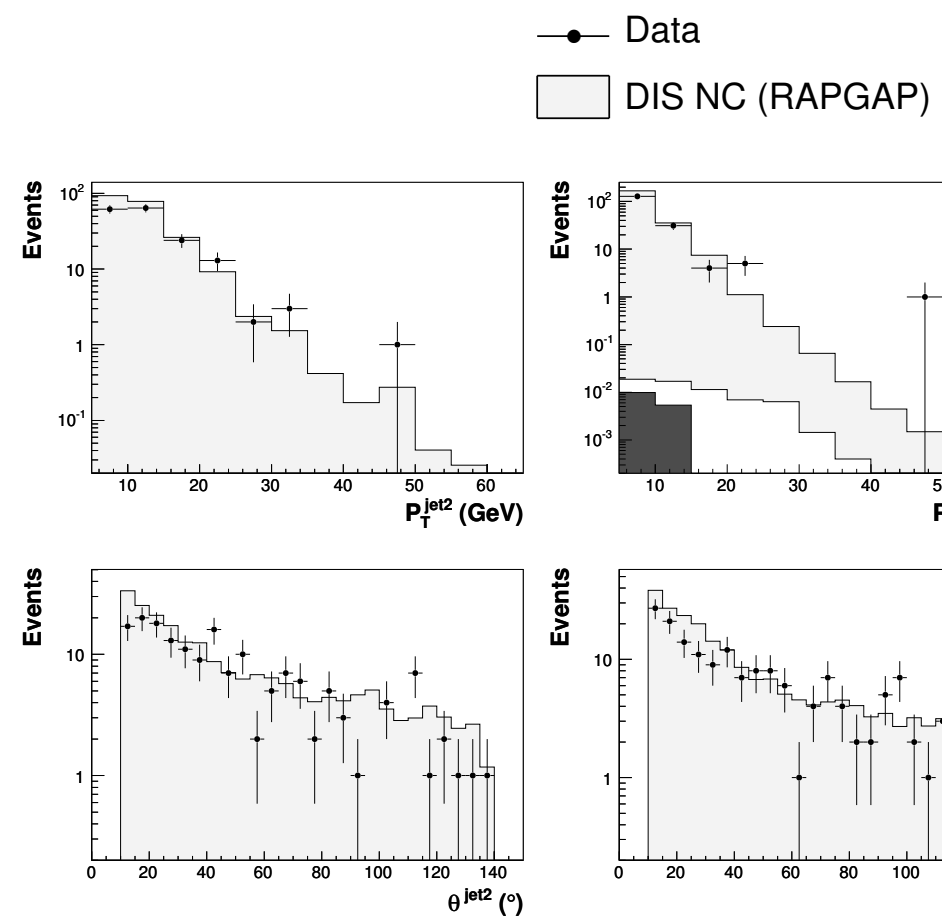
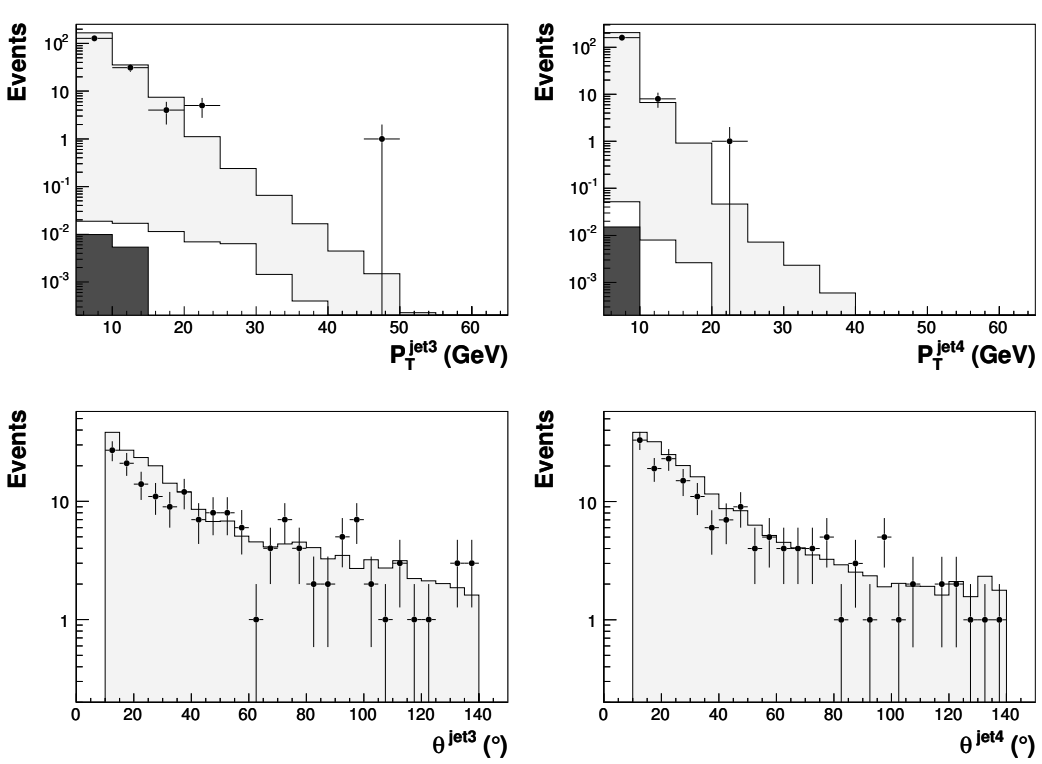

ee (GRAPE)

$W->q q^{\prime}(E P V E C)$

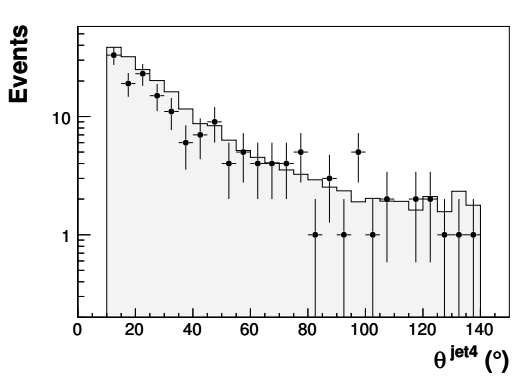

Fig. 5.12: The distributions of the transverse momentum $P_{T}$ and polar angle $\theta$ of the second, third and forth jet in a low- $P_{T} e-j-j-j-j$ event sample.
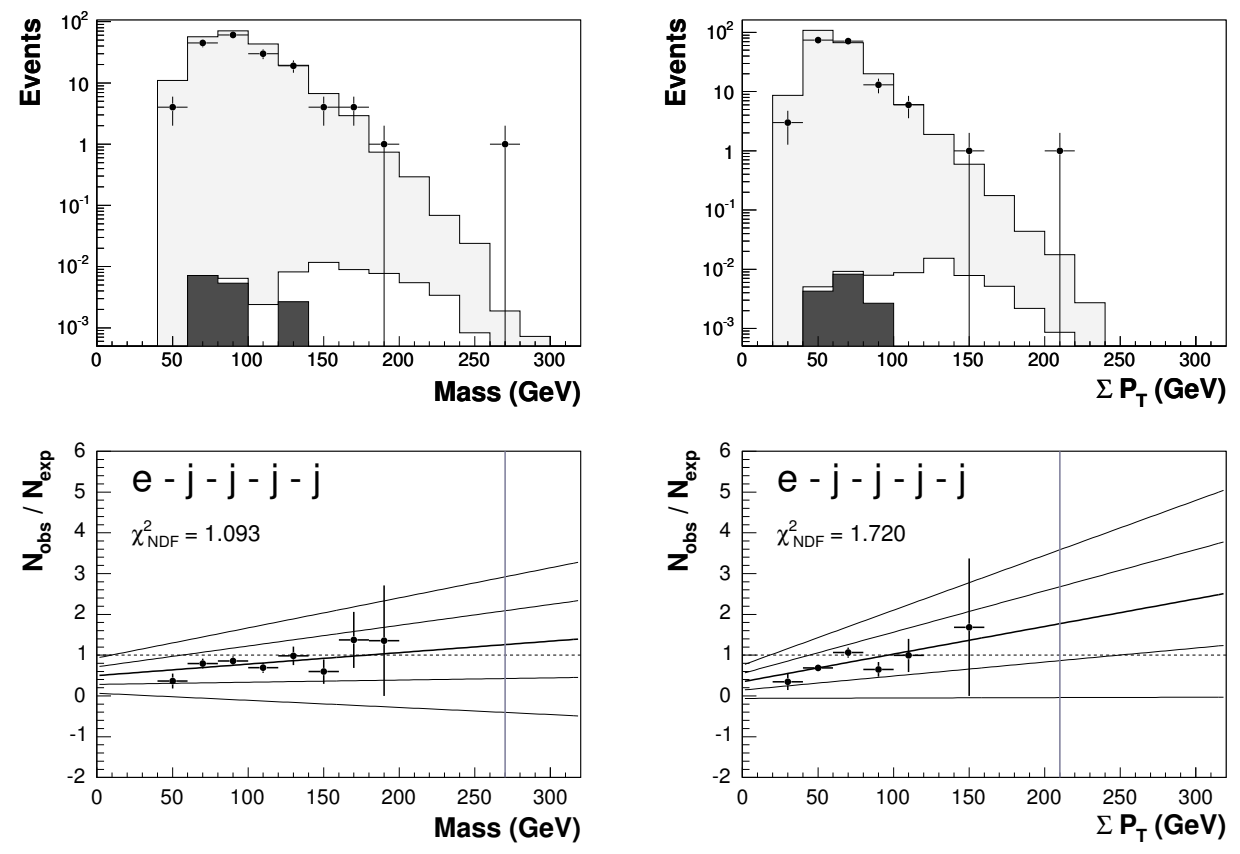

Fig. 5.13: The distributions of the invariant mass $M_{\text {all }}$ and the sum of transverse momenta $\sum P_{T}$ for the $e-j-j-j-j$ event class obtained from a low- $P_{T}$ event sample (upper figures) and the ratio of the observed and expected number of events (lower figures). A linear function is fitted on the ratio distribution. Presented are the mean value as well as the regions containing one and two standard deviations. The vertical lines denote the highest values of $M_{\text {all }}$ and $\sum P_{T}$ observed in the data. 


\section{Chapter 6}

\section{Experimental Results}

For the first time all possible final state topologies with at least two high transverse momentum particles have been investigated in a coherent way. Based on the complete HERA-I data sample the obtained event yields subdivided into event classes are presented for the data and SM expectation in figure 6.1. Shown are all classes with a SM expectation greater than 0.01 events $^{1}$. In total 23 event classes containing data events have been found, which is in agreement with the prediction of the SM. As discussed in section 5.4 the $\mu-\nu$ event class has been discarded from further investigations as its SM prediction is overwhelmed by imprecisely known low- $P_{T}$ processes.

The distributions of the invariant mass $M_{\text {all }}$ and the scalar sum of transverse momenta $\sum P_{T}$ are derived for all event classes and will be discussed in extracts within this section. All distributions are systematically researched for the appearance of new physics signals in chapter 7 and are presented in the figures 7.9 and 7.10 .

The following paragraphs discuss the results obtained from the measurement of the individual event topologies. A good agreement between data and the SM expectation is observed for most of the event classes in both the resulting event yields as well as the distributions of $M_{\text {all }}$ and $\sum P_{T}$, representing the good understanding of SM physics up to the borders of phase space.

\section{Dominant Processes}

The dominant processes at HERA, which are the neutral and charged current deepinelastic scattering as well as the photoproduction of jets, manifest themselves in the $e-j, j-\nu$ and $j-j$ event class, respectively. The event yields of those classes are the highest measured in the analysis and in good agreement with the SM expectation.

Figure 6.2 presents the invariant mass distributions obtained. A good description of the data spectra is observed for all event classes. Both the $M_{\text {all }}$ and $\sum P_{T}$ values of the data reach up to values of $250 \mathrm{GeV}$ (cf. figure 4.14). These are the highest values investigated for di-jet events in ep collisions so far.

\footnotetext{
${ }^{1}$ In none of the event classes populated by data a SM expectation smaller than 0.01 events is found.
} 


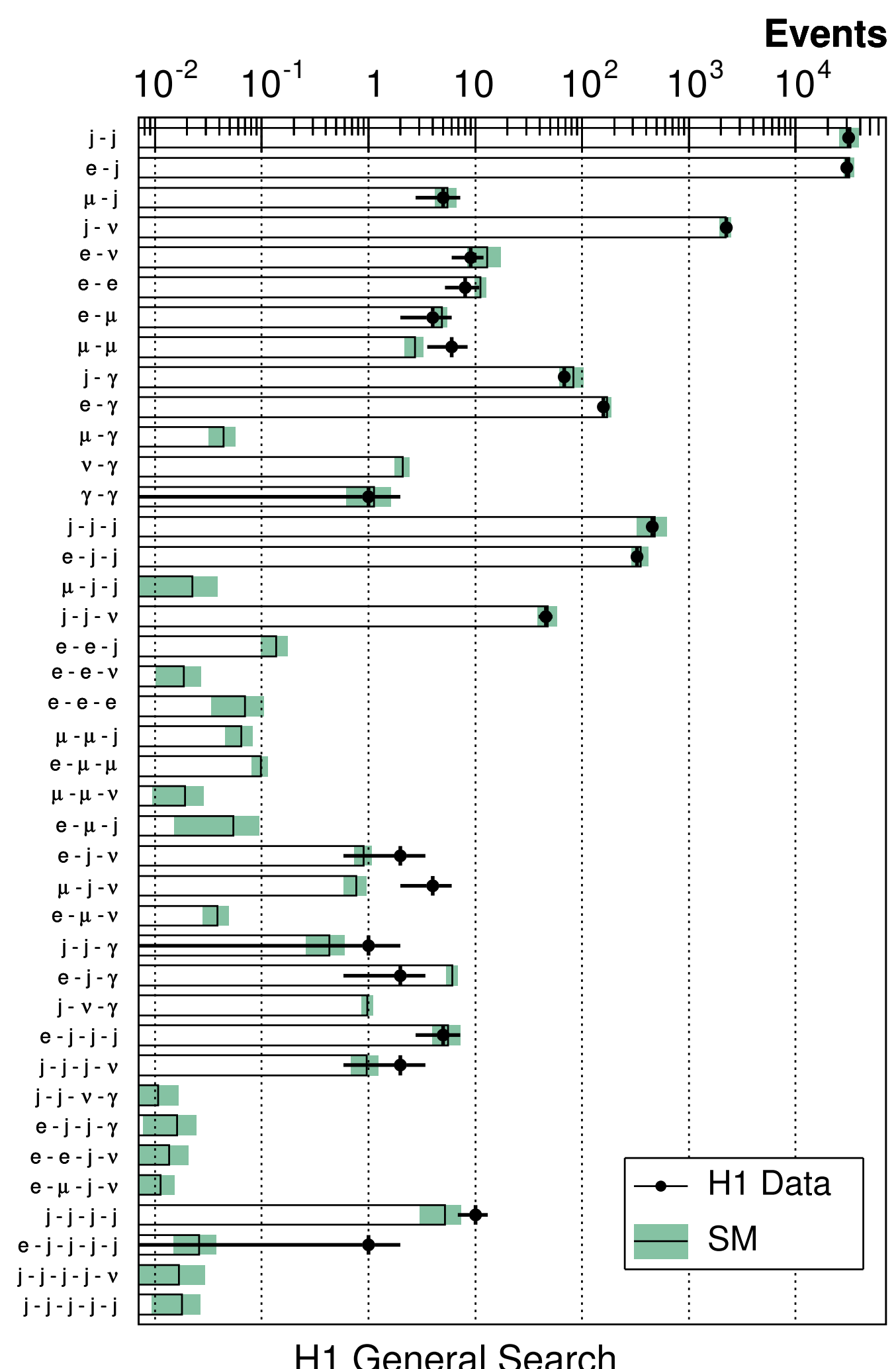

Fig. 6.1: The data and the SM expectation for all event classes with a SM expectation greater than 0.01 events as derived from the investigation of the complete HERA-I data sample. 

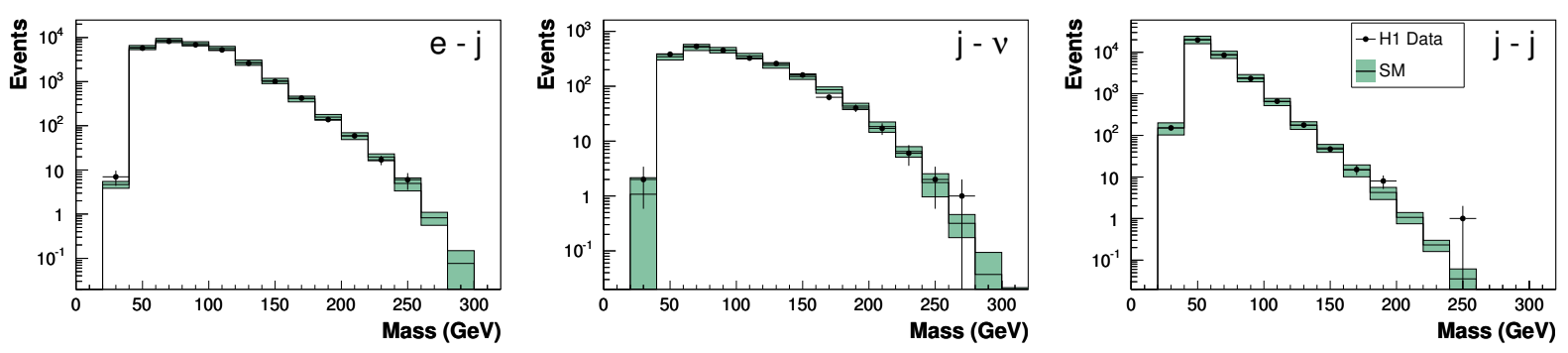

Fig. 6.2: The distributions of the invariant mass $M_{\text {all }}$ for the $e-j, j-\nu$ and $j$ - $j$ event classes.

\section{Photon Classes}

The $e-\gamma$ event class is dominated by events arising from QED Compton scattering processes. In good agreement with the SM expectation values of up to $160 \mathrm{GeV}$ are measured for the invariant mass and sum of transverse momenta.

No event is observed in the $\nu-\gamma$ and $j-\nu-\gamma$ event classes, consistent with the expectation of $2.1 \pm 0.3$ and $1.0 \pm 0.1$ events, respectively.

The $j$ - $\gamma$ event class is well described by the prediction of the SM, where invariant mass and sum of transverse momenta values of up to $160 \mathrm{GeV}$ are measured in the data. In total 68 data events are found compared to an expectation of $82.6 \pm 21.4$ events.

One event is observed in the $\gamma-\gamma$ event class for an expectation of $1.1 \pm 0.5$ events, which is dominated by radiative neutral current DIS processes, where the electron is misidentified because of cluster-track association inefficiency. Contributions of higher order QED processes, which could lead to two high transverse momentum photons, are not considered. A naive count of orders in the electromagnetic coupling $\alpha$ leads to an expectation of less than one event.

Further event classes containing photons are $j-j-\gamma$ and $e-j-\gamma$, which correspond to photoproduction and neutral current DIS processes including the radiation of a photon. In agreement with the SM prediction only 1 and 2 events are measured in these event classes, respectively.

\section{Lepton Pair Production}

A discrepancy from the SM expectation has been reported by the $\mathrm{H} 1$ collaboration in multi-electron events [38]. In the present analysis the $e$-e event class is populated to $85 \%$ by events arising from electron pair production. In total 8 events are measured in the data compared to a predicted number of $11.2 \pm 1.4$ events. All multi-electron events mentioned in [38] and located in the phase space of this analysis are found. No tri-electron event is identified due to the requirement of high transverse momentum.

The upper left diagram of figure 6.3 presents the distribution of the invariant mass for the $e$ - $e$ event class. In the region $M_{\text {all }}>100 \mathrm{GeV}, 3$ events are observed while $1.16 \pm 0.25$ events are expected. The higher SM prediction for this high mass region compared to the prediction of $0.3 \pm 0.04$ events given in [38] is caused by the extension of the phase 

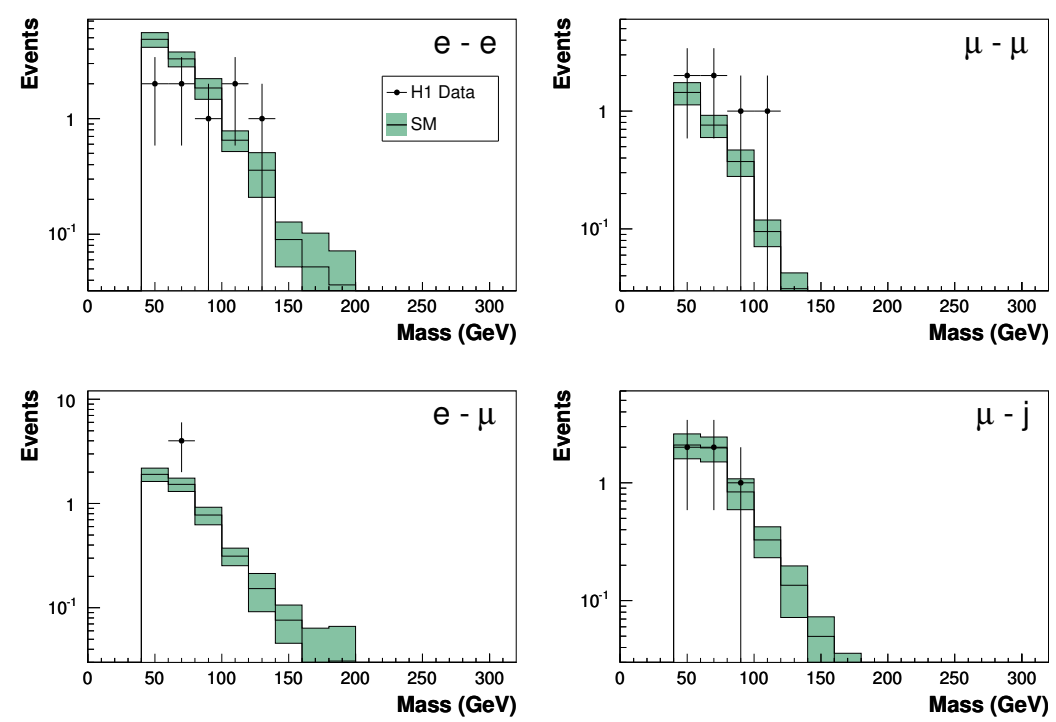

Fig. 6.3: The distributions of the invariant mass $M_{\text {all }}$ for the $e-e, \mu-\mu, e-\mu$ and $\mu-j$ event classes.

space to the very forward polar angle region in the presented analysis. On the one hand about 0.4 additional background events arise from the misidentification of electrons with $\theta<20^{\circ}$, while also the predicted contribution of signal events from lepton pair production processes is increased by roughly the same number. No additional data event is measured in the $e-e$ event class due to the extension of the phase space.

The $e-\mu$ and $\mu-\mu$ event classes are dominated to $97 \%$ and $100 \%$ by muon pair production events arising from two-photon interactions. The $e-\mu$ event class is populated by those events where the scattered electron but only one of the muons is selected. In the $e-\mu$ and $\mu-\mu$ event classes, 4 and 6 events are observed compared to a SM expectation of $4.8 \pm 0.6$ and $2.7 \pm 0.6$ events, respectively.

Muon pair production processes also contribute to $85 \%$ to the $\mu-j$ event class. Both the measured $\sum P_{T}$ and $M_{\text {all }}$ values of those classes range from 50 to $100 \mathrm{GeV}$, well described by the SM expectation. The distributions of the invariant mass are exemplarily presented in figure 6.3 .

\section{$W$ Production}

A discrepancy between the data and SM expectation is observed in the $\mu-j-\nu$ event class, where 4 events are observed compared to an expectation of $0.8 \pm 0.2$ events. A similar deviation was previously reported in [37] and will be further discussed in chapter 7.4.

The obtained distributions of the sum of transverse momenta and the invariant mass are presented in figure 6.4. The $\sum P_{T}$ values of the data reach $170 \mathrm{GeV}$ while values up to $200 \mathrm{GeV}$ are measured for $M_{\text {all }}$.

In the SM, event topologies including a high transverse momentum muon, jet and neu- 

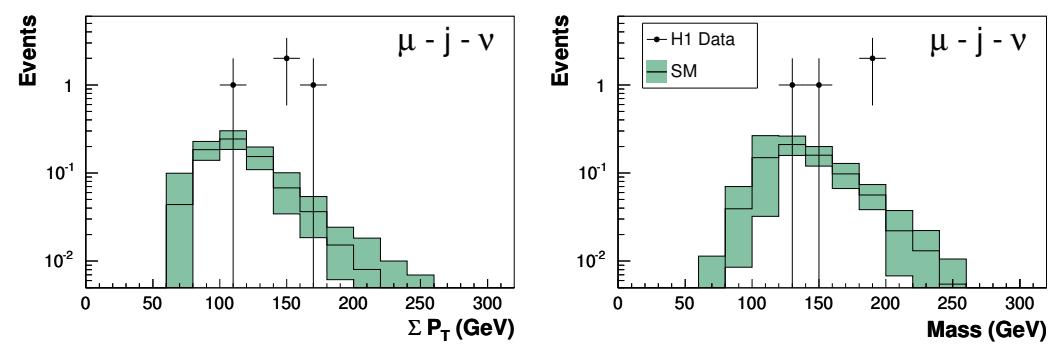

Fig. 6.4: The distributions of the sum of transverse momenta $\sum P_{T}$ and invariant mass $M_{\text {all }}$ for the $\mu-j-\nu$ event class.

trino correspond to $W$ production with a subsequent leptonic decay. While the $\mu-\nu$ event class was found to be overwhelmed by low- $P_{T}$ photoproduction background (cf. section 5.3.2) less than 0.002 events arising from photoproduction processes are expected for the $\mu-j-\nu$ class.

The event classes $e-\nu$ and $e-j-\nu$ are also populated by events arising from $W$ production. In the $e-\nu$ event class, 9 data events compared to an expectation of $12.9 \pm 4.5$ events are observed. This event class is dominated by background events from NC deep-inelastic scattering processes, where possible fluctuations in the hadronic energy measurement or limited detector acceptance can produce missing transverse momentum (cf. section 5.3.1). In the $e-j-\nu$ event class 2 data events are observed for an expectation of $0.9 \pm 0.2$ events. Some of the $e-j-\nu$ events mentioned in [37] contain an electron with a transverse momentum below $20 \mathrm{GeV}$ and are therefore not selected in the present analysis.

\section{Multi-Jet Topologies}

The $e-j-j$ and $j-j-\nu$ as well as the $e-j-j-j, j-j-j-\nu$ and $j-j-j$ event classes originate from the dominant $\mathrm{NC}$ and $\mathrm{CC}$ deep-inelastic scattering as well as photoproduction processes with the inclusion of additional jet production due to higher order QCD processes. Both the event yields and distributions of the invariant mass and sum of transverse momenta for those classes are well described by the SM prediction. While the exact matrix elements are used for the simulation of $e-j-j$ and $j-j-\nu$ events arising from NC and CC DIS processes, one jet produced by parton showers or using the dipole approach, respectively, is contained in the SM expectation of the $e-j-j-j, j-j-j-\nu$ and $j-j-j$ event classes. Figure 6.5 exemplarily presents the sum of transverse momenta distributions obtained.

Events with four high transverse momentum jets are investigated for the first time at HERA. The dominant SM contribution to both classes, i.e. photoproduction and neutral current DIS, respectively, contains two jets mainly produced via parton showers.

A slight overshoot of data compared to the SM prediction is observed in the $j-j-j-j$ event class with 10 data events, whereas $5.2 \pm 2.2$ events are expected. The contribution of photoproduction events to the SM expectation amounts to $85 \%$, while the remaining fraction arises from NC DIS processes with a low- $P_{T}$ electron. The obtained $M_{\text {all }}$ and $\sum P_{T}$ dis- 

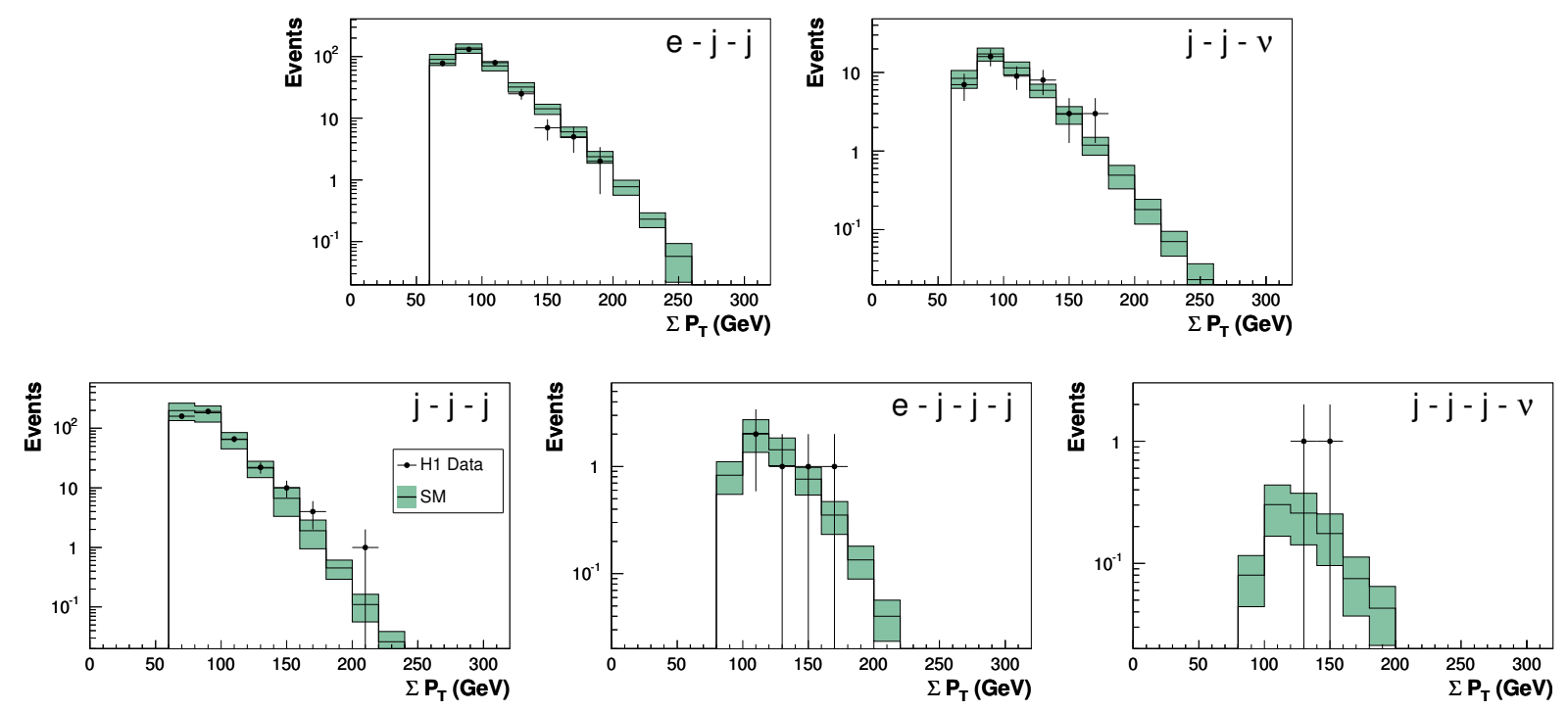

Fig. 6.5: The distributions of the sum of transverse momenta $\sum P_{T}$ for the $e-j-j$ and $j-j-\nu$ as well as $j-j-j, e-j-j-j$ and $j-j-j-\nu$ event classes.

tribution are presented in the upper diagrams of figure 6.6.

The one event observed in the $e-j-j-j-j$ class has to be compared to an expectation of $0.026 \pm 0.011$ events. For this interesting event the sum of transverse momenta amounts to $207 \mathrm{GeV}$ and a very high invariant mass of about $262 \mathrm{GeV}$ is reconstructed. The latter is particularly remarkable since the event originates from a data taking period with a proton momentum of $820 \mathrm{GeV}$, resulting in an available centre-of-mass energy of $301 \mathrm{GeV}$. The neutral current DIS expectation in the corresponding invariant mass region $M_{\text {all }}>260 \mathrm{GeV}$ amounts to $(9 \pm 6) \cdot 10^{-5}$ events.

Both the distributions of $M_{\text {all }}$ and $\sum P_{T}$ for the $e-j-j-j-j$ event class are shown in the lower diagrams of figure 6.6. A display of the data event is presented in figure D.1.

In order to verify the authenticity of the SM prediction for events including two jets produced using the parton shower model, a low- $P_{T}$ four-jet as well as an electron-four-jet sample has been studied in section 5.7. A consistent description of the shapes of the $M_{\text {all }}$ and $\sum P_{T}$ distributions of the data within the quoted SM uncertainties is observed in both samples. Likewise leading order $\mathrm{QCD} \mathcal{O}\left(\alpha_{s}\right)$ parton shower Monte Carlo generators have been found to describe four jets production in $p \bar{p}$ collisions [62] and in the $Z^{0}$ data collected in $e^{+} e^{-}$annihilations [63, 64]. Furthermore the $\sum P_{T}$ and $M_{\text {all }}$ distributions of tri-jet event topologies are well described by the SM prediction within the parton shower or dipole model approach up to the observed sum of transverse momenta and invariant mass values of $200 \mathrm{GeV}$ (cf. figure 6.5).

All of those studies militate in favour, that the SM prediction derived using leading order parton-shower Monte Carlo programs is sufficiently modelled in order to describe events with four high transverse momentum jets arising from neutral current DIS or photopro- 

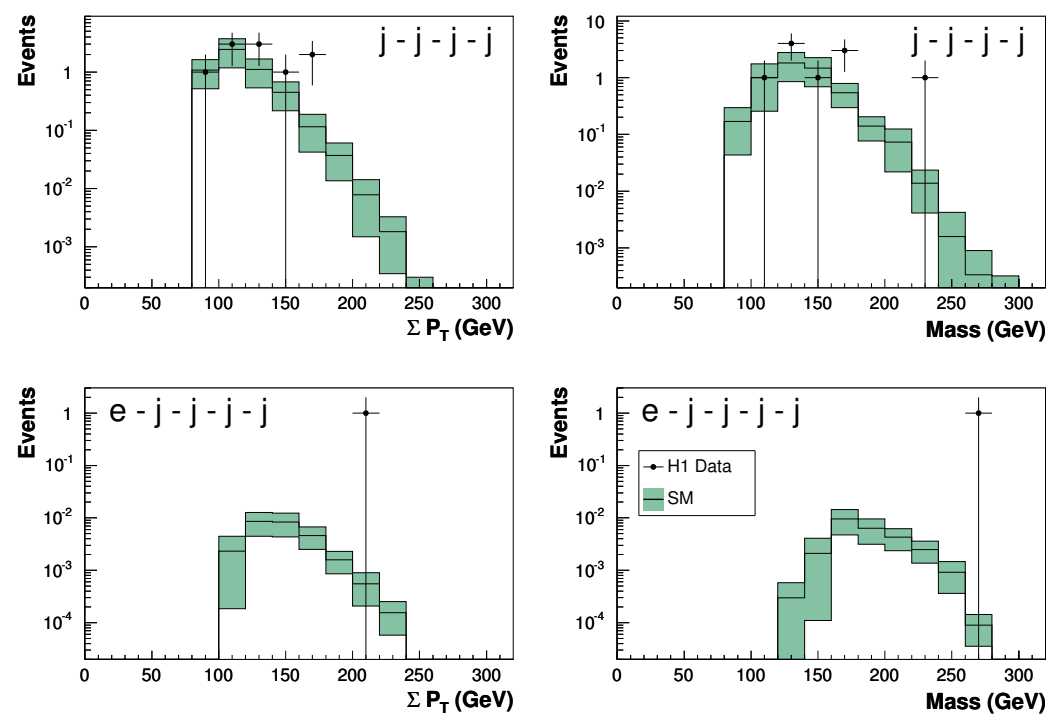

Fig. 6.6: The distributions of the sum of transverse momenta $\sum P_{T}$ and invariant mass $M_{\text {all }}$ for the $j-j-j-j$ and $e-j-j-j-j$ event classes.

duction processes. Anyhow, these dominant processes yield only about $10^{-4}$ events in the regions of very high sum of transverse momenta or invariant masses, corresponding to a cross section in the order of $0.001 \mathrm{fb}$ (cf. figure 6.6). Therefore rare SM processes not considered in this analysis might contribute in these kinematic domains. An example is the production of $W$ pairs with a subsequent decay into four jets. For that reason, the results obtained from the search algorithm in chapter 7 for the $j-j-j-j$ and $e-j-j-j-j$ event classes have to be interpreted with more attention.

The observed number of events compared to the number of events predicted by the SM is given in table D.1 for all event classes. The individual contributions to the SM expectation arising from the different physical processes are listed in table D.2.

The results discussed above are based on the complete HERA-I data collected during the years 1994 to 2000. In order to test the dependence on combining the data from positron-proton collisions with that obtained from colliding electrons on protons between 1998 and midyear 1999, the analysis is repeated on both data samples separately.

Figure 6.7 presents the event yields subdivided into the event classes for the $e^{+} p$ and $e^{-} p$ data sample. Shown are the same event classes as for the complete HERA-I data sample, in order to compare the derived results.

A similar agreement between the data and SM prediction is found also for the particular data samples. No new deviation is observed in any further event class. According to the small luminosity of the electron sample the rates of the individual event classes are reduced significantly. All interesting events populating the $\mu-j-\nu$ event class as well as the single $e-j-j-j-j$ event originate from the $e^{+} p$ data sample. 

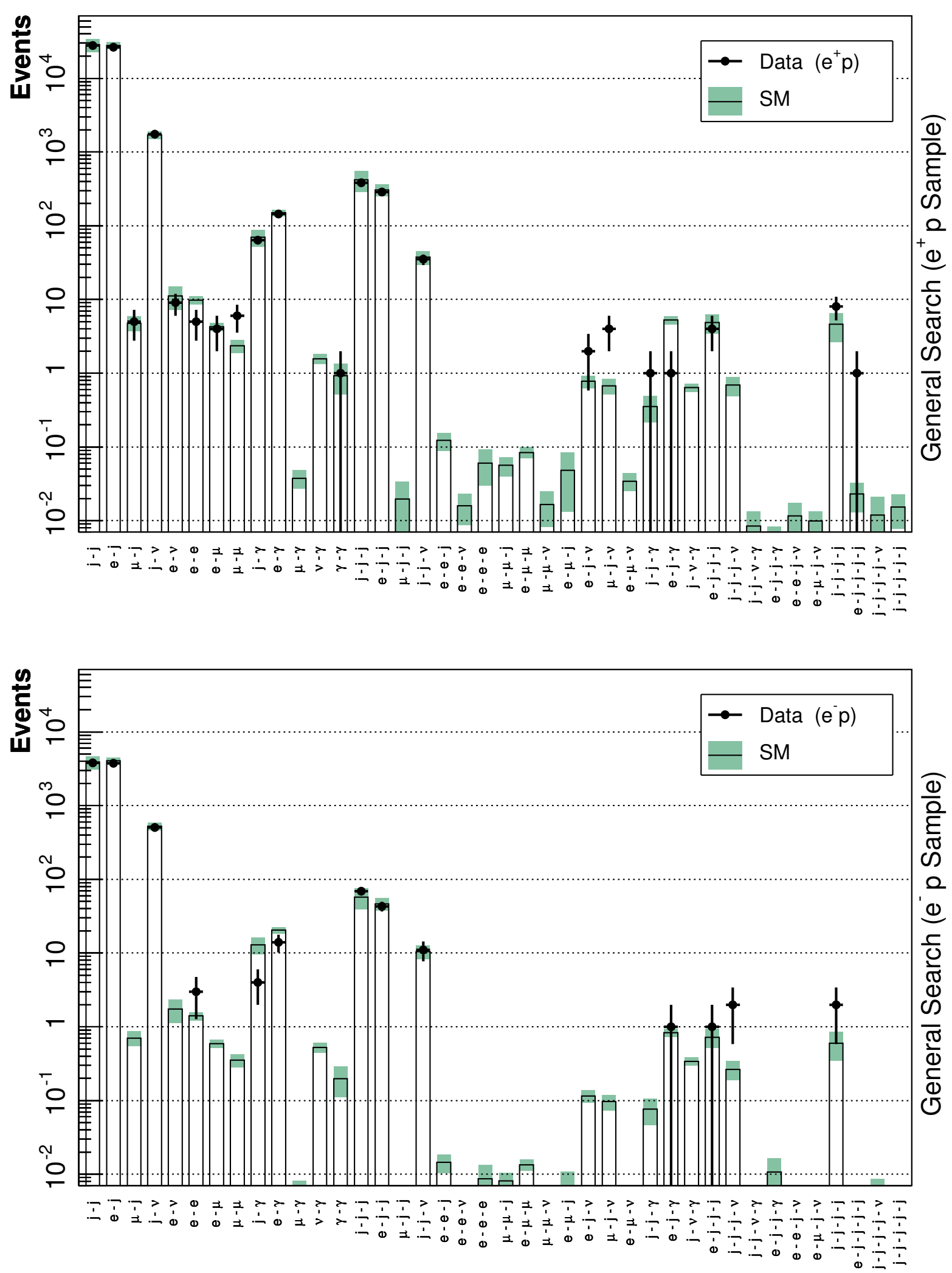

Fig. 6.7: The event yields subdivided into event classes as derived from the investigation of the $e^{+} p$ (upper figure) and $e^{-} p$ (lower figure) data samples. Shown are all event classes with a SM expectation greater than 0.01 events for the full HERA-I data sample. 
In order to test the dependence of the analysis on the a priori defined $P_{T}$ cut of $20 \mathrm{GeV}$ for all considered objects the whole analysis is repeated with two other requirements on the transverse momentum. The $P_{T}$ cut has been raised to $40 \mathrm{GeV}$ for all objects and lowered to $15 \mathrm{GeV}$ except one to ensure a high trigger efficiency (cf. section 5.2).

The number of observed data events and the SM expectation for all event classes with a SM expectation greater than 0.01 events as derived from the investigation of these control samples are shown in figure 6.8. A similar agreement with the SM prediction as in the results obtained from the central analysis is found for both samples.

In the scenario with the lowered $P_{T}$ cut again a discrepancy is observed in the $\mu-j-\nu$ event class, where 6 events are measured while only $1.3 \pm 0.3$ are predicted by the SM. The $e-j-j-j-j$ event class remains populated by only the one event already found in the central analysis, now to be compared to a SM expectation of $0.6 \pm 0.2$ events.

Three new event classes are found to be populated by data. In each, one data event is measured in the $e-e-j$ and $j-j-j-j-j$ event class, while 2 events are observed in the $j-\nu-\gamma$ class. The yields of all three classes are well in agreement with the SM expectation. For the event sample with the transverse momentum requirement on the objects raised to $40 \mathrm{GeV}$ it is mainly the two particle event classes containing jets which are still populated by events. As a result of the though $P_{T}$ cut no events are measured anymore in the $\mu-j-\nu$ event class, while the excess of di-electron events already reported in [38] is exposed. In the $e$-e event class 3 data events are measured compared to an SM expectation of $0.8 \pm 0.1$ events. No new deviation is found also for this scenario. 

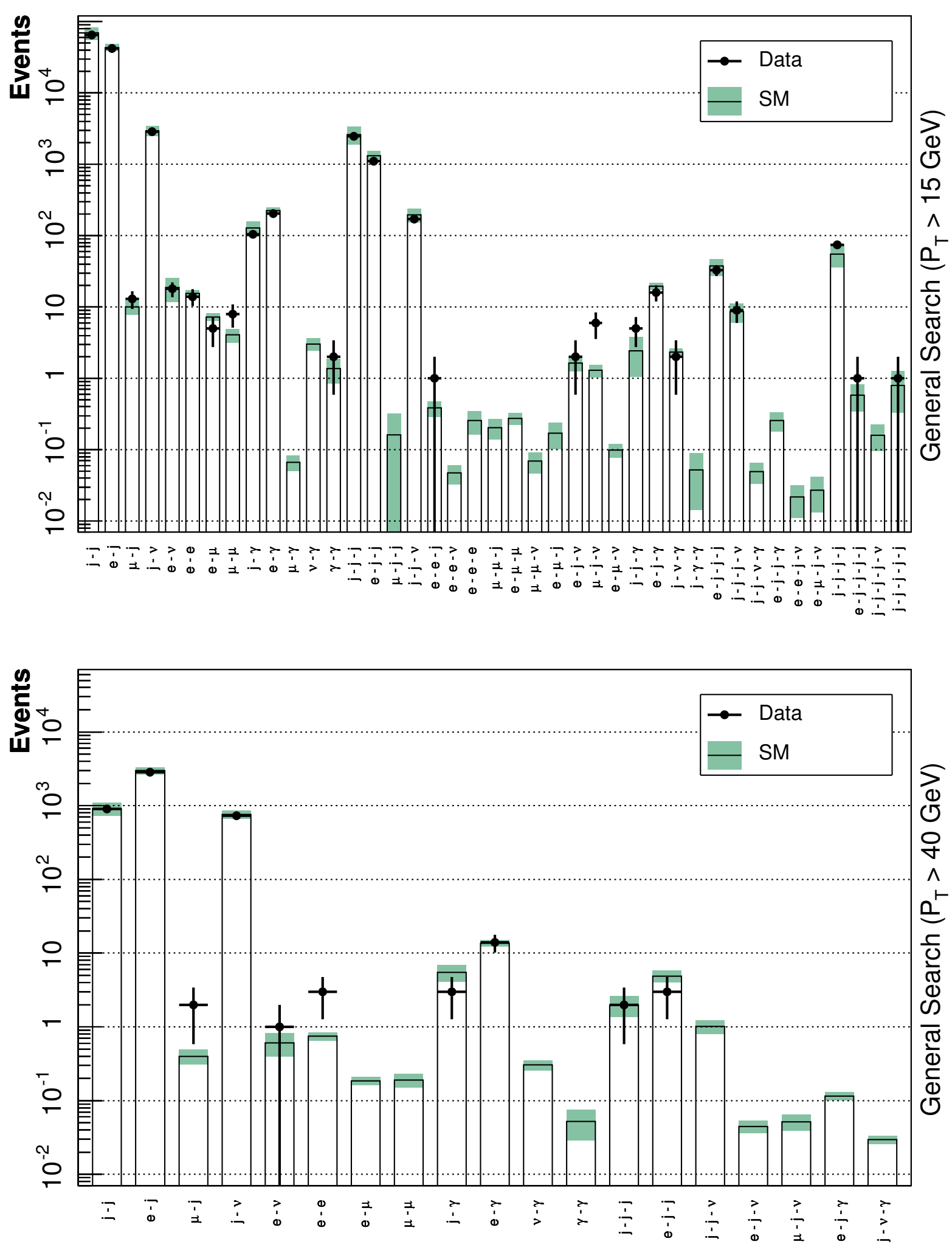

Fig. 6.8: The data and SM expectation for all event classes with a SM expectation greater than 0.01 events as derived from the investigation of the event samples with the $P_{T}$ cut lowered to $15 \mathrm{GeV}$ (upper figure) and raised to $40 \mathrm{GeV}$ (lower figure). 


\section{Chapter 7}

\section{Search for Deviations}

In order to search for signals of new physics the obtained distributions of the invariant mass and the sum of transverse momenta are systematically investigated for all event classes within the framework of a statistical analysis. A new search algorithm has been developed which identifies the regions of possible deviations and allows for a quantitative determination of the level of agreement between the data and SM expectation. It is based on basic principles of statistics, no complex mathematical theories are involved.

The statistical algorithm is introduced in detail in the first part of the following chapter. Subsequently studies on the sensitivity to new physics are presented, for which various hypothetical signals have been mixed with the SM expectation. Finally, the search results obtained from the investigation of all event classes are discussed.

\subsection{Search Algorithm}

The algorithm described in the following locates the region of largest deviation between the data and the SM prediction in a given one-dimensional histogram, independent of the shapes of the underlying distributions. Since detailed studies have shown that both the sum of transverse momenta $\sum P_{T}$ and the invariant mass $M_{\text {all }}$ of all objects in an event class are sensitive to new physics signals at large energies (cf. section 7.3), the distributions of these quantities have been chosen for investigation in this analysis.

In order to quantify the significance of the deviations found by the algorithm in the $\sum P_{T}$ and $M_{\text {all }}$ distributions of an individual event class, the probability of occurrence of such a deviation in the according distribution is calculated. The calculation of the significance of a deviation is inspired by $[65,66]$.

\section{Definition of Regions}

The search algorithm is based on the appropriate definition of regions in order to locate the largest deviation between the data and SM expectation in the investigated histograms of the invariant mass and sum of transverse momenta distributions. A region is defined by a number of connected histogram bins and is required to have a size in the order of 


\begin{tabular}{|c|c|c||c|c|c|}
\hline event class & $\begin{array}{c}\Delta\left(\sum P_{T}\right) \\
(\mathrm{GeV})\end{array}$ & $\begin{array}{c}\Delta\left(M_{\text {all }}\right) \\
(\mathrm{GeV})\end{array}$ & event class & $\begin{array}{c}\Delta\left(\sum P_{T}\right) \\
(\mathrm{GeV})\end{array}$ & $\begin{array}{c}\Delta\left(M_{\text {all }}\right) \\
(\mathrm{GeV})\end{array}$ \\
\hline$j-j$ & 20 & 20 & $j-j-j$ & 20 & 20 \\
$e-j$ & 20 & 20 & $e-j-j$ & 20 & 20 \\
$\mu-j$ & 15 & 15 & $j-j-\nu$ & 20 & 20 \\
$j-\nu$ & 20 & 20 & $e-j-\nu$ & 20 & 20 \\
$e-\nu$ & 15 & 15 & $\mu-j-\nu$ & 20 & 20 \\
$e-e$ & 10 & 10 & $j-j-\gamma$ & 20 & 20 \\
$e-\mu$ & 10 & 10 & $e-j-\gamma$ & 20 & 20 \\
$\mu-\mu$ & 20 & 20 & $j-j-j-j$ & 20 & 20 \\
$j-\gamma$ & 20 & 20 & $e-j-j-j$ & 20 & 20 \\
$e-\gamma$ & 10 & 10 & $j-j-j-\nu$ & 20 & 20 \\
$\gamma-\gamma$ & 10 & 10 & $e-j-j-j-j$ & 20 & 20 \\
\hline
\end{tabular}

Tab. 7.1: The minimal region size $\Delta$ considered by the search algorithm in the investigation of the $\sum P_{T}$ and $M_{\text {all }}$ distributions for all event classes containing data events.

twice the resolution of the researched quantity.

The resolutions of the sum of transverse momenta and invariant mass have been determined for all event classes in section 5.4. Though a slight variation of the resolutions as a function of $\sum P_{T}$ and $M_{\text {all }}$ has been noticed, the consideration of fixed values used by the search algorithm is found to be a sufficient approximation. Depending on the individual event classes the obtained resolutions vary between 5 and $15 \mathrm{GeV}$. Based on these values and the bin size of the underlying histograms the minimal size $\Delta$ of the regions used by the search algorithm is chosen for all event classes as presented in table 7.1.

In order to minimise binning effects, a bin size smaller than the resolution of the researched quantities is used. A histogram graduation in $5 \mathrm{GeV}$ bins is chosen for both the distributions of the invariant mass and sum of transverse momenta in all event classes. Studies using histogram binnings of 1 and $2 \mathrm{GeV}$ have shown, that a further reduction of the bin size has only a negligible effect on the results.

All possible regions of any width and at any position in the histograms are considered by the search algorithm. The number of observed data events $N_{\text {obs }}$, the SM expectation $N_{S M}$ and its total uncertainty $\delta N_{S M}$ are calculated in each region in order to determine the level of agreement between the data and the SM prediction in the considered region and to select the region of most interest.

\section{Probability Calculation}

For each considered region the probability is calculated, that the number of events $N_{S M}$ predicted by the SM fluctuates upwards $\left(N_{o b s} \geq N_{S M}\right)$ or downwards $\left(N_{o b s}<N_{S M}\right)$ to the observed number of data events $N_{o b s}$. Therefore a statistical estimator $p$ is defined which is derived from the convolution of a Poisson probability density function (pdf) with 

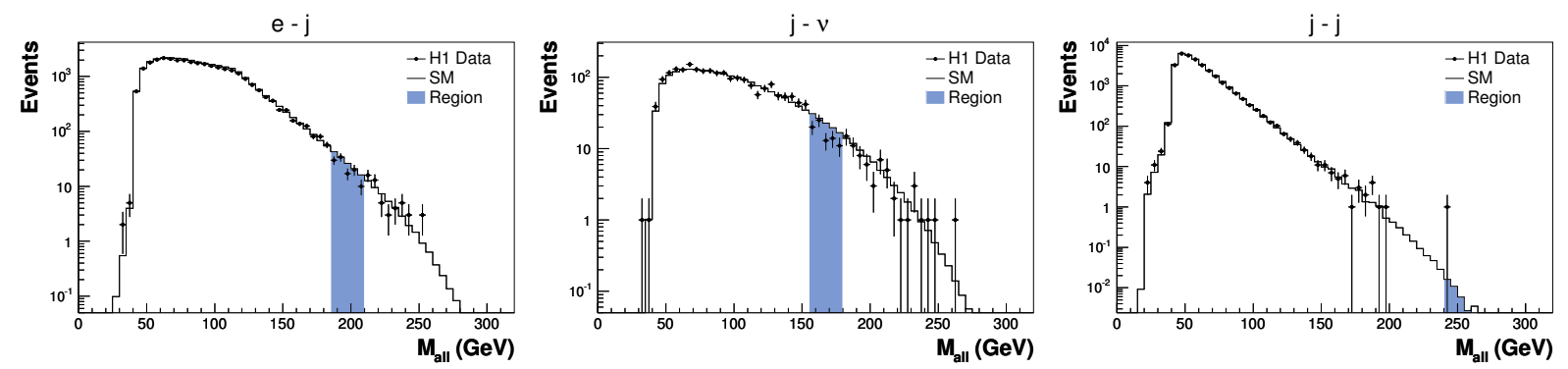

Fig. 7.1: The distributions of the invariant mass $M_{\text {all }}$ including the regions of greatest deviation chosen by the search algorithm for the $e-j, j-\nu$ and $j-j$ event classes.

a Gaussian pdf $G\left(b, N_{S M}, \delta N_{S M}\right)$ with mean $N_{S M}$ and width $\delta N_{S M}$. While the Poisson component of the estimator accounts for the statistical error, the Gaussian part includes the non negligible effect of systematic uncertainties. The estimator $p$ is defined by

$$
\begin{aligned}
& p=\left\{\begin{array}{lll}
A \int_{0}^{\infty} d b G\left(b, N_{S M}, \delta N_{S M}\right) & \sum_{i=N_{o b s}}^{\infty} \frac{e^{-b} b^{i}}{i !} & \text { if } N_{o b s} \geq N_{S M} \\
A \int_{0}^{\infty} d b G\left(b, N_{S M}, \delta N_{S M}\right) & \sum_{i=0}^{N_{o b s}} \frac{e^{-b} b^{i}}{i !} & \text { if } N_{o b s}<N_{S M}
\end{array}\right. \\
& \text { with } A=1 /\left[\int_{0}^{\infty} d b G\left(b, N_{S M}, \delta N_{S M}\right) \sum_{i=0}^{\infty} \frac{e^{-b} b^{i}}{i !}\right] \text {. }
\end{aligned}
$$

The factor $A$ ensures the normalisation to unity. If the Gaussian pdf $G\left(b, N_{S M}, \delta N_{S M}\right)$ is replaced by a Dirac delta function $\delta\left(b-N_{S M}\right)$ the estimator $p$ becomes the usual Poisson probability for observing $N_{o b s}$ events with a mean rate of $N_{S M}$ events.

\section{Regions of Most Interest}

A possible sign of new physics is found if the expectation significantly disagrees with the data. The level of agreement between the data and SM expectation is quantified by the estimator $p$ for each considered region of the researched $\sum P_{T}$ and $M_{\text {all }}$ distributions. The region of most interest for a search for new physics signals is thus the region of greatest deviation, quantified by the the smallest $p$-value, $p_{\text {min }}$.

Such a method is able to find narrow resonances and single outstanding events as well as signals spread over large regions of phase space in distributions of any shape.

The identification of regions of most interest carried out by the search algorithm is illustrated in figure 7.1. Exemplarily shown are the already discussed $M_{\text {all }}$ distributions of the $e-j, j-\nu$ and $j$-j event classes (cf. figure 6.2), now using histograms with a bin size of $5 \mathrm{GeV}$. The shaded areas specify the regions of most interest chosen by the search algorithm. 
Regions of different type, size and position in the distributions are identified by the algorithm as those with the smallest $p$-value for the three event classes. While deficit regions at moderate invariant masses consisting of multiple events are selected by the algorithm in the distributions of the $e-j$ and $j-\nu$ classes, a single outstanding event with an invariant mass of $244 \mathrm{GeV}$ and a small SM expectation of $0.04 \pm 0.02$ events in the region at $240<M_{\text {all }}<260 \mathrm{GeV}$ is found in the $j$ - $j$ event class. The according $p$-value amounts to 0.036. For the $e-j$ event class $139.5 \pm 21.4$ events are predicted by the SM in the selected deficit region at $185<M_{\text {all }}<210 \mathrm{GeV}$ while only 111 data events are measured, resulting in a $p$-value of 0.120 .

Maybe contrary to first expectations the smallest probability of those three distributions discussed is found in the deficit region of the $j-\nu$ event class. A $p$-value of 0.028 is calculated for the region at $155<M_{\text {all }}<180 \mathrm{GeV}$, where 83 data events are measured compared to a SM prediction of $116.8 \pm 14.4$ events.

However, these $p$-values obtained do not take into account the fact that all possible choices of invariant mass regions in the histograms are considered by the search algorithm. This may significantly change the probabilities of the observations and thus has a strong impact on the inference one can make.

The possibility that a fluctuation with a value $p_{\min }$ occurs somewhere in the distributions is considered by the calculation of the significance of the particular deviations observed.

\section{Significance Per Event Class}

In order to determine the significance of the deviation found by the search algorithm by selecting the region with the smallest $p$-value out of all possible choices of regions in the distribution of a particular event class, the probability $\hat{P}$ is calculated to observe a deviation with a $p$-value smaller than $p_{\min }$ in any region of the distribution.

For each investigated distribution many independent hypothetical data histograms $H_{h y p}$ are generated by filling each bin with an event number diced according to the pdf's of the corresponding SM expectations, which are again derived from the convolution of Poisson and Gaussian pdf's. For each of those hypothetical data histograms the algorithm is run to find the region of greatest deviation and the corresponding $p_{\min }^{S M}$-value is calculated.

The probability $\hat{P}$ is then defined as the fraction of hypothetical data histograms with a value of $p_{\min }^{S M}$ equal or smaller than the $p_{m i n}$-value obtained from the real data:

$$
\hat{P}=\frac{\text { number of } H_{\text {hyp }} \text { with } \quad p_{\text {min }}^{S M} \leq p_{\text {min }}^{\text {data }}}{\text { total number of } H_{\text {hyp }}} .
$$

The obtained values of $\hat{P}$ are a measure of the statistical significance of the deviations observed in the data distributions. Following the laws of Poisson statistics the $\hat{P}$ values of event classes containing no data event and a SM expectation $\lesssim 1$ are 1 .

Since the event classes studied are exclusive, the values of $\hat{P}$ can be used to compare the results of the different event classes. Consequently that event class of most interest for a search is the one with the smallest $\hat{P}$ value. 
Coming back to the exemplarily investigation of the $M_{\text {all }}$ distributions of the $e-j, j-\nu$ and $j j$ event classes the impact of the $\hat{P}$ calculation on the results can be illustrated. While based on the obtained $p$-values the deviation observed in the $j$ - $\nu$ event class suggested to be the most interesting, the according $\hat{P}$ value of 0.34 adjusts its significance to that of the $j-j$ event class, where a similar value of 0.38 is found. In the $e-j$ event class a $\hat{P}$ value of 0.94 is calculated, representing the good overall agreement between the data and SM prediction in the $M_{\text {all }}$ distribution of this event class.

A matter of particular interest in a search for new physics signals is the question of a certain significance found for a deviation, in order to consider that a clear discovery has taken place at a certain confidence level. Therefore, usually a $5 \sigma$ criterion is used such that the signal plus background contribution must be above a $5 \sigma$ fluctuation of the background alone. Studies have shown that a $p_{\min }$-value of $5.7 \cdot 10^{-7}$, which corresponds to a $5 \sigma$ fluctuation, results in values of $-\log _{10} \hat{P}$ between 5 and 6 , depending on the final state $^{1}$. These numbers reveal that the previously discussed $M_{\text {all }}$ distributions of all three event classes are well in agreement with the SM expectation.

Nevertheless, among the multitude of studied event classes in this analysis there is some chance that small $\hat{P}$ values occur. This chance is accounted for by the calculation of a global significance based on so-called HERA Monte Carlo experiments, introduced in the following section.

\subsection{Monte Carlo Experiments and Global Significance}

The level of overall agreement between the data and the SM prediction is determined by taking into account the chance, that small $\hat{P}$ values occur among the multiplicity of studied event classes. This chance can be quantified by calculating the number of socalled HERA Monte Carlo experiments, which would find deviations more significant than the one observed in the $\mathrm{H} 1$ data. This final number can be compared between different experiments, even if they investigate a different number of event classes.

\section{HERA Monte Carlo Experiments}

The formalism of HERA Monte Carlo experiments allows for a comparison between the $\hat{P}$ values obtained from the data with an expectation. A HERA Monte Carlo experiment is defined by a set of hypothetical data histograms, which replace the invariant mass and the sum of transverse momenta distributions of all event classes obtained from the data analysis. As described in section 7.1, these hypothetical data histograms are derived from the SM expectation, each representing a single HERA experiment with an integrated luminosity of $117 \mathrm{pb}^{-1}$.

The complete search algorithm as introduced in section 7.1 is applied on these histograms

\footnotetext{
${ }^{1}$ In order to better cope with small numbers the negative decade logarithm of $\hat{P}$ is typically used in the following discussions.
} 

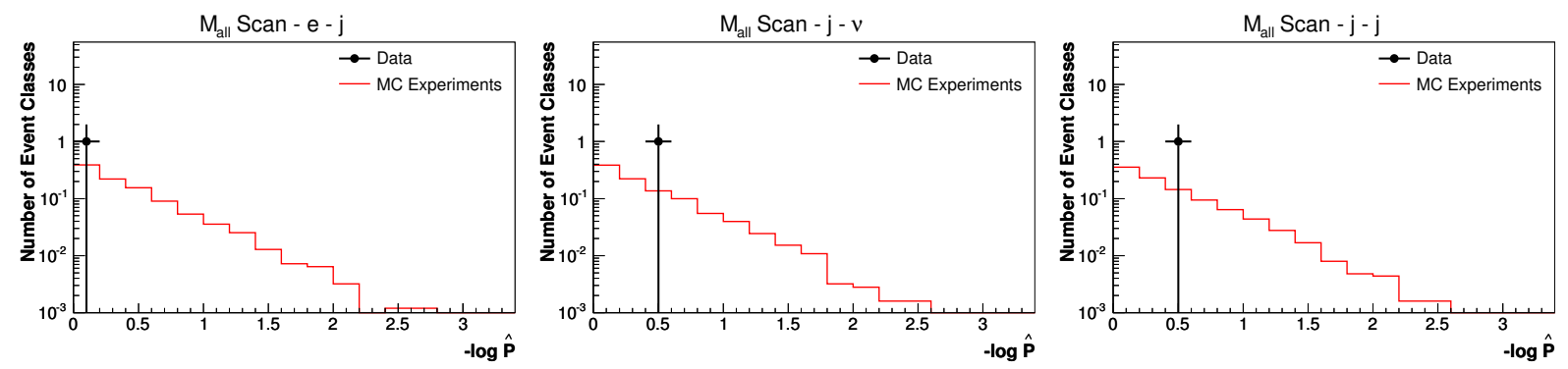

Fig. 7.2: The $-\log _{10} \hat{P}$ values obtained from data for the $e-j, j-\nu$ and $j-j$ event classes and the expected distributions from HERA MC experiments as derived by investigating the invariant mass $M_{\text {all }}$ distributions with the search algorithm.

and thus provides a set of $\hat{P}_{S M}$ values for all event classes, based on the SM expectation only, without involving any data distribution. This procedure is repeated many times. The expectation for the $\hat{P}$ values observed in the data is then given by the distributions of the $\hat{P}_{S M}$ values obtained by multiple HERA Monte Carlo experiments.

Figure 7.2 presents the $\hat{P}_{S M}$ distributions as derived from the investigation of the invariant mass distributions in HERA Monte Carlo experiments together with the $\hat{P}$ values obtained from data for the discussed $e-j, j-\nu$ and $j-j$ event classes. A double-logarithmic scale is chosen in order to focus on the region of small $\hat{P}$ values.

The $\hat{P}_{S M}$ distributions are based on 2500 HERA Monte Carlo experiments and normalised to unity in order to compare with the $\hat{P}$ values observed in the data. Most $-\log _{10} \hat{P}_{S M}$ values range from 0 to 1 , corresponding to HERA Monte Carlo experiments where no significant discrepancy is produced. In the tails of the distributions values between 2 and 3 are reached.

The diagrams corroborate that the $\hat{P}$ values obtained from the data are well in agreement with the SM expectation for these three exemplarily discussed event classes. The results for all event classes are in detail introduced in section 7.4.

\section{Global Significance}

Based on the formalism of HERA Monte Carlo experiments a probability can be calculated, which quantifies the overall agreement between the HERA-I data at high transverse momentum, manifested in the event classes investigated in this analysis, and the Standard Model expectation.

The probability $P_{X}^{n}$ to observe in the data a value of $-\log _{10} \hat{P}$ greater than $X$ in at least $n$ event classes out of the total number of studied event classes is given by the fraction of HERA Monte Carlo experiments having at least $n$ event classes with $-\log _{10} \hat{P}_{S M}>X$. This probability quantifies the global significance of the analysis and defines a criterion to consider that a clear discovery has taken place at a certain confidence level.

The $P_{X}^{n}$ values obtained from the investigation of 2500 HERA Monte Carlo experiments are summarised in table 7.2. Since very similar $P_{X}^{n}$ values are found for the $M_{\text {all }}$ and 


\begin{tabular}{|c||c|c|c|c|c|c|c|c|c|}
\hline$n$ & $P_{1}^{n}$ & $P_{1.5}$ & $P_{2}$ & $P_{2.5}$ & $P_{3}$ & $P_{3.5}$ & $P_{4}$ & $P_{4.5}$ & $P_{5}$ \\
\hline 1 & $95 \%$ & $65 \%$ & $28 \%$ & $9 \%$ & $3 \%$ & $0.9 \%$ & $0.2 \%$ & $0.1 \%$ & $<0.05 \%$ \\
2 & $79 \%$ & $28 \%$ & $4 \%$ & $0.6 \%$ & $0.1 \%$ & $<0.05 \%$ & - & - & - \\
3 & $53 \%$ & $8 \%$ & $0.4 \%$ & $0.05 \%$ & $<0.05 \%$ & - & - & - & - \\
\hline
\end{tabular}

Tab. 7.2: The likeliness $P_{X}^{n}$ to find at least $n$ event classes with a $-\log _{10} \hat{P}$ value greater than $X$. The values obtained from the distributions of $M_{\text {all }}$ or $\sum P_{T}$ are equal.

$\sum P_{T}$ distributions averaged values are presented. At least one deviation with a $-\log _{10} \hat{P}$ value for instance greater than 1, 2 or 3 would be produced by about $95 \%, 28 \%$ or $3 \%$ of HERA MC experiments, respectively.

A $P_{X}^{n}$ value smaller than 0.0005 is expected to be found either in the investigation of the $M_{\text {all }}$ or the $\sum P_{T}$ distributions in a combination of 1,2 or 3 event classes in around $0.1 \%$ of the HERA MC experiments. Therefore a $P_{X}^{n}$ value smaller than 0.0005 is defined as a significant deviation.

\subsection{Sensitivity to New Physics Signals}

A set of pseudo data samples has been used to test the sensitivity of the presented analysis procedure on some dedicated signals of new physics.

In analogy to the formalism of HERA Monte Carlo experiments described in section 7.2 the sum of transverse momenta and invariant mass distributions obtained from data are replaced by hypothetical data histograms. But while the histograms of the HERA Monte Carlo experiments are based on the the SM expectation alone, now the prediction of a specific model for new physics is added to the SM expectation and the resulting distributions are used to generate hypothetical data histograms.

The complete algorithm is run on those BSM Monte Carlo experiments, providing a set of $\hat{P}_{B S M}$ values for all event classes. This procedure is repeated many times and the mean values of $-\log _{10} \hat{P}_{B S M}$ are derived in all of them. Based on these values those event classes sensitive to the signatures of the various new physics models are figured out and the overall sensitivity of this analysis is tested depending on selected model parameters. The reason for choosing the mean of the $-\log _{10} \hat{P}$ values instead of the averaged $\hat{P}$ values themselves as the measure of sensitivity of this analysis may be illustrated by the following example. Similar mean values are obtained from both the pairs of $\hat{P}$ values 0.1 and 1 as well as $1 \cdot 10^{-4}$ and 1 , underestimating the observation of a significant deviation in the latter case.

\section{Anomalous Top Quark Production}

The anomalous production of top quarks in a flavour changing neutral current process has been chosen as one model of new physics in order to test the sensitivity of the analysis [67]. 

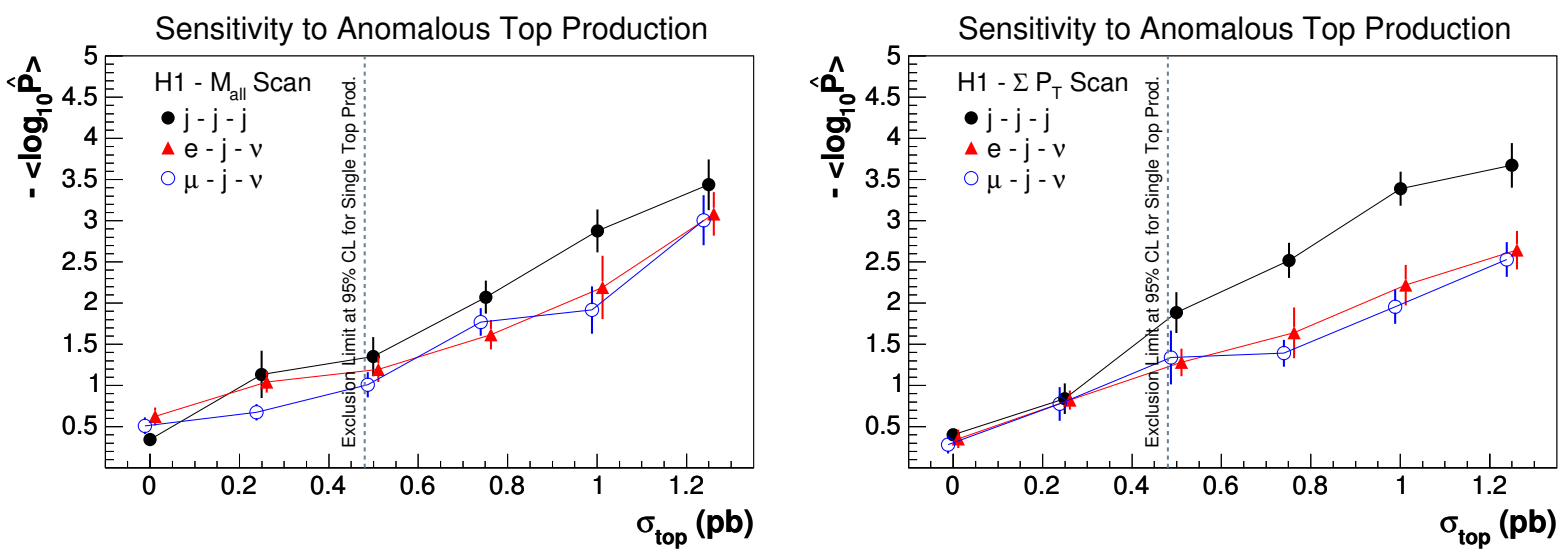

Fig. 7.3: The mean values of $-\log _{10} \hat{P}$ as derived from BSM Monte Carlo experiments including a top signal with a cross section $\sigma_{\text {top }}$. Shown are the results of the $j-j-j, e-j-\nu$ and $\mu-j-\nu$ event classes corresponding to the typical top decay channels.

Only typical top decay channels have been considered.

The $-\left\langle\log _{10} \hat{P}\right\rangle$ values as derived from Monte Carlo experiments including a top signal are displayed in figure 7.3 as a function of the top production cross section. Shown are the results obtained from the investigation of the $M_{\text {all }}$ and $\sum P_{T}$ distributions of the $j-j-j$, $e-j-\nu$ and $\mu-j-\nu$ event classes.

Whereas values around 0.5 are found for $-\left\langle\log _{10} \hat{P}\right\rangle$ if no signal is present, these values increase with the top production cross section for all event classes. The highest value close to 4 is reached for a cross section $\sigma_{\text {top }}$ of $1.25 \mathrm{pb}$ in the scan of the $\sum P_{T}$ distributions for the $j-j-j$ event class. The upper diagrams of figure 7.6 display exemplarily the regions of greatest deviation selected by the search algorithm in the investigation of the $M_{\text {all }}$ and $\sum P_{T}$ distributions of the $j-j-j$ event class in case of adding a top signal with a cross section of $0.75 \mathrm{pb}$ to the $\mathrm{SM}$ expectation.

Comparing the $-\left\langle\log _{10} \hat{P}\right\rangle$ values derived for all event classes by investigating both the $M_{\text {all }}$ and $\sum P_{T}$ distributions, the results obtained from the scan of the $\sum P_{T}$ distributions of the $j$ - $j$ - $j$ event class are found to be most sensitive. There a $-\left\langle\log _{10} \hat{P}\right\rangle$ value of around 2 is observed for a cross section $\sigma_{\text {top }}$ of $0.5 \mathrm{pb}$. This value can be compared to the upper limit of $0.48 \mathrm{pb}$ on the anomalous top production cross section at $95 \%$ confidence level, which has been determined by the $\mathrm{H} 1$ collaboration using the hadronic top decay channel only [67].

\section{Leptoquark Production}

The production of leptoquarks is chosen as another model in order to test the sensitivity of the present analysis. Based on the phenomenological approach proposed by Buchmüller, Rückl and Wyler [68] $S_{1 / 2, L}$ and $V_{0, L}$ type leptoquarks have been considered, which would mainly manifest themselves in neutral and charged current like event classes. A $\lambda$ coupling 

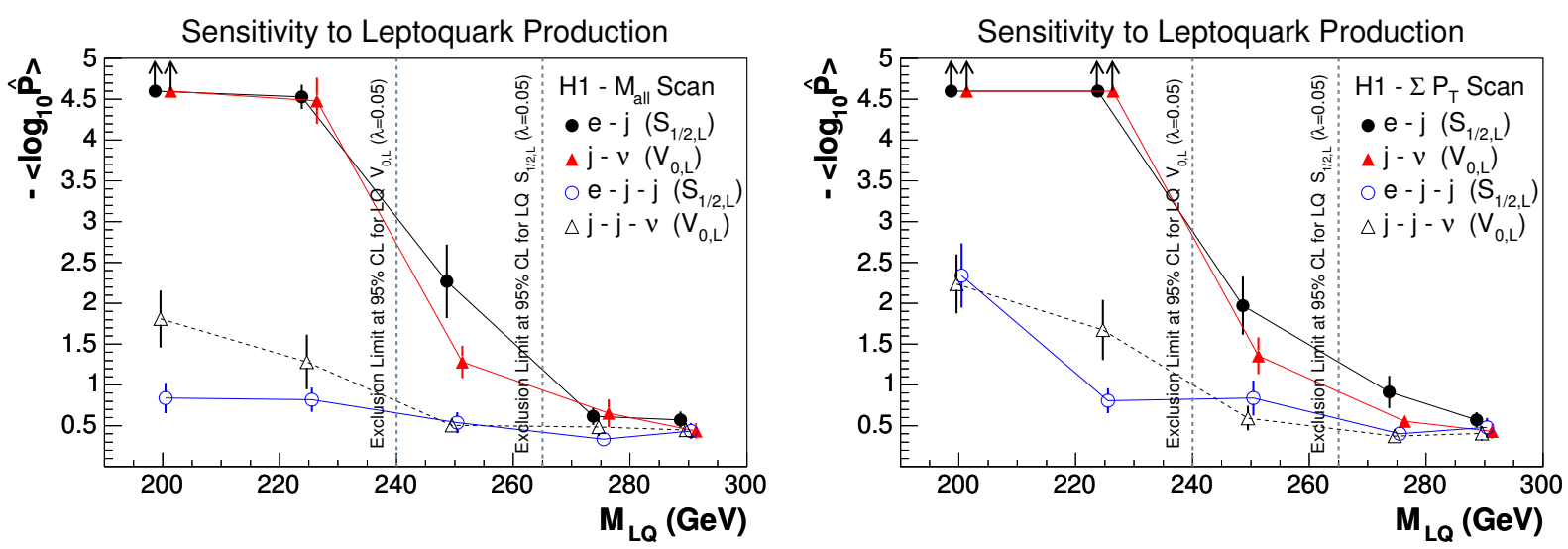

Fig. 7.4: The mean values of $-\log _{10} \hat{P}$ as derived from BSM Monte Carlo experiments including a leptoquark signal with a mass $M_{L Q} . S_{1 / 2, L}$ and $V_{0, L}$ type leptoquarks have been considered, which are expected to manifest themselves in the $e-j$ and $e-j-j$ as well as in the $j-\nu$ and $j-j-\nu$ event classes, respectively.

of 0.05 is assumed and the sensitivity of the analysis is tested depending on the mass of the leptoquarks.

The $-\left\langle\log _{10} \hat{P}\right\rangle$ values obtained from the scan of the invariant mass and sum of transverse momenta distributions including a leptoquark signal are shown in figure 7.4. In order to conserve computing time $\hat{P}$ values smaller than $10^{-5}$ are no longer accurately calculated and must be treated as upper limit.

The highest sensitivity on the production of $S_{1 / 2, L}$ and $V_{0, L}$ type leptoquarks is achieved in the $e-j$ and $j-\nu$ event class, respectively, reaching $-\left\langle\log _{10} \hat{P}\right\rangle$ values above 4.5 for leptoquark masses below $225 \mathrm{GeV}$. In the same mass region values for $-\left\langle\log _{10} \hat{P}\right\rangle$ between 1 and 2.5 are derived for the $e-j-j$ and $j-j-\nu$ event classes. In particular for these classes the investigation of the $\sum P_{T}$ distributions exhibits a slightly higher sensitivity compared to the results obtained from the $M_{\text {all }}$ scan.

Some examples of regions selected by the search algorithm are presented in the middle part of figure 7.6. Shown are the $M_{\text {all }}$ and $\sum P_{T}$ distributions of the $e-j$ and $j-\nu$ event class including the signal arising from the decay of a $S_{1 / 2, L}$ and $V_{0, L}$ type leptoquark with a mass of $225 \mathrm{GeV}$, respectively.

The study corroborates a sensitivity of this analysis on the production of both types of leptoquarks up to masses between 240 and $250 \mathrm{GeV}$. These values can be compared to the $95 \%$ confidence level limits at $265 \mathrm{GeV}$ for the production of $S_{1 / 2, L}$ and $240 \mathrm{GeV}$ for $V_{0, L}$ type leptoquarks, determined by dedicated analyses [69, 70].

\section{Stop Quark Production}

Finally, the resonant production of scalar top quarks $\tilde{t}$ in $R$-parity violating supersymmetry is consulted to test the sensitivity of the presented analysis procedure [52, 71]. Both 

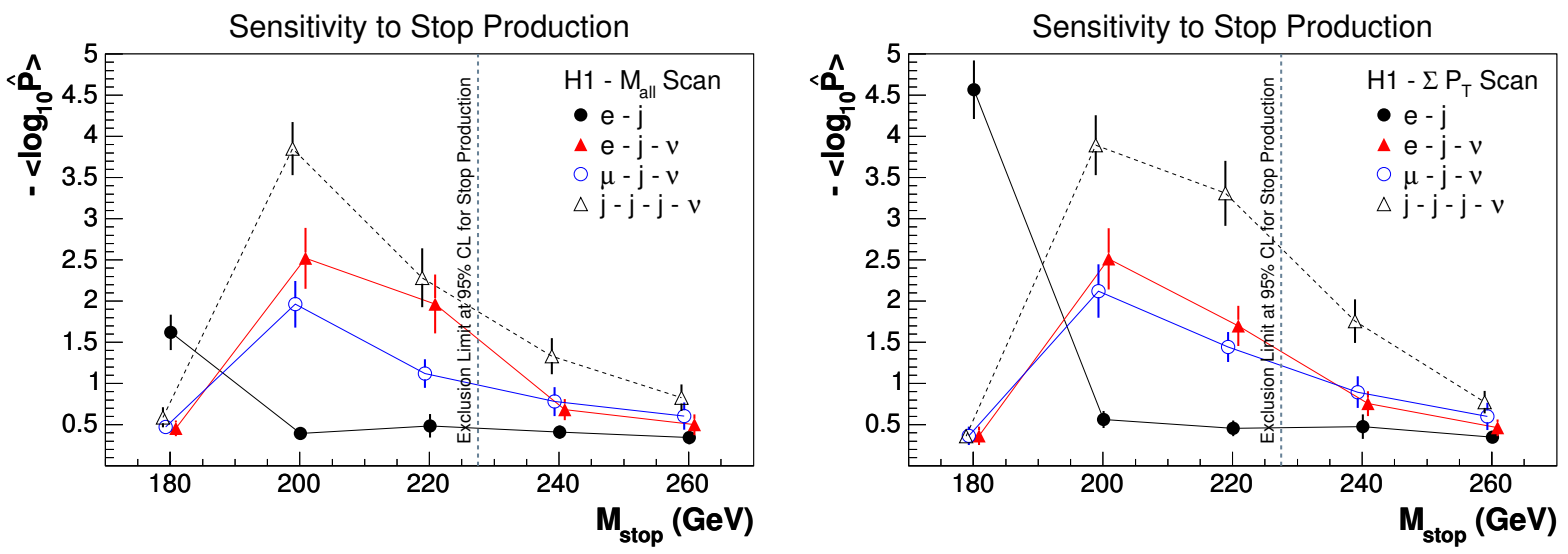

Fig. 7.5: The mean values of $-\log _{10} \hat{P}$ as derived from BSM Monte Carlo experiments including a stop signal with a mass $M_{\text {stop }}$. Shown are the results of the $e-j-\nu, \mu-j-\nu$ and $j-j-j-\nu$ event classes as well as for the $e-j$ event class, which are expected to be sensitive on the bosonic and direct decay of the stop quark, respectively.

the bosonic stop decay $\tilde{t} \rightarrow \tilde{b} W$ with the subsequent $R$-parity violating decay $\tilde{b} \rightarrow d \bar{\nu}_{e}$ and the direct $R$-parity violating decay $\tilde{t} \rightarrow e q$ are considered. Depending on the stop mass the sensitivity of the analysis on the production of scalar top quarks is tested with the according coupling $\lambda_{131}^{\prime}$ set to 0.1 and a sbottom mass of $100 \mathrm{GeV}$.

The $-\left\langle\log _{10} \hat{P}\right\rangle$ values as derived from this study are presented in figure 7.5 for both the scan of the sum of transverse momenta and invariant mass distributions. Based on the leptonic and hadronic decay of the $W$ boson the results obtained from the investigation of the $e-j-\nu, \mu-j-\nu$ and $j-j-j-\nu$ event classes are shown. The direct $R$-parity violating decay $\tilde{t} \rightarrow e q$ is expected to manifest itself in the distributions of the $e-j$ event class.

As expected, the sensitivity on stop quarks decaying bosonically is decreasing with the stop mass. The highest $-\left\langle\log _{10} \hat{P}\right\rangle$ values between 3 and 4 are found for stop masses of 220 and $200 \mathrm{GeV}$ in the $j-j-j-\nu$ event class by investigating the $\sum P_{T}$ distributions. In the same mass region values of $-\left\langle\log _{10} \hat{P}\right\rangle$ ranging from 1.5 to 2.5 are derived for the $e-j-\nu$ and $\mu-j-\nu$ event classes.

For the lowest considered stop mass of $180 \mathrm{GeV}$ no signal is observed anymore in those event classes expected to be sensitive on the bosonic decay, while $-\left\langle\log _{10} \hat{P}\right\rangle$ values of 1.6 and 4.5 are derived for the $e-j$ event class in the scan of the according $M_{\text {all }}$ and $\sum P_{T}$ distributions, respectively. An example of regions of greatest deviation selected by the search algorithm for this scenario is presented in the lower diagrams of figure 7.6.

For higher stop masses only $-\left\langle\log _{10} \hat{P}\right\rangle$ values around 0.5 are found in the investigation of the $e-j$ event class.

This behaviour can be explained by the characteristics of the branching ratios of the stop decay depending on the chosen model parameters. For a stop mass in the order of the sum of the masses of the sbottom quark and the $W$ boson the direct $R$-parity violating decay $\tilde{t} \rightarrow e q$ becomes dominant. Since the according branching ratio decreases steeply 

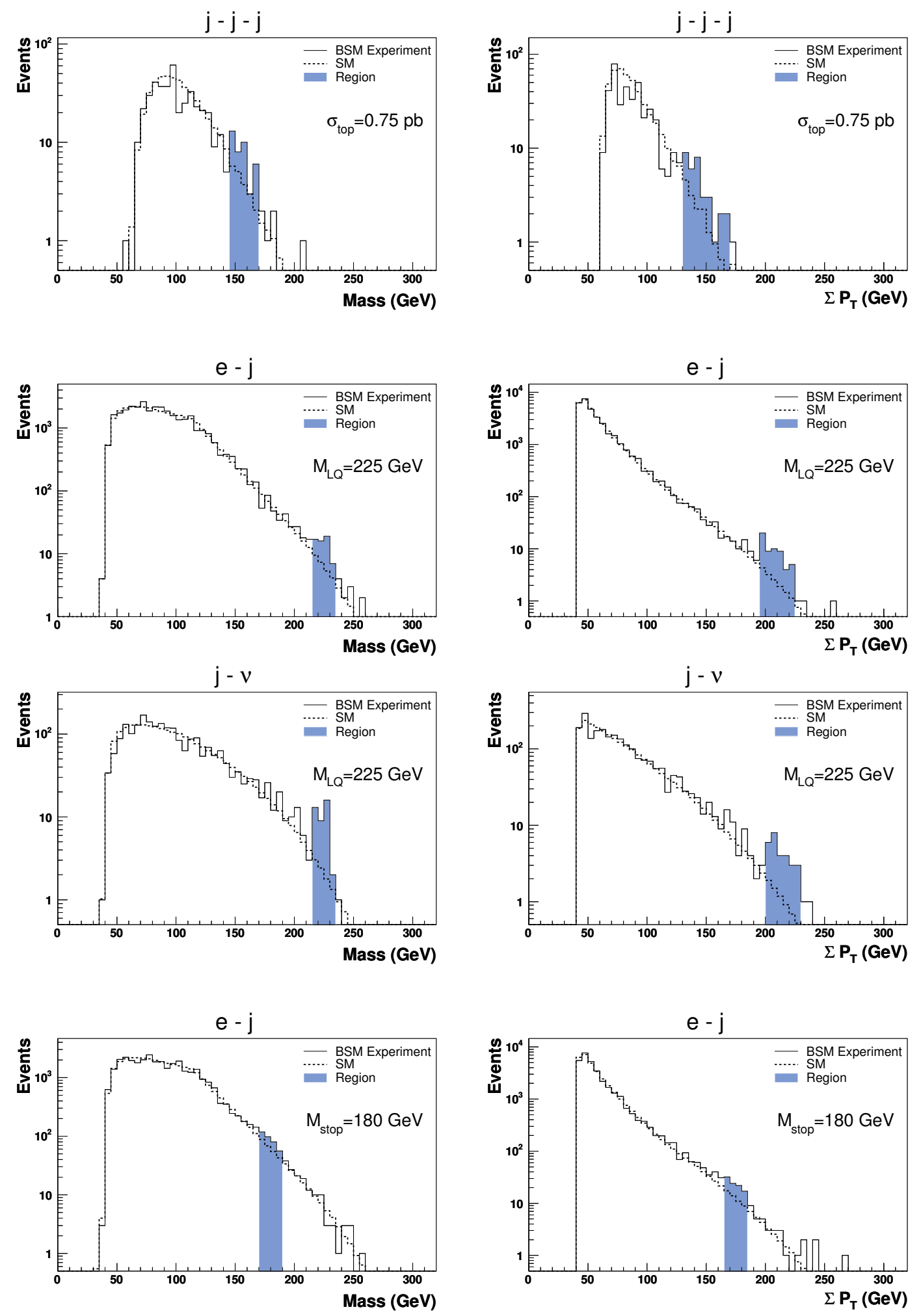

Fig. 7.6: The $M_{\text {all }}$ and $\sum P_{T}$ distributions obtained from BSM Monte Carlo experiments including various signals of new physics and the according SM distributions. The shaded areas show the regions of greatest deviation chosen by the search algorithm. 
with the stop mass, the direct decay is accessible only for a stop mass of $180 \mathrm{GeV}$, while for higher masses a sensitivity on the bosonic decay is observed.

Consequently, the results obtained reveal a sensitivity of the presented analysis on the production of stop quarks up to masses of $220 \mathrm{GeV}$. Again a slightly higher sensitivity is observed by the investigation of the sum of transverse momenta distributions. The upper limit established at 95\% confidence level as derived by a dedicated analysis [52] excludes stop quark production in $R$-parity violating supersymmetry up to masses of $227.5 \mathrm{GeV}$, supporting the sensitivity observed in this analysis.

The results derived from the study of these three pseudo data samples demonstrate that the presented general search analysis has a rather good sensitivity on signals of new physics in the range, where exclusion limits have been set by dedicated searches. It has been shown that both the sum of transverse momenta and the invariant mass of all objects in the event classes are well suited in order to search for new physics signals at large energies.

\subsection{Search Results}

In order to systematically search the HERA-I data for new physics signals all event classes are passed through the statistical analysis. The regions of greatest deviation have been determined by the search algorithm in both the sum of transverse momenta and invariant mass distributions and their significance has been calculated. Therewith the presented analysis allows for the first time a quantification of the overall agreement between the HERA-I data at high transverse momentum and the Standard Model prediction.

The final $\hat{P}$ values determined by the search algorithm from the investigation of the $\sum P_{T}$ and $M_{\text {all }}$ distributions are summarised in table 7.3 for all event classes containing data events. The $\hat{P}$ values of event classes with no data event and a SM expectation $\lesssim 1$ are 1 . Since the SM expectation of the $j-j-j-j$ and $e-j-j-j-j$ event classes may suffer from rare SM processes not considered in this analysis (cf. section 6), their results are separated from the results obtained from the other classes and subject to a dedicated discussion.

For all event classes the according regions of greatest deviation selected by the search algorithm are presented in figure 7.9 as derived from the $M_{\text {all }}$ scan and in figure 7.10 for the $\sum P_{T}$ scan.

The most significant deviation of the analysis is found in the $\mu-j-\nu$ event class. $\hat{P}$ values of $9.7 \cdot 10^{-3}$ and $1.0 \cdot 10^{-3}$ are found in the investigation of the $M_{\text {all }}$ and $\sum P_{T}$ distributions, respectively. The selected mass region contains 3 data events compared to an expectation of $0.19 \pm 0.05$ events. In the chosen $\sum P_{T}$ region 3 data events are found while only $0.07 \pm 0.03$ events are predicted by the SM. This event topology was already studied in [37] and an equivalent discrepancy has been observed.

A $\hat{P}$ value of 0.019 is found in the $e$-e event class in a region at high transverse momenta between $100 \mathrm{GeV}$ and $130 \mathrm{GeV}$, where 3 events are measured compared to an expectation 


\begin{tabular}{|c||c|r|rrr|c|}
\hline \multicolumn{1}{|c||}{} & \multicolumn{7}{|c|}{$\sum P_{T}$-Scan } \\
\hline \hline event class & \multicolumn{1}{|c|}{$\hat{P}$} & $N_{\text {obs }}$ & $N_{S M}$ & \pm & $\delta N_{S M}$ & \multicolumn{1}{c|}{$p$} \\
\hline$j-j$ & 0.12 & 1 & 0.013 & \pm & 0.006 & 0.013 \\
$e-j$ & 0.021 & 12 & 31.2 & \pm & 5.1 & 0.003 \\
$\mu-j$ & 0.29 & 3 & 0.70 & \pm & 0.23 & 0.040 \\
$j-\nu$ & 0.22 & 20 & 36.7 & \pm & 6.2 & 0.023 \\
$e-\nu$ & 0.77 & 0 & 2.1 & \pm & 0.8 & 0.17 \\
$e-e$ & 0.019 & 3 & 0.18 & \pm & 0.08 & 0.001 \\
$e-\mu$ & 0.56 & 0 & 2.6 & \pm & 0.5 & 0.080 \\
$\mu-\mu$ & 0.036 & 2 & 0.11 & \pm & 0.03 & 0.006 \\
$j-\gamma$ & 0.77 & 0 & 2.5 & \pm & 1.0 & 0.13 \\
$e-\gamma$ & 0.64 & 8 & 15.7 & \pm & 1.9 & 0.040 \\
$\gamma-\gamma$ & 0.31 & 1 & 0.11 & \pm & 0.09 & 0.12 \\
$j-j-j$ & 0.58 & 14 & 7.8 & \pm & 2.5 & 0.077 \\
$e-j-j$ & 0.085 & 9 & 23.9 & \pm & 4.4 & 0.007 \\
$j-j-\nu$ & 0.51 & 5 & 1.74 & \pm & 0.45 & 0.040 \\
$e-j-\nu$ & 0.16 & 2 & 0.28 & \pm & 0.06 & 0.034 \\
$\mu-j-\nu$ & $1.0 \cdot 10^{-3}$ & 3 & 0.068 & \pm & 0.029 & $7.5 \cdot 10^{-5}$ \\
$j-j-\gamma$ & 0.36 & 1 & 0.15 & \pm & 0.10 & 0.15 \\
$e-j-\gamma$ & 0.39 & 1 & 5.6 & \pm & 1.4 & 0.045 \\
$e-j-j-j$ & 0.87 & 1 & 0.18 & \pm & 0.06 & 0.17 \\
$j-j-j-\nu$ & 0.20 & 2 & 0.31 & \pm & 0.14 & 0.044 \\
\hline \hline$j-j-j-j$ & 0.048 & 2 & 0.115 & \pm & 0.070 & 0.008 \\
$e-j-j-j-j$ & $1.0 \cdot 10^{-3}$ & 1 & $3.6 \cdot 10^{-4}$ & \pm & $2.7 \cdot 10^{-4}$ & $4.1 \cdot 10^{-4}$ \\
\hline
\end{tabular}

\begin{tabular}{|c||c|r|rrr|c|}
\hline \multicolumn{1}{|c||}{} & \multicolumn{7}{|c|}{$M_{\text {all }}$-Scan } \\
\hline \hline event class & $\hat{P}$ & $N_{\text {obs }}$ & $N_{S M}$ & \pm & $\delta N_{S M}$ & \multicolumn{1}{c|}{$p$} \\
\hline$j-j$ & 0.38 & 1 & 0.035 & \pm & 0.017 & 0.036 \\
$\mu-j$ & 0.94 & 111 & 139 & \pm & 21 & 0.12 \\
$j-\nu$ & 0.67 & 3 & 1.07 & \pm & 0.25 & 0.098 \\
$e-\nu$ & 0.34 & 83 & 116 & \pm & 14 & 0.028 \\
$e-e$ & 0.94 & 5 & 10.6 & \pm & 4.4 & 0.17 \\
$e-\mu$ & 0.32 & 3 & 0.56 & \pm & 0.17 & 0.023 \\
$\mu-\mu$ & 0.21 & 4 & 0.93 & \pm & 0.12 & 0.016 \\
$j-\gamma$ & 0.069 & 2 & 0.14 & \pm & 0.04 & 0.010 \\
$e-\gamma$ & 0.52 & 3 & 10.8 & \pm & 3.7 & 0.052 \\
$\gamma-\gamma$ & 0.38 & 9 & 19.2 & \pm & 2.0 & 0.014 \\
$j-j-j$ & 0.47 & 1 & 0.16 & \pm & 0.09 & 0.15 \\
$e-j-j$ & 0.41 & 12 & 5.9 & \pm & 2.0 & 0.050 \\
$j-j-\nu$ & 0.69 & 39 & 59.6 & \pm & 10.7 & 0.058 \\
$e-j-\nu$ & 0.62 & 5 & 1.79 & \pm & 0.41 & 0.043 \\
$\mu-j-\nu$ & 0.090 & 2 & 0.19 & \pm & 0.05 & 0.016 \\
$j-j-\gamma$ & $0.70^{-3}$ & 3 & 0.19 & \pm & 0.05 & 0.001 \\
$e-j-\gamma$ & 0.27 & 1 & 0.074 & \pm & 0.048 & 0.076 \\
$e-j-j-j$ & 0.98 & 1 & 5.7 & \pm & 1.6 & 0.05 \\
$j-j-j-\nu$ & 0.33 & 1 & 0.084 & \pm & 0.045 & 0.23 \\
\hline \hline$j-j-j-j$ & 0.091 & 4 & 0.760 & \pm & 0.355 & 0.083 \\
$e-j-j-j-j$ & $3.1 \cdot 10^{-4}$ & 1 & $9.0 \cdot 10^{-5}$ & \pm & $5.8 \cdot 10^{-5}$ & $9.7 \cdot 10^{-5}$ \\
\hline
\end{tabular}

Tab. 7.3: The $\hat{P}$ values, the number of data events $N_{\text {obs }}$ and the SM expectation $N_{S M}$ of the region derived by the search algorithm using the $M_{\text {all }}$ and $\sum P_{T}$ distributions for all event classes. The $p$ value in the selected region is also presented. 
of $0.18 \pm 0.08$ events. The discrepancy observed in the invariant mass distribution is less prominent due to a higher background arising from neutral current DIS events for this observable. The deviations realised in this analysis can be compared to the excess of data events also identified in [38].

A deficit is observed in the sum of transverse momenta distribution of the $e-j$ event class in a region between $180 \mathrm{GeV}$ and $210 \mathrm{GeV}$. Compared to a SM expectation of $31.2 \pm 5.0$ events only 12 data events are measured. The derived $\hat{P}$ value amounts to 0.021 .

Even though rare SM processes not considered in this analysis might contribute to the SM prediction of the $j-j-j-j$ and $e-j-j-j-j$ event classes in the regions of high transverse momenta and invariant masses, both event classes are passed through the statistical analysis revealing some interesting result.

While moderate values of $\hat{P}$ are found for the $j-j-j$-j event class low $\hat{P}$ values of $3.1 \cdot 10^{-4}$ and $1.0 \cdot 10^{-3}$ are found for the $e-j-j-j-j$ event class, corresponding to regions of high invariant masses and sum of transverse momenta, respectively. Both selected regions contain the one measured data event compared to an expectation of $(9.0 \pm 5.8) \cdot 10^{-5}$ events for the $M_{\text {all }}$ and $(3.6 \pm 2.7) \cdot 10^{-4}$ events for the $\sum P_{T}$ distribution.

However, the $\hat{P}$ values depend on a correct reproduction of the SM prediction and should therefore be interpreted with care. But even (or especially) in case of infering the obtained results as indication of a rare SM process contribution, the measurement of an event including an electron and four jets with high transverse momenta is worth the trouble of further investigations in the framework of a dedicated analysis.

The $\hat{P}$ values observed in the data are compared to the distributions of $\hat{P}_{S M}$ obtained from HERA Monte Carlo experiments in figure 7.7. All event classes with a SM expectation greater than 0.01 events are considered except for the $j-j-j-j$ and $e-j-j-j-j$ event classes. Due to the uncertainties in the SM prediction at the highest $M_{\text {all }}$ and $\sum P_{T}$ values, where data events are observed, no reliable $\hat{P}$ values can be calculated for these classes. Consequently, these event classes are not taken into account to determine the overall degree of agreement between the data and the Standard $\mathrm{Model}^{2}$. The distributions of the Monte Carlo experiments are normalised to one HERA experiment. The comparison is presented for the scans of the invariant mass and sum of transverse momenta distributions.

Most $\hat{P}$ values range from 0.01 to 0.99 , corresponding to event classes where no significant discrepancy between data and the SM expectation is observed. These results are in agreement with the expectation obtained from the Monte Carlo experiments.

In order to test the dependence of the obtained results on the a priori defined $P_{T}$ cuts the whole analysis has been repeated with two other transverse momentum conditions on the objects (cf. chapter 6 ). The $P_{T}$ cut was raised to $40 \mathrm{GeV}$ for all objects and lowered to $15 \mathrm{GeV}$ for all objects except one to ensure a high trigger efficiency. The dependence

\footnotetext{
${ }^{2}$ The $\hat{P}$ values for event classes with no data event observed and a small SM expectation are 1 . This remains the case if an additional contribution is added from a possible further rare process not included here. Such classes can thus be considered in the calculation of the global significance.
} 

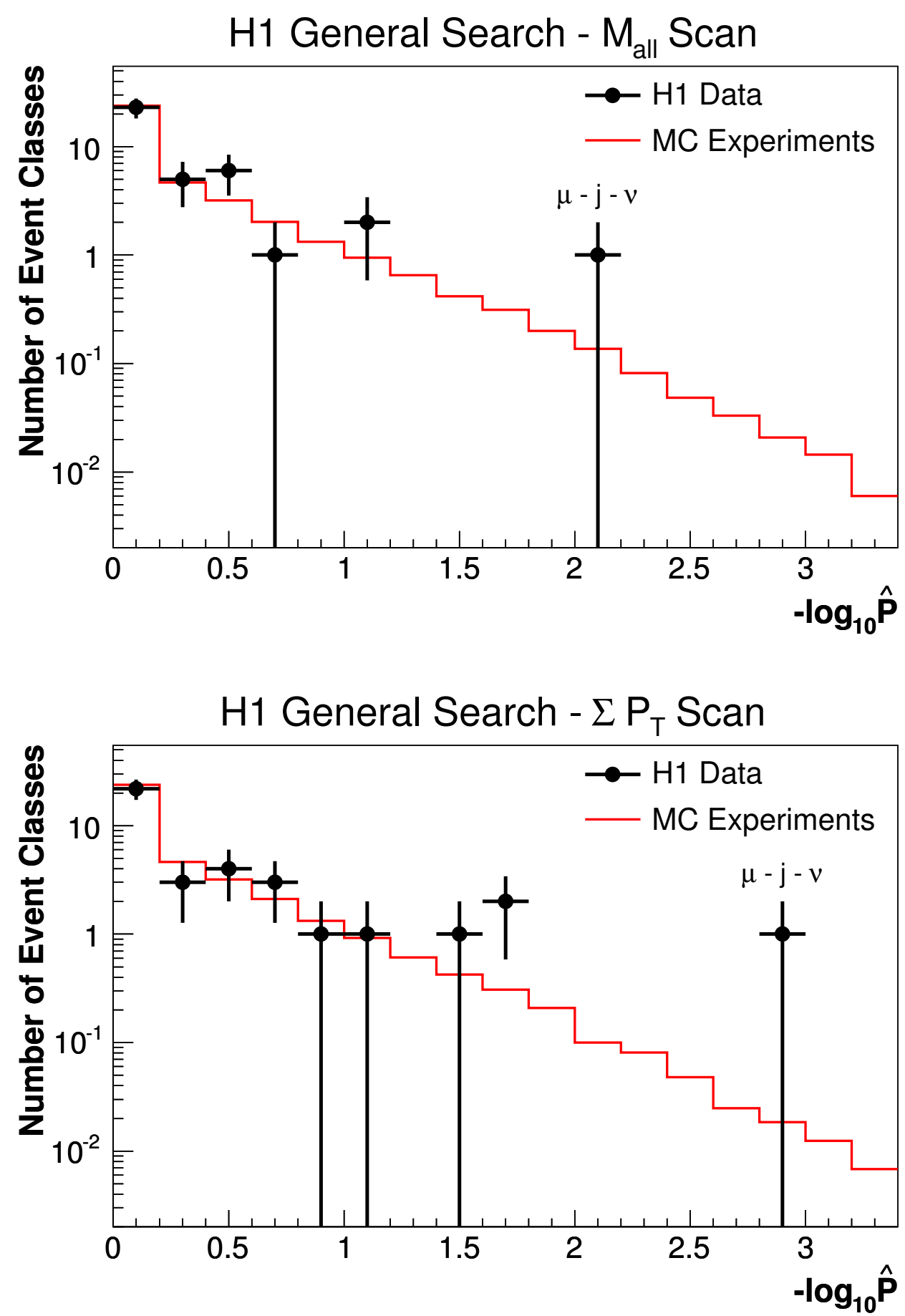

Fig. 7.7: The $-\log _{10} \hat{P}$ values for the data event classes and the expected distributions from $\mathrm{MC}$ experiments as derived by investigating the $M_{\text {all }}$ distributions (upper figure) and $\sum P_{T}$ distributions (lower figure) with the search algorithm. 

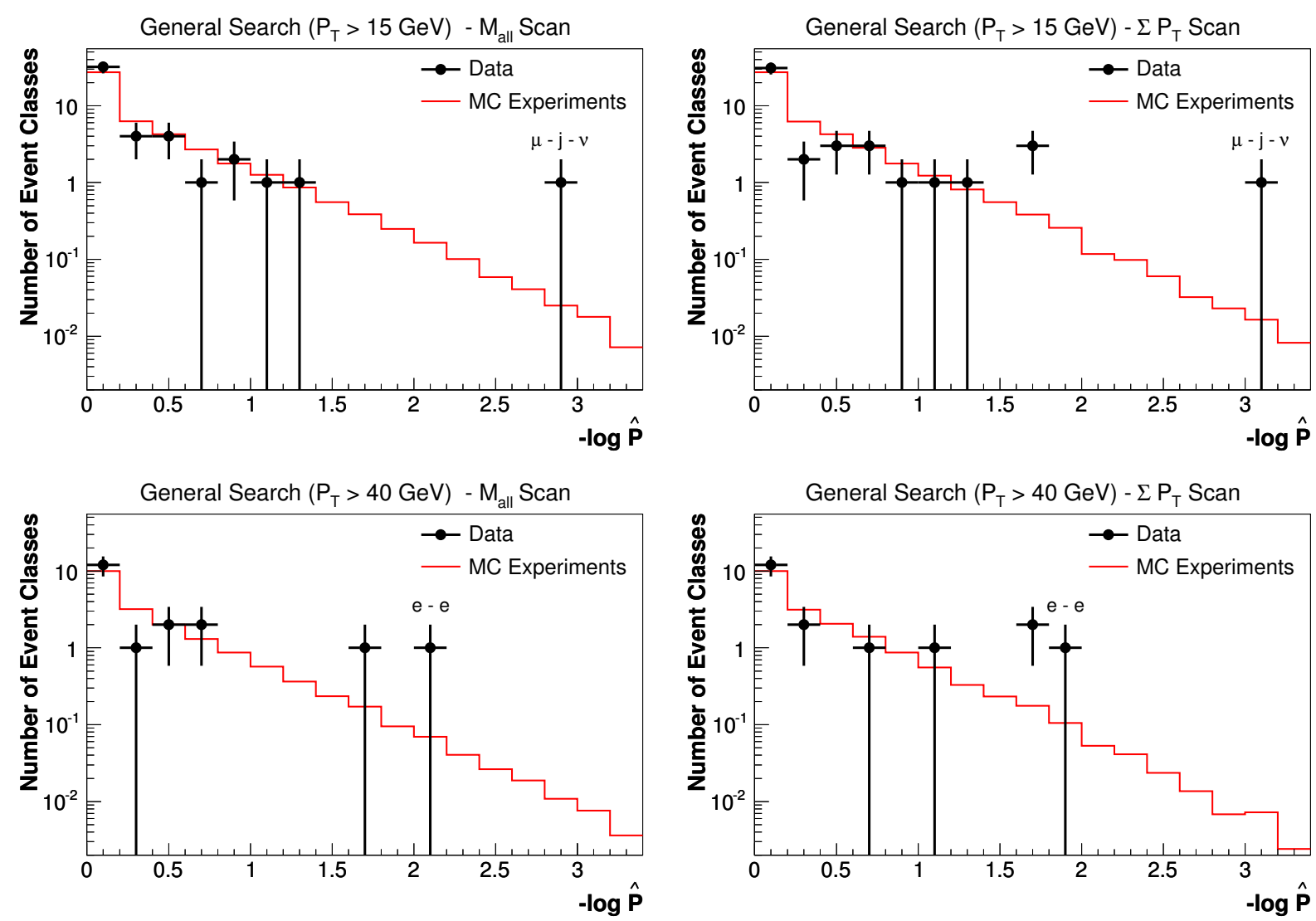

Fig. 7.8: The $-\log _{10} \hat{P}$ values for the data event classes and the expected distributions from MC experiments as derived by investigating the $M_{\text {all }}$ and $\sum P_{T}$ distributions with the search algorithm for the event samples with the object $P_{T}$ cut lowered to $15 \mathrm{GeV}$ (upper figures) and raised to $40 \mathrm{GeV}$ (lower figures).

of the results on combining the $e^{+} p$ and $e^{-} p$ data samples has been verified by applying the full analysis chain on each data sample separately. The according event yields subdivided into the event classes are presented in figure 6.7 for the $e^{+} p$ and $e^{-} p$ data samples and in figure 6.8 for the event samples obtained from the various transverse momentum requirements.

In these four test scenarios a similar agreement between the data results and the SM prediction is observed in the event classes. The $\mu-j-\nu$ event class remains the one with the smallest $\hat{P}$ value in the scenario with a lowered $P_{T}$ cut and in the $e^{+} p$ data sample, and no new discrepancy is observed. For the $P_{T}>40 \mathrm{GeV}$ sample it is mainly the two particle event classes containing jets that are still populated by data events, and the largest deviation is observed in the $e$ - $e$ class with a $\hat{P}$ value of 0.01 .

The $\hat{P}$ values found in the investigation of both event samples with varied transverse momentum criteria are compared to the distributions of $\hat{P}_{S M}$ obtained from HERA Monte Carlo experiments in figure 7.8. For each sample the $\hat{P}$ values derived from the scans of the invariant mass and sum of transverse momenta distributions are shown. 
Finally, the overall agreement between the HERA-I data and the Standard Model is quantified by the probability to find a deviation similar to that observed for the $\mu-j-\nu$ event class in the investigation of the 1994-2000 data sample. According to the determined $\hat{P}$ values this probability is given by the value of $P_{3}^{1}$ for the scan of the sum of transverse momenta distributions and $P_{2}^{1}$ for the scan of the invariant mass distributions. In table 7.2 those probabilities are found to amount to $3 \%$ and $28 \%$, respectively. 


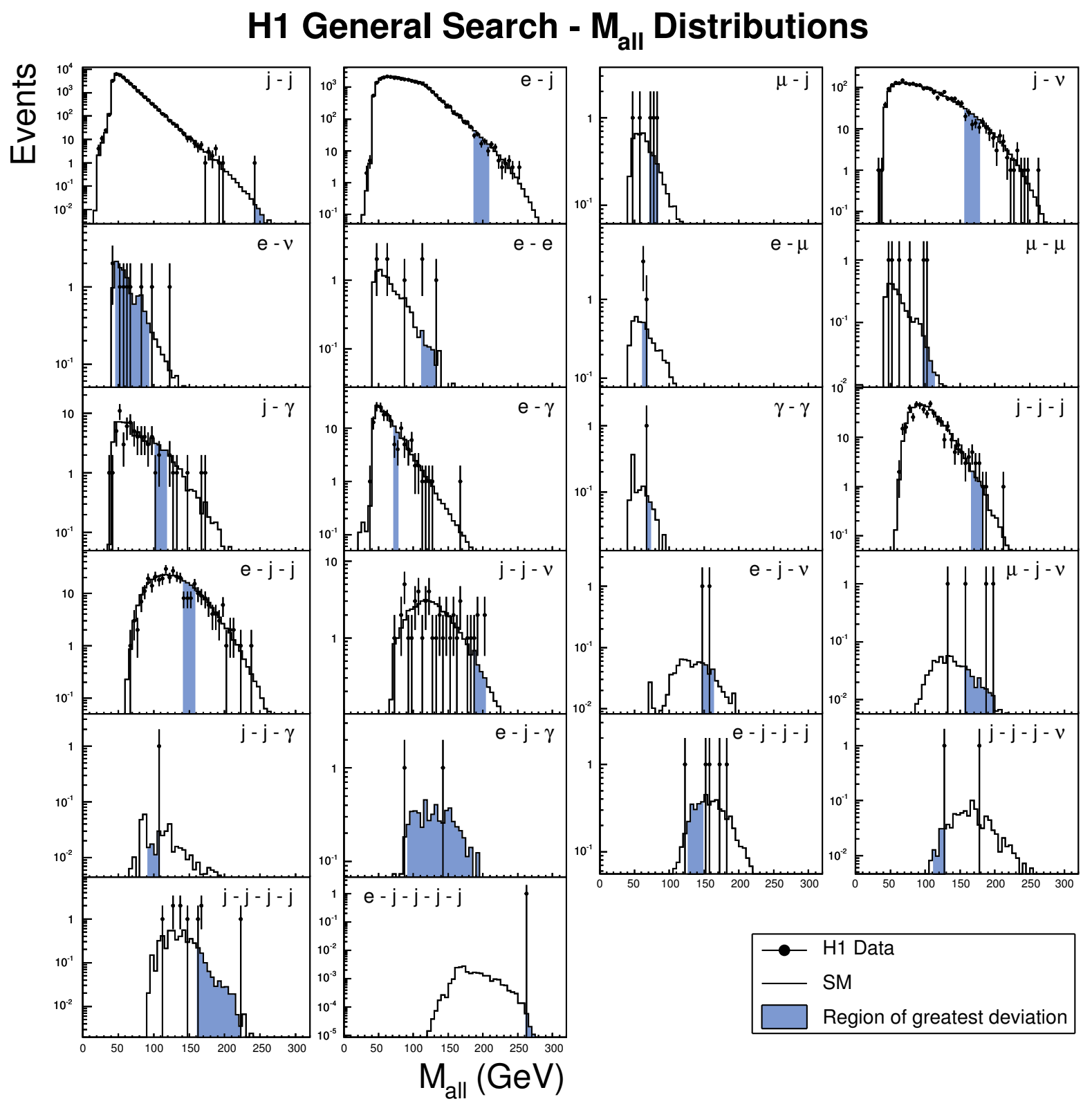

Fig. 7.9: The number of data events and the SM expectation as a function of $M_{\text {all }}$ for event classes with observed data events. The shaded areas show the regions of greatest deviation chosen by the search algorithm. 
H1 General Search $-\Sigma \mathbf{P}_{\mathrm{T}}$ Distributions
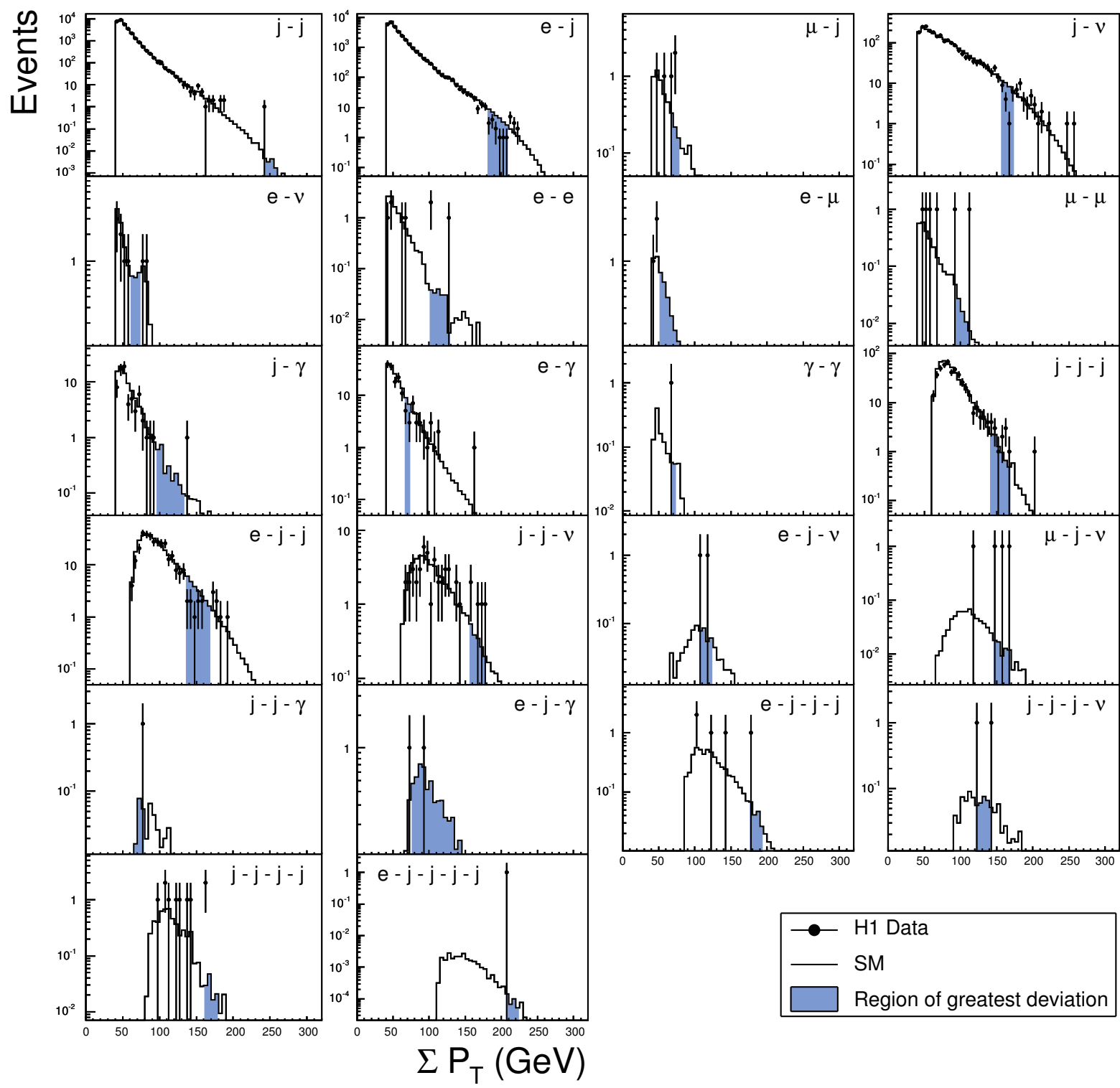

Fig. 7.10: The number of data events and the SM expectation as a function of $\sum P_{T}$ for event classes with observed data events. The shaded areas show the regions of greatest deviation chosen by the search algorithm. 


\section{Summary}

In this thesis a general search for new phenomena in $e p$ scattering at HERA is presented. The data collected with the H1 experiment between the years 1994 and 2000 (HERA-I) are therein systematically explored for deviations from the Standard Model (SM) prediction at high transverse momentum.

The analysis covers phase space regions where the SM prediction is sufficiently precise to detect anomalies and does not rely on assumptions concerning the characteristics of a specific SM extension. This model-independent search might therefore be able to discover unexpected manifestations of new physics. Consequently it would also be ideally suited to effectively scan the data of forthcoming experiments for instance at the Large Hadron Collider LHC, in order to reveal interesting event topologies to be further investigated in dedicated analyses. But though presenting an interesting approach, this kind of analysis requires a profound and precise knowledge of the overall detector performances and might therefore not be able to evolve its entire discovery potential in the early stages of a collider experiment.

For the first time at HERA all event topologies involving isolated electrons, photons, muons, jets and neutrinos are investigated in a coherent analysis. A common phase space defined by a transverse momentum $P_{T}$ above $20 \mathrm{GeV}$ and the polar angle range of $10^{\circ}<\theta<140^{\circ}$ is required for electrons, photons, muons and jets. The neutrino phase space is given by missing transverse momentum $\not_{T}$ above $20 \mathrm{GeV}$ and a longitudinal momentum balance $\sum_{i}\left(E_{i}-P_{z, i}\right)$ below $48 \mathrm{GeV}$.

The identification criteria for each type of particle are based on previous analyses performed on specific final states. Additional requirements have been developed to ensure an unambiguous identification of all particles, whilst keeping high efficiencies.

According to these object definitions all events are divided into exclusive event classes, depending on the number and type of the objects detected in the final state. In total 23 event classes are found to be populated by data, which is in agreement with the SM expectation.

Purities and efficiencies are derived for each event class with a sizeable SM prediction, in order to study the quality of the applied particle identification criteria. Most purities are found to exceed $60 \%$ and reach up to $100 \%$ for the $j-j, e-j, j-\nu, e-\gamma$ and $\mu-\mu$ event classes. The highest efficiencies above $90 \%$ are derived for the $j-j, j-\nu$ and $j-j$-j event classes. The $\mu-\nu$ event class is found to be dominated by unprecisely known low- $P_{T}$ photoproduc- 
tion background in which a low- $P_{T}$ muon track is wrongly reconstructed. It is therefore discarded from further investigations. The background arising from low- $P_{T}$ processes is found to be negligible for all other event classes.

In this analysis events with four high transverse momentum jets are investigated for the first time at HERA. Extensive studies are carried out on low- $P_{T}$ neutral current DIS and photoproduction event samples about the feasibility of an exploration of multi-jet final states, based on MC event generators using the parton shower approach as representation of higher order QCD radiation. A consistent description of the investigated distributions of the data within the quoted SM uncertainties is observed in the test samples.

For all event classes the global yields and the distributions of the scalar sum of transverse momenta $\sum P_{T}$ and the invariant mass $M_{\text {all }}$ of the corresponding high- $P_{T}$ objects are investigated. A good agreement between the data and SM expectation is observed in most event classes.

A discrepancy from the SM prediction is found in the $\mu-j-\nu$ event class, where 4 events are measured compared to an expectation of $0.8 \pm 0.2$ events. A similar deviation was previously reported in [37].

One event is observed in the $e-j-j-j-j$ event class compared to an expectation of $0.026 \pm$ 0.011 events. For this interesting event a $\sum P_{T}$ value of $207 \mathrm{GeV}$ and an invariant mass of $262 \mathrm{GeV}$ is reconstructed. In this kinematic regime the dominant neutral current DIS prediction yields only about $10^{-4}$ events, corresponding to a cross section in the order of $0.001 \mathrm{fb}$. The observed deviation might thus arise from a contribution of rare SM processes, such as the production of $W$ pairs, not considered in this analysis.

In order to systematically search for signals of new physics processes the obtained $M_{\text {all }}$ and $\sum P_{T}$ distributions are investigated for all event classes within the framework of a statistical analysis. Since a better knowledge of rare processes is required to search for deviations from the SM in final states with 4 jets, the results obtained from these event classes are excluded from the interpretation.

A new search algorithm is developed, which locates the regions of possible deviations between the data and SM in the distributions of the researched quantities and calculates the probability of occurrence of such a deviation.

Detailed studies on the sensitivity of the analysis procedure to new physics signals are performed, for which various hypothetical signals have been mixed with the SM expectation. A rather good sensitivity is found in the range where exclusion limits have been set by dedicated analysis.

The most significant deviation of this analysis is found in the $\mu-j-\nu$ event class. The selected $M_{\text {all }}$ region between $160 \mathrm{GeV}$ and $200 \mathrm{GeV}$ contains 3 data events for an expectation of $0.19 \pm 0.05$ events. In the chosen $\sum P_{T}$ region between $150 \mathrm{GeV}$ and $170 \mathrm{GeV}$ 3 data events are found while only $0.07 \pm 0.03$ events are predicted. About $3 \%(28 \%)$ of hypothetical HERA Monte Carlo experiments would produce a deviation more significant than the one observed in the corresponding sum of transverse momenta (invariant mass) distribution of this topology with a jet, a muon and a neutrino. 


\section{Appendix A}

\section{Resolutions}

\begin{tabular}{|c||c|c|c||c|c|c|}
\hline $\begin{array}{c}\text { event } \\
\text { class }\end{array}$ & $\begin{array}{c}\delta\left(M_{\text {all }}\right) \\
(\mathrm{GeV})\end{array}$ & $\begin{array}{c}\left\langle\delta\left(M_{\text {all }}\right)\right\rangle \\
(\mathrm{GeV})\end{array}$ & $\begin{array}{c}\left\langle\delta\left(M_{\text {all }}\right)\right\rangle_{w} \\
(\mathrm{GeV})\end{array}$ & $\begin{array}{c}\delta\left(\Sigma P_{T}\right) \\
(\mathrm{GeV})\end{array}$ & $\begin{array}{c}\left\langle\delta\left(\sum P_{T}\right)\right\rangle \\
(\mathrm{GeV})\end{array}$ & $\begin{array}{c}\left\langle\delta\left(\sum P_{T}\right)\right\rangle_{w} \\
(\mathrm{GeV})\end{array}$ \\
\hline$j-j$ & 4.3 & 9.8 & 5.2 & 3.9 & 9.4 & 4.5 \\
$e-j$ & 4.2 & 7.4 & 5.3 & 2.8 & 6.8 & 3.5 \\
$\mu-j$ & 3.7 & 11.5 & 6.9 & 3.0 & 16.2 & 5.7 \\
$j-\nu$ & 6.3 & 11.8 & 8.5 & 6.7 & 13.0 & 7.9 \\
$e-\nu$ & 9.1 & 10.6 & 7.1 & 3.0 & 2.7 & 3.6 \\
$e-e$ & 1.6 & 5.4 & 2.6 & 1.4 & 4.6 & 2.1 \\
$e-\mu$ & 1.9 & 10.6 & 4.6 & 1.7 & 10.1 & 4.2 \\
$\mu-\mu$ & 2.3 & 13.7 & 5.2 & 2.2 & 16.9 & 5.0 \\
$j-\gamma$ & 3.4 & 4.8 & 3.9 & 2.9 & 4.3 & 3.2 \\
$e-\gamma$ & 1.6 & 3.6 & 2.1 & 1.4 & 3.6 & 1.8 \\
$\gamma-\gamma$ & 1.6 & 3.6 & 2.1 & 1.4 & 3.6 & 1.8 \\
$j-j-j$ & 6.4 & 8.9 & 7.4 & 5.4 & 7.5 & 5.9 \\
$e-j-j$ & 5.7 & 7.4 & 6.8 & 4.5 & 6.7 & 5.3 \\
$j-j-\nu$ & 7.0 & 11.1 & 8.4 & 7.8 & 10.6 & 8.2 \\
$e-j-\nu$ & 9.3 & 13.9 & 14.4 & 5.2 & 8.1 & 7.5 \\
$\mu-j-\nu$ & 14.3 & 13.9 & 14.5 & 9.5 & 17.8 & 12.3 \\
$j-j-\gamma$ & 7.2 & 3.9 & 5.2 & 6.5 & 4.3 & 4.8 \\
$e-j-\gamma$ & 4.7 & 7.4 & 7.6 & 3.4 & 5.8 & 5.2 \\
$j-j-j-j$ & 6.0 & 7.0 & 6.8 & 4.8 & 7.2 & 5.8 \\
$e-j-j-j$ & 6.5 & 6.9 & 7.4 & 5.3 & 6.6 & 6.0 \\
$j-j-j-\nu$ & 7.6 & 6.9 & 7.1 & 8.6 & 8.3 & 7.7 \\
$e-j-j-j-j$ & 7.8 & 6.1 & 7.4 & 6.3 & 5.9 & 6.4 \\
\hline & & & & & & \\
\hline
\end{tabular}

Tab. A.1: The resolutions of $\sum P_{T}$ and $M_{\text {all }}$ for all event classes containing data events as derived from the investigation of the distributions displayed in figures A.1 - A.3. Since no sizeable SM expectation is available for the $\gamma-\gamma$ class, the resolutions determined from $e-\gamma$ events are adopted for this event class. While $\delta$ denotes the overall resolutions, their dependence on $\sum P_{T}$ and $M_{\text {all }}$ are considered by the arithmetic means $\langle\delta\rangle$ and event weighted means $\langle\delta\rangle_{w}$. 

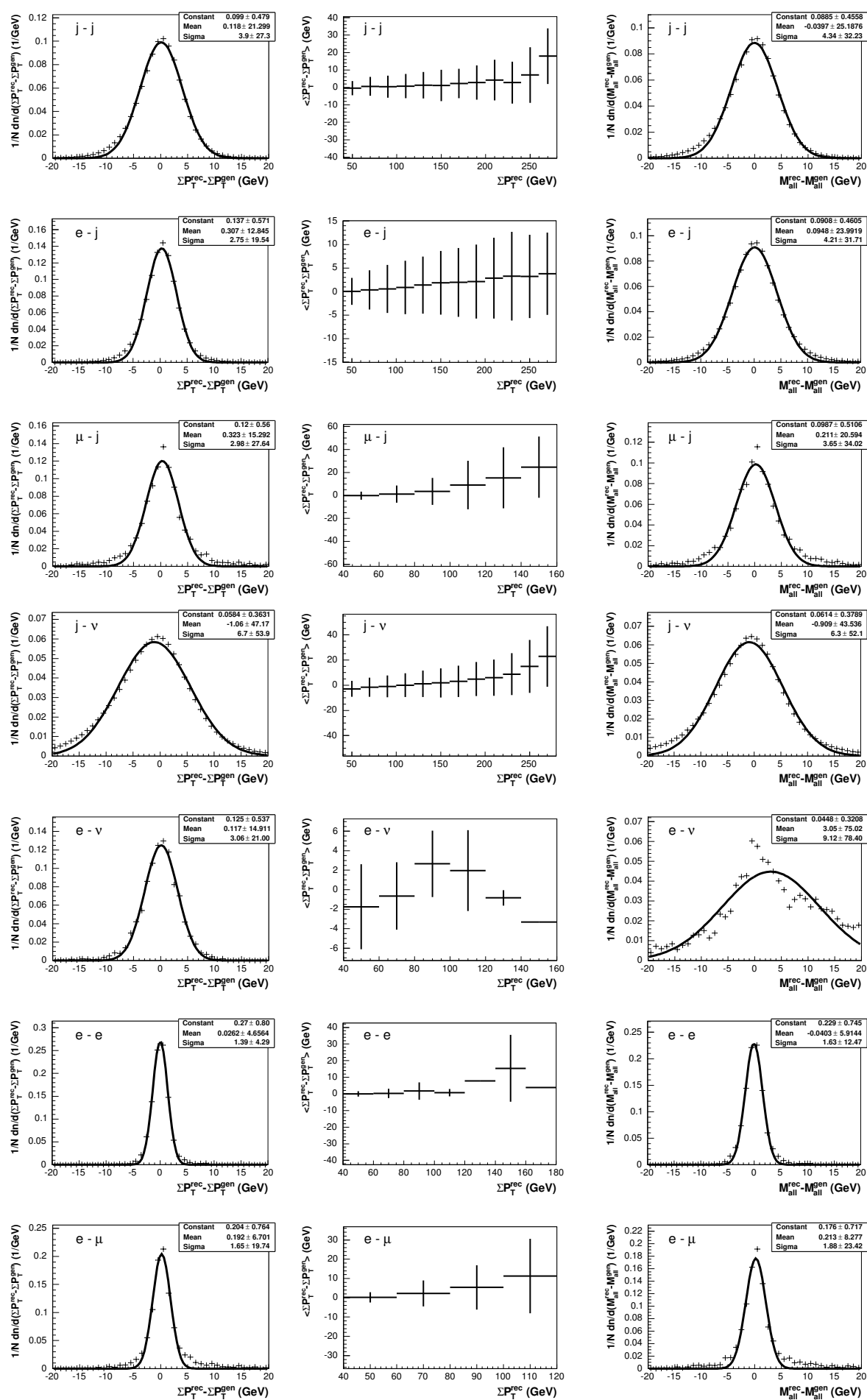
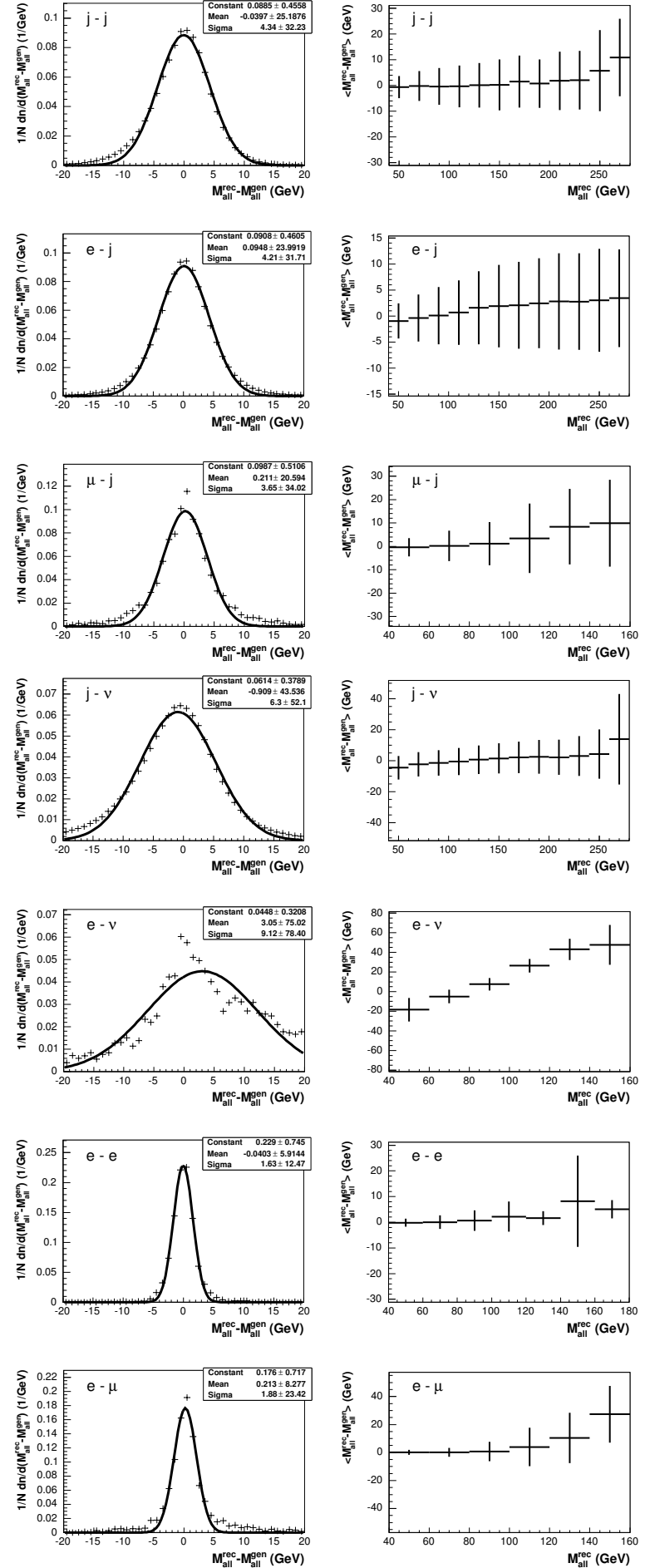

Fig. A.1: Resolutions of the sum of transverse momenta $\sum P_{T}$ (left side) and the invariant mass $M_{\text {all }}$ (right side) for event classes containing data events and the dependence of the resolutions on the reconstructed values of $\sum P_{T}$ and $M_{\text {all }}$, respectively. 

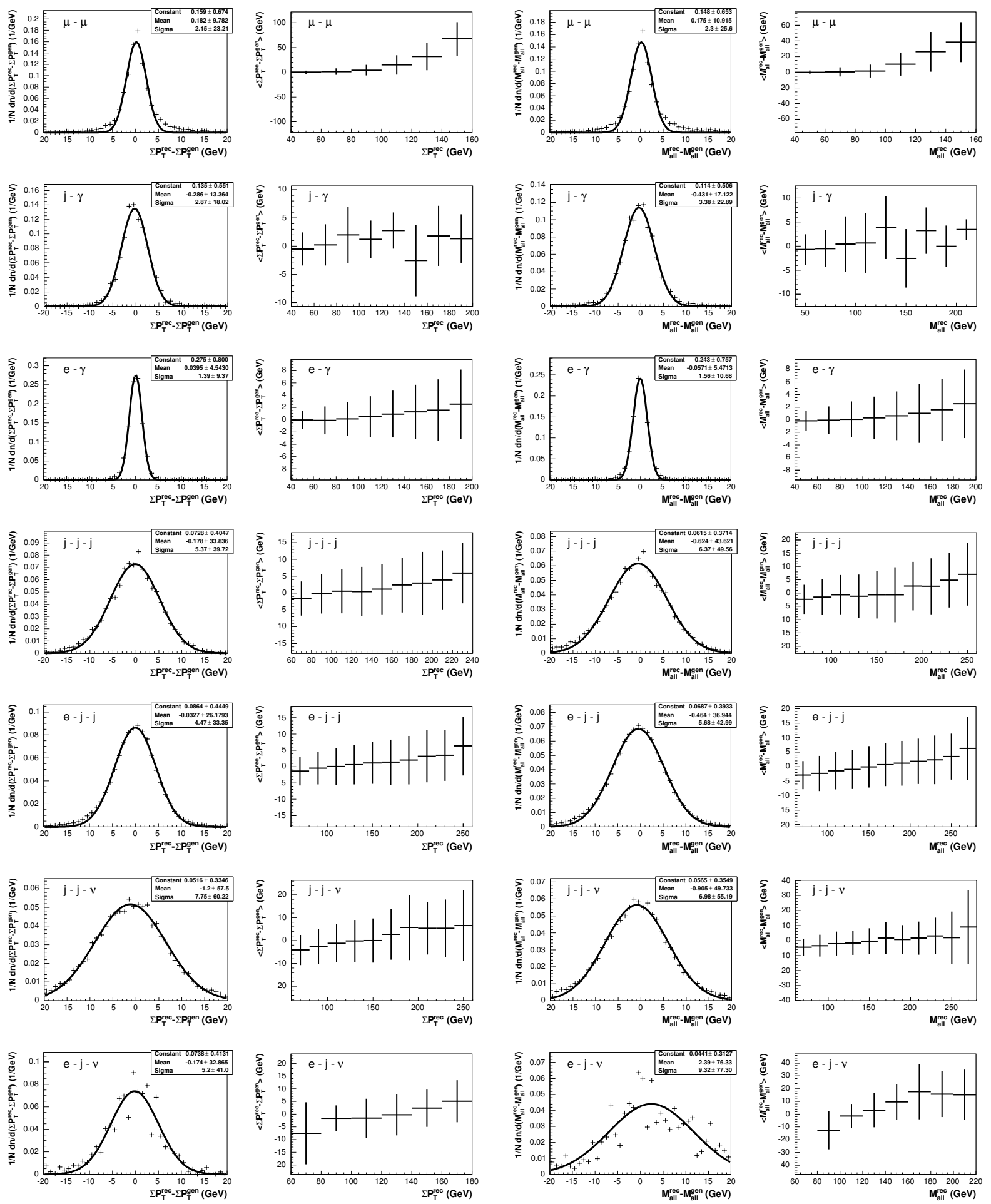

Fig. A.2: Resolutions of the sum of transverse momenta $\sum P_{T}$ (left side) and the invariant mass $M_{\text {all }}$ (right side) for event classes containing data events and the dependence of the resolutions on the reconstructed values of $\sum P_{T}$ and $M_{a l l}$, respectively. 

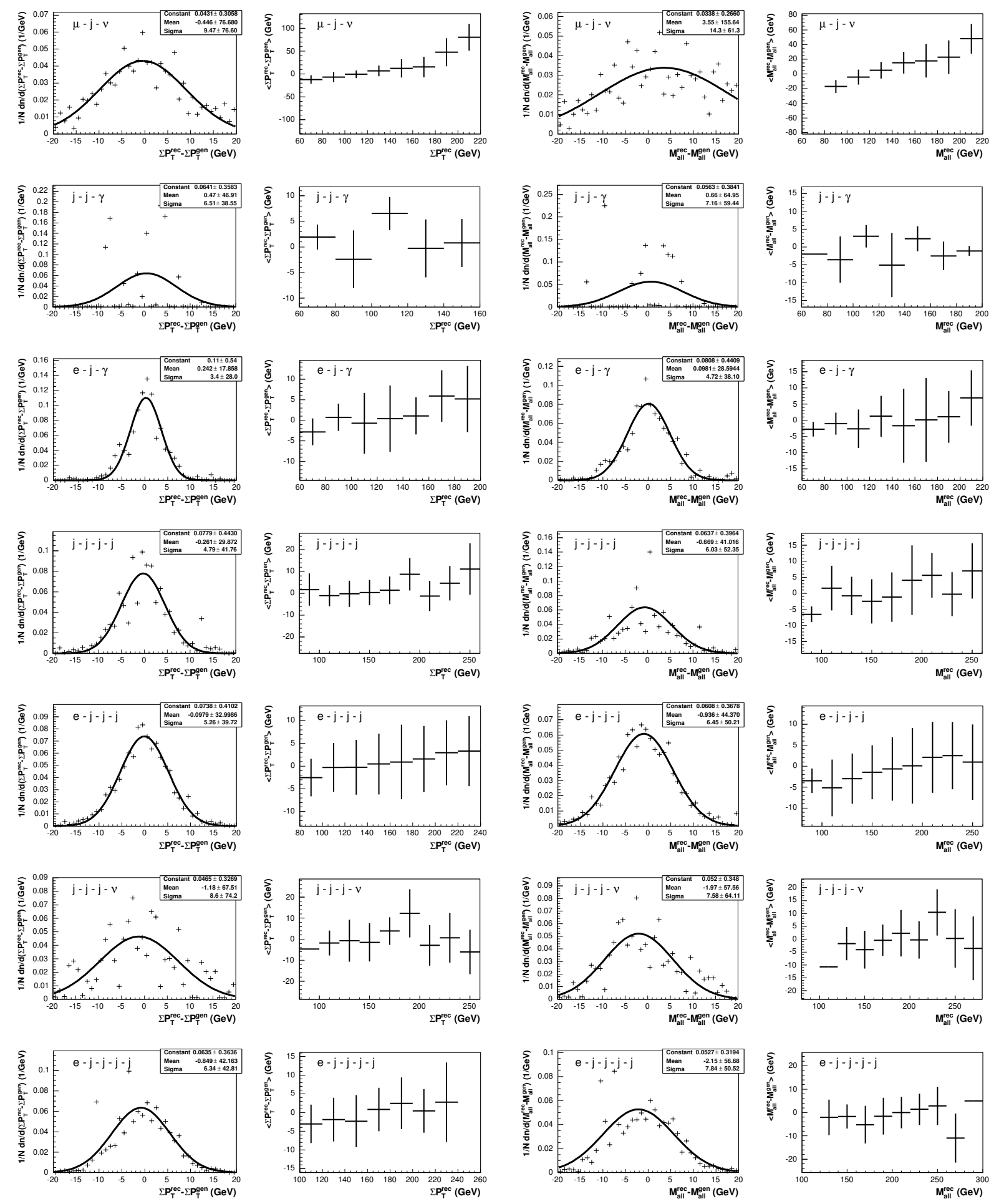

Fig. A.3: Resolutions of the sum of transverse momenta $\sum P_{T}$ (left side) and the invariant mass $M_{\text {all }}$ (right side) for event classes containing data events and the dependence of the resolutions on the reconstructed values of $\sum P_{T}$ and $M_{\text {all }}$, respectively. 


\section{Appendix B}

\section{Purities and Efficiencies}

\begin{tabular}{|c||c|c|c|c|}
\hline \multicolumn{1}{|c||}{} & \multicolumn{2}{|c|}{$\sum P_{T}$} & \multicolumn{2}{c|}{$M_{\text {all }}$} \\
\hline \hline event class & $\mathcal{P}(\%)$ & $\mathcal{E}(\%)$ & $\mathcal{P}(\%)$ & $\mathcal{E}(\%)$ \\
\hline$j-j$ & $80-95$ & $80-95$ & $75-90$ & $75-90$ \\
$e-j$ & $90-100$ & $60-75$ & $90-95$ & $60-70$ \\
$\mu-j$ & $70-85$ & $45-55$ & $75-80$ & $50-55$ \\
$j-\nu$ & $85-100$ & $80-90$ & $80-95$ & $75-90$ \\
$e-\nu$ & $30-70$ & $40-50$ & $20-75$ & $40-50$ \\
$e-e$ & $20-70$ & $20-40$ & $20-80$ & $25-40$ \\
$e-\mu$ & $80-95$ & $45-50$ & $80-90$ & $35-50$ \\
$\mu-\mu$ & $90-100$ & $20-30$ & $90-95$ & $20-30$ \\
$j-\gamma$ & $25-60$ & $5-15$ & $25-60$ & $5-15$ \\
$e-\gamma$ & $90-100$ & $40-55$ & $90-95$ & $40-55$ \\
$j-j-j$ & $60-85$ & $65-90$ & $60-85$ & $60-85$ \\
$e-j-j$ & $75-90$ & $45-60$ & $70-85$ & $45-60$ \\
$j-j-\nu$ & $60-85$ & $50-80$ & $55-75$ & $55-85$ \\
$e-j-\nu$ & $50-65$ & $35-45$ & $35-65$ & $35-50$ \\
$\mu-j-\nu$ & $55-65$ & $30-45$ & $50-65$ & $35-45$ \\
$j-j-\gamma$ & $5-15$ & $5-10$ & $10-15$ & $2-5$ \\
$e-j-\gamma$ & $75-85$ & $30-45$ & $75-85$ & $30-45$ \\
$j-j-j-j$ & $40-80$ & $40-90$ & $50-65$ & $50-85$ \\
$e-j-j-j$ & $50-80$ & $20-40$ & $40-70$ & $20-40$ \\
$j-j-j-\nu$ & $40-65$ & $35-60$ & $30-65$ & $30-60$ \\
$e-j-j-j-j$ & $30-65$ & $10-20$ & $40-60$ & $10-30$ \\
\hline
\end{tabular}

Tab. B.1: Typical values of purities $\mathcal{P}$ and efficiencies $\mathcal{E}$ as derived from the investigation of the distributions displayed in figures B.1 - B.3 for all event classes containing data events. 

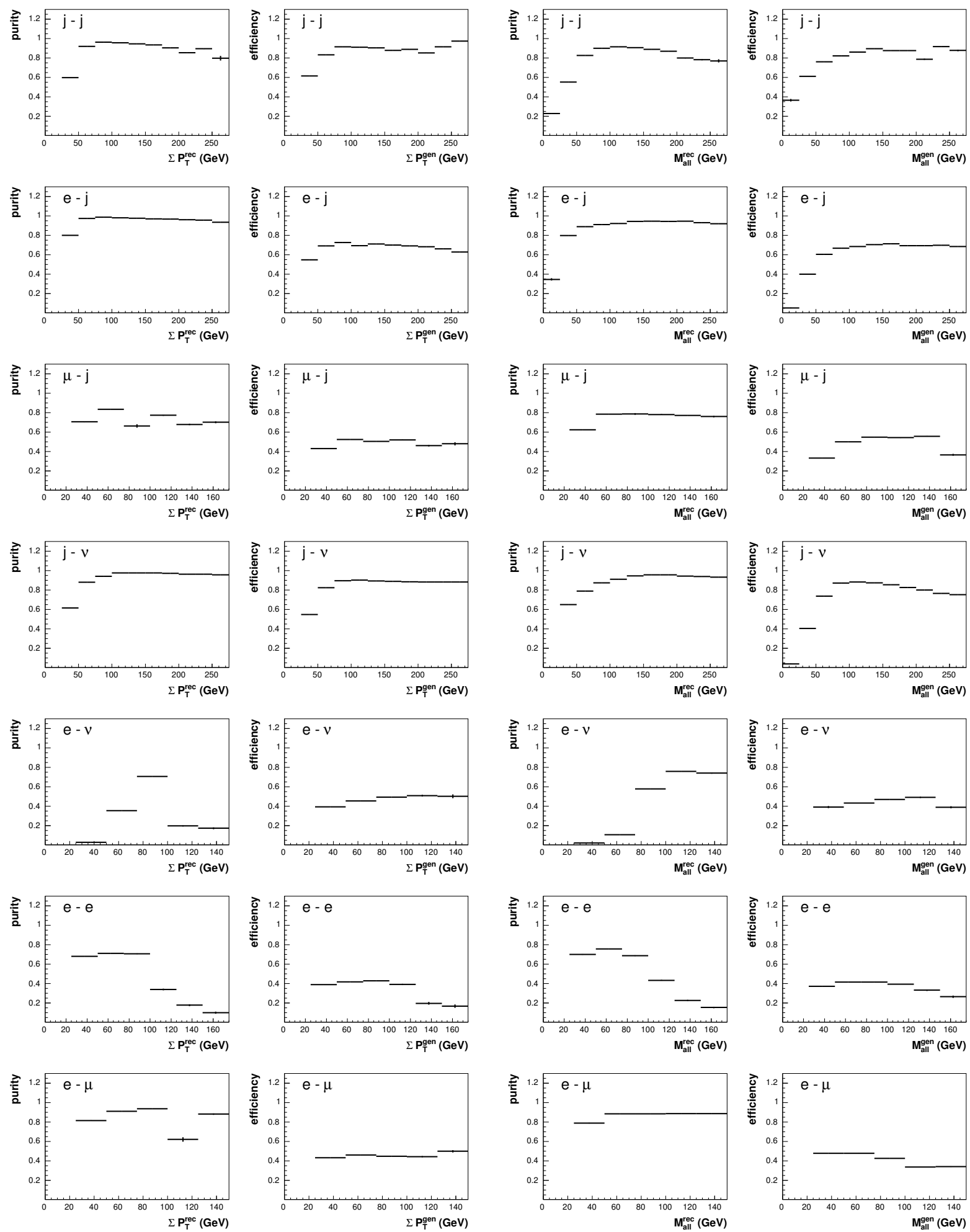

Fig. B.1: Purities and efficiencies as a function of the sum of transverse momenta $\sum P_{T}$ (left side) and the invariant mass $M_{\text {all }}$ (right side) for event classes containing data events. 

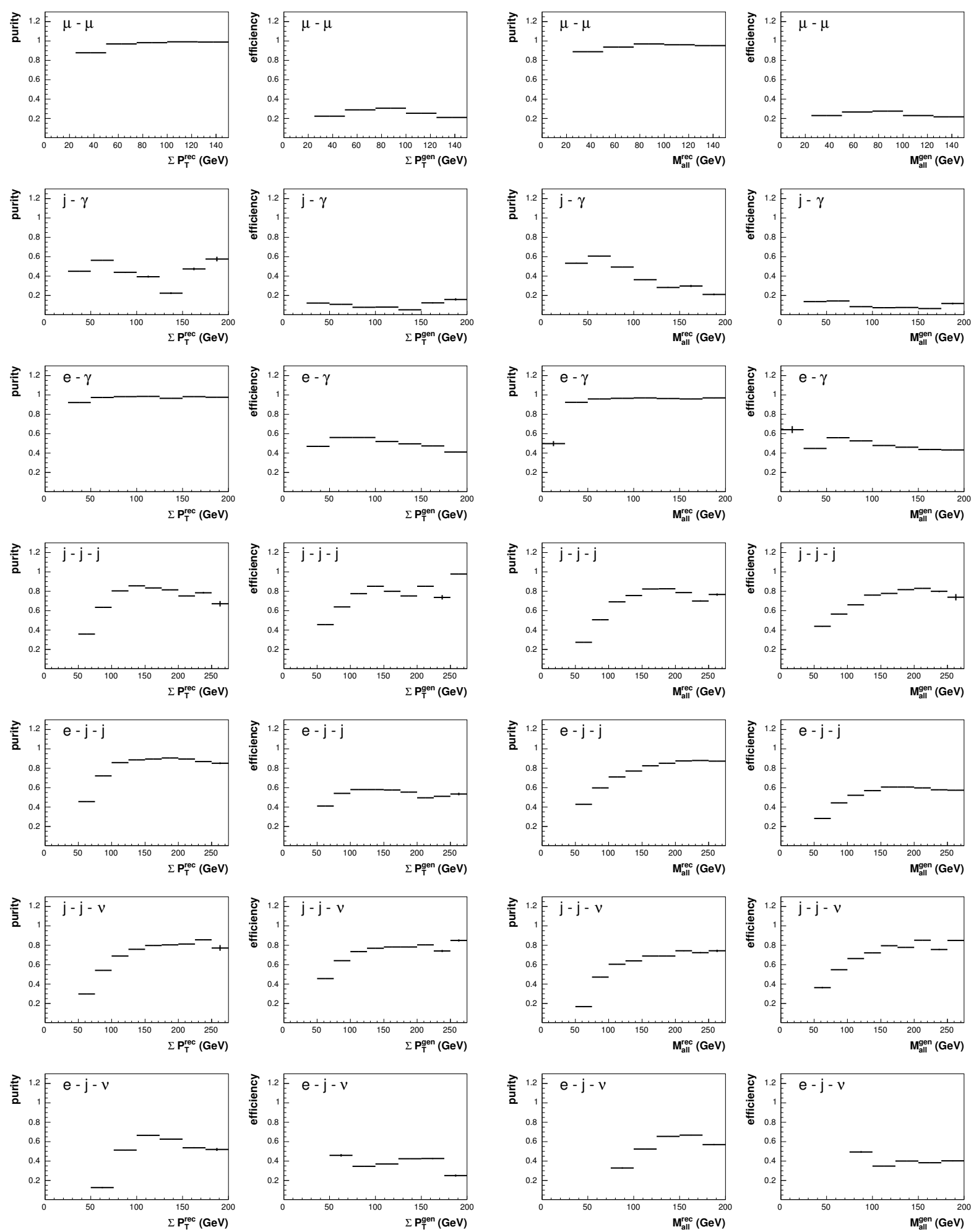

Fig. B.2: Purities and efficiencies as a function of the sum of transverse momenta $\sum P_{T}$ (left side) and the invariant mass $M_{\text {all }}$ (right side) for event classes containing data events. 

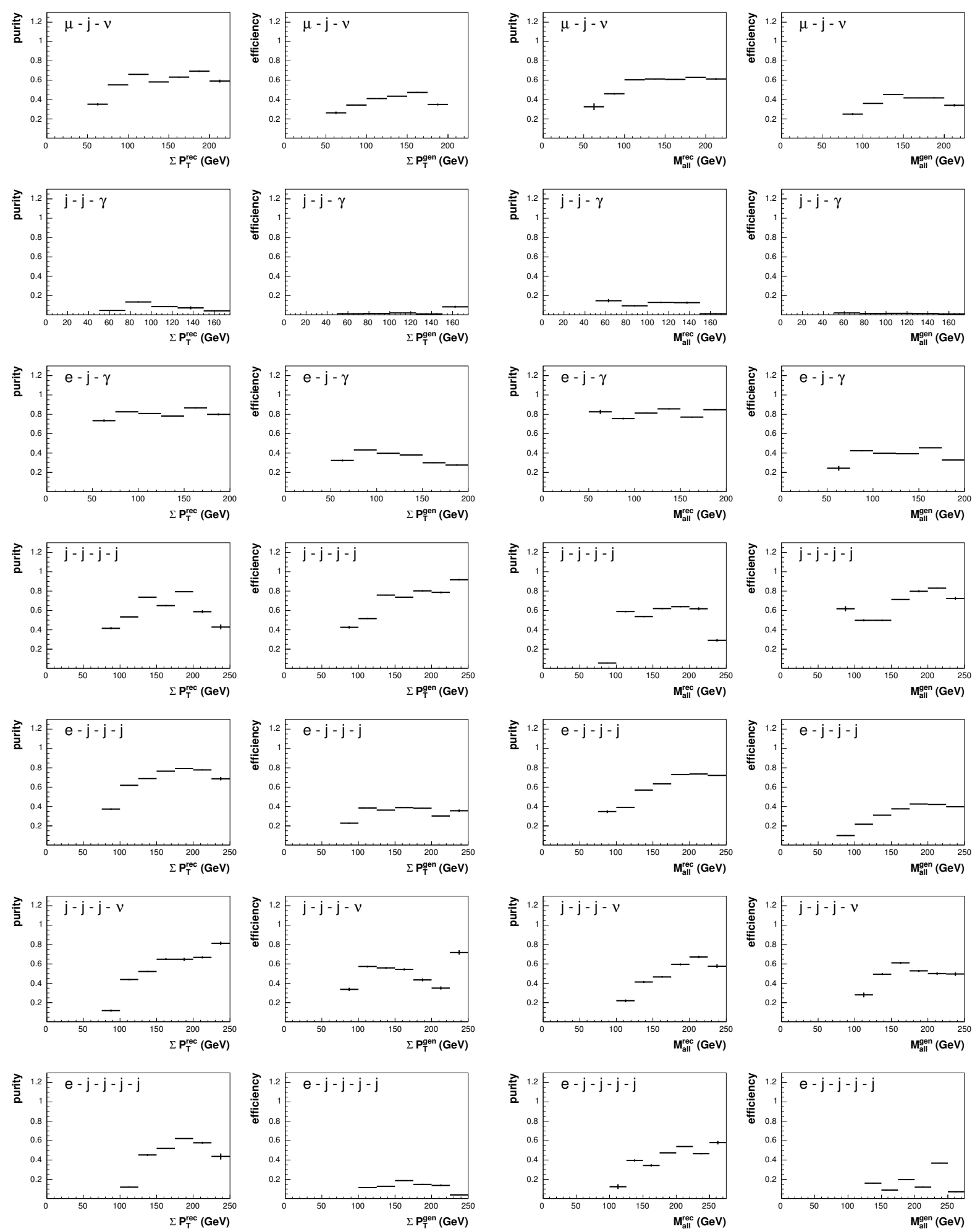

Fig. B.3: Purities and efficiencies as a function of the sum of transverse momenta $\sum P_{T}$ (left side) and the invariant mass $M_{\text {all }}$ (right side) for event classes containing data events. 


\section{Appendix C}

\section{Systematic Uncertainties}
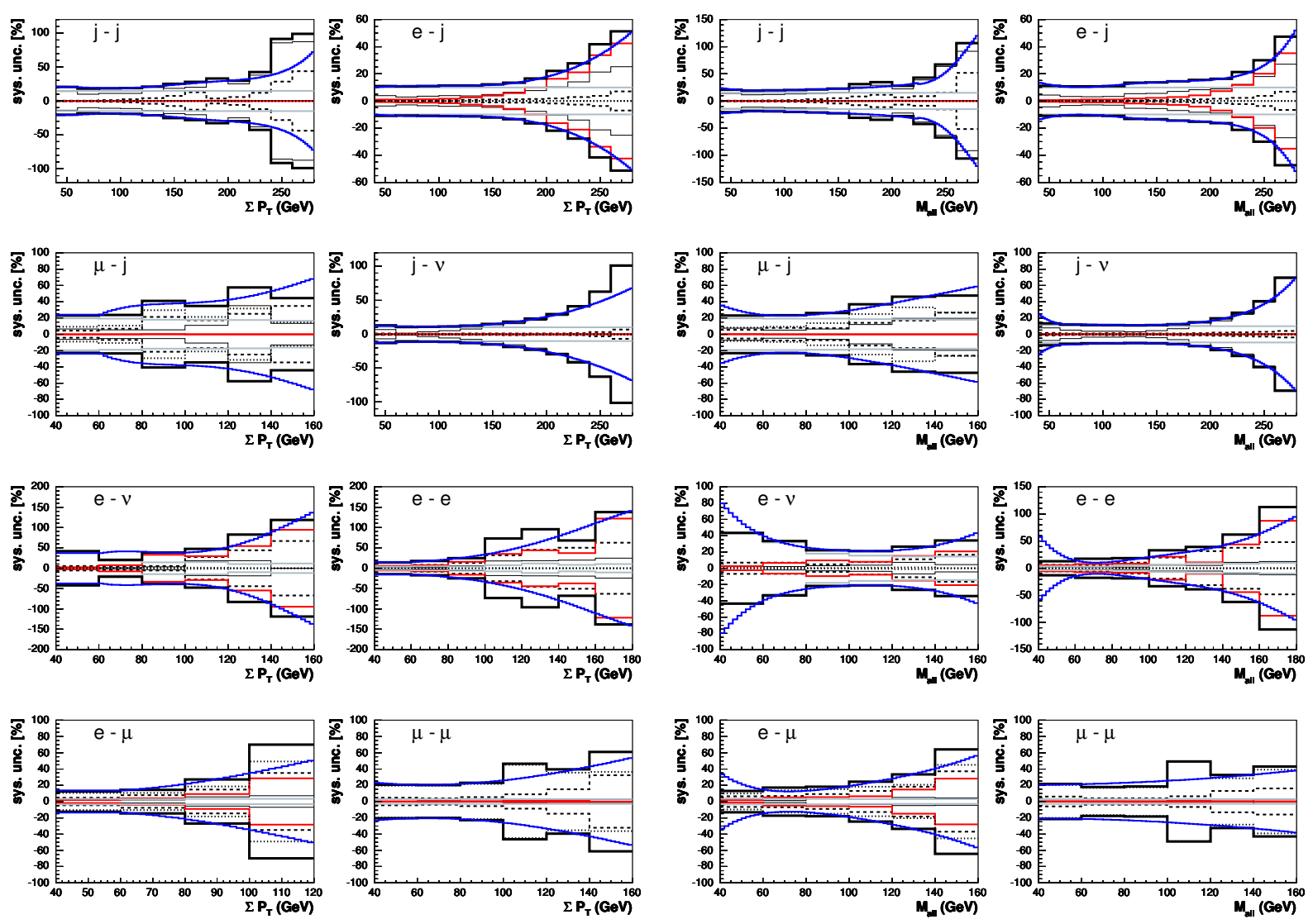

Fig. C.1: Systematic uncertainties on the predicted number of events as a function of $\sum P_{T}$ (left side) and $M_{\text {all }}$ (right side) for event classes containing data events. Only the total uncertainty and the corresponding fit as well as the uncertainties arising from the energy measurements and the Monte Carlo statistics are shown. 

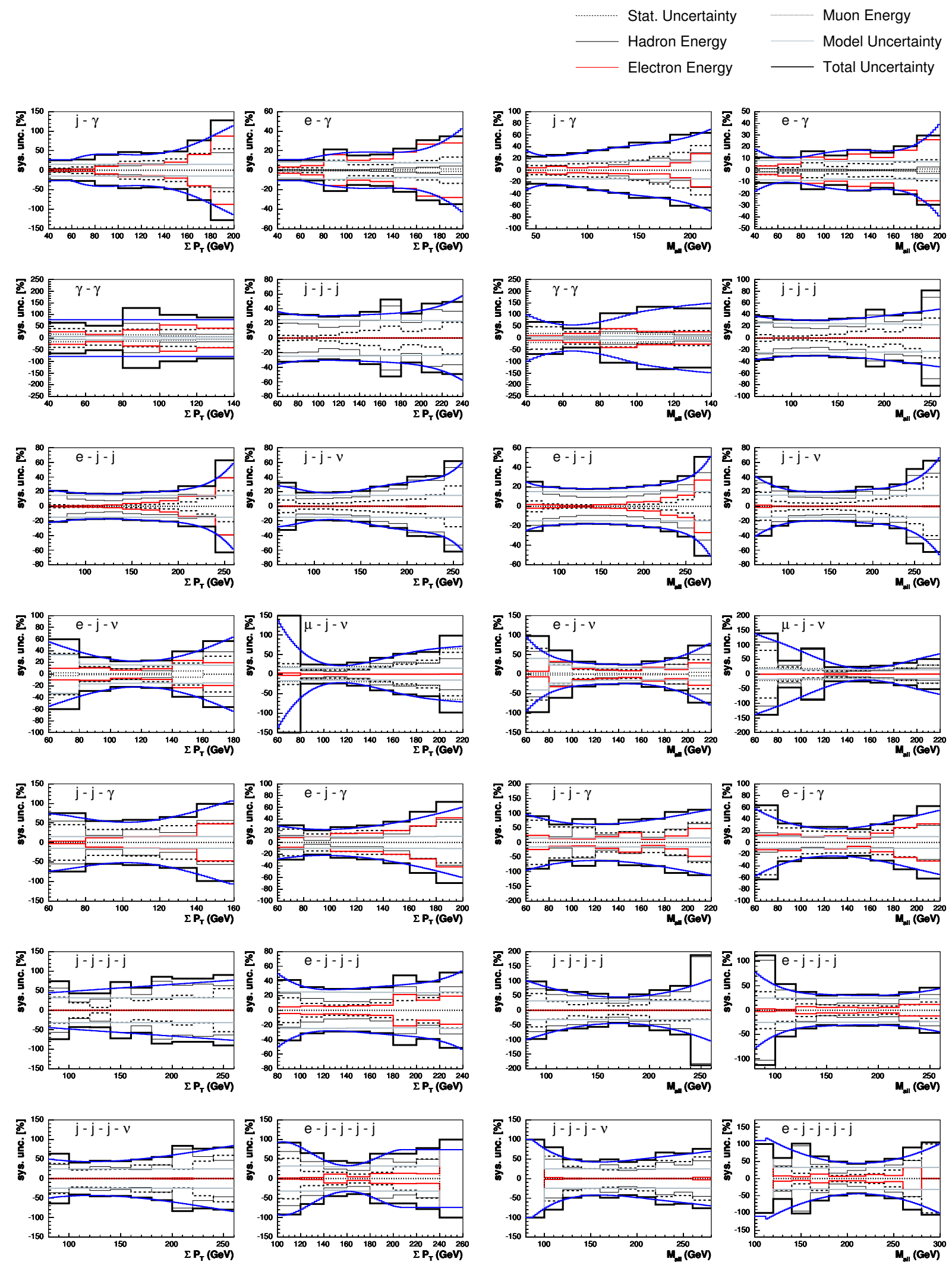

Fig. C.2: Systematic uncertainties on the predicted number of events as a function of $\sum P_{T}$ (left side) and $M_{\text {all }}$ (right side) for event classes containing data events. Only the total uncertainty and the corresponding fit as well as the uncertainties arising from the energy measurements and the Monte Carlo statistics are shown. 


\section{Appendix D}

\section{Event Display and Tables of the Results}
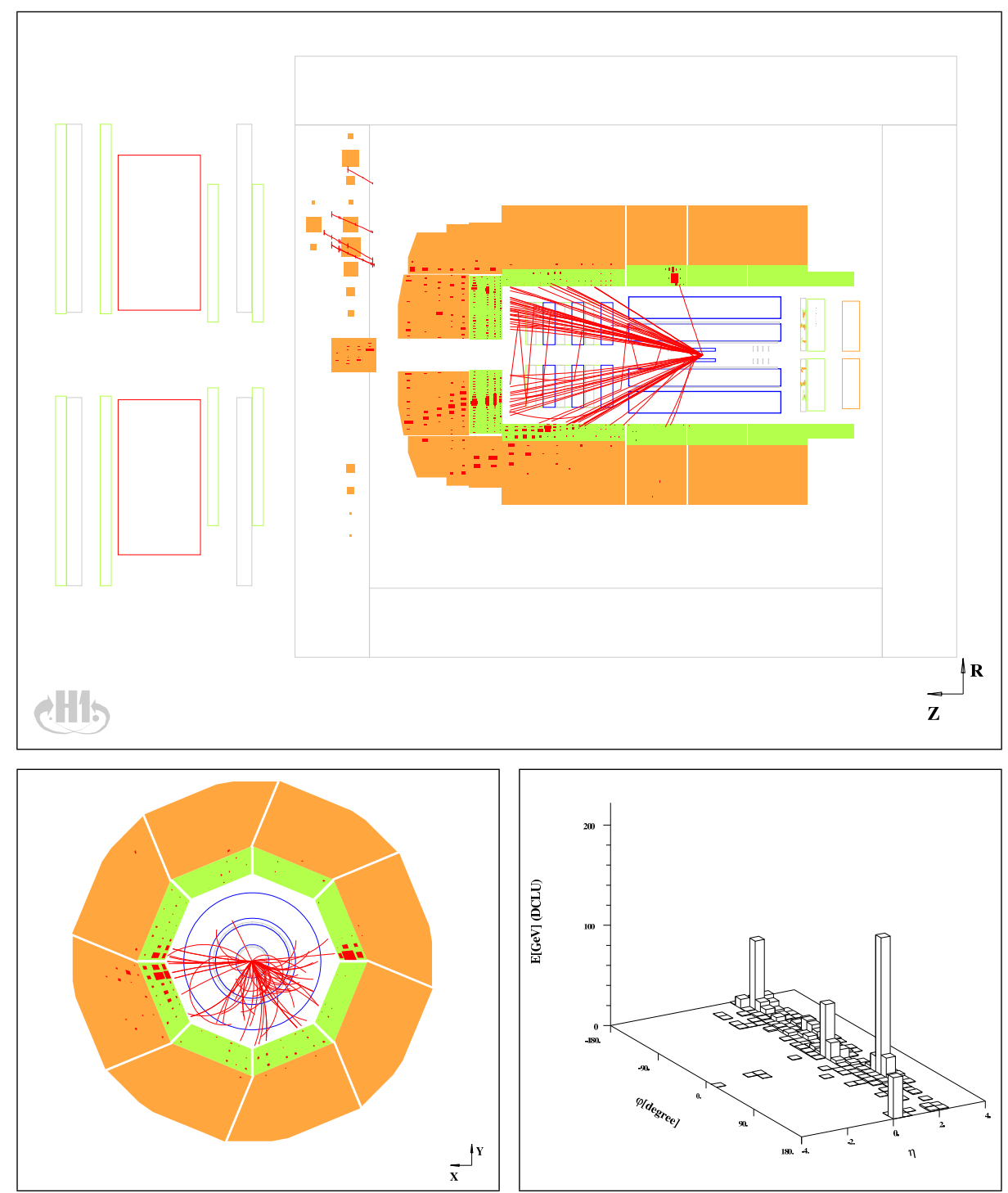

Fig. D.1: The event display of the $e-j-j-j-j$ event. Shown are the side view ( $r z$-plane), the radial view ( $x y$-plane) and the energy flow in the $\eta \phi$-plane. 


\begin{tabular}{|c|c|c|c|c|c|c|}
\hline event class & $N_{o b s}$ & $N_{S M}$ & \pm & $\delta N_{S M}^{t o t}$ & $\delta N_{S M}^{\text {syst }}$ & $\delta N_{S M}^{\text {stat }}$ \\
\hline$j-j$ & 31518 & 32439.6855 & \pm & 6578.4050 & 6578.2210 & 49.2019 \\
\hline$e-j$ & 30280 & 31820.9741 & \pm & 3483.6695 & 3483.5804 & 24.9098 \\
\hline$\mu-j$ & 5 & 5.4662 & \pm & 1.2358 & 1.2312 & 0.1070 \\
\hline$j-\nu$ & 2238 & 2215.5443 & \pm & 267.8261 & 267.7781 & 5.0702 \\
\hline$e-\nu$ & 9 & 12.8605 & \pm & 4.4587 & 4.4512 & 0.2580 \\
\hline$e-e$ & 8 & 11.1825 & \pm & 1.4358 & 1.4189 & 0.2193 \\
\hline$e-\mu$ & 4 & 4.8432 & \pm & 0.6062 & 0.5980 & 0.0995 \\
\hline$\mu-\mu$ & 6 & 2.7226 & \pm & 0.5515 & 0.5495 & 0.0473 \\
\hline$j-\gamma$ & 68 & 82.6451 & \pm & 21.4122 & 21.3377 & 1.7840 \\
\hline$e-\gamma$ & 158 & 170.4644 & \pm & 17.2223 & 17.0942 & 2.0967 \\
\hline$\mu-\gamma$ & 0 & 0.0439 & \pm & 0.0124 & 0.0098 & 0.0076 \\
\hline$\nu-\gamma$ & 0 & 2.0914 & \pm & 0.3270 & 0.3159 & 0.0846 \\
\hline$\gamma-\gamma$ & 1 & 1.1238 & \pm & 0.4976 & 0.4473 & 0.2180 \\
\hline$j-j-j$ & 454 & 476.4256 & \pm & 150.1452 & 150.0772 & 4.5186 \\
\hline$e-j-j$ & 328 & 354.1095 & \pm & 65.6511 & 65.6353 & 1.4388 \\
\hline$\mu-j-j$ & 0 & 0.0225 & \pm & 0.0162 & 0.0134 & 0.0091 \\
\hline$j-j-\nu$ & 46 & 47.9659 & \pm & 10.0502 & 10.0347 & 0.5583 \\
\hline$e-e-j$ & 0 & 0.1370 & \pm & 0.0386 & 0.0303 & 0.0239 \\
\hline$e-e-\nu$ & 0 & 0.0186 & \pm & 0.0084 & 0.0075 & 0.0037 \\
\hline$e-e-e$ & 0 & 0.0693 & \pm & 0.0356 & 0.0333 & 0.0126 \\
\hline$\mu-\mu-j$ & 0 & 0.0643 & \pm & 0.0185 & 0.0170 & 0.0074 \\
\hline$e-\mu-\mu$ & 0 & 0.0977 & \pm & 0.0170 & 0.0142 & 0.0094 \\
\hline$\mu-\mu-\nu$ & 0 & 0.0191 & \pm & 0.0097 & 0.0089 & 0.0037 \\
\hline$e-\mu-j$ & 0 & 0.0546 & \pm & 0.0396 & 0.0390 & 0.0067 \\
\hline$e-j-\nu$ & 2 & 0.8955 & \pm & 0.1681 & 0.1653 & 0.0305 \\
\hline$\mu-j-\nu$ & 4 & 0.7666 & \pm & 0.1856 & 0.1843 & 0.0218 \\
\hline$j-j-\gamma$ & 1 & 0.4296 & \pm & 0.1678 & 0.1591 & 0.0534 \\
\hline$e-j-\gamma$ & 2 & 6.1191 & \pm & 0.7487 & 0.7037 & 0.2556 \\
\hline$j-\nu-\gamma$ & 0 & 0.9778 & \pm & 0.1198 & 0.1092 & 0.0492 \\
\hline$e-j-j-j$ & 5 & 5.5942 & \pm & 1.6495 & 1.6433 & 0.1432 \\
\hline$j-j-j-\nu$ & 2 & 0.9583 & \pm & 0.2744 & 0.2689 & 0.0548 \\
\hline$j-j-\nu-\gamma$ & 0 & 0.0107 & \pm & 0.0061 & 0.0016 & 0.0059 \\
\hline$e-j-j-\gamma$ & 0 & 0.0162 & \pm & 0.0084 & 0.0029 & 0.0078 \\
\hline$e-e-j-\nu$ & 0 & 0.0136 & \pm & 0.0070 & 0.0063 & 0.0031 \\
\hline$e-\mu-j-\nu$ & 0 & 0.0113 & \pm & 0.0041 & 0.0032 & 0.0026 \\
\hline$j-j-j-j$ & 10 & 5.2245 & \pm & 2.2126 & 2.1848 & 0.3499 \\
\hline$e-j-j-j-j$ & 1 & 0.0261 & \pm & 0.0113 & 0.0111 & 0.0020 \\
\hline$j-j-j-j-\nu$ & 0 & 0.0168 & \pm & 0.0127 & 0.0102 & 0.0076 \\
\hline$j-j-j-j-j$ & 0 & 0.0180 & \pm & 0.0088 & 0.0080 & 0.0036 \\
\hline
\end{tabular}

Tab. D.1: The data and SM expectation for all event classes with a SM expectation greater than 0.01 events as derived from the investigation of the complete HERA-I data sample. 


\begin{tabular}{|c|c|c|c|c|c|c|c|c|c|c|c|}
\hline event class & $N_{o b s}$ & $N_{S M}$ & $\mathrm{NC}$ & $\mathrm{CC}$ & GP & $\mathrm{PG}$ & $\mathrm{EE}$ & MM & $\mathrm{TT}$ & QEDC & W \\
\hline$j-j$ & 31518 & 32439.686 & 4618.58 & 20.42 & 27753.42 & 2.03 & 0.93 & 0.77 & 0.58 & 0.00 & 42.95 \\
\hline$e-j$ & 30280 & 31820.974 & 31778.23 & 0.01 & 0.55 & 0.98 & 36.11 & 3.56 & 0.77 & 0.01 & 0.75 \\
\hline$\mu-j$ & 5 & 5.466 & 0.07 & 0.00 & 0.20 & 0.00 & 0.00 & 4.62 & 0.07 & 0.00 & 0.51 \\
\hline$j-\nu$ & 2238 & 2215.544 & 64.52 & 2027.09 & 116.46 & 0.62 & 0.09 & 1.36 & 0.93 & 0.00 & 4.46 \\
\hline$e-\nu$ & 9 & 12.860 & 7.25 & 0.16 & 0.00 & 0.00 & 0.43 & 0.56 & 0.50 & 0.08 & 3.88 \\
\hline$e-e$ & 8 & 11.182 & 1.04 & 0.00 & 0.00 & 0.00 & 9.52 & 0.01 & 0.04 & 0.55 & 0.02 \\
\hline$e-\mu$ & 4 & 4.843 & 0.19 & 0.00 & 0.00 & 0.00 & 0.00 & 4.57 & 0.06 & 0.00 & 0.02 \\
\hline$\mu-\mu$ & 6 & 2.723 & 0.00 & 0.00 & 0.00 & 0.00 & 0.00 & 2.72 & 0.00 & 0.00 & 0.00 \\
\hline$j-\gamma$ & 68 & 82.645 & 60.37 & 0.45 & 3.94 & 17.78 & 0.05 & 0.00 & 0.01 & 0.01 & 0.02 \\
\hline$e-\gamma$ & 158 & 170.464 & 55.08 & 0.00 & 0.00 & 0.00 & 0.09 & 0.04 & 0.03 & 115.23 & 0.00 \\
\hline$\mu-\gamma$ & 0 & 0.044 & 0.00 & 0.00 & 0.00 & 0.00 & 0.00 & 0.04 & 0.00 & 0.00 & 0.00 \\
\hline$\nu-\gamma$ & 0 & 2.091 & 0.11 & 1.86 & 0.00 & 0.01 & 0.00 & 0.01 & 0.01 & 0.10 & 0.00 \\
\hline$\gamma-\gamma$ & 1 & 1.124 & 0.13 & 0.00 & 0.00 & 0.00 & 0.00 & 0.00 & 0.00 & 0.99 & 0.00 \\
\hline$j-j-j$ & 454 & 476.426 & 83.30 & 0.87 & 385.83 & 0.02 & 0.00 & 0.01 & 0.01 & 0.00 & 6.39 \\
\hline$e-j-j$ & 328 & 354.110 & 353.47 & 0.00 & 0.00 & 0.00 & 0.10 & 0.02 & 0.01 & 0.00 & 0.50 \\
\hline$\mu-j-j$ & 0 & 0.023 & 0.00 & 0.00 & 0.01 & 0.00 & 0.00 & 0.01 & 0.00 & 0.00 & 0.00 \\
\hline$j-j-\nu$ & 46 & 47.966 & 1.61 & 38.68 & 7.40 & 0.00 & 0.00 & 0.02 & 0.02 & 0.00 & 0.24 \\
\hline$e-e-j$ & 0 & 0.137 & 0.05 & 0.00 & 0.00 & 0.00 & 0.06 & 0.00 & 0.00 & 0.00 & 0.03 \\
\hline$e-e-\nu$ & 0 & 0.019 & 0.00 & 0.00 & 0.00 & 0.00 & 0.00 & 0.00 & 0.00 & 0.00 & 0.02 \\
\hline$e-e-e$ & 0 & 0.069 & 0.00 & 0.00 & 0.00 & 0.00 & 0.07 & 0.00 & 0.00 & 0.00 & 0.00 \\
\hline$\mu-\mu-j$ & 0 & 0.064 & 0.00 & 0.00 & 0.00 & 0.00 & 0.00 & 0.06 & 0.00 & 0.00 & 0.00 \\
\hline$e-\mu-\mu$ & 0 & 0.098 & 0.00 & 0.00 & 0.00 & 0.00 & 0.00 & 0.10 & 0.00 & 0.00 & 0.00 \\
\hline$\mu-\mu-\nu$ & 0 & 0.019 & 0.00 & 0.00 & 0.00 & 0.00 & 0.00 & 0.02 & 0.00 & 0.00 & 0.00 \\
\hline$e-\mu-j$ & 0 & 0.055 & 0.00 & 0.00 & 0.00 & 0.00 & 0.00 & 0.02 & 0.00 & 0.00 & 0.03 \\
\hline$e-j-\nu$ & 2 & 0.895 & 0.07 & 0.02 & 0.00 & 0.00 & 0.00 & 0.01 & 0.01 & 0.00 & 0.78 \\
\hline$\mu-j-\nu$ & 4 & 0.767 & 0.00 & 0.00 & 0.00 & 0.00 & 0.00 & 0.06 & 0.00 & 0.00 & 0.71 \\
\hline$e-\mu-\nu$ & 0 & 0.039 & 0.00 & 0.00 & 0.00 & 0.00 & 0.00 & 0.03 & 0.00 & 0.00 & 0.01 \\
\hline$j-j-\gamma$ & 1 & 0.430 & 0.28 & 0.01 & 0.09 & 0.04 & 0.00 & 0.00 & 0.00 & 0.00 & 0.01 \\
\hline$e-j-\gamma$ & 2 & 6.119 & 6.11 & 0.00 & 0.00 & 0.00 & 0.01 & 0.00 & 0.00 & 0.00 & 0.00 \\
\hline$j-\nu-\gamma$ & 0 & 0.978 & 0.00 & 0.97 & 0.00 & 0.00 & 0.00 & 0.00 & 0.00 & 0.00 & 0.00 \\
\hline$e-j-j-j$ & 5 & 5.594 & 5.44 & 0.00 & 0.00 & 0.00 & 0.00 & 0.00 & 0.00 & 0.00 & 0.16 \\
\hline$j-j-j-\nu$ & 2 & 0.958 & 0.01 & 0.74 & 0.19 & 0.00 & 0.00 & 0.00 & 0.00 & 0.00 & 0.02 \\
\hline$j-j-\nu-\gamma$ & 0 & 0.011 & 0.00 & 0.01 & 0.00 & 0.00 & 0.00 & 0.00 & 0.00 & 0.00 & 0.00 \\
\hline$e-j-j-\gamma$ & 0 & 0.016 & 0.02 & 0.00 & 0.00 & 0.00 & 0.00 & 0.00 & 0.00 & 0.00 & 0.00 \\
\hline$e-e-j-\nu$ & 0 & 0.014 & 0.00 & 0.00 & 0.00 & 0.00 & 0.00 & 0.00 & 0.00 & 0.00 & 0.01 \\
\hline$e-\mu-j-\nu$ & 0 & 0.011 & 0.00 & 0.00 & 0.00 & 0.00 & 0.00 & 0.00 & 0.00 & 0.00 & 0.01 \\
\hline$j-j-j-j$ & 10 & 5.224 & 0.77 & 0.04 & 4.42 & 0.00 & 0.00 & 0.00 & 0.00 & 0.00 & 0.00 \\
\hline$e-j-j-j-j$ & 1 & 0.026 & 0.03 & 0.00 & 0.00 & 0.00 & 0.00 & 0.00 & 0.00 & 0.00 & 0.00 \\
\hline$j-j-j-j-\nu$ & 0 & 0.017 & 0.00 & 0.02 & 0.00 & 0.00 & 0.00 & 0.00 & 0.00 & 0.00 & 0.00 \\
\hline$j-j-j-j-j$ & 0 & 0.018 & 0.00 & 0.00 & 0.02 & 0.00 & 0.00 & 0.00 & 0.00 & 0.00 & 0.00 \\
\hline
\end{tabular}

Tab. D.2: The data and SM expectation for all event classes with a SM expectation greater than 0.01 events as derived from the investigation of the complete HERA-I data sample. In addition the individual contributions to the SM expectation arising from the different physical processes are listed (NC: neutral current DIS, CC: charged current DIS, GP: photoproduction, PG: prompt photon production, EE: electron pair production, MM: muon pair production, TT: tau pair production, QEDC: QED Compton scattering, $\mathrm{W}: W$ production). 


\section{List of Figures}

1.1 Diagrams for electron-proton scattering . . . . . . . . . . . 6

1.2 Diagrams for jet production . . . . . . . . . . . . . . . . 14

1.3 Diagrams for resolved jet production . . . . . . . . . . . . 15

1.4 Diagrams for prompt photon production . . . . . . . . . . . 16

1.5 Diagrams for real photon radiation . . . . . . . . . . . . 16

1.6 Diagrams for real photon radiation . . . . . . . . . . . . . . . . . . 17

1.7 Diagrams for lepton pair production . . . . . . . . . . . . . . 18

1.8 Diagrams for real $W$ production . . . . . . . . . . . . . . . . 20

2.1 Electron proton storage ring HERA . . . . . . . . . . . . . . . . . . . . . . . . . 22

2.2 Integrated luminosity delivered by HERA . . . . . . . . . . . . . . . 23

2.3 H1 detector at HERA . . . . . . . . . . . . . . . . . . . . 25

2.4 Side view of the inner tracking system . . . . . . . . . . . . 27

2.5 Isometric view of the CIP detector . . . . . . . . . . . . . . . 28

2.6 Side view of the LAr calorimeter . . . . . . . . . . . . . 30

2.7 Module of the Central Muon Detector . . . . . . . . . . . . . . . . . . 32

2.8 Luminosity system of the $\mathrm{H} 1$ detector . . . . . . . . . . . . . . . 33

4.1 Event yields as a function of the integrated luminosity . . . . . . . . . . 44

4.2 Distributions of $E_{R=0.75} / E^{e}$ and $E_{R=1.00} / E^{e}$ for the inclusive electron sample 47

4.3 Distributions of $D C A_{c l}^{t r}$ and $D_{t r}^{t r}$ for the inclusive electron sample . . . . . 48

4.4 Distributions of $\Delta z_{C I P}$ and $R_{\text {start }}$ for the inclusive electron sample . . . . 50

4.5 Distributions of $P_{T}$-match for the inclusive electron sample . . . . . . . . 50

4.6 Distributions of $P_{T}$ and $\theta$ for the inclusive electron sample . . . . . . . . 51

4.7 Electron track association efficiency depending on the electron track criteria 53

4.8 Electron track association efficiency as a function of $P_{T}$ and $\theta \quad \ldots . . .54$

4.9 Distributions of the quantities used for photon identification . . . . . . . 55

4.10 Distributions of $P_{T}$ and $\theta$ for the inclusive photon sample . . . . . . . 57

4.11 CIP inefficiency as a function of $P_{T}$ and $\theta \ldots \ldots \ldots$. . . . . . . . 58

4.12 Electron misidentification probability as a function of $P_{T}$ and $\theta \quad \ldots . .59$

4.13 Purity and efficiency of the $j-j$ class before and after application of the jet identification criteria . . . . . . . . . . . . . . . 62

4.14 Distributions of $\sum P_{T}$ for the $e-j, j-\nu$ and $j-j$ event classes $\ldots \ldots . \ldots 6$ 
5.1 Electromagnetic energy scale uncertainty . . . . . . . . . . . . . 68

5.2 Hadronic energy scale uncertainty determined by $P_{T}^{\text {jet }} / P_{T}^{d a} \ldots \ldots$. . . . 71

5.3 Hadronic energy scale uncertainty determined by $P_{T}^{\text {had }} / P_{T}^{\text {da }} \ldots \ldots \ldots$. . . . 72

5.4 Distributions of $P_{T}$ for an low- $P_{T}$ electron sample . . . . . . . . . 76

5.5 Distributions of $P_{T}^{e}$ and $P_{T}$ for the $e-\nu$ event class. . . . . . . . . . 76

5.6 Distributions of $P_{T}, \phi$ and $\theta$ for the $\mu-\nu$ event class $\ldots \ldots \ldots$. . . . . 78

5.7 Distribution of $P_{T}^{\text {jet }}$ for the $\mu-\nu$ event class $\ldots \ldots \ldots \ldots$. . . . . . . 79

5.8 Resolutions of $\sum P_{T}$ for the $j-j, e-j, \mu-j$ and $j-\nu$ event classes $\ldots \ldots$. . . 81

5.9 Purities and efficiencies for various event classes . . . . . . . . . . . . 83

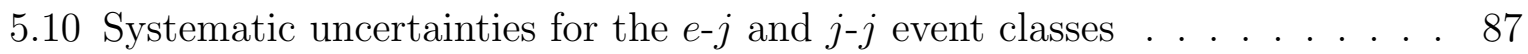

5.11 Distributions of $M_{\text {all }}$ and $\sum P_{T}$ for the low- $P_{T} j-j-j-j$ event sample . . . 88

5.12 Distributions of $P_{T}$ and $\theta$ for jets in the low- $P_{T} e-j-j-j-j$ event sample . . . 90

5.13 Distributions of $M_{\text {all }}$ and $\sum P_{T}$ for the low- $P_{T} e-j-j-j-j$ event sample . . 90

6.1 Event yields of all event classes for the HERA-I data sample . . . . . . . . 92

6.2 Distributions of $M_{\text {all }}$ for the $e-j, j-\nu$ and $j-j$ event classes . . . . . . . . . 93

6.3 Distributions of $M_{\text {all }}$ for the $e-e, \mu-\mu, e-\mu$ and $\mu-j$ event classes . . . . . . 94

6.4 Distributions of $\sum P_{T}$ and $M_{\text {all }}$ for the $\mu-j-\nu$ event class . . . . . . . . . 95

6.5 Distributions of $\sum P_{T}$ for the $e-j-j, j-j-\nu, j-j-j, e-j-j-j, j-j-j-\nu$ event classes 96

6.6 Distributions of $\sum P_{T}$ and $M_{\text {all }}$ for the $e-j-j-j-j$ event class . . . . . . . 97

6.7 Event yields of all event classes for the $e^{+} p$ and $e^{-} p$ data samples . . . . . 98

6.8 Event yields of all event classes for the low- $P_{T}$ and high- $P_{T}$ event samples . 100

$7.1 M_{\text {all }}$ regions of greatest deviation for the $e-j, j-\nu$ and $j-j$ event classes . . 103

7.2 Distributions of $-\log _{10} \hat{P}$ for the $e-j, j-\nu$ and $j-j$ event classes . . . . . . 106

7.3 Sensitivity on anomalous top production . . . . . . . . . . . . 108

7.4 Sensitivity on leptoquark production . . . . . . . . . . . . . . . 109

7.5 Sensitivity on $R$-parity violating stop production . . . . . . . . . . . . . 110

7.6 Distributions of $M_{\text {all }}$ and $\sum P_{T}$ including various signals of new physics . . 111

7.7 Distributions of $-\log _{10} \hat{P}$ for the HERA-I data sample . . . . . . . . . 115

7.8 Distributions of $-\log _{10} \hat{P}$ for the low- $P_{T}$ and high- $P_{T}$ event samples . . . . 116

7.9 Distributions of $M_{\text {all }}$ for all event classes containing data events . . . . . . 118

7.10 Distributions of $\sum P_{T}$ for all event classes containing data events . . . . . 119

A.1 Distributions of the resolutions of $\sum P_{T}$ and $M_{\text {all }} \ldots \ldots \ldots \ldots$

A.2 Distributions of the resolutions of $\sum P_{T}$ and $M_{\text {all }} \ldots \ldots \ldots \ldots$

A.3 Distributions of the resolutions of $\sum P_{T}$ and $M_{\text {all }} \ldots \ldots \ldots \ldots$

B.1 Purities and efficiencies as a function of $\sum P_{T}$ and $M_{\text {all }} \ldots \ldots . \ldots 128$

B.2 Purities and efficiencies as a function of $\sum P_{T}$ and $M_{\text {all }} \ldots \ldots$. . . . . . 129

B.3 Purities and efficiencies as a function of $\sum P_{T}$ and $M_{\text {all }} \ldots \ldots$. . . . 130

C.1 Systematic uncertainties as a function of $\sum P_{T}$ and $M_{\text {all }} \ldots \ldots \ldots 131$ 
C.2 Systematic uncertainties as a function of $\sum P_{T}$ and $M_{\text {all }} \ldots \ldots \ldots$. . . 132

D.1 Event display of the $e-j-j-j-j$ event . . . . . . . . . . . 133 


\section{List of Tables}

3.1 Monte Carlo samples . . . . . . . . . . . . . . . . . . . 40

4.1 HERA-I data samples . . . . . . . . . . . . . . . . . . . . 42

4.2 Basic event selection criteria . . . . . . . . . . . . . . . 43

4.3 Electron estimators used by QECFWD . . . . . . . . . . . . . 46

4.4 Electron identification criteria $\ldots \ldots \ldots \ldots \ldots \ldots \ldots$

4.5 Photon identification criteria . . . . . . . . . . . . . . 56

4.6 Muon identification criteria . . . . . . . . . . . . . . 60

4.7 Jet identification criteria . . . . . . . . . . . . . . . . . 61

4.8 Neutrino identification criteria . . . . . . . . . . . . . . 63

5.1 Selection criteria for the neutral current DIS event sample used for the verification of the hadronic energy calibration . . . . . . . . . 70

5.2 The muon, jet and $\not_{T}$ trigger and their consideration in this analysis $\ldots 74$

5.3 Systematic uncertainties attributed to the particle measurements . . . . . 8 85

5.4 Theoretical uncertainties attributed to different SM processes . . . . . . . 86

7.1 Minimal region sizes considered by the search algorithm . . . . . . . . . 102

7.2 Various values for the likeliness $P_{X}^{n} \ldots \ldots \ldots \ldots \ldots \ldots \ldots$

7.3 Values of $\hat{P}, p, N_{o b s}$ and $N_{S M}$ as derived by the search algorithm . . . 113

A.1 Resolutions of $\sum P_{T}$ and $M_{\text {all }} \ldots \ldots \ldots \ldots \ldots \ldots \ldots \ldots \ldots \ldots$

B.1 Typical values of purities and efficiencies . . . . . . . . . . 127

D.1 Number of data events and the corresponding SM expectation . . . . . 134

D.2 Individual contributions to the SM expectation . . . . . . . . 135 


\section{Bibliography}

[1] M. Kuze and Y. Sirois, Prog. Part. Nucl. Phys. 50, 1 (2003), hep-ex/0211048.

[2] M. Wessels, General Search for New Phenomena in ep Scattering at HERA, contribution to the proceedings of the International Europhysics Conference on High Energy Physics, Aachen, Germany, 2003.

[3] H1, General Search for New Phenomena in ep Scattering at HERA, submitted to the XXI International Symposium on Lepton and Photon Interactions at High Energies, Batavia, Illinois, USA, 2003.

[4] H. Abramowicz and A. Caldwell, Rev. Mod. Phys. 71, 1275 (1999), hep-ex/9903037.

[5] CTEQ, R. Brock et al., Rev. Mod. Phys. 67, 157 (1995).

[6] C. Berger, Elementarteilchenphysik (Springer-Verlag, 2002).

[7] D. Griffiths, Elementarteilchenphysik (Akademia Verlag, 1996).

[8] F. Halzen and A. Martin, Quarks And Leptons (John Wiley \& Sons, 1984).

[9] D. Perkins, Introduction to High Energy Physics (Cambridge University Press, 2000).

[10] H1, C. Adloff et al., Eur. Phys. J. C30, 1 (2003), hep-ex/0304003.

[11] C. F. von Weizsacker, Z. Phys. 88, 612 (1934).

[12] E. J. Williams, Phys. Rev. 45, 729 (1934).

[13] A. Arbuzov, D. Y. Bardin, J. Bluemlein, L. Kalinovskaya, and T. Riemann, Comput. Phys. Commun. 94, 128 (1996), hep-ph/9511434.

[14] N. Arteaga-Romero, C. Carimalo, and P. Kessler, Z. Phys. C52, 289 (1991).

[15] U. Baur, J. A. M. Vermaseren, and D. Zeppenfeld, Nucl. Phys. B375, 3 (1992).

[16] H1, I. Abt et al., Nucl. Instrum. Meth. A386, 310 (1997).

[17] S. Bentvelsen, J. Engelen, and P. Kooijman, Proceedings, Physics at HERA, vol. 1, DESY, Hamburg, Germany (1991). 
[18] F. Jacquet and A. Blondel, Proceedings, Study of an ep Facility for Europe 97/48, ed. U. Amaldi, DESY, Hamburg, Germany (1979).

[19] H1, C. Adloff et al., Eur. Phys. J. C25, 13 (2002), hep-ex/0201006.

[20] T. Sjostrand et al., Comput. Phys. Commun. 135, 238 (2001), hep-ph/0010017.

[21] B. Andersson, G. Gustafson, G. Ingelman, and T. Sjostrand, Phys. Rept. 97, 31 (1983).

[22] M. Gluck, E. Reya, and A. Vogt, Phys. Rev. D45, 3986 (1992).

[23] CTEQ, H. L. Lai et al., Eur. Phys. J. C12, 375 (2000), hep-ph/9903282.

[24] H. Jung, Comp. Phys. Commun. 86, 147 (1995).

[25] A. Kwiatkowski, H. Spiesberger, and H. J. Mohring, Comp. Phys. Commun. 69, 155 (1992).

[26] G. A. Schuler and H. Spiesberger, In *Hamburg 1991, Proceedings, Physics at HERA, vol. 3* 1419-1432. (see HIGH ENERGY PHYSICS INDEX 30 (1992) No. 12988).

[27] L. Lonnblad, Comput. Phys. Commun. 71, 15 (1992).

[28] C. Berger and P. Kandel, Prepared for Workshop on Monte Carlo Generators for HERA Physics (Plenary Starting Meeting), Hamburg, Germany, 27-30 Apr 1998.

[29] T. Abe, Comput. Phys. Commun. 136, 126 (2001), hep-ph/0012029.

[30] A. Mucke, R. Engel, J. P. Rachen, R. J. Protheroe, and T. Stanev, Comput. Phys. Commun. 124, 290 (2000), astro-ph/9903478.

[31] K.-P. O. Diener, C. Schwanenberger, and M. Spira, Eur. Phys. J. C25, 405 (2002), hep-ph/0203269.

[32] K.-P. O. Diener, C. Schwanenberger, and M. Spira, (2003), hep-ex/0302040.

[33] P. Aurenche, P. Chiappetta, M. Fontannaz, J. P. Guillet, and E. Pilon, Z. Phys. C56, 589 (1992).

[34] H. L. Lai et al., Phys. Rev. D55, 1280 (1997), hep-ph/9606399.

[35] R. Brun, F. Bruyant, M. Maire, A. C. McPherson, and P. Zanarini, CERN$\mathrm{DD} / \mathrm{EE} / 84-1$.

[36] G. Grindhammer, M. Rudowicz, and S. Peters, Nucl. Instrum. Meth. A290, 469 (1990).

[37] H1, V. Andreev et al., Phys. Lett. B561, 241 (2003), hep-ex/0301030. 
[38] H1, A. Aktas et al., Eur. Phys. J. C31, 17 (2003), hep-ex/0307015.

[39] E. Chabert et al., QBGFMAR: An Updated Phan Package for Cosmic and Halo Muon Topological Rejection in High $P_{T}$ Physics Analysis, 1998, H1 internal note 556.

[40] B. Leissner, Muon-Pair Production in Electron-Proton Collisions, Phd thesis, RWTH-Aachen, Germany, 2002, DESY-THESIS-2002-049.

[41] G. Frising, Rare Phenomena and W Production in Electron Proton Scattering at HERA, Phd thesis, RWTH-Aachen, Germany, 2003,

http://www-h1.desy.de/publications/theses_list.html.

[42] H1, A. Aktas et al., (2003), hep-ex/0311015.

[43] P. Bruel, Recherche d'interactions au-dela du Modele Standard a HERA, Phd thesis, Univ. Paris XI, France, 1998.

[44] U. Bassler et al., QESCAT: Electron Identification Software in H1PHAN, H1 Software Package.

[45] L. West, How to use the Heavy Flavour Working Group Track, Muon and Electron Selection Code, 2000, H1 Software Package.

[46] V. Blobel, HAMBURG DESY - INTERNAL REP. R1-88-01 (88,REC.FEB.) 40p.

[47] M. Stoye, Untersuchung von Endzuständen mit mehreren Elektronen in der ElektronProton Streuung, Diploma thesis, RWTH-Aachen, Germany, 2003, http://mozart.physik.rwth-aachen.de/Diplom-Doktor.html.

[48] S. D. Ellis and D. E. Soper, Phys. Rev. D48, 3160 (1993), hep-ph/9305266.

[49] S. Catani, Y. L. Dokshitzer, M. H. Seymour, and B. R. Webber, Nucl. Phys. B406, 187 (1993).

[50] ZEUS, J. Breitweg et al., Phys. Rev. D63, 052002 (2001), hep-ex/0009059.

[51] H1, C. Adloff et al., Eur. Phys. J. C13, 609 (2000), hep-ex/9908059.

[52] A. Vest, Search for Bosonic Stop Decays in R-Parity Violating Supersymmetry in $e+q$ Collisions at HERA, Phd thesis, RWTH-Aachen, Germany, 2003, http://www-h1.desy.de/publications/theses_list.html.

[53] H1, C. Schwanenberger, (2002), physics/0209026, published in *Pasadena 2002, Calorimetry in particle physics* $761-766$.

[54] H1, I. Abt et al., Nucl. Instrum. Meth. A386, 348 (1997). 
[55] S. Caron, Jets in Photoproduction at HERA, Phd thesis, RWTH-Aachen, Germany, 2002, DESY-THESIS-2002-035.

[56] M. Peez, Recherche de deviations au Model Standard dans les processus de grande energie transverse sur le collisionneur electron-proton HERA, Phd thesis, Univ. Lyon, France, 2003, DESY-THESIS-2003-023.

[57] D. M. South, Events with Isolated Leptons and Missing Transverse Momentum in $e+p$ Collisions at HERA, Phd thesis, Univ. of Manchester, Great Britain, 2003, DESY-THESIS-2003-030.

[58] H1, C. Adloff et al., Phys. Lett. B523, 234 (2001), hep-ex/0107038.

[59] H1, C. Adloff et al., Eur. Phys. J. C19, 289 (2001), hep-ex/0010054.

[60] H1, C. Adloff et al., Phys. Lett. B515, 17 (2001), hep-ex/0106078.

[61] M. Wobisch, Measurement and QCD Analysis of Jet Cross Sections in Deep-Inelastic Positron-Proton Collisions at sqrt(s)=300 GeV, Phd thesis, RWTH-Aachen, Germany, 1999, DESY-THESIS-2000-049, PITHA 00/12.

[62] D0, V. M. Abazov et al., Phys. Rev. D67, 052001 (2003), hep-ex/0207046.

[63] OPAL, M. Z. Akrawy et al., Phys. Lett. B235, 389 (1990).

[64] L3, B. Adeva et al., Phys. Lett. B248, 227 (1990).

[65] D0, B. Abbott et al., Phys. Rev. D62, 092004 (2000), hep-ex/0006011.

[66] D0, B. Abbott et al., Phys. Rev. D64, 012004 (2001), hep-ex/0011067.

[67] H1, A. Aktas et al., Eur. Phys. J. C33, 9 (2004), hep-ex/0310032.

[68] W. Buchmuller, R. Ruckl, and D. Wyler, Phys. Lett. B191, 442 (1987).

[69] H1, C. Adloff et al., Eur. Phys. J. C11, 447 (1999), hep-ex/9907002.

[70] ZEUS, S. Chekanov et al., Phys. Rev. D68, 052004 (2003), hep-ex/0304008.

[71] D. I. Kazakov, (2000), hep-ph/0012288. 


\section{Acknowledgement}

In the first place I want to thank my supervisor Prof. Dr. Christoph Berger for his guidance, support and advice. Moreover I want to thank him for the opportunity to accomplish my Diploma and PhD thesis directly at the DESY site, experiencing authentic experimental particles physics. I am grateful to Prof. Dr. Wolfgang Braunschweig for agreeing to be my second referee.

Since this complex and extensive analysis would have never been managed single-handedly I would like to thank especially Dr. Sascha Caron and Dr. Gilles Frising for the harmonic collaboration during the last three years. For the useful and instructive discussions contributing to the success of this analysis I am thankful to Dr. Hans-Ulrich Martyn, Dr. Matti Peez and Dr. Emmanuel Sauvan.

To all my room mates during my time in 1d33 I am grateful for their support and friendship. Thanks a lot Dr. Carlo Duprel, Lars Finke, Dr. Thomas Kluge, Linus Lindfeld, Dr. Peer-Oliver Meyer, Dr. Markus Wobisch and Anja Vest. In the same way I wish to thank my former colleagues from the Aachen group, Adil Aktas, Dr. Thomas Hadig, Dr. Boris Leissner, Jan Olzem, Carsten Schmitz and Dr. Jürgen Scheins. Special thanks to all of them who participate in proofreading of this thesis.

I owe special thanks to Kerstin for all her support and sympathy and for a beautiful time. Finally, I want to thank the loving care of my family, without it, this work would never have been possible. 



\title{
Lebenslauf von
}

\author{
Martin Wessels \\ Diplom Physiker
}

geboren am 20.09.1973 in Hannover

Familienstand: ledig

Staatsangehörigkeit: deutsch

08/1979 - 01/1983 Wilhelm-Hauff-Grundschule in Neu-Isenburg

02/1983 - 07/1983 Grundschule Burgholzhausen in Friedrichsdorf i.Ts.

08/1983 - 06/1992 Gymnasium Kaiserin-Friedrich-Schule in Bad Homburg v.d.H.

Abschluss: Abitur

07/1992 - 09/1993 Zivildienst in Bad Homburg v.d.H.

10/1993 - 03/1994 Studium des Bauingenieurwesens an der RWTH Aachen

04/1994 - 03/1999 Studium der Physik an der RWTH Aachen

04/1999 - 08/2000 Erstellung der Diplomarbeit am I. Physikalischen Institut B der RWTH Aachen bei Prof. Dr. Ch. Berger im Rahmen der H1-Kollaboration am DESY in Hamburg

Titel der Arbeit: Untersuchungen zur Elektron-Proton-Streuung mit Hilfe des VLQ-Spektrometers

$09 / 2000$

Diplom in Physik

03/2001 - 05/2004 Erstellung der Dissertation am I. Physikalischen Institut B der RWTH Aachen bei Prof. Dr. Ch. Berger im Rahmen der H1-Kollaboration am DESY in Hamburg

Titel der Arbeit: General Search For New Phenomena in $e p$ Scattering at HERA

29. Juli 2004

Mündliche Prüfung und Abschluss der Promotion 